\title{
Die Stabilisierung biegedrillknickgefährdeter Träger durch Sandwichelemente und Trapezbleche
}

\author{
Zur Erlangung des akademischen Grades eines \\ DOKTOR-INGENIEURS \\ von der Fakultät für \\ Bauingenieur-, Geo- und Umweltwissenschaften \\ der Universität Fridericiana zu Karlsruhe (TH) \\ genehmigte \\ DISSERTATION \\ von \\ Dipl.-Ing. Markus Dürr \\ aus Pforzheim
}

Tag der mündlichen Prüfung: 09.01.2008

Hauptreferent:

Prof. Dr.-Ing. Helmut Saal

Korreferent:

Prof. Dr.-Ing. Werner Wagner

Karlsruhe 2008 


\section{Vorwort}

Die vorliegende Arbeit entstand während meiner Tätigkeit als wissenschaftlicher Angestellter an der Versuchsanstalt für Stahl, Holz und Steine der Universität Karlsruhe $(\mathrm{TH})$.

Mein ganz besonderer Dank gilt Herrn Prof. Dr.-Ing. H. Saal für die Anregung und die kompetente Betreuung der Dissertation sowie die gute Zusammenarbeit in zahlreichen Industrie- und Forschungsprojekten. Seine unermüdliche Diskussionsbereitschaft und die darüber hinausgehende persönliche Unterstützung haben wesentlich zum Gelingen der Arbeit beigetragen.

Großer Dank gilt auch Herrn Prof. Dr.-Ing. W. Wagner für seine fachlichen Anregungen und die Übernahme des Korreferats.

Dank gilt ebenso allen Kollegen an der Versuchsanstalt für Stahl, Holz und Steine und am Institut für Stahl- und Leichtmetallbau der Universität Karlsruhe (TH) für die stets vorhandene Hilfsbereitschaft und das persönliche Miteinander.

Dem Arbeitskreis Technische Grundlagen des Industrieverbandes für Bausysteme im Metallleichtbau (IFBS) danke ich für das rege Interesse an meiner Arbeit und die konstruktiven Anregungen aus zahlreichen Diskussionen. Für die finanzielle Unterstützung der Versuche danke ich dem Industrieverband für Bausysteme im Metallleichtbau (IFBS) und dem Deutschen Institut für Bautechnik (DIBt).

Schließlich gebührt mein besonderer Dank meiner Frau Corinna für das Verständnis, die stetige Unterstützung und den starken Rückhalt während der Erstellung dieser Arbeit sowie unseren beiden Kindern Linus und Lucie, die mit ihrem wertvollen Lachen und ihrer Unbekümmertheit für den notwendigen Ausgleich zum wissenschaftlichen Alltag sorgten. Ohne sie wäre diese Arbeit so nicht möglich gewesen. 


\section{Kurzfassung}

In dieser Arbeit wird die stabilisierende Wirkung von Sandwichelementen und Trapezprofilen auf biegedrillknickgefährdete Träger untersucht. Dabei wird zum einen die Schubsteifigkeit von zweiseitig gelagerten Stahltrapezprofilen bezüglich der Einschränkung der seitlichen Ausweichmöglichkeit, zum anderen die drehbettende Wirkung von Sandwichelementen in Bezug auf die Behinderung der Verdrehungen des stabilitätsgefährdeten Trägers untersucht. Für beide Stabilisierungsarten werden mit Hilfe von experimentellen Untersuchungen und numerischen Berechnungen Regeln entwickelt, die den Ansatz der stabilisierenden Wirkung bei der Bemessung ermöglichen.

Die statistische Auswertung experimenteller Ergebnisse und begleitende parametrische Untersuchungen mit einem verifizierten Finite-Element-Modell zeigen, dass die drehstabilisierende Wirkung von Sandwichelementen mit Kernschichten aus Polyurethan-Hartschaum oder Mineralfasern durch eine trilineare MomentenVerdrehungsbeziehung erfasst werden kann. Für unterschiedliche Profiltypen und Sandwichelemente in allen üblichen Ausführungen und Anwendungsbereichen werden einfache Formeln angegeben, mit denen die zur Darstellung der MomentenVerdrehungsbeziehung notwendigen Parameter ermittelt werden können. Aus dieser Beziehung kann die Anschlusssteifigkeit in Abhängigkeit der Auflast der Träger als Sekantensteifigkeit ermittelt werden und mit ihr die für den Nachweis der Biegedrillknicksicherheit benötigte Drehfedersteifigkeit bestimmt werden.

Die Untersuchung der stabilisierenden Wirkung von zweiseitig gelagerten Schubfeldern aus Stahltrapezprofilen erfolgt auf Grundlage parametrischer Berechnungen mit einem Finite-Element-Modell, das zuvor durch Vergleich mit analytischen Werten und experimentellen Untersuchungen verifiziert wurde. Die numerischen Berechnungen berücksichtigen die Nachgiebigkeiten der Befestigungsmittel am Querrand sowie die Anzahl und Ausbildung der Längsstoßverbindungen. Die zur Nutzung der stabilisierenden Wirkung notwendige Schubsteifigkeit bei zweiseitiger Lagerung ergibt sich auf Grundlage der numerischen Untersuchungen in Abhängigkeit der bekannten Schubsteifigkeit bei allseitiger Lagerung durch Berücksichtigung mehrerer Abminderungsfaktoren. 


\section{Abstract}

Adjacent members like trapezoidal sheeting or sandwich panels can reduce the problem of lateral torsional buckling of beams by providing stabilization either by shear stiffness or by torsional restraint. Both types are investigated in this thesis by tests and numerical calculations with finite elements. This concerns the shear stiffness of steel sheeting diaphragms with only two edges fastened against lateral displacements of the compression chord of the beam and the torsional restraint of the rotation of the beam by sandwich panels.

The statistical evaluation of experimental investigations and parametric analyses by means of a verified finite element model show that the torsional restraint of sandwich panels with polyurethane or mineral wool core material can be expressed by a trilinear moment-rotation relationship. Formulae are given for different profile types combined with sandwich panels for all common constructions and fields of application to calculate the parameters of this relationship. The rotational stiffness of the connection which governs the torsional restraint behaviour can be taken from this relationship as secant stiffness depending on the lateral load of the beam.

The basis for the investigation of profiled steel sheeting diaphragms with fastening along two edges are numerical calculations with a finite element model which was verified by comparison with analytical calculations as well as with full-scale diaphragm tests. The flexibility of the fasteners which connect the sheeting to the supporting structure as well as the number and type of the seam fasteners are included in the numerical calculations. The result of the investigation are knock-down-factors which allow to calculate the shear stiffness of diaphragms fastened only along two edges from that of diaphragms fastened along all four edges. 


\section{Inhaltsverzeichnis}

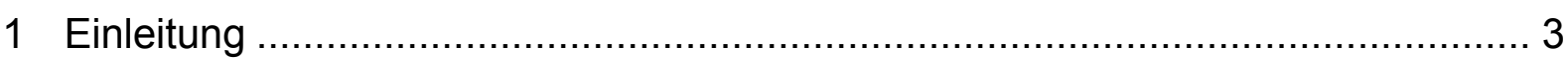

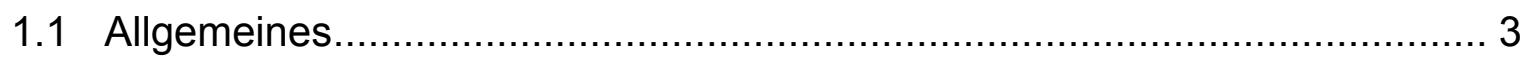

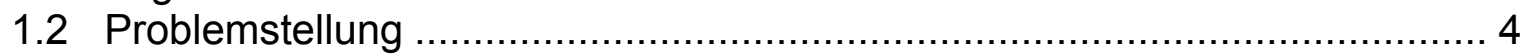

1.3 Ziele und Gliederung der Arbeit ................................................... 6

2 Darstellung der unterschiedlichen Stabilisierungsarten und Stand der Technik .... 7

2.1 Allgemeines................................................................................. 7

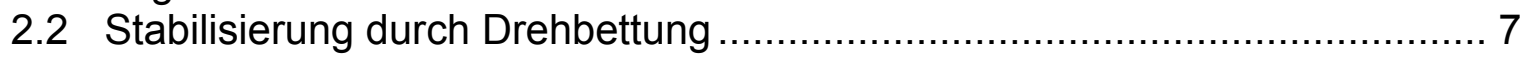

2.3 Stabilisierung durch Schubsteifigkeit.......................................... 11

3 Untersuchungen zur Stabilisierung durch Drehbettung ............................... 15

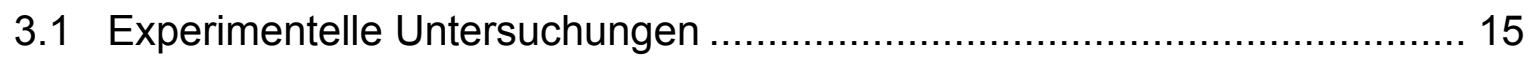

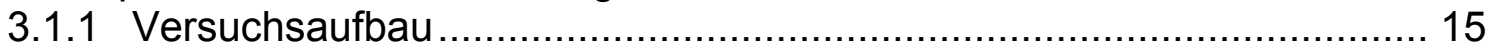

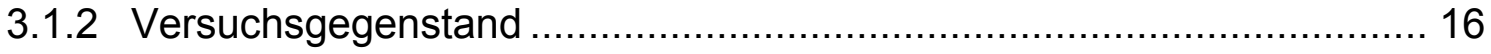

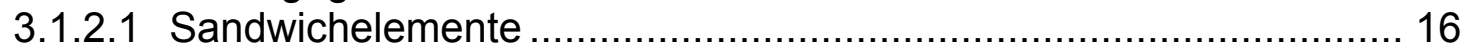

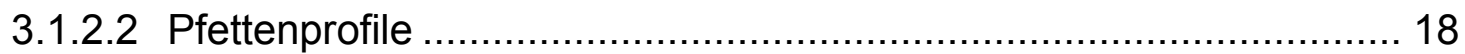

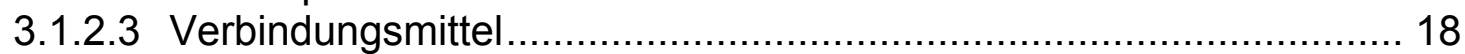

3.1.2.4 Zusammenstellung der Versuchsreihen ................................. 20

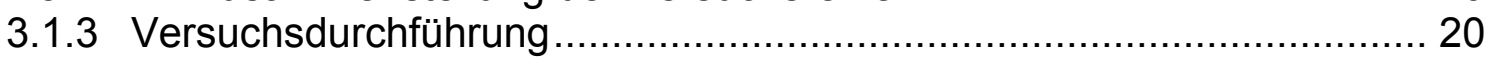

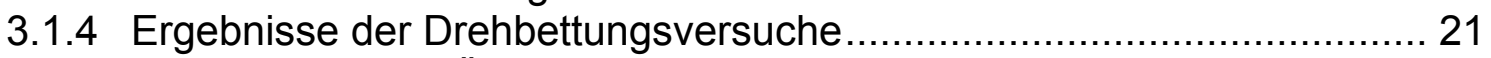

3.1.4.1 Tabellarische Übersicht ................................................... 21

3.1.4.2 Sandwichelemente mit Kernschicht aus Polyurethan-Hartschaum .. 22

3.1.4.3 Sandwichelemente mit Kernschicht aus Mineralfasern .................. 24

3.1.4.4 Untersuchungen zum Kriecheinfluss der PUR-Sandwichelemente .. 26

3.1.5 Untersuchungen zur Dichtigkeit der Elemente.................................. 27

3.1.6 Bestimmung der Materialkennwerte ............................................. 28

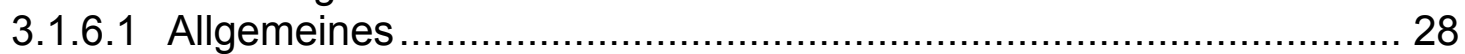

3.1.6.2 Mechanische Eigenschaften der Deckschichten ......................... 28

3.1.6.3 Mechanische Eigenschaften der Kernschichten ........................... 29

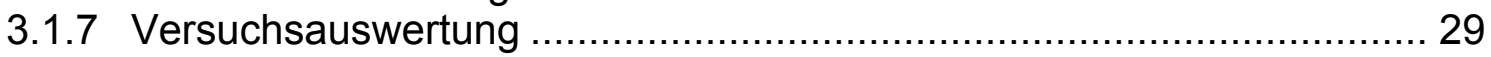

3.2 Berechnungen mit der Methode der Finiten Elemente ........................... 33

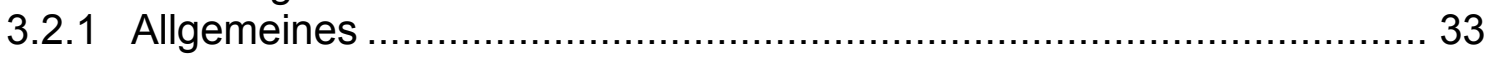

3.2.2 Beschreibung des Finite-Element-Modells................................. 33

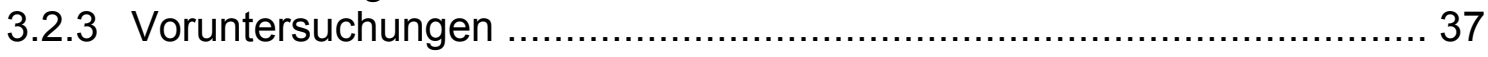

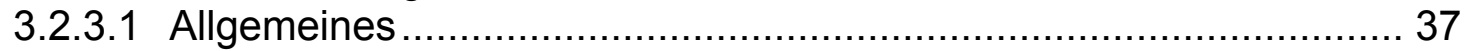

3.2.3.2 Biegetragverhalten des Sandwichelementes.............................. 37

3.2.3.3 Verformungsverhalten des Pfettenprofils.................................... 40

3.2.3.4 Werkstoffgesetz der Sandwichkernschicht ................................ 41

3.2.4 Verifizierung der Finite-Element-Modellierung ............................... 45

3.2.5 Berechnungen mit dem verifizierten Finite-Element-Modell zur

Bestimmung der signifikanten Einflüsse .................................... 49

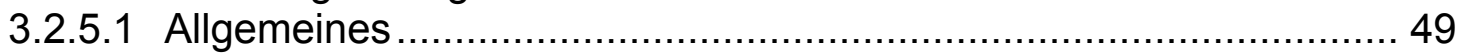

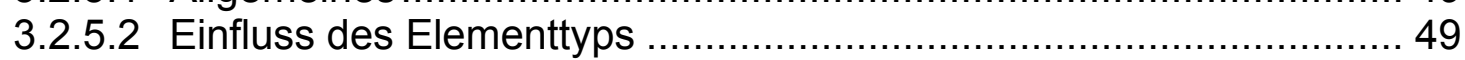

3.2.5.3 Einfluss der Pfettenauflast ................................................. 52

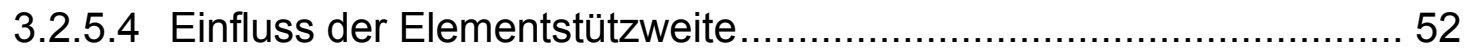

3.2.5.5 Einfluss des Elastizitätsmoduls der Sandwich-Kernschicht ............. 53

3.2.5.6 Einfluss der Elementdicke ..................................................... 55

3.2.5.7 Einfluss der Deckschichtdicken ........................................... 56

3.2.5.8 Einfluss der Obergurtbreite des Pfettenprofils ........................... 57 
3.2.5.9 Einfluss der Verbindungsmittelanordnung ................................ 58

3.2.5.10 Einfluss einer geänderten Profilform........................................ 59

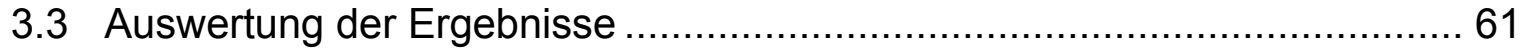

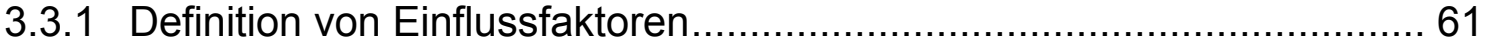

3.3.2 Statistische Auswertung der Versuchsergebnisse hinsichtlich $c_{\vartheta 1} \ldots \ldots \ldots 61$

3.3.2.1 Elemente mit Kernschicht aus Polyurethan-Hartschaum..................61 61

3.3.2.2 Elemente mit Kernschicht aus Mineralfasern .............................6 62

3.3.3 Statistische Auswertung der Versuchsergebnisse hinsichtlich $c_{\vartheta 2} \ldots \ldots \ldots 63$

3.3.3.1 Elemente mit Kernschicht aus Polyurethan-Hartschaum.................6 63

3.3.3.2 Elemente mit Kernschicht aus Mineralfasern ..............................63 63

3.3.4 Berücksichtigung des Kriecheinflusses bei PUR-Sandwichelementen .. 64

3.3.5 Berücksichtigung der Obergurtbreite und Anordnung der

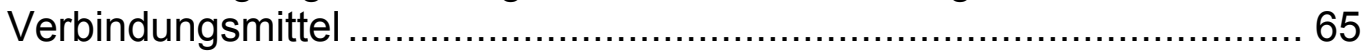

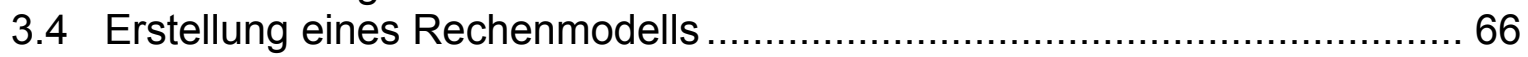

4 Untersuchungen zur Stabilisierung durch Schubsteifigkeit ...........................68

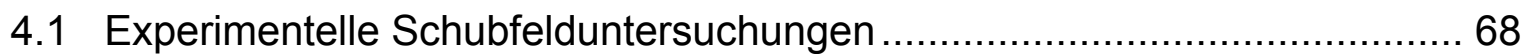

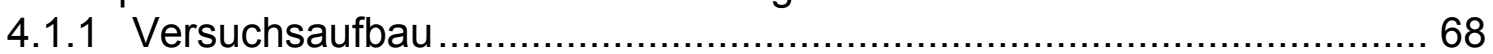

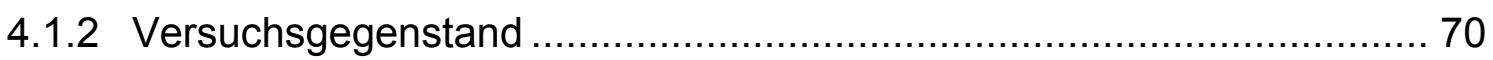

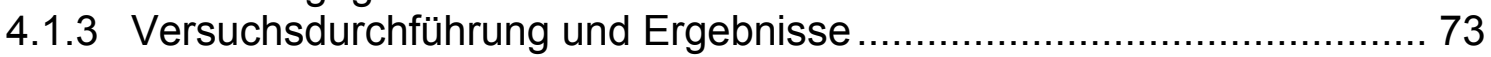

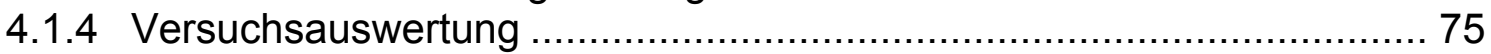

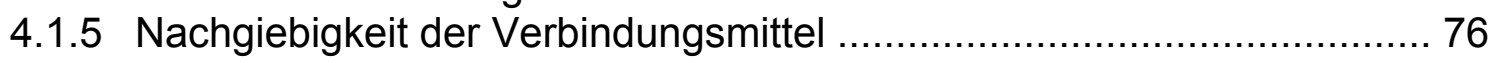

4.2 Berechnungen mit der Methode der Finiten Elemente .......................... 78

4.2.1 Beschreibung des Finite-Element-Modells .................................. 78

4.2.2 Nachgiebigkeit der Verbindungsmittel ........................................ 80

4.2.3 Verifizierung der Finite-Element-Modells mit Berechnungsergebnissen 81

4.2.4 Verifizierung des Finite-Element-Modells mit Versuchsergebnissen ..... 82

4.2.5 Berechnungen mit dem verifizierten Finite-Element-Modell zur

Bestimmung der signifikanten Einflüsse .................................... 85

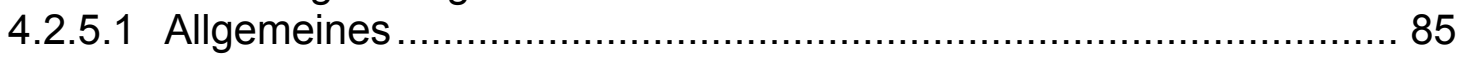

4.2.5.2 Berücksichtigung unterschiedlicher Profiltypen ........................... 85

4.2.5.3 Einfluss der Nachgiebigkeit der Verbindungsmittel an den Schubfeldrändern bei zwei- und vierseitiger Lagerung .................. 86

4.2.5.4 Einfluss der Nachgiebigkeit der Verbindungsmittel in den Längsstößen ........................................................................ 92

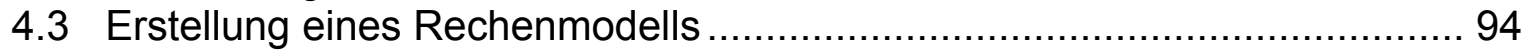

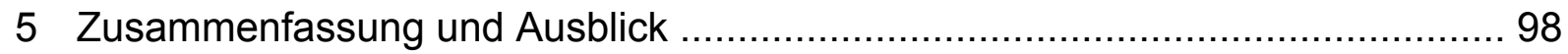

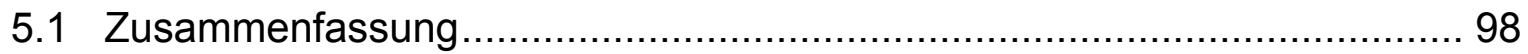

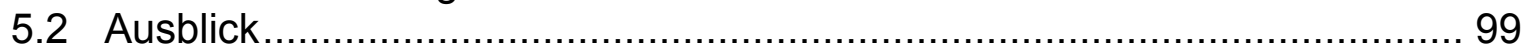

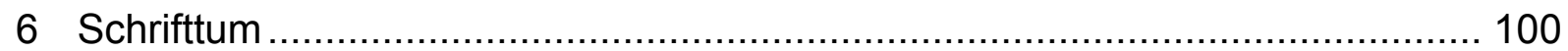

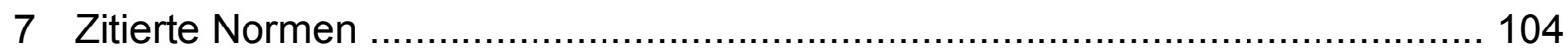

Anhang A1: Zusammenstellung der Versuchsreihen Drehbettung 105

Anhang A2: Momenten-Verdrehungsbeziehungen der Versuchsreihen Drehbettung 115

Anhang A3: Ergebnisse der Zugversuche an den Deckschichten $\quad 132$

Anhang A4: Ergebnisse der schaumtechnologischen Untersuchungen 133

Anhang A5: statistische Auswertungen $\quad 134$ 


\section{Einleitung}

\subsection{Allgemeines}

Im Zeichen einer sich wandelnden Baubranche sind große Tragfähigkeiten und vielfältigste Nutzungsmöglichkeiten, schnelle und kostengünstige Montagemöglichkeiten und die Recyclingeigenschaften die oftmals entscheidenden Kriterien bei der Auswahl moderner Baustoffe. Die Leichtbauweise - bestehend aus linienförmigen Tragkonstruktionen und tragenden, flächenhaften Elementen mit dünnwandigen, quasiebenen Blechen zum Raumabschluss - erfüllt diese Anforderungen in hervorragendster Weise. Zusätzlich zu den vorteilhaften funktionellen Eigenschaften zeichnen sich die Leichtbauelemente auch durch ihre vielseitigen Einsatzmöglichkeiten als Gestaltungselemente für Dächer und Fassaden aus.

Dadurch ergab sich der enorme Aufschwung dieser Bauweise in den letzten Jahrzehnten. Begünstigt wurde das stetige Wachstum zudem von der Entwicklung geeigneter Berechnungsmethoden, mit deren Hilfe sich die Tragsicherheit und Gebrauchstauglichkeit einer Tragkonstruktion mitsamt den eindeckenden Leichtbauelementen sicher nachweisen lässt. Im Hinblick auf die stetig steigenden Rohstoffpreise kann aufgrund der Wirtschaftlichkeit der Leichtbauweise mit einer weiteren Zunahme des Marktanteiles dieser Bauweise gerechnet werden. Hierzu werden sowohl die Hersteller von Leichtbauelementen durch neue Produktentwicklungen als auch die Tragwerksplaner durch die Ausnutzung erweiterter Berechnungsmethoden beitragen.

Die vorliegende Arbeit befasst sich mit den Möglichkeiten zur Nutzung der flächigen Dach- und Wandelemente aus dünnwandigem Blech im Hinblick auf eine Traglaststeigerung ihrer Unterkonstruktion. Damit sollen bestehende Berechnungsmethoden erweitert und eine wirtschaftlichere Dimensionierung von Stahlbauten ermöglicht werden.

Stellvertretend für die raumabschließenden Leichtbauelemente aus dünnwandigem Blech sind in Bild 1.1 verschiedene Geometrien von Trapezprofilen und in Bild 1.2 je ein Sandwichelement für den jeweils typischen Einsatzbereich Dach und Wand dargestellt. Weitere Typen können [25] und [26] entnommen werden.
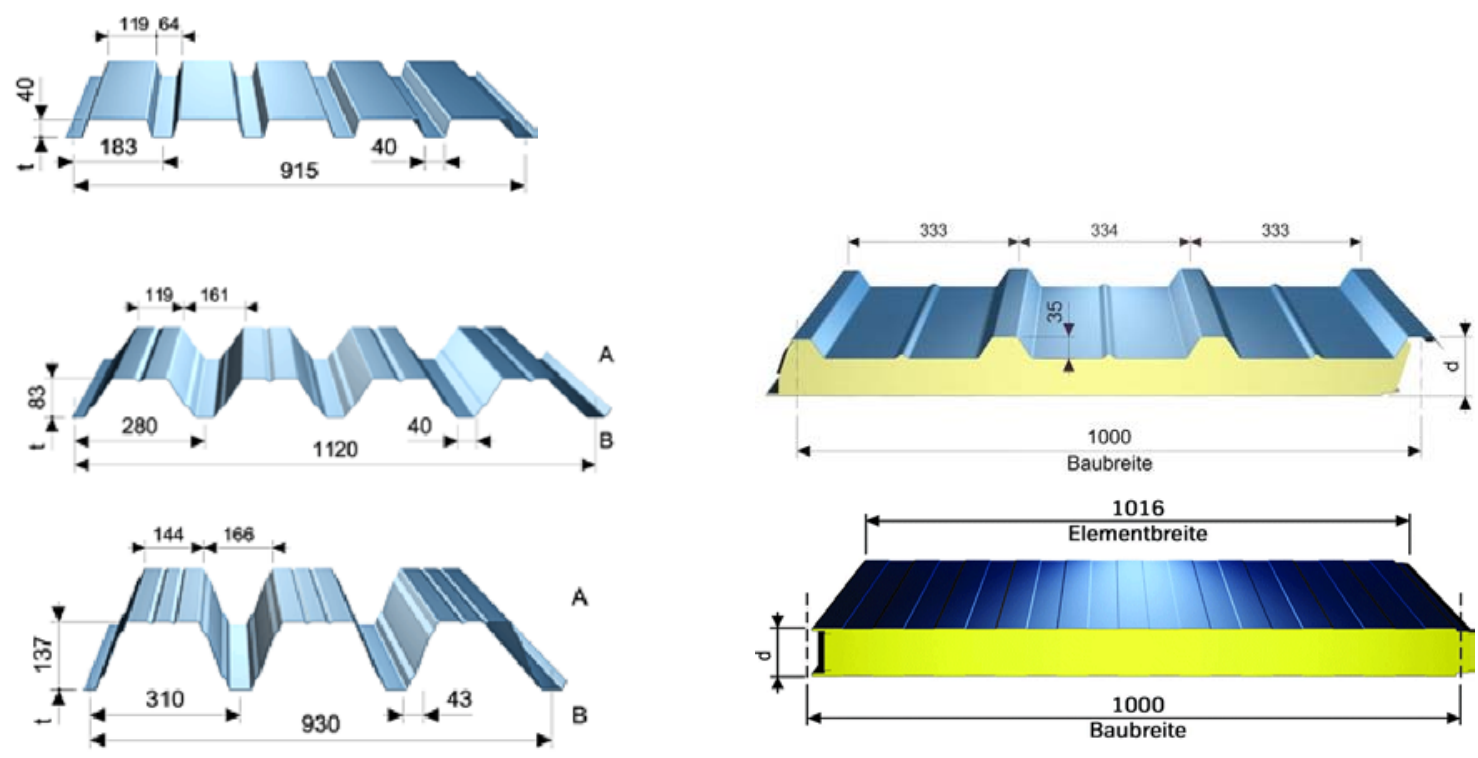

Bild 1.1: Trapezprofile

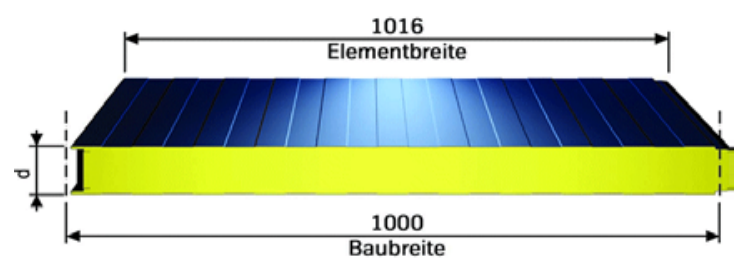

Bild 1.2: $\quad$ Sandwichelemente für Dach (oben) und Wand (unten) 
Die Formgebung der Trapezprofile erfolgt durch Rollformen von oberflächenveredeltem Blech mit Dicken bis zu 1,50 mm. Die dabei entstehenden Profilhöhen betragen bis zu $200 \mathrm{~mm}$, die Baubreiten der Profiltafeln liegen zwischen $750 \mathrm{~mm}$ und $1100 \mathrm{~mm}$. Sandwichelemente bestehen aus profilierten Deckschichten auf der Innenund Aussenseite und einer Kernschicht aus Polyurethan-Hartschaum oder Mineralfaserplatten.

Die aktuellen Methoden zur Berechnung und Ausführung von Stahltrapezprofilen sind in DIN 18807, Teile 1 und 3 in Verbindung mit [15] geregelt. Auf europäischer Ebene findet DIN EN 1993-1-3 Anwendung, die im wesentlichen mit den deutschen Regelungen übereinstimmt.

Anwendung, Bemessung und Ausführung von Sandwichelementen mit Metalldeckschichten werden aktuell in Deutschland durch allgemeine bauaufsichtliche Zulassungen des Deutschen Instituts für Bautechnik, Berlin, geregelt. Die europäische Regelung von Sandwichelementen erfolgt durch DIN EN 14509.

Weiterführende Informationen zur Planung, Konstruktion und Bauausführung mit Trapezprofilen und Sandwichelementen sind [32], [40] und [50] zu entnehmen.

\subsection{Problemstellung}

Für die Tragfähigkeit schlanker, stabförmiger Stahlbaukonstruktionen mit Druckbeanspruchung wird in vielen Fällen der Stabilitätsnachweis maßgebend. Dabei kommt dem mit Verdrehungen verbundenem seitlichen Ausweichen, das als Biegedrillknicken bezeichnet wird, eine besondere Bedeutung zu. Die Kopplung der beiden Ausweichmöglichkeiten reduziert die kritische Last entscheidend, wobei die kritischen Lasten für die beiden Komponenten der Ausweichverformung - wie auch die entsprechenden Steifigkeiten - ohnehin schon gering sind, da hierzu keine planmäßigen Lasten auftreten. Bild 1.3 zeigt die beim Versagensfall Biegedrillknicken auftretende Verschiebung v bei gleichzeitiger Verdrehung $\vartheta$ des Querschnitts.

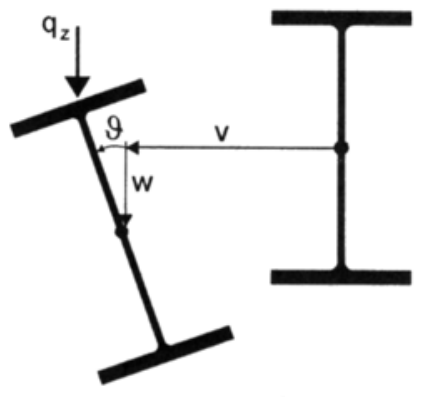

Bild 1.3: Querschnittsverformungen beim Biegedrillknicken

Die Behinderung dieser Verformungen durch die angrenzenden Bauteile kann die kritische Last deutlich steigern. Bei der Leichtbauweise handelt es sich bei den angrenzenden Bauteilen stets um ebene Flächentragwerke, die in der Regel über die gesamte Länge, in Ausnahmefällen auch über eine Teillänge mit dem zu stabilisierenden linienförmigen Bauteil verbunden sind. Die stabilisierende Wirkung ergibt sich aus der Biege- und/oder Scheibensteifigkeit der angrenzenden Elemente. Dies können Trapezprofile, Sandwichelemente, Deckbleche, Gitterroste und wenn man sich nicht auf die Leichtbauweise beschränkt, auch Mauerwerk oder Betonplatten sein. Im 
Rahmen dieser Arbeit wird in erster Linie die Stabilisierung durch Trapezprofile und Sandwichelemente behandelt.

Die stabilisierende Wirkung durch Behinderung der seitlichen Verschiebung des Querschnitts kann nach DIN 18800 Teil 2 und DIN EN 1993-1-3 für biegedrillknickgefährdete Träger durch die Scheibenwirkung angrenzender Trapezprofile berücksichtigt werden. Dieser Ansatz beruht auf analytischen Untersuchungen von I-Trägern mit kontinuierlicher seitlicher Stützung [21]. Das allgemeine Ergebnis dieser Untersuchung wurde in Bezug auf die Stabilisierung durch Trapezprofile in eine Mindestschubsteifigkeit S umgerechnet [33]. Besitzen die an den Träger angeschlossenen Trapezprofile mindestens diese Schubsteifigkeit, darf die Anschlussstelle als in Trapezblechebene unverschieblich angesehen werden.

Da jedoch alle Untersuchungen zur Stabilisierung durch die Scheibensteifigkeit der angrenzenden Bauteile aus rechentechnischen Gründen eine Lagerung längs aller vier Ränder voraussetzten, gelten die verfügbaren Berechnungsansätze auch nur für diesen Fall [4]. Wie Bild 1.4 und Bild 1.5 zeigen, ist eine solche allseitige Befestigung der Trapezprofile in einer üblichen Dach- oder Wandkonstruktion mit orthogonalen Traggliedern aufwendig und konstruktiv schwierig.

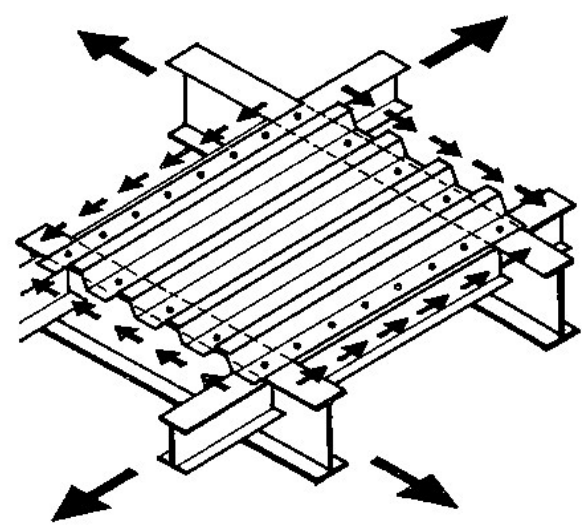

Bild 1.4: allseitige Befestigung durch zusätzliche Träger

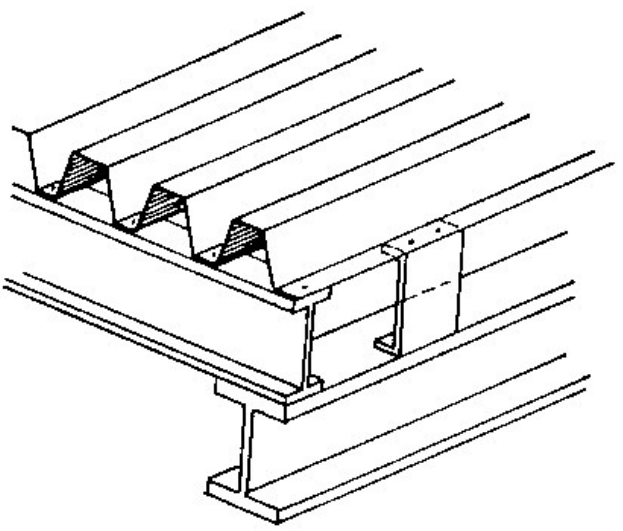

Bild 1.5: allseitige Befestigung durch zusätzliche Lasteinleitungsbleche

In der Praxis wird deshalb in der Regel auf eine umlaufende Befestigung verzichtet, und die Trapezprofile werden entsprechend ihrem Lastabtragungsverhalten lediglich an den zur Lastabtragungsrichtung rechtwinkligen Rändern an die Unterkonstruktion angeschlossen. Damit kann die vorteilhafte Wirkung der Schubbettung mangels Informationen über die Bettungswirkung rechnerisch nicht in Anspruch genommen werden.

Die Stabilisierung durch die Drehbettung einhüllender Bauteile darf nach DIN 18800 Teil 2 und DIN EN 1993-1-3 durch den Ansatz einer Drehfeder mit der Steifigkeit $\mathbf{c}_{\vartheta, \mathrm{k}}$ am gestützten Trägerflansch berücksichtigt werden. Die Steifigkeit $c_{\vartheta, k}$ wird dabei maßgeblich durch die Steifigkeit des Anschlusses zwischen Träger und stabilisierendem Bauteil bestimmt. Während für Stahltrapezprofile in DIN 18800-2 umfangreiche Angaben zu den Anschlusssteifigkeiten $\mathrm{C}_{\vartheta \mathrm{A}, \mathrm{k}} \mathrm{zu}$ finden sind, wird dort hinsichtlich der Anschlusssteifigkeiten von Sandwichelementen auf die Ergebnisse experimenteller Untersuchung lediglich zweier Dachelemente mit unterschiedlichen Befestigungsarten verwiesen [35]. Die daraus resultierenden Anschlusssteifigkeiten können jedoch 
wegen Abweichungen sowohl hinsichtlich der Elemente selbst als auch hinsichtlich der Befestigungsart und Unterkonstruktion nur selten zugrundegelegt werden. Zudem wurden die Untersuchungen mit sehr geringen Auflasten durchgeführt. Das Verhalten eines Systems aus Träger und Sandwichelement im Grenzzustand der Tragfähigkeit wurde im Rahmen dieser Untersuchung nicht betrachtet. Da das Biegedrillknicken jedoch gerade in diesem Bereich auszuschließen ist, muss die Belastung dementsprechend im Bereich der Grenztragfähigkeit gewählt werden.

\subsection{Ziele und Gliederung der Arbeit}

Das Ziel der Untersuchungen im Rahmen dieser Arbeit war es, die im vorigen Abschnitt aufgezeigten Lücken zur Berücksichtigung der stabilisierenden Wirkung von Stahltrapezprofilen und Sandwichelementen hinsichtlich der Bemessung biegedrillknickgefährdeter Träger zu schließen. Dies betrifft bei Stahltrapezprofilen mit zweiseitiger Lagerung die Stabilisierung durch Behinderung der seitlichen Verschiebung und bei Sandwichelementen die Stabilisierung durch Drehbettung.

In Kapitel 2 werden die Grundlagen der beiden oben genannten Stabilisierungsarten eines biegedrillknickgefährdeten Trägers sowie deren Herleitung und der aktuelle Stand der Technik näher erläutert.

Kapitel 3 beschreibt die an der Versuchsanstalt für Stahl, Holz und Steine an Sandwichelementen zur Ermittlung ihrer drehstabilisierenden Wirkung durchgeführten Bauteilversuche. Die zur Auswertung der Versuche notwendigen mechanischen Eigenschaften von Kern- und Deckschichten werden im Rahmen von Materialprüfungen bestimmt und den begleitend durchgeführten numerischen Berechnungen mit der Methode der Finiten Elemente zugrunde gelegt. Mit dem verifizierten FiniteElement-Modell werden Grundlagenuntersuchungen durchgeführt und die maßgebenden Einflussfaktoren im Hinblick auf die statistische Auswertung der Versuchsergebnisse bestimmt. Die Ergebnisse dieser Untersuchungen werden in einem allgemeingültigen Rechenmodell umgesetzt, mit dem die Anschlusssteifigkeit von Sandwichelementen in Abhängigkeit der Auflast bestimmt werden kann.

Kapitel 4 behandelt die stabilisierende Wirkung durch die Schubsteifigkeit zweiseitig gelagerter Trapezprofilscheiben. Dazu werden numerische Berechnungen mit einem Finite-Element-Modell durchgeführt, das sowohl durch Vergleich mit experimentellen Schubfeldversuchen als auch durch die Ergebnisse analytischer Berechnungen nach [52] verifiziert wird. Das Finite-Element-Modell berücksichtigt unterschiedlich große Nachgiebigkeiten der Verbindungsmittel, die zuvor in Querzugversuchen an verschiedenen Typen von Verbindungsmitteln ermittelt wurden. Das Kapitel schließt mit der Ermittlung eines Rechenmodells, das die Berechnung der Schubsteifigkeit eines zweiseitig gelagerten Schubfeldes ermöglicht.

Den Abschluss der Arbeit bildet Kapitel 5 mit einer Zusammenfassung der Ergebnisse und einem Ausblick auf weiterführende Forschungsmöglichkeiten. 


\section{Darstellung der unterschiedlichen Stabilisierungsarten und Stand der Technik}

\subsection{Allgemeines}

Entsprechend den aktuell geltenden technischen Regeln DIN 18800 Teil 2 und DIN EN 1993-1-3 kann die Stabilisierung eines Trägers durch den Ansatz von elastischen Federn berücksichtigt werden. Dabei wird die Drehbettungswirkung stabilisierender Bauteile durch eine Drehfeder mit der Steifigkeit $\mathrm{c}_{\vartheta}$ am Obergurt angesetzt, während die Stabilisierung gegen seitliches Ausweichen durch eine Wegfeder mit der Steifigkeit S berücksichtigt wird (siehe Bild 2.1).

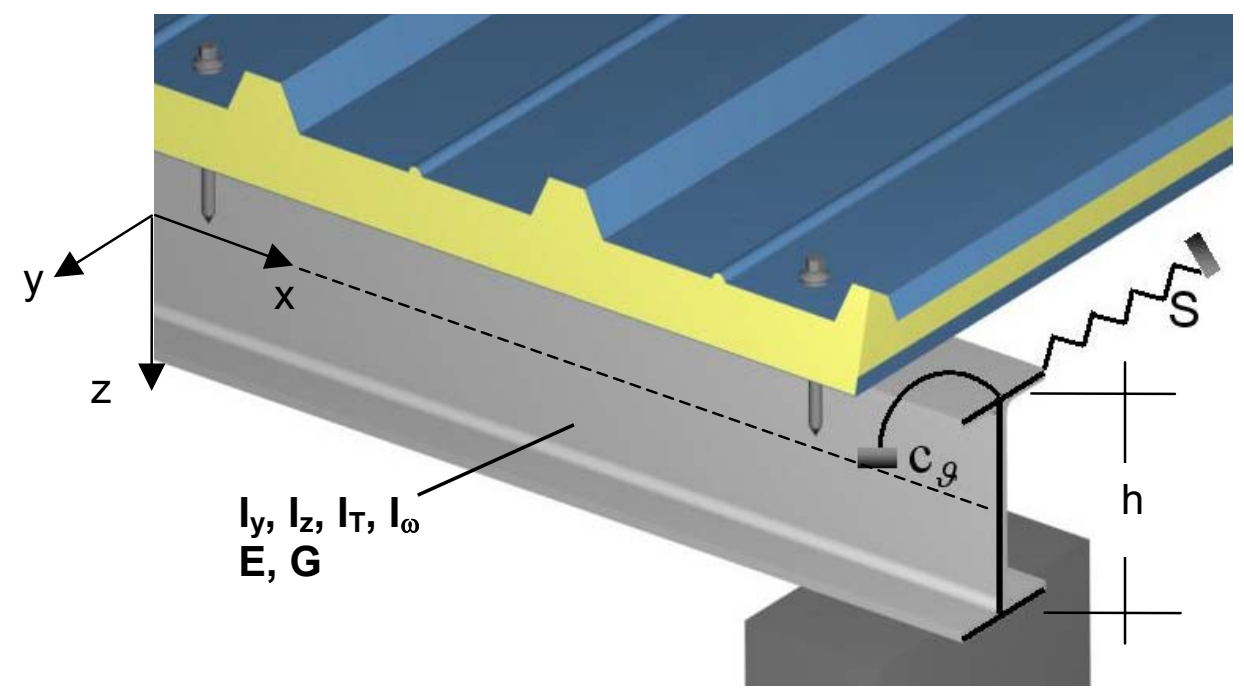

$\begin{array}{lll}\text { Querschnittsgrößen: } & \mathrm{I}_{\mathrm{y}}, \mathrm{I}_{\mathrm{z}} & \text { Flächenmoment 2. Grades } \\ & \mathrm{I}_{\mathrm{T}} & \text { Torsionswiderstandsmoment } \\ & \mathrm{I}_{\omega} & \text { Wölbflächenmoment 2. Grades } \\ & \text { h } & \text { Profilhöhe } \\ \text { Physikalische Kenngrößen: } & \mathrm{E} & \text { Elastizitätsmodul E } \\ & \mathrm{G} & \text { Schubmodul }\end{array}$

Bild 2.1: Federmodell zur Erfassung der Stabilisierung eines biegedrillknickgefährdeten Trägers

Im folgenden werden die zwei in Bild 2.1 dargestellten Stabilisierungsarten behandelt.

\subsection{Stabilisierung durch Drehbettung}

Die ersten theoretischen Untersuchungen zur Wirkung der kontinuierlichen Drehbettung auf die Biegedrillknicklast führte Chwalla am Beispiel eines Einfeldträgers mit Randmomenten durch [7]. Die Ergebnisse dieser Untersuchung wurden in die damals entstandenen Vorschriften zum Stabilitätsnachweis (DIN 4114) aufgenommen. Am Beispiel eines biege- und normalkraftbeanspruchten Zweifeldträgers mit kontinuierlicher Drehbettung zeigen Klöppel/Unger [31] mit ihren theoretischen Untersuchungen, dass für I-Träger eine Steigerung der ideellen Kippspannung $\sigma_{\mathrm{Ki}}$ um ein Vielfaches erreicht werden kann. 
In umfangreichen und aufwendigen Versuchen [42], [43], [44] wurde experimentell bestätigt, dass eindeckende Elemente, in diesen Fällen hauptsächlich Welleternitplatten, die Traglast stabilitätsgefährdeter Träger erheblich steigern. Die Traglaststeigerung wird dabei hauptsächlich der Biegesteifigkeit der drehbehindernden Dacheindeckung zugeschrieben.

Weiterführende theoretische Untersuchungen [47], [33] ergaben die Möglichkeit, die Drehbettung durch den Ansatz der Steifigkeit $c_{\vartheta}$ zusammen mit der Trägerlänge I bei beidseitiger Gabellagerung und dem Schubmodul G zur Berechnung einer vergrößerten Torsionssteifigkeit $\mathrm{I}_{\mathrm{T}}{ }^{*}$ nach Gleichung (2.1)

$$
I_{T}^{*}=I_{T}+c_{\vartheta} \cdot l^{2} /\left(\pi^{2} \cdot G\right)
$$

zu berücksichtigen. Nach [29] und [30] werden die mit $\mathrm{I}_{\mathrm{T}}{ }^{*}$ berechneten Biegedrillknickmomente jedoch teilweise erheblich überschätzt.

Alternativ dazu lässt sich nach [34] unter Berücksichtigung einer kontinuierlichen Drehbettung $\mathrm{c}_{\vartheta}$ für gabelgelagerte Träger mit sehr großen Stützweiten das ideale Biegedrillknickmoment $M_{k i}$ mit Gleichung (2.2) berechnen

$$
M_{K i}^{2}=\frac{k^{2}}{\pi^{2}} \cdot E I_{z} \cdot c_{\vartheta}
$$

wobei k ein vom Momentenverlauf abhängiger Beiwert ist, der beispielsweise [33] zu entnehmen ist.

Die Mindeststeifigkeit einer konstanten Drehbettung ist danach erreicht, wenn 95\% des Biegemomentes $\mathrm{M}_{\mathrm{pl}}$ im vollplastischen Zustand erreicht werden. Dies kann durch den Abminderungsfaktor $\kappa_{M}$

$$
\kappa_{m}=\left(\frac{1}{1+{\overline{\lambda_{M}}}^{2 n}}\right)^{\frac{1}{n}}=0,95
$$

mit dem Trägerbeiwert n nach DIN 18800 Teil 2 und der bezogenen Schlankheit

$$
\bar{\lambda}_{M}=\sqrt{\frac{M_{p l}}{M_{K i}}}
$$

berücksichtigt werden. Nach Rückrechnung über den zugehörigen Schlankheitsgrad $\bar{\lambda}_{M}$ ergibt sich entsprechend Gleichung (2.5) die erforderliche Drehbettungssteifigkeit zu

$$
\operatorname{erf} c_{\vartheta}=\frac{M_{p l}^{2}}{E I_{z}} \cdot k_{\vartheta}
$$

Die zur Berechnung der erforderlichen Drehbettungssteifigkeit notwendigen Beiwerte $\mathrm{k}_{\vartheta}$ sind in Abhängigkeit des vorliegenden Momentenverlaufs für freie und am Druckgurt gebundene Drehachse in Tabelle 2.1 dargestellt. 


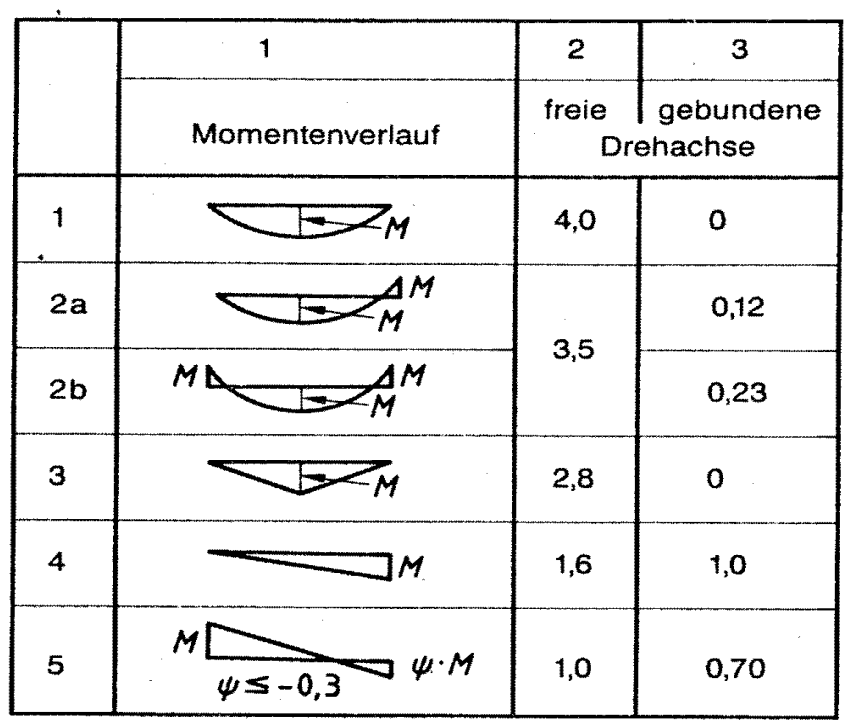

Tabelle 2.1: Beiwerte $k_{\vartheta}$ aus DIN 18800 Teil 2

Sowohl Gleichung (2.5) als auch Tabelle 2.1 sind aus DIN 18800 Teil 2 entnommen.

Die Drehbettung $\mathrm{c}_{\vartheta}$ ergibt sich als Widerstand der angrenzenden Bauteile gegen ein Verdrehen des Trägers. In den ersten Untersuchungen zur Bestimmung der Drehbettung wurde zunächst ausschließlich die Biegesteifigkeit der angrenzenden Bauteile betrachtet [47] [54]. Die dazu getroffene Annahme, dass insbesondere eine starre Verbindung zwischen dem zu stützenden Träger und dem angrenzenden Bauteil vorliegt, trifft jedoch beim Anschluss von Elementen des Leichtbaus nicht zu. Zusätzlich zu Nachgiebigkeiten aus dem Anschluss können Nachgiebigkeiten aus der Profilverformung zu berücksichtigen sein.

Dieser Sachverhalt kann durch ein System hintereinander geschalteter Federn entsprechend Gleichung (2.6) berücksichtigt werden [33] [37].

$$
\frac{1}{c_{\vartheta, k}}=\frac{1}{c_{\vartheta M, k}}+\frac{1}{c_{\vartheta A, k}}+\frac{1}{c_{\vartheta P, k}}
$$

mit $\quad \mathrm{C}_{\vartheta \mathrm{M}, \mathrm{k}}$ Drehbettung aus der Biegesteifigkeit des abstützenden Bauteils unter Annahme einer starren Verbindung

$$
\begin{array}{rll}
c_{\vartheta M, k}=k \cdot \frac{E I_{D}}{a} & \multicolumn{1}{c}{(2.7)} \\
\text { mit } & \mathrm{k}=2 & \text { für Ein- und Zweifeldträger } \\
& \mathrm{El}_{\mathrm{D}} & \text { Biegesteifigkeit des abstützenden Bauteils } \\
\mathrm{a} & \text { Stützweite des abstützenden Bauteils }
\end{array}
$$

$\mathrm{C}_{\vartheta} \mathrm{P}, \mathrm{k}$ Drehbettung aus der Profilverformung des gestützten Trägers

$\mathrm{C}_{\vartheta} \mathrm{A}, \mathrm{k}$ Drehbettung aus der Anschlusssteifigkeit

Die Anteile der Drehbettung aus der Profilverformung können nach [34] berechnet werden. Ist der Wert $\mathrm{c}_{\vartheta}, \mathrm{k}$ aus experimentellen Untersuchungen am Gesamtsystem bekannt, kann die Anschlusssteifigkeit $\mathrm{C}_{\vartheta} \mathrm{A}, \mathrm{k}$ mit Gleichung (2.6) ermittelt werden. Die im Rahmen von [33] ermittelten und in DIN 18800 Teil 2 für unterschiedliche Ausfüh- 
rungsvarianten aufgeführten Anschlusssteifigkeiten von Trapezprofilen basieren auf diesem Verfahren.

Auch die wenigen bisher bekannten Anschlusssteifigkeiten $\mathrm{C}_{\vartheta} \mathrm{A}, \mathrm{k}$ von Sandwichelementen wurden mit diesem Verfahren ermittelt. Im Rahmen von Versuchen an Dachdeckungen mit unterlegter Wärmedämmung wurden die in Tabelle 2.2 aufgeführten und in [4] zitierten Anschlusssteifigkeiten $c_{\vartheta} A, k$ für zwei Dachelemente mit unterschiedlichen Befestigungsarten ermittelt [35].

\begin{tabular}{c|c|c|c|c}
\hline b) & Art der Befestigung & $\begin{array}{c}\text { Obergurt } \\
e=2 b_{r} \\
\text { mit Ka- } \\
\text { lotten }\end{array}$ & $\begin{array}{c}\text { Obergurt } \\
e=2 b_{r} \\
\text { mit } \\
\text { Dicht- } \\
\text { scheiben } \\
\varnothing 22\end{array}$ & $\begin{array}{c}\text { Untergurt } \\
e=2 b_{r} \\
\text { mit } \\
\text { Dicht- } \\
\text { scheiben } \\
\varnothing 22\end{array}$ \\
\hline $1 \quad \begin{array}{c}\text { HOESCH isodach } \\
\text { TL 75 }\end{array}$ & 2,2 & 2,1 & 1,8 \\
2 & $\begin{array}{c}\text { HOESCH isodach } \\
\text { TL 95 }\end{array}$ & 2,5 & 2,6 & 2,3 \\
\hline
\end{tabular}

Tabelle 2.2: Anschlusssteifigkeiten $c_{\vartheta A, k}$ von Sandwichelementen [4], Werte in $\mathrm{kNm} / \mathrm{m}$

Der zur Berechnung der Anschlusssteifigkeiten erforderliche Wert $c_{\vartheta, k}$ wurde dabei aus der am Gesamtsystem aufgezeichneten Momenten-Verdrehungsbeziehung als Sekantensteifigkeit bei einer definierten Verdrehung $\vartheta=0,1$ rad bestimmt. Die Anschlusssteifigkeiten nach Tabelle 2.2 sind unabhängig von der Obergurtbreite des zu stabilisierenden Trägers, gelten jedoch ausschließlich für die geprüften Elemente und angegebenen Befestigungsarten. Vergleichbare Untersuchungen an Trapezprofilen haben gezeigt, dass die Anschlusssteifigkeiten durch große Pfettenbelastungen deutlich gesteigert werden können [36]. Ungeachtet dieser Feststellung basieren die Werte nach Tabelle 2.2 jedoch auf Untersuchungen mit relativ geringen Auflasten.

Eine andere Abschätzung der drehbettenden Wirkung von Sandwichelementen ist in [45] zu finden. Im Rahmen dieser Untersuchung wird die Wirkung der unteren Deckschicht des Sandwichelementes vernachlässigt und lediglich die stützende Wirkung des Schaumkerns angesetzt. Die Abschätzung der Drehbettung erfolgt gemäß Bild 2.2 unter Annahme einer konstanten Dehnung über die Dicke des Elements.

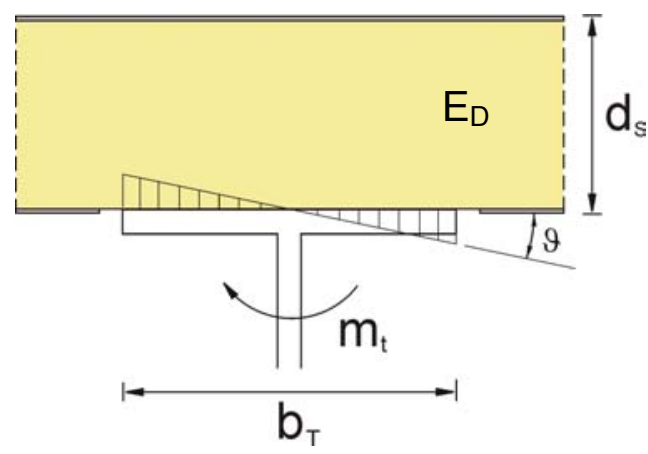

Bild 2.2: System zur Abschätzung der Drehbettung [45] 
Daraus ergibt sich der allgemeingültige Ansatz für die Größe der Drehbettung zu

$$
\mathrm{c}_{\vartheta}=\frac{\mathrm{E}_{\mathrm{D}} \cdot \mathrm{b}_{\mathrm{T}}{ }^{3}}{12 \cdot \mathrm{d}_{\mathrm{S}}}
$$

mit $\quad E_{D}$ Druckmodul des Schaumkerns

$\mathrm{b}_{\mathrm{T}} \quad$ Obergurtbreite des zu stabilisierenden Trägers

$d_{S} \quad$ Dicke des durchgehenden Schaumkerns

Der Druckmodul des Schaumkerns wird dabei als Tangentenmodul bei größeren Belastungen einheitlich mit $E_{s}=2,0 \mathrm{~N} / \mathrm{mm}^{2}$ angenommen.

Im Gegensatz zu den Untersuchungen nach [35], wo erstaunlicherweise festgestellt wurde, dass gegensätzlich zu den Trapezprofilen bei den Sandwichelementen die Werte $\mathrm{c}_{\vartheta} \mathrm{A}, \mathrm{k}$ unabhängig von der Obergurtbreite des zu stabilisierenden Trägers sind, hat hierbei die Obergurtbreite einen maßgebenden Einfluss. Die Berechnung der Anschlusssteifigkeit nach Gleichung (2.8) setzt auch in ausgelenkter Stellung einen vollständigen flächigen Kontakt über die gesamte Obergurtbreite des Trägers voraus. Dies muss durch eine ausreichend große Auflast sichergestellt sein. Kommt es zu einem Verlust des ganzflächigen Kontaktes zwischen Obergurt und Sandwichelement, wird die Anschlusssteifigkeit durch die Gleichung (2.8) überschätzt.

In [28] wurde die Drehbettung von Sandwichelementen mit der Methode der Finiten Elemente untersucht. Das numerische Modell beschränkt sich auf die Abbildung des Anschlussbereiches eines Sandwichelementes und berechnet den Widerstand des Elementes gegen Eindrücken eines Schraubenkopfes mitsamt Dichtscheibe. Die aus der Berechnung resultierende lineare Federsteifigkeit wird in eine Drehfedersteifigkeit umgerechnet und mit experimentellen Untersuchungen nach DIN EN 1993-1-3 verifiziert. Die Wirkung von Sog- oder Auflast wird im Rahmen der Untersuchungen lediglich durch die fragwürdige Änderung des Pfettendrehsinns berücksichtigt. Der günstige Effekt einer realitätsnahen Auflast wird auch bei diesen Untersuchungen nicht berücksichtigt. Eine Verallgemeinerung der in [28] aufgeführten Ergebnisse ist nicht möglich, da diese lediglich für bestimmte Parameter ermittelt wurden und keine Umsetzung in ein Rechenmodell erfolgte.

\subsection{Stabilisierung durch Schubsteifigkeit}

Aus theoretischen Untersuchungen an Einfeld- und Durchlaufträgern [21], [31] ist bekannt, dass bereits geringe Steifigkeiten einer seitlichen Stützung für eine deutliche Steigerung der Biegedrillknicklast ausreichend sind. Bei zunehmender Steifigkeit der seitlichen Stützung nähern sich die ideellen Kippspannungen asymptotisch dem Wert, der sich für den Fall einer gebundenen Drehachse ergibt. In [21] werden für Einfeld- und Durchlaufträger Mindeststeifigkeiten seitlicher Stützungen berechnet, mit denen 95\% der idealen Verzweigungslast bei gebundener Drehachse erreicht werden.

Die Übertragung der Erkenntnisse aus [21] hinsichtlich der seitlichen Stabilisierung durch Schubfelder aus Trapezprofilen erfolgt in [33] und ist in DIN 18800 Teil 2 über- 
nommen. Erfüllt danach die Schubsteifigkeit S einer Scheibe aus Trapezprofilen die nachfolgende Bedingung

$$
S \geq\left(E I_{\varpi} \frac{\pi}{l^{2}}+G I_{T}+E I_{z} \frac{\pi^{2}}{l^{2}} \cdot 0,25 h^{2}\right) \cdot \frac{70}{h^{2}}
$$

so darf die Anschlussstelle als in Trapezprofilebene unverschieblich gehalten angesehen werden. Liegt der Anschluss des Trapezprofils dabei am Druckgurt, führt diese gebundene Drehachse zu einer enormen Steigerung der Biegedrillknicklast. Dieser Sachverhalt wird durch Tabelle 2.1 bestätigt, da die daraus ersichtlichen Beiwerte $\mathrm{k}_{\vartheta}$ bei einer am Druckgurt gebundenen Drehachse die erforderliche Mindeststeifigkeit nach Gleichung (2.5) deutlich vermindern.

Neuere Erkenntnisse [23], [55] auf Grundlage analytischer Untersuchungen belegen, dass schon wesentlich kleinere Steifigkeiten als die nach Gleichung (2.9) ausreichend sind, um die maximal aufnehmbare Last nach Theorie I. Ordnung zu erreichen. Bereits kleine Schubfeldwerte bewirken einen hohen Anstieg der Tragfähigkeit. Die Forderung einer gebundenen Drehachse ist dazu nicht zwingend erforderlich.

Diese analytische Feststellung wird in [48] durch begleitende experimentelle Untersuchungen und Finite-Element-Berechnungen bestätigt. Die erforderliche Schubsteifigkeit $S$ bei deren Vorliegen Einfeld- oder Durchlaufträger mit Schlankheiten $0,4<\lambda_{M}<1,0$ ohne zusätzliche Drehbettung ausreichend stabilisiert werden können, kann danach mit Gleichung (2.10) berechnet werden.

$$
\mathrm{S}=\frac{2\left(\pi^{2}+3\right)^{2}}{3 \pi^{2}\left(\pi^{2}-3\right)} \cdot \frac{\mathrm{M}_{\mathrm{pl}}}{\bar{\lambda}_{\mathrm{M}}{ }^{2} \mathrm{~h}}-\frac{3 \pi^{2}}{\pi^{2}-3} \cdot \frac{\mathrm{EI} \mathrm{z}}{\mathrm{l}^{2}} \cdot\left[-1+\sqrt{1+\left(\frac{\pi^{2}+3}{3 \pi}\right)^{2} \frac{\mathrm{c}^{2}}{\mathrm{~h}^{2}}}\right]
$$

wobei

$$
c^{2}=\frac{\pi^{2} E I_{\omega}+G I_{T} l^{2}}{E I_{z}}
$$

Die Schubsteifigkeit von Trapezprofilen wird von Schardt/Strehl [52], [49] nach der Elastizitätstheorie bestimmt. Basierend auf der Faltwerkstheorie wird unter Annahme eines umlaufenden, konstanten Schubflusses das elastische Verformungsverhalten der Rippen unter Schubbeanspruchung bestimmt und unter Berücksichtigung der sich einstellenden Winkeländerung aus Schubverzerrung und Blechendverwölbung ein ideeller Schubmodul $\mathrm{G}_{S}$ abgeleitet. Die Berechnung des Schubmoduls kann entsprechend Gleichung (2.12) über die Kennwerte $\mathrm{K}_{1}$ und $\mathrm{K}_{2}$ erfolgen, die den Typenblättern der Trapezprofilhersteller für verschiedenste Profilgeometrien zu entnehmen sind, zum Beispiel [39].

$$
G_{S}=\frac{10000}{K_{1}+\frac{K_{2}}{l_{S}}}
$$

mit Is Schubfeldlänge entsprechend Bild 2.3 


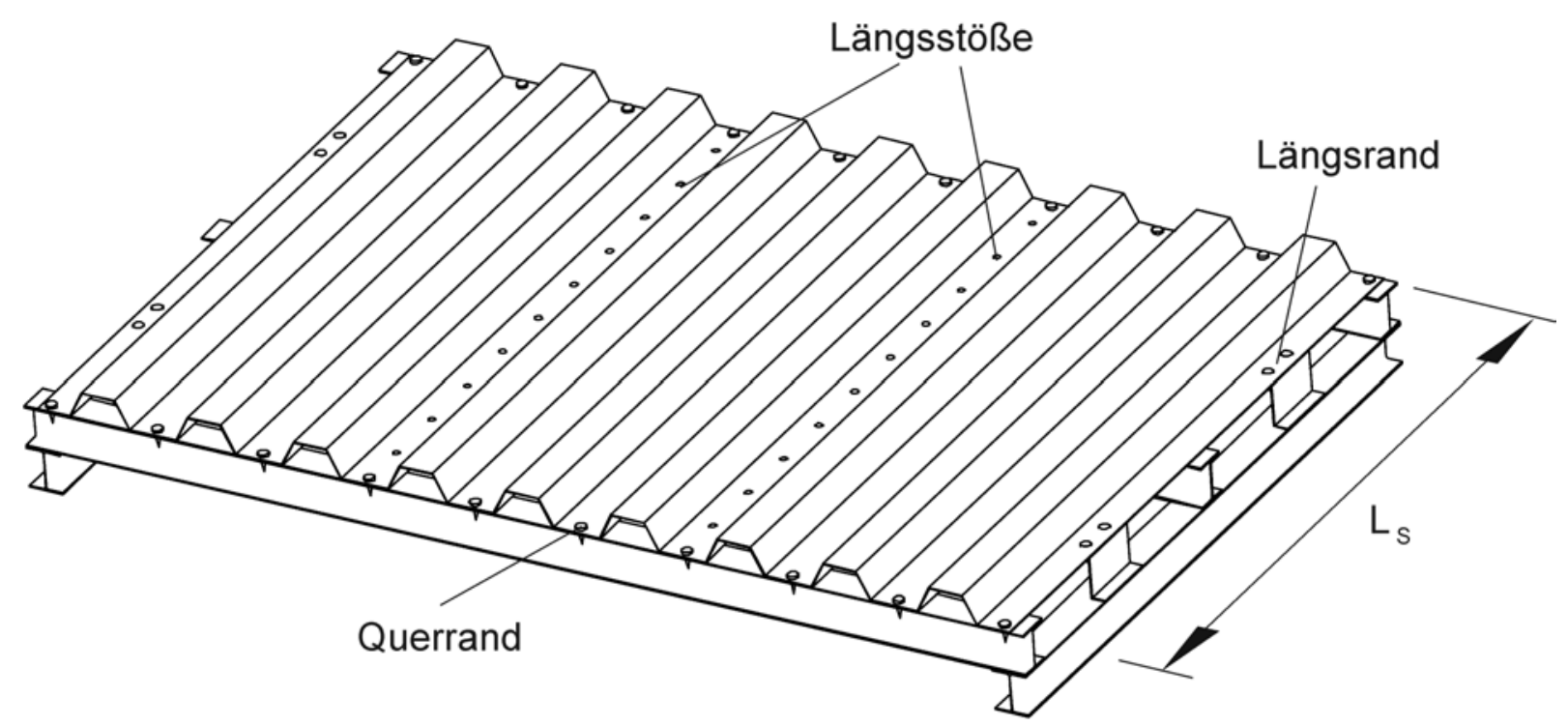

Bild 2.3: beispielhafte Ausführung eines Schubfeldes mit umlaufender Befestigung

Die für einen Träger verfügbare Schubsteifigkeit einer Trapezprofilscheibe wird nach Gleichung (2.13) ermittelt.

$$
\operatorname{vorh} S=G_{S} \cdot \frac{l_{S}}{n}
$$

mit $\quad \mathrm{n} \quad$ Anzahl der durch das Schubfeld auszusteifenden Träger

Mit dieser Schubsteifigkeit vorh $S$ ist der Nachweis einer ausreichenden seitlichen Stabilisierung in Verbindung mit Gleichung (2.13) einfach zu führen.

Überlegungen zur Schubfeldberechnung von Trapezprofilen nach Baehre/Wolfram [2], die auf der Schubfeldtheorie von Bryan/Davies [8] basieren und in den Grundzügen mit dem Verfahren Schardt/Strehl vergleichbar sind, liefern unter Berücksichtigung der Verformungen des Trapezblechs (Blechendverwölbung und Schubverzerrung) in etwa gleich große Werte für die Schubsteifigkeit S. Zusätzlich können bei diesem Verfahren auch die Nachgiebigkeiten aus den Verbindungen und den Randträgern berücksichtigt werden, womit die Gesamtverformung eines Schubfeldes ermittelt werden kann. In experimentellen Untersuchungen [57b] wurde gezeigt, dass diese Nachgiebigkeiten einen erheblichen Einfluss auf die Schubsteifigkeit haben und deren Vernachlässigung zu einer Überschätzung der Schubsteifigkeit führen.

Bei allen zuvor aufgeführten Untersuchungen und Nachweisen wird entsprechend Bild 2.3 von einem Schubfeld ausgegangen, das entsprechend DIN 18807 Teil 2 in Verbindung mit [4] an allen vier Rändern mit der Unterkonstruktion verbunden ist. Trapezprofilscheiben mit Befestigung in jeder zweiten Profilrippe dürfen gemäß DIN 18807 durch eine auf 20\% reduzierte Schubsteifigkeit S angesetzt werden.

Beispielhafte Untersuchungen zur stabilisierenden Wirkung durch zweiseitig gelagerte Trapezprofile wurden in [38] vorgestellt. Dabei wurden sinusförmige Querbelastungen zur Simulation von Abtriebskräften auf Träger aufgebracht, deren seitliche Ausweichmöglichkeit durch den Anschluss von zweiseitig gelagerten Trapezblechen behindert war. Auf diese Art konnte die stabilisierende Wirkung für die untersuchten Einzelfälle nachgewiesen werden. Zusätzlich zu den Versuchen wurden numerische Berechnungen an zwei unterschiedlichen Typen von Trapezprofilen durchgeführt. 
Diese beispielhaften Berechnungen sollen den Einfluss bestimmter Parameter auf die Schubsteifigkeit verdeutlichen. Da kein mechanisches Modell zur Erklärung der Tragweise eines zweiseitig gelagerten Schubfeldes betrachtet wird, fehlt den Untersuchungen bei der Vielzahl der Parameter die notwendige Systematik, wodurch keine Berechnungsvorschrift zur Verfügung gestellt werden kann. Einzelne Berechnungsergebnisse aus [38] können jedoch zum Vergleich mit den Ergebnissen dieser Arbeit herangezogen werden.

Im Hinblick auf das Tragverhalten ist bei zweiseitig gelagerten Trapezprofilscheiben ein abweichendes Verhalten gegenüber dem eines allseitig gelagerten Schubfeldes zu erwarten. Aufgrund der fehlenden Befestigung am Längsrand wird sich im Gegensatz zur vierseitigen Lagerung zumindest in diesem Bereich kein konstanter Schubfluss einstellen können. Die Anteile des Schubflusses, die bei der umlaufenden Lagerung über die Längsränder in die Unterkonstruktion abgetragen werden, müssen im Fall der zweiseitigen Lagerung zusätzlich durch die Verbindungsmittel am Querrand aufgenommen werden. Mit diesen wesentlichen Unterschieden im Tragverhalten kann mit den bisher existierenden Regeln weder eine Aussage zur Schubsteifigkeit noch zur Tragfähigkeit des Schubfeldes getroffen werden. 


\section{Untersuchungen zur Stabilisierung durch Drehbettung}

\subsection{Experimentelle Untersuchungen}

\subsubsection{Versuchsaufbau}

Der Aufbau zur Durchführung der Drehbettungsversuche wurde in Anlehnung an [35] und [36] als Dachausschnitt konzipiert und in den zweiaxialen Prüfstand (Fabrikat Messtek) der Versuchsanstalt für Stahl, Holz und Steine der Universität Karlsruhe (TH) integriert (siehe Bild 3.1).

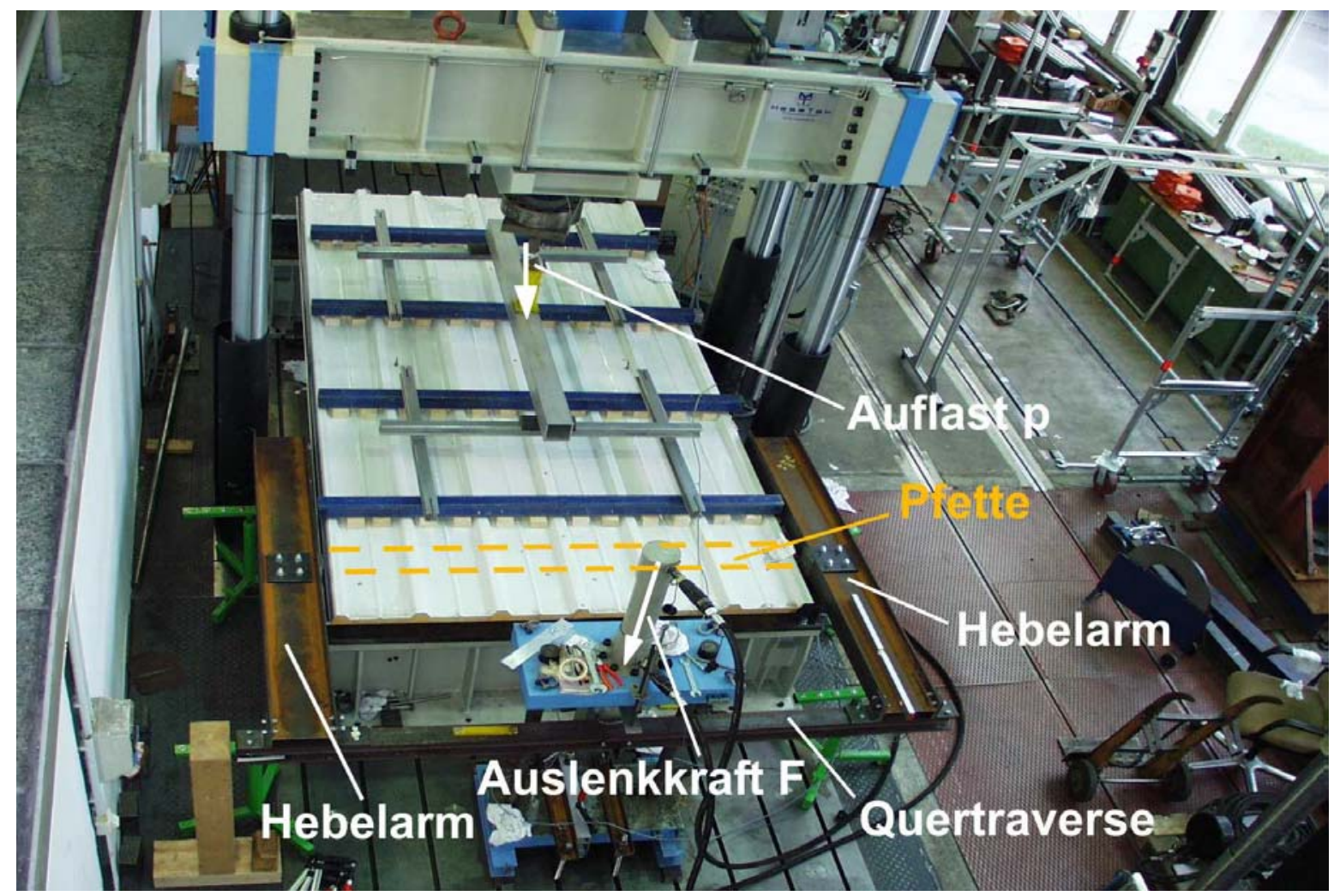

Bild 3.1: Versuchsaufbau [17]

Der Versuchsaufbau besteht aus einer Pfette, die beidseitig drehbar gelagert ist und als Randträger eines Feldes mit der Stützweite $I_{s}$ mit zwei nebeneinanderliegenden Sandwichelementen eingedeckt und verschraubt wird. Die Sandwichelemente werden mit Hilfe von lastverteilenden Trägern mit der Auflast $p$ flächig vorbelastet.

Damit aus dem Versuchsaufbau weder Zwängungen noch Widerstände gegen das Verdrehen der Pfette auftreten, wurden an den Auflagern der Pfette PräzisionsPendelkugellager und am zweiten Auflagerpunkt der Sandwichelemente Gleitlager verwendet. Über Kopfplatten an den Pfettenenden sind rechtwinklig zur Pfettenlängsachse Hebelarme angebracht, die durch eine Quertraverse miteinander verbunden sind. Die Auslenkung des Systems erfolgt durch einen weggesteuerten Hyraulikzylinder, der die Quertraverse mit der Auslenkkraft $F$ belastet. Die Auslenkkraft $F$ wird durch eine Kraftmessdose, Typ HBM Z3H2 mit 5 kN Maximalbelastung, gemessen. Eine Prinzipskizze des Versuchsaufbaus in seitlicher Ansicht ist in Bild 3.3 dargestellt. 


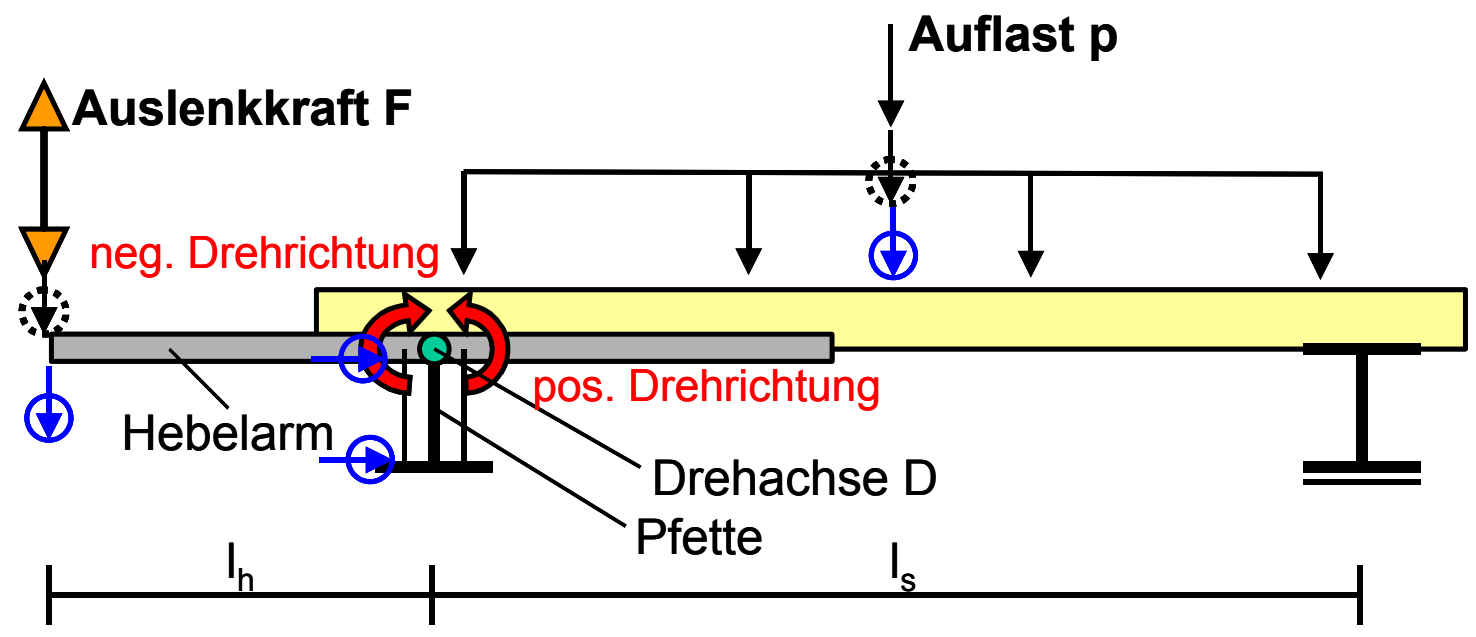

\section{Verformungsmessungen \\ Kraftmessungen}

Bild 3.2: Prinzipskizze des Versuchsaufbaus in seitlicher Ansicht

Die aus der Drehbewegung um die Achse $D$ resultierenden Verschiebungen $v_{o}$ und $v_{u}$ von Ober- und Untergurt (siehe Bild 3.3) werden mit Seilzugwegaufnehmern gemessen und mit Gleichung (3.1) in eine zugehörige Verdrehung umgerechnet.

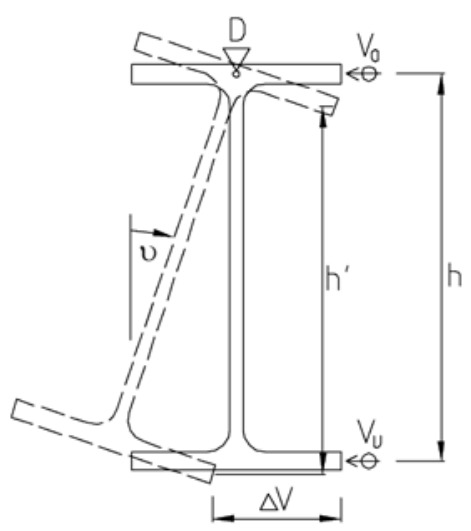

Bild 3.3: Drehpunkt $D$ und Verschiebungen $v_{o}$ und $v_{u}$

$$
\vartheta_{M}=\arctan \left(\frac{\Delta v_{o}-\Delta v_{u}}{h^{\prime}}\right)=\arcsin \left(\frac{\Delta v_{o}-\Delta v_{u}}{h}\right)
$$

Mit einer vollelektronischen Messanlage werden die aufgebrachte Auslenkkraft sowie die Verschiebungen $v_{o}$ und $v_{u}$ kontinuierlich aufgezeichnet.

\subsubsection{Versuchsgegenstand}

\subsubsection{Sandwichelemente}

Die Untersuchungen wurden an Sandwichelementen verschiedener Hersteller durchgeführt [9] bis [12]. Es wurden sowohl Dachelemente mit einseitig trapezprofi- 
lierter Deckschicht als auch Wandelemente mit beidseitig quasiebenen Deckschichten geprüft.

Die Deckschichten der Elemente bestanden in allen Fällen aus Stahlblech mit Nennblechdicken zwischen $0,40 \mathrm{~mm}$ und $0,75 \mathrm{~mm}$, die Kernschichten mit Dicken zwischen $40 \mathrm{~mm}$ und $80 \mathrm{~mm}$ aus Polyurethan-Hartschaum oder Mineralfaserplatten.

Eine Zusammenstellung der geprüften Sandwichelemente ist Bild 3.4 bis Bild $3.8 \mathrm{zu}$ entnehmen:

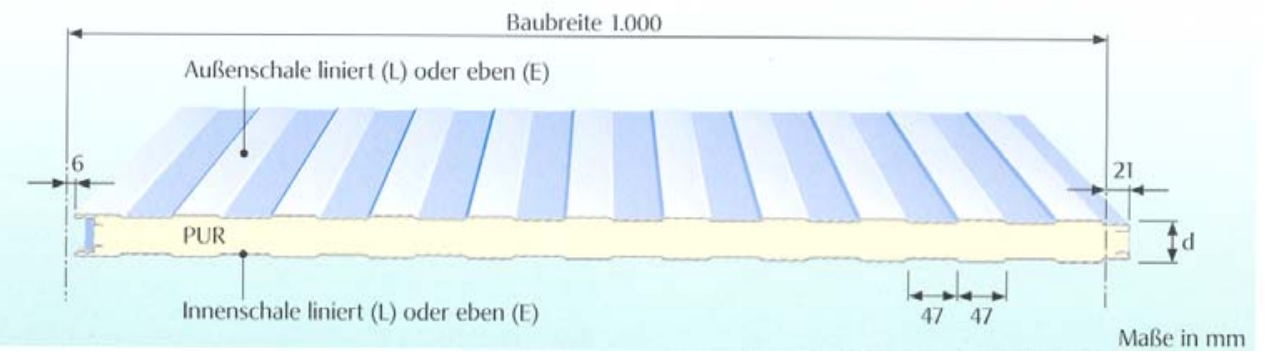

Bild 3.4: Sandwichelement HOESCH isowand LL mit Kernschicht aus Polyurethan [12]

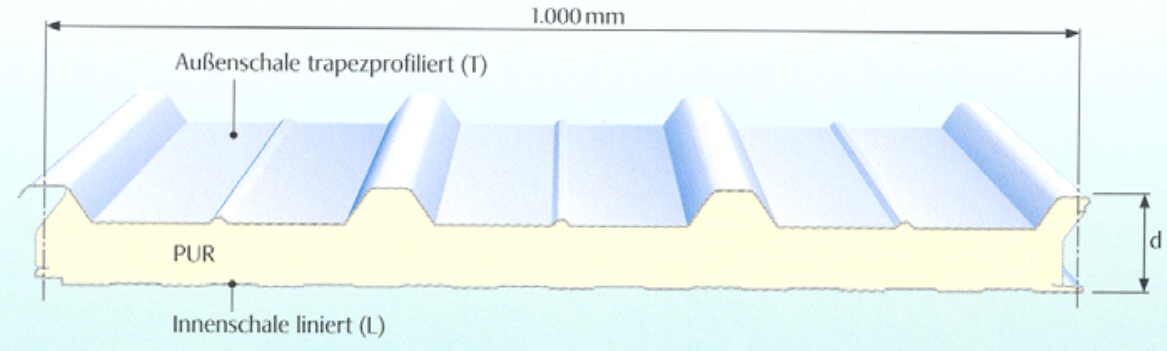

Bild 3.5: Sandwichelement HOESCH isodach TL mit Kernschicht aus Polyurethan [12]

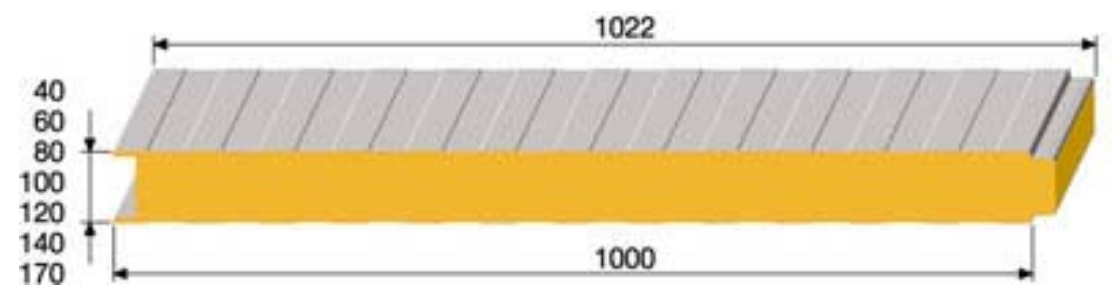

Bild 3.6: Sandwichelement Salzgitter SIP W SB mit Kernschicht aus Polyurethan [10]

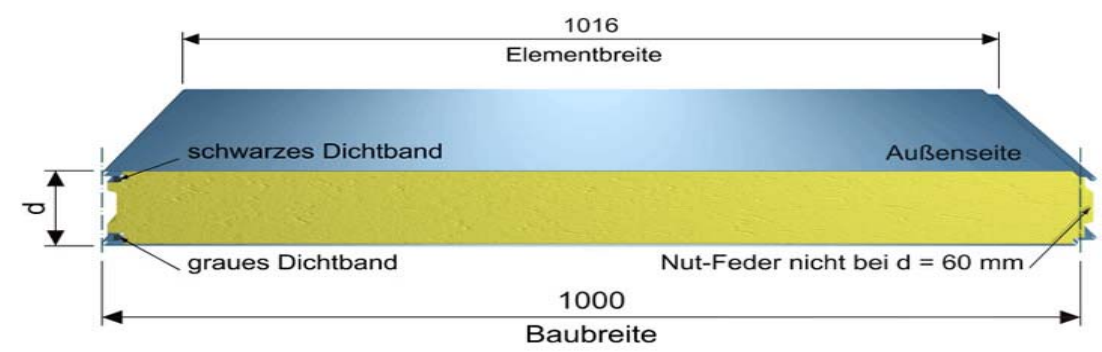

Bild 3.7: Sandwichelement HOESCH isorock mit Kernschicht aus Mineralfaserplatten [9]

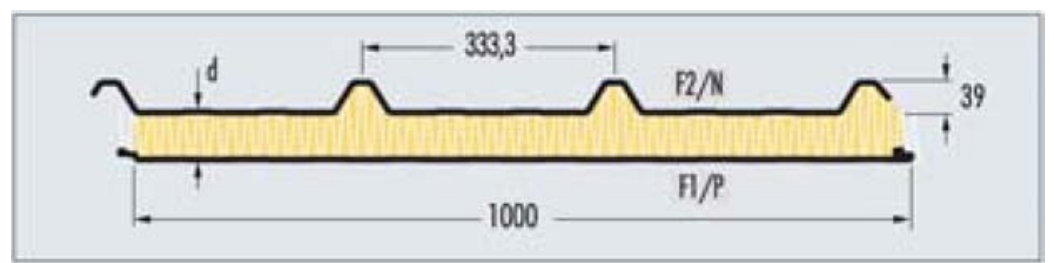

Bild 3.8: Sandwichelement Arcelor Ondafibre $3005 T$ mit Kernschicht aus Mineralfaserplatten [11] 


\subsubsection{Pfettenprofile}

Zum unmittelbaren Vergleich mit den Untersuchungen nach [35] wurden die Versuche mit warmgewalzten I-Trägern des Typs IPE160 aus S235 nach DIN10025-2 durchgeführt. Aufgrund des zunehmenden Einsatzes von kaltgewalzten Profilen im Leichtbau wurden ergänzende Versuche mit Z-Profilen des Typs Z140-65 in Blechdicke $t_{N}=3,0 \mathrm{~mm}$ durchgeführt. Die untersuchten Profile sind in Bild 3.9 dargestellt:

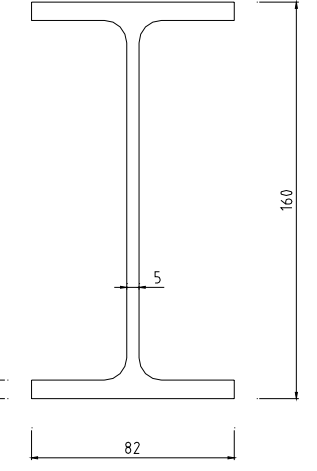

IPE160

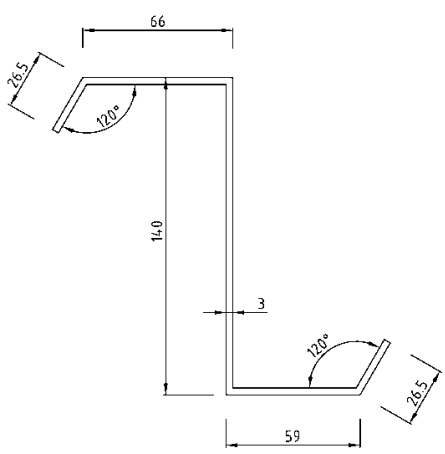

Z140-65

Bild 3.9: untersuchte Pfettenprofile

\subsubsection{Verbindungsmittel}

Die Verschraubung der Sandwichelemente mit den Pfettenprofilen erfolgte entsprechend den Anforderungen aus [9] bis [12] mit gewindefurchenden Schrauben aus nichtrostendem Stahl nach [13] mit aufvulkanisierter EPDM-Dichtung. Der Durchmesser der Dichtscheiben variierte je nach Versuchsreihe zwischen 16 und $22 \mathrm{~mm}$. Die gegenseitige Verschraubung der Elemente im Längsstoß erfolgte in allen Fällen mit Bohrschrauben nach [14] im Abstand von 500 mm. Die eingesetzten Verbindungsmittel sind in Bild 3.10 dargestellt.

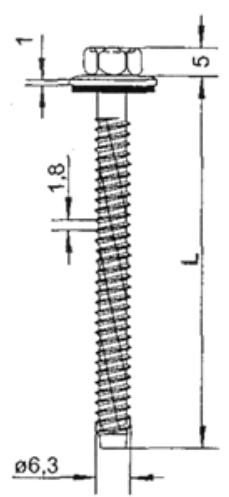

gewindefurchende Schraube Typ EJOT-JZ3-6,3xL

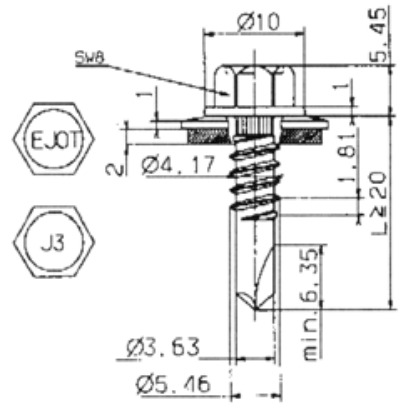

Bohrschraube

Typ EJOT-JT3-2H-5,5x25

\section{Bild 3.10: Verbindungsmittel}

Entsprechend den Anforderungen aus [9] bis [12] wurden die Sandwichelemente in allen Versuchen mit der jeweiligen Mindestanzahl von 2 Schrauben pro Element befestigt. Dies entspricht der im Bauwerk am häufigsten ausgeführten Befestigungsart 
und stellt hinsichtlich der stabilisierenden Wirkung gleichzeitig den ungünstigsten Fall dar.

Die Befestigung der Dachelemente erfolgte in allen Fällen entsprechend Bild 3.11 als direkte Durchschraubung durch beide Deckschichten, Typ TiefsickenBefestigung Minimum nach [12].
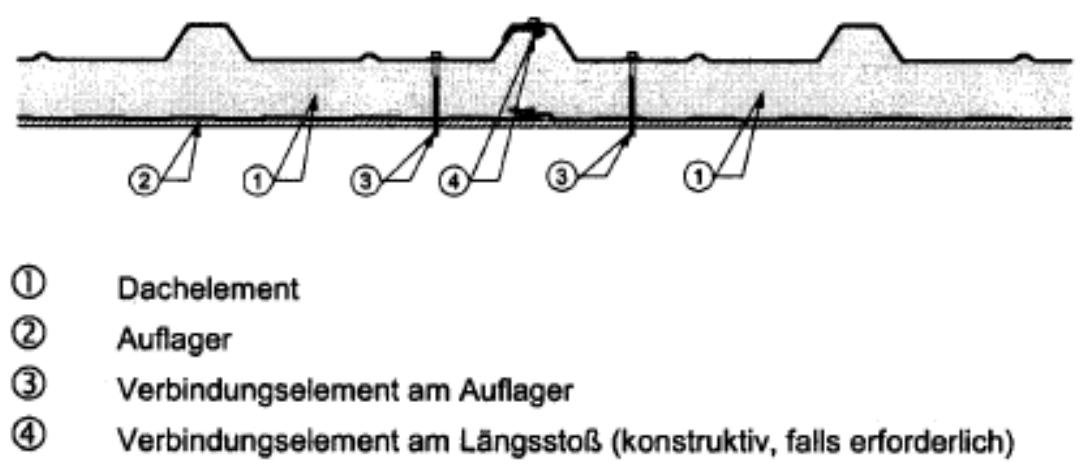

Bild 3.11: Befestigungsart Tiefsicken-Befestigung nach [12]

Die Anordnung der Schrauben erfolgte in Kombination mit doppeltsymmetrischen ITrägern entweder als alternierende Befestigung mit einem Querabstand $e=2,0 \mathrm{~b}_{\mathrm{r}}$ (Typ 1 nach Bild 3.12) oder $e=2,5 b_{r}$ (Typ 2 nach Bild 1.13) oder als einseitige Befestigung mit einem Querabstand $e=2,5 b_{r}$ (Typ 3 nach Bild 3.14), wobei $b_{r}$ dem Profilraster der oberen Deckschicht und $b_{e}$ der Elementbreite entspricht. In Kombination mit Z-Profilen erfolgte die Verschraubung entsprechend Typ 3 in die Mitte des an der Elementunterseite anliegenden Schenkels.

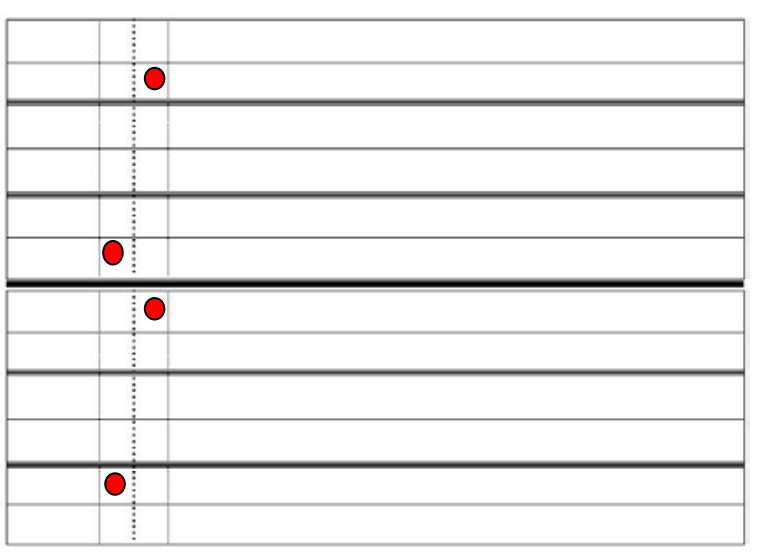

Bild 3.12: Befestigung Typ $1\left(e=2,0 b_{r}\right)$
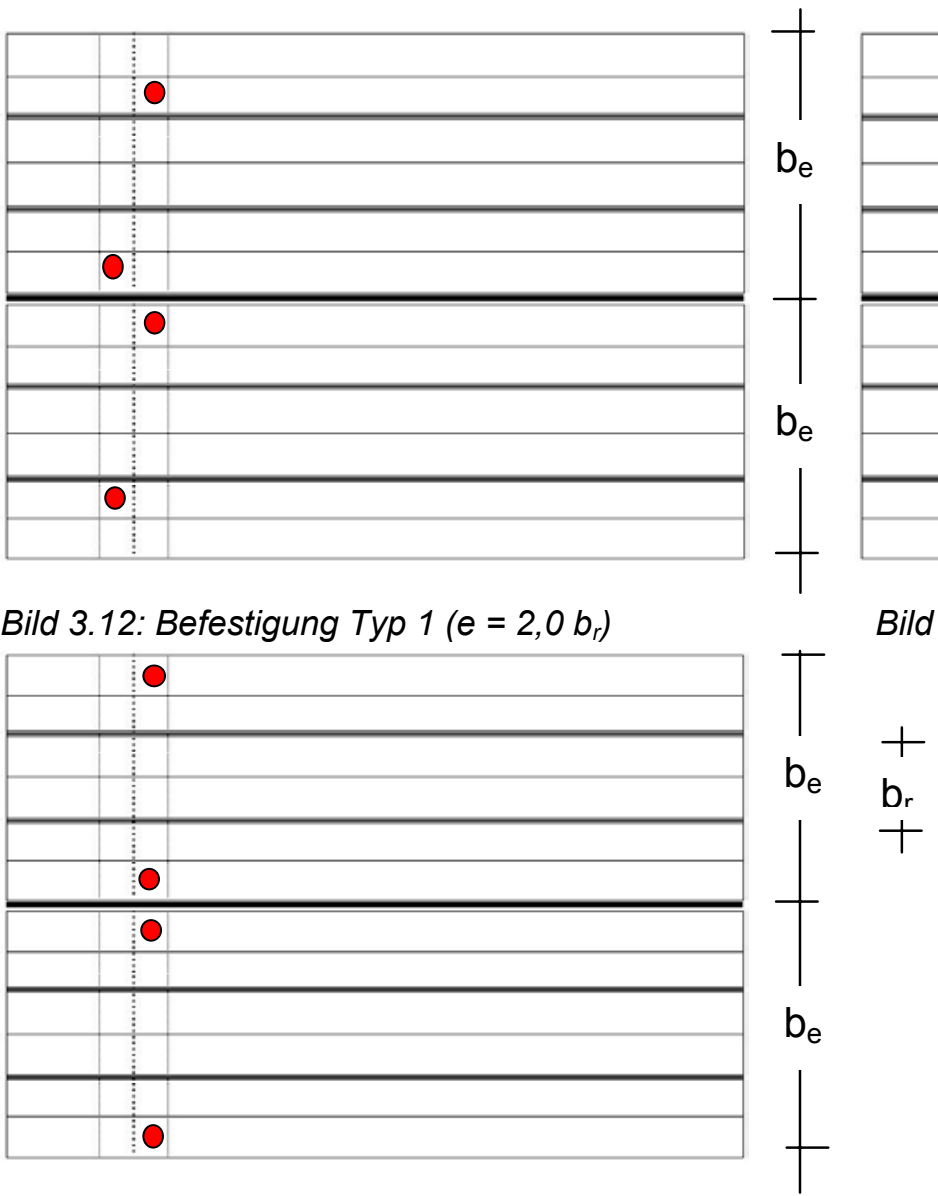

Bild 3.14: Befestigung Typ $3\left(e=2,5 b_{r}\right)$

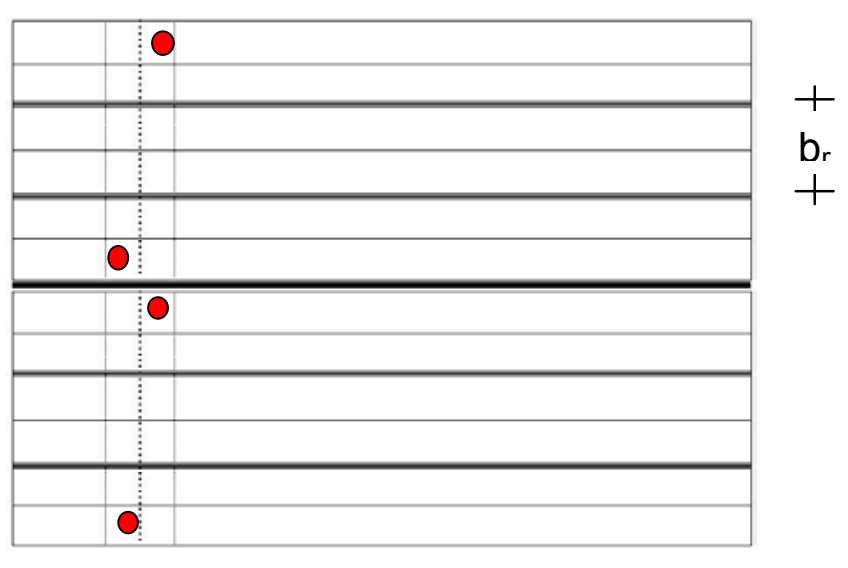

Bild 3.13: Befestigung Typ $2\left(e=2,5 b_{r}\right)$
$+$

$\mathrm{b}_{\mathrm{r}}$

$+$ 


\subsubsection{Zusammenstellung der Versuchsreihen}

Anhang A1 gibt eine Zusammenstellung aller durchgeführten Versuche wieder. Hierbei sind die Sandwichelemente hinsichtlich Typ, Abmessungen und technischer Regel aufgeführt. Typ und Anordnung der Verbindungsmittel sowie die Art der Unterkonstruktion sind ebenfalls mit aufgeführt.

\subsubsection{Versuchsdurchführung}

Nach dem Verschrauben auf der Unterkonstruktion werden die Sandwichelemente über die lastverteilenden Träger mit der Auflast $p_{1}$ flächig vorbelastet. Anschließend wird die drehbar gelagerte Pfette über die mit der Auslenkkraft $F$ belasteten Hebelarme verdreht. Die Auslenkung erfolgt in mehreren Zyklen abwechselnd in positive und negative Drehrichtung, wobei die Amplitude der Auslenkung stetig bis zu einer maximalen Verdrehung $\vartheta \approx 0,1$ rad zunimmt. Nachdem diese maximale Verdrehung sowohl in positiver als auch in negativer Drehrichtung erreicht worden ist, wird die flächige Auflast auf den Wert $\mathrm{p}_{2}$ gesteigert. Die Pfette befindet sich dabei in unausgelenkter Stellung. Anschließend wird die zyklische Verdrehung der Pfette unter der flächigen Auflast $p_{2}$ wiederholt. Zuletzt erfolgt die Pfettenverdrehung unter der Auflast $p_{3}$. Hierbei liegt die Größe der Auflast $p_{3}$ stets im Grenzbereich der Tragfähigkeit des Sandwichelementes, welcher durch Erreichen der zulässigen Knitterspannung definiert ist. Durch dieses Vorgehen lässt sich der Einfluss einer gesteigerten Auflast p auf die Drehbettungswirkung des Sandwichelementes feststellen.

Das zur Auslenkung der Pfette aufgebrachte Torsionsmoment sowie die daraus resultierende Pfettenverdrehung werden kontinuierlich aufgezeichnet und als Momenten-Verdrehungsbeziehung entsprechend Bild 3.15 dargestellt:

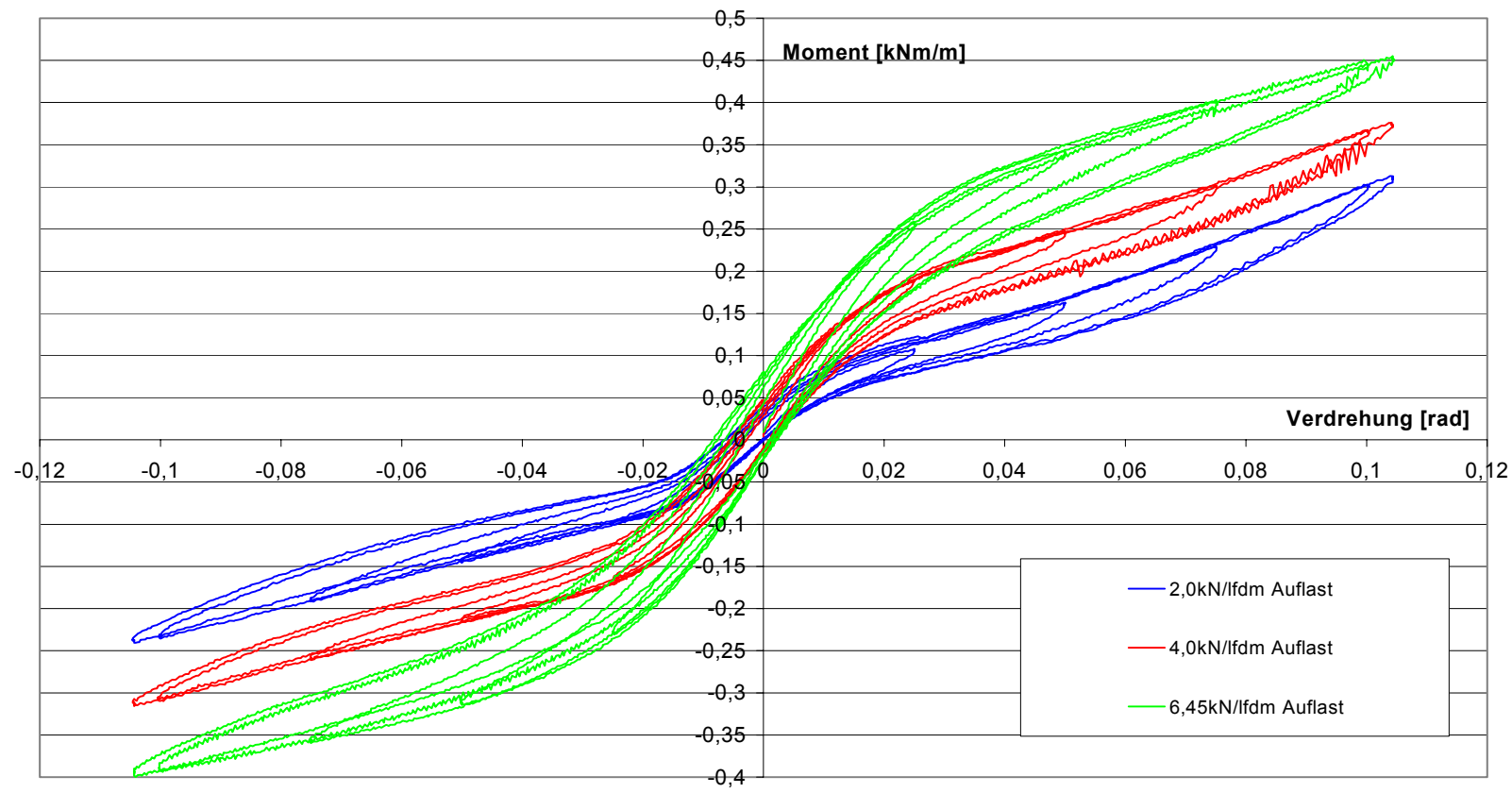

Bild 3.15: exemplarische Momenten-Verdrehungsbeziehung für 3 unterschiedliche Auflasten

Bild 3.15 zeigt, dass die Versuchskurven im Bereich zwischen 0,02 rad $\leq \vartheta \leq 0,02$ rad eine deutlich größere Steigung besitzen als außerhalb dieses Intervalls. Der Einfluss 
von gesteigerten Auflasten wird dadurch deutlich, dass zum Erreichen einer bestimmten Pfettenverdrehung mit steigender Auflast größere Drehmomente notwendig sind. Bereits hier wird deutlich, dass die Auflast einen maßgeblichen Anteil an der Bettungswirkung von Sandwichelementen auf Träger besitzt.

In den folgenden Abschnitten werden die Einflüsse unterschiedlicher Parameter auf die Momenten-Verdrehungsbeziehungen und damit auf die stabilisierende Wirkung eingehend betrachtet.

\subsubsection{Ergebnisse der Drehbettungsversuche}

\subsubsection{Tabellarische Übersicht}

Die Auswahl der Sandwichelemente erfolgte aus der marktüblichen Produktpalette verschiedener Hersteller, um eine möglichst breite Streuung der schaumtechnologischen Kennwerte aus dem Produktionsprozess zu erreichen. Bei der Zusammenstellung der Versuchsreihen wurde darauf geachtet, dass sich die Unterschiede zwischen den einzelnen Versuchen auf jeweils einen Parameter beschränkte. Damit konnte der Einfluss des variierten Parameters durch direkten Vergleich der jeweiligen Versuchsaufzeichnungen bestimmt werden. Die Variation hinsichtlich Elementtyp, Pfettenprofil und Befestigungsart erfolgte entsprechend den Ausführungen aus Abschnitt 3.1.2. Die Größe der Stützweite variierte zwischen 2,5 und 3,5 m.

Tabelle 3.1 gibt eine Zusammenstellung der Parameterkombinationen aller durchgeführten Drehbettungsversuche wieder und verweist insbesondere auf die Diagramme A1 bis A17 des Anhangs A2, wo die aufgezeichneten Momenten-Verdrehungsbeziehungen für jeden Versuch wiedergegeben sind. 


\begin{tabular}{|c|c|c|c|c|c|c|c|c|}
\hline $\begin{array}{c}\text { Versuchs- } \\
\text { bezeichnung }\end{array}$ & $\begin{array}{c}\text { Unter- } \\
\text { konstruktion }\end{array}$ & Elementtyp & $\mathrm{t}_{\mathrm{o}} / \mathrm{t}_{\mathrm{u}}$ & $\mathrm{I}_{\mathrm{s}}$ & $\begin{array}{l}\varnothing \text { Dicht- } \\
\text { scheiben }\end{array}$ & $\begin{array}{l}\text { Verschrau- } \\
\text { bung }\end{array}$ & $\begin{array}{l}\text { Auflasten } \\
\mathrm{p}_{1} / \mathrm{p}_{2} / \mathrm{p}_{3}\end{array}$ & $\begin{array}{l}\text { Dia- } \\
\text { gramm }\end{array}$ \\
\hline & & & {$[\mathrm{mm}]$} & {$[\mathrm{m}]$} & {$[\mathrm{mm}]$} & & {$[\mathrm{kN} / \mathrm{m}]$} & \\
\hline D1/PUR & IPE160 & $\begin{array}{l}\text { HOESCH TL75 } \\
\text { (Bild 3.5) }\end{array}$ & $0,50 / 0,40$ & 3,5 & 16 & Typ 2 & $2,0 / 3,0 / 3,8$ & A1 \\
\hline D2/PUR & IPE160 & $\begin{array}{l}\text { HOESCH TL75 } \\
\text { (Bild 3.5) }\end{array}$ & $0,75 / 0,55$ & 3,5 & 22 & Typ 1 & $2,7 / 4,2 / 5,3$ & A2 \\
\hline D3/PUR & IPE160 & $\begin{array}{l}\text { HOESCH TL95 } \\
\text { (Bild 3.5) }\end{array}$ & $0,75 / 0,55$ & 3,5 & 22 & Typ 1 & $4,1 / 6,4 / 7,9$ & A3 \\
\hline D4/PUR & IPE160 & $\begin{array}{l}\text { HOESCH TL75 } \\
\text { (Bild 3.5) }\end{array}$ & $0,50 / 0,40$ & 2,5 & 16 & Typ 2 & $2,0 / 2,5 / 3,8$ & A4 \\
\hline D5/PUR & IPE160 & $\begin{array}{l}\text { HOESCH TL75 } \\
\text { (Bild 3.5) }\end{array}$ & $0,75 / 0,55$ & 2,5 & 22 & Typ 1 & $2,0 / 4,0 / 6,5$ & A5 \\
\hline D6/PUR & IPE160 & $\begin{array}{l}\text { HOESCH TL95 } \\
\text { (Bild 3.5) }\end{array}$ & $0,75 / 0,55$ & 2,5 & 22 & Typ 1 & $3,0 / 6,0 / 9,2$ & A6 \\
\hline W1/PUR & IPE160 & $\begin{array}{c}\text { Salzgitter SIP } \\
\text { W40 SB (Bild 3.6) }\end{array}$ & $0,60 / 0,50$ & 3,0 & 16 & Typ 2 & $1,5 / 2,5 / 3,2$ & A7 \\
\hline W2/PUR & IPE160 & $\begin{array}{c}\text { Salzgitter SIP } \\
\text { W40 SB (Bild 3.6) }\end{array}$ & $0,60 / 0,50$ & 3,0 & 19 & Typ 2 & $1,5 / 2,5 / 3,2$ & A8 \\
\hline W3/PUR & IPE160 & $\begin{array}{c}\text { Salzgitter SIP } \\
\text { W40 SB (Bild 3.6) }\end{array}$ & $0,60 / 0,50$ & 3,0 & 19 & Typ 3 & $1,5 / 2,5 / 3,2$ & A9 \\
\hline W4/PUR & IPE160 & $\begin{array}{l}\text { HOESCH LL40 } \\
\text { (Bild 3.4) }\end{array}$ & $0,50 / 0,40$ & 3,0 & 16 & Typ 2 & $1,5 / 2,5$ & A10 \\
\hline W5/PUR & IPE160 & $\begin{array}{l}\text { HOESCH LL40 } \\
\text { (Bild 3.4) }\end{array}$ & $0,50 / 0,40$ & 3,0 & 19 & Typ 2 & $1,5 / 2,5 / 2,8$ & A11 \\
\hline W6/PUR & IPE160 & $\begin{array}{c}\text { Salzgitter SIP } \\
\text { w80 SB (Bild 3.6) }\end{array}$ & $0,60 / 0,50$ & 3,0 & 16 & Typ 2 & $2,0 / 5,0 / 5,7$ & $\mathrm{~A} 12$ \\
\hline D1/MW & IPE160 & $\begin{array}{c}\text { Arcelor Ondafibre } \\
\text { 3005T (Bild 3.8) }\end{array}$ & $0,75 / 0,50$ & 2,7 & 22 & Typ 2 & $2,0 / 3,0 / 4,0$ & A13 \\
\hline W2/MW & IPE160 & $\begin{array}{l}\text { HOESCH isorock } \\
\text { LL80 (Bild 3.7) }\end{array}$ & $0,63 / 0,50$ & 2,5 & 19 & Typ 2 & $2,0 / 2,5 / 3,0$ & A14 \\
\hline D7a/PUR & IPE160 & $\begin{array}{l}\text { HOESCH TL95 } \\
\text { (Bild 3.5) }\end{array}$ & $0,75 / 0,55$ & 2,5 & 22 & Typ 1 & $4,0 / 6,0 / 8,0$ & A15 \\
\hline D7b/PUR & IPE160 & $\begin{array}{l}\text { HOESCH TL95 } \\
\text { (Bild 3.5) }\end{array}$ & $0,75 / 0,55$ & 2,5 & 22 & Typ 1 & $4,0 / 6,0 / 8,0$ & A16 \\
\hline DZ/PUR & Z140-65 & $\begin{array}{l}\text { HOESCH TL75 } \\
\text { (Bild 3.5) }\end{array}$ & $0,50 / 0,40$ & 2,5 & 16 & Typ 3 & $2,1 / 2,6 / 3,4$ & A17 \\
\hline
\end{tabular}

$\mathrm{t}_{\mathrm{o}}=$ Dicke der oberen Deckschicht

$t_{u}=$ Dicke der unteren Deckschicht

$\mathrm{I}_{\mathrm{s}}=$ Elementstützweite

Tabelle 3.1: Parameterkombinationen der experimentellen Untersuchungen

Ausführliche Informationen und Bilder zu den jeweiligen Versuchsreihen sowie Beschreibungen weiterführender Untersuchungen sind in den nachfolgenden Abschnitten aufgeführt.

\subsubsection{Sandwichelemente mit Kernschicht aus Polyurethan-Hartschaum}

An Sandwichelementen mit Kernschicht aus Polyurethan-Hartschaum nach [10] und [12] wurden 15 Drehbettungsversuche mit Parameterkombinationen entsprechend Tabelle 3.1 durchgeführt. Mit Ausnahme der Versuche W4/PUR und W6/PUR, bei denen die Sandwichelemente bei der dritten Laststufe infolge Knittern verfrüht versagten, wurden alle Versuche mit jeweils 3 Stufen unterschiedlicher Auflast durchgeführt. Bild 3.16 zeigt den Versuch D2/PUR an Dachelementen TL 75 nach [12] im ausgelenkten Zustand mit der maximalen Pfettenverdrehung $\vartheta \approx 0,1 \mathrm{rad}$. 


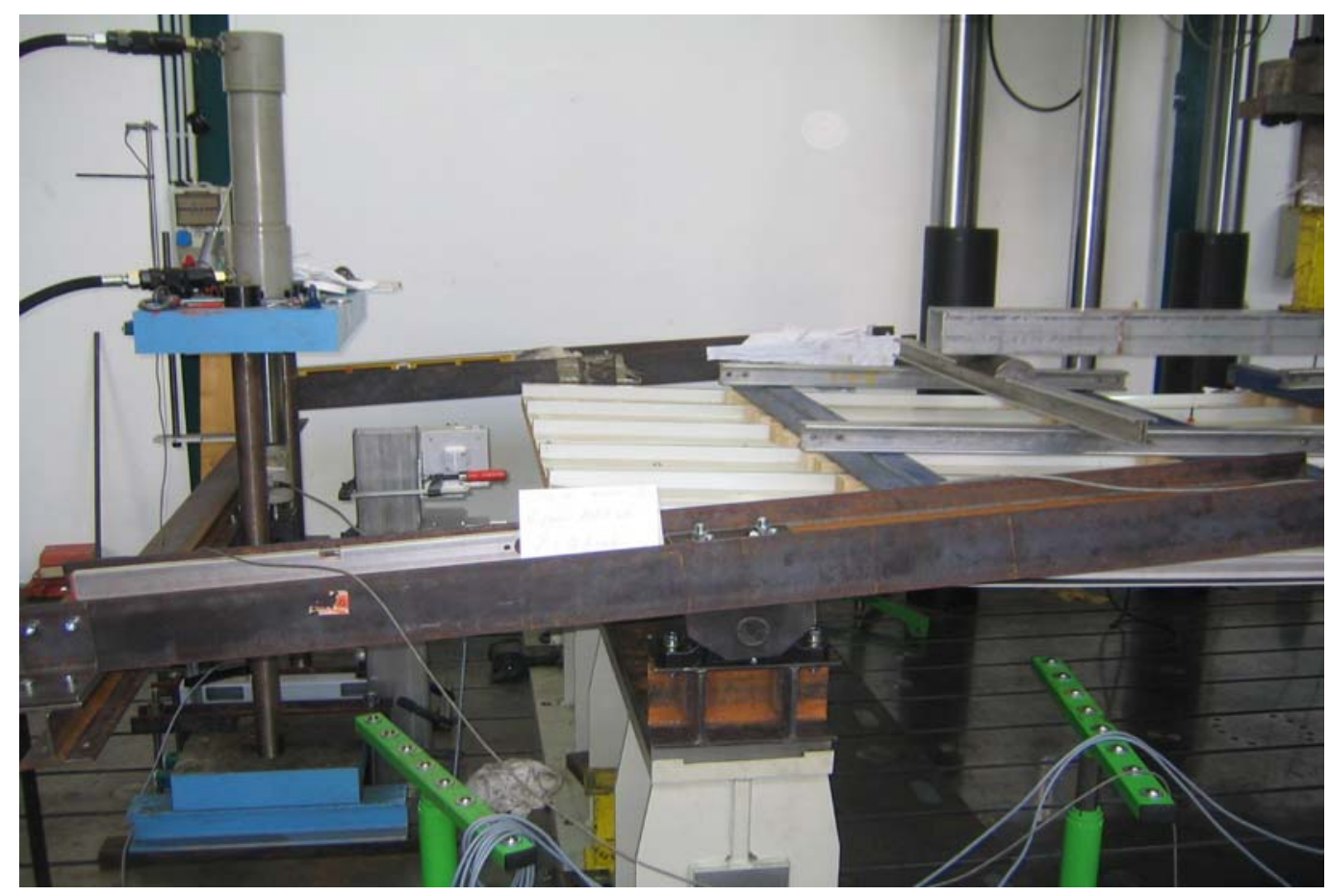

Bild 3.16: Seitenansicht des Versuchs D2/PUR im ausgelenkten Zustand $(\vartheta \approx 0,1 \mathrm{rad})$

Generell traten während der Versuchsdurchführung mit zunehmender Verdrehung lokale Deformationen der oberen Deckschicht im Anschlussbereich infolge Eindrücken der zugbeanspruchten Verbindungsmittel auf. Bei der maximalen Verdrehungen von $\vartheta \approx 0,1$ rad betrugen die Eindrückungen der oberen Deckschicht etwa 1 $2 \mathrm{~mm}$. Infolge der Nachgiebigkeiten im Anschlussbereich entsteht mit zunehmender Verdrehung der Pfette ein Spalt zwischen Pfettenobergurt und unterer Deckschicht des Sandwichelementes. Im Extremfall öffnet sich der Spalt so weit, bis das Sandwichelement nur noch entlang der äußeren Kante des Pfettenobergurts aufliegt (siehe Bild 3.17).

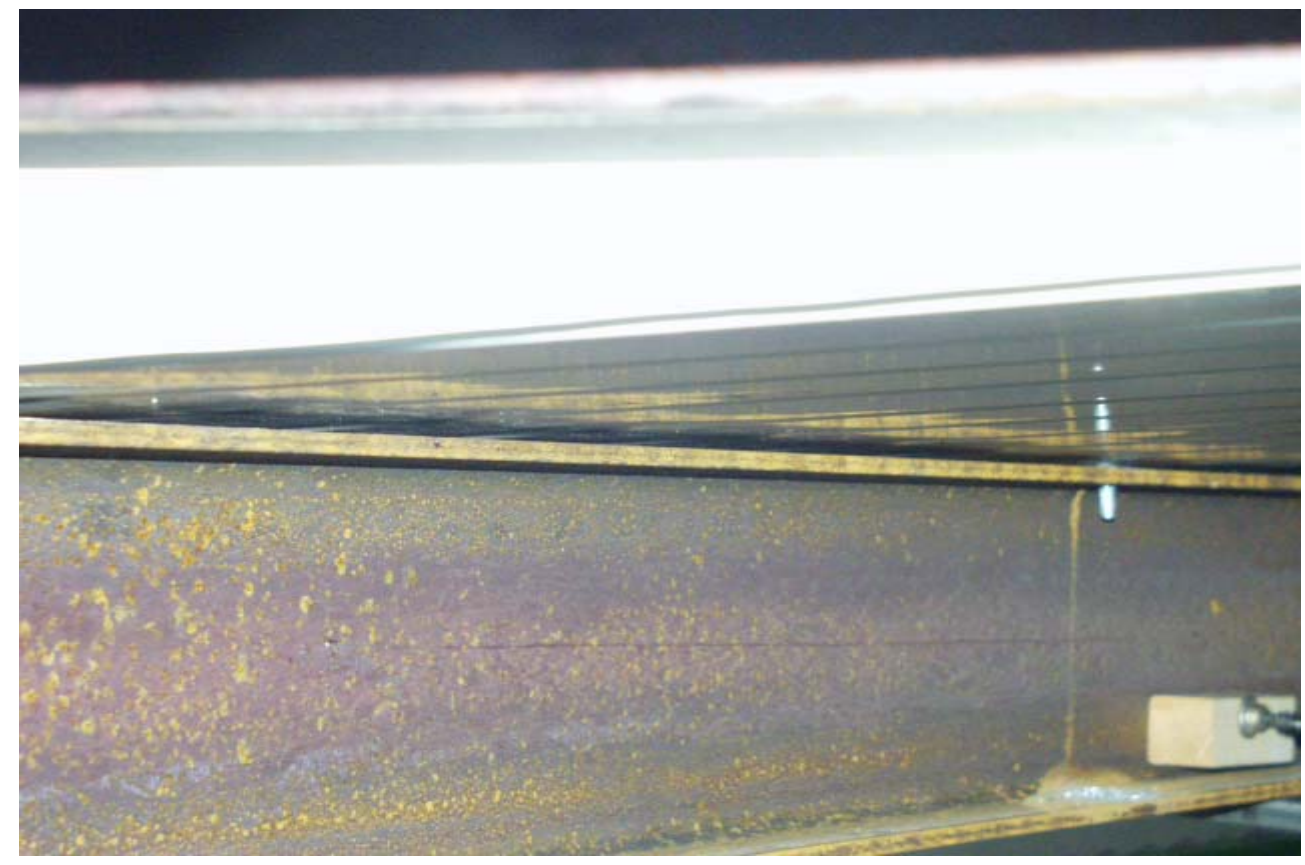

Bild 3.17: Spalt zwischen Pfettenobergurt und Sandwichelement 
Beim Versuch DZ/PUR, der in Kombination mit dem Profil Z-140-65 anstelle des im übrigen verwendeten Profils IPE160 durchgeführt wurde, zeigte sich im Laufe der Versuchsdurchführung mit zunehmender Verdrehung eine deutliche Querschnittsverformung des dünnwandigen Z-Profils. Diese Querschnittsverformung überlagert sich mit Nachgiebigkeiten aus dem Anschlussbereich und beeinflusst die MomentenVerdrehungsbeziehung erheblich.

Von einer Änderung des Versuchsaufbaus zur Verhinderung der Querschnittsverformung oder einer messtechnischen Erfassung der Profilverformung wurde aufgrund des technischen Aufwands abgesehen. Stattdessen wird an dieser Stelle auf Abschnitt 3.2.5.10 verwiesen, wo der Einfluss der Profilverformung sowie einer geänderten Profilgeometrie numerisch untersucht wird.

\subsubsection{Sandwichelemente mit Kernschicht aus Mineralfasern}

Zur Untersuchung der drehbettenden Wirkung von Sandwichelementen mit Kernschicht aus Mineralfasern wurden ergänzende Versuche an Elementen nach [9] und [11] mit Parameterkombinationen entsprechend Tabelle 3.1 durchgeführt. Der Aufbau der Versuche entsprach denen aus Abschnitt 3.1.4.2 und ist beispielhaft für den Versuch D1/MW in Bild 3.18 dargestellt.

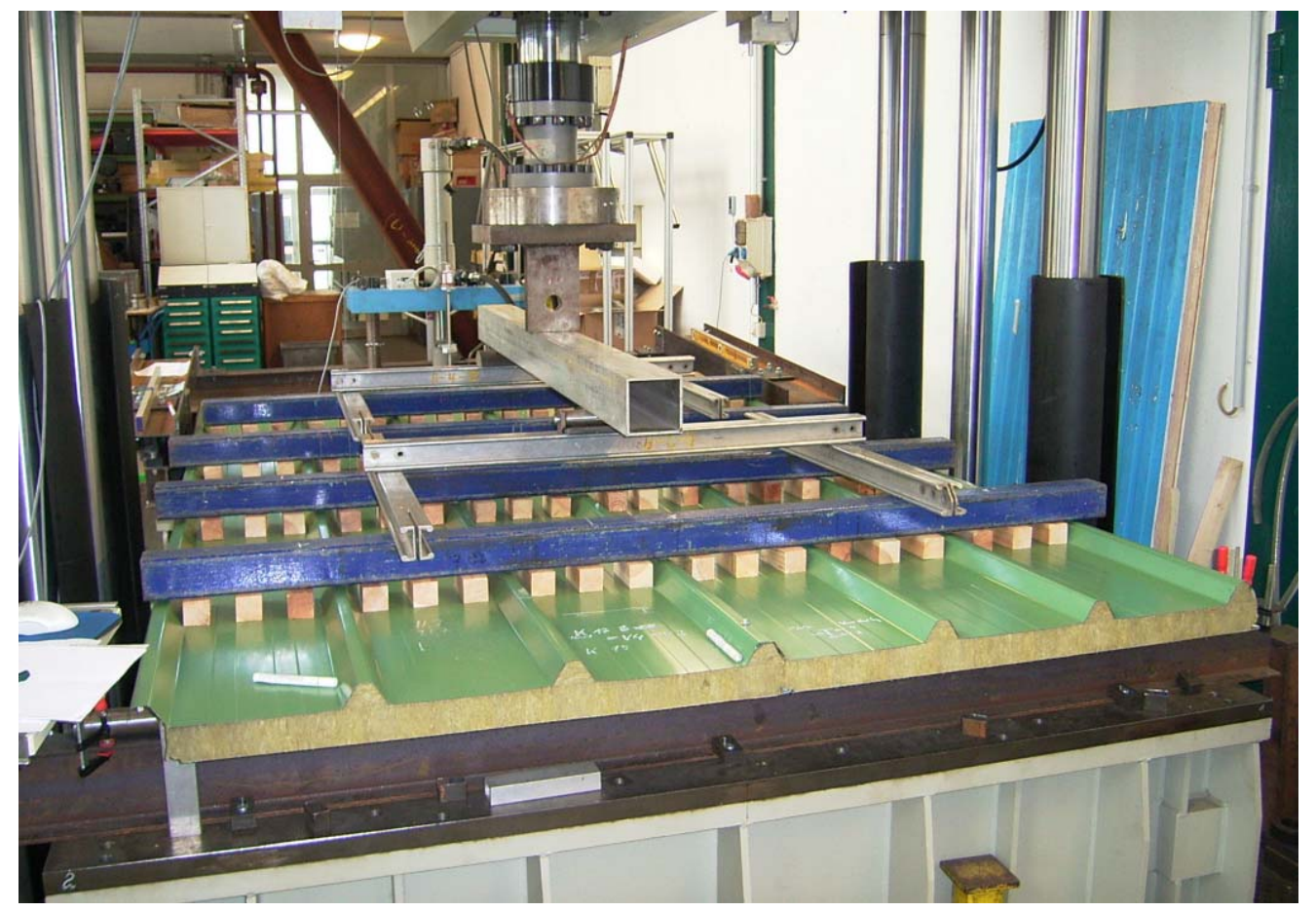

Bild 3.18: Versuch D1/MW mit Mineralfaser-Sandwichelementen Ondafibre 3005T nach [11]

Während der Versuchsdurchführung am Element Ondafibre 3005T nach [11] zeigte sich mit zunehmender Pfettenverdrehung ein Lösen der Verklebung infolge der gegenseitigen Verschiebung (Scherbeanspruchung) zwischen oberer Deckschicht und dem Mineralfaserkern (siehe Bild 3.19). Die Ablösung der Deckschicht erstreckte sich vom freien Ende der Elemente etwa $20 \mathrm{~cm}$ in Richtung der verdrehten Pfette und über beinahe die gesamte Breite der Elemente. 


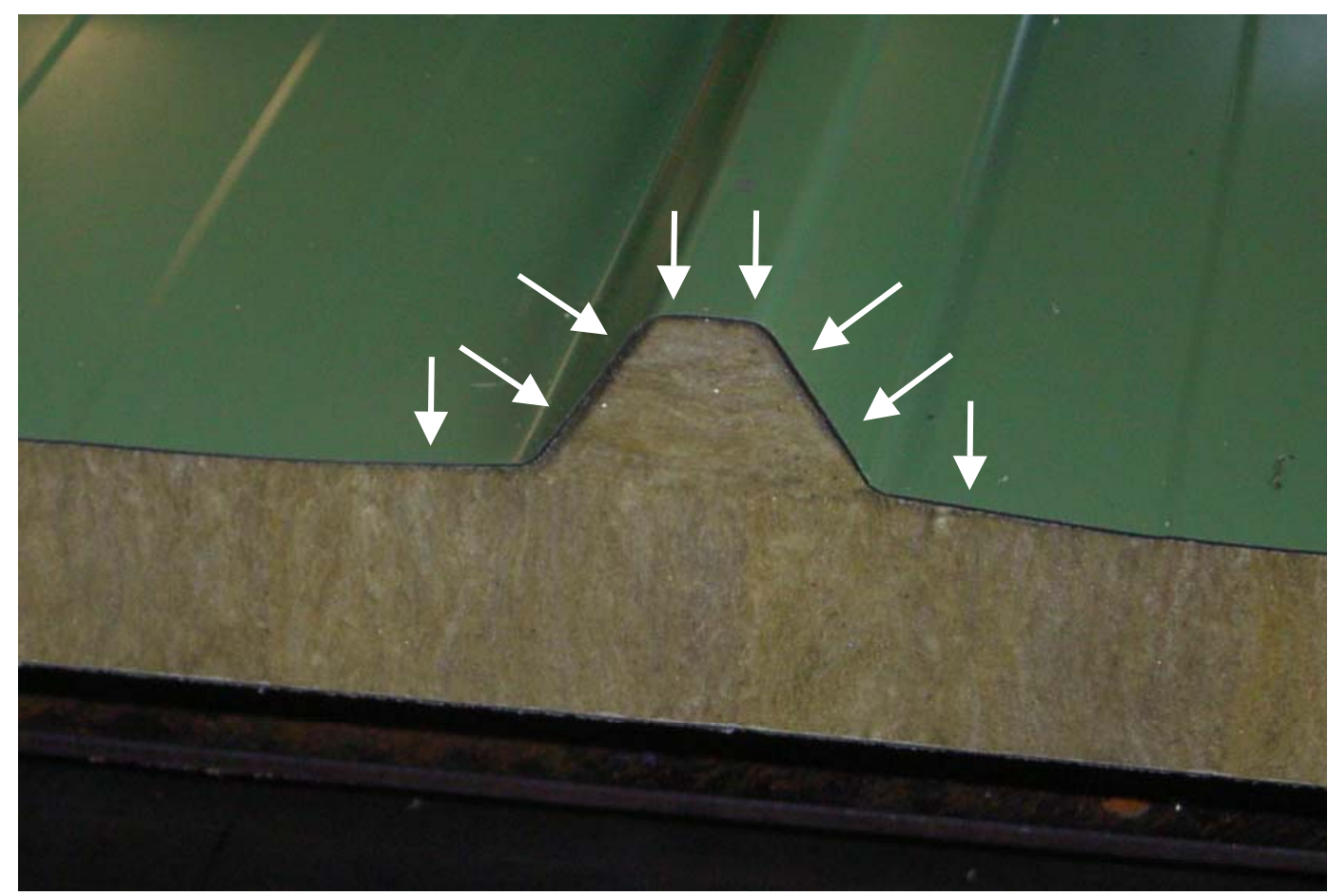

Bild 3.19: abgelöste Deckschicht infolge Scherbeanspruchung beim Versuch D1/MW

Die ermittelte Momenten-Verdrehungsbeziehung des Versuchs W2/MW (Bild 3.20) zeigte die Auffälligkeit, dass ausgehend vom Nullpunkt die Kurven bei den Laststufen $2,5 \mathrm{kN} / \mathrm{m}$ und $3,0 \mathrm{kN} / \mathrm{m}$ eine deutlich geringere Steigung aufweisen als die Kurven bei der Laststufe $2,0 \mathrm{kN} / \mathrm{m}$.

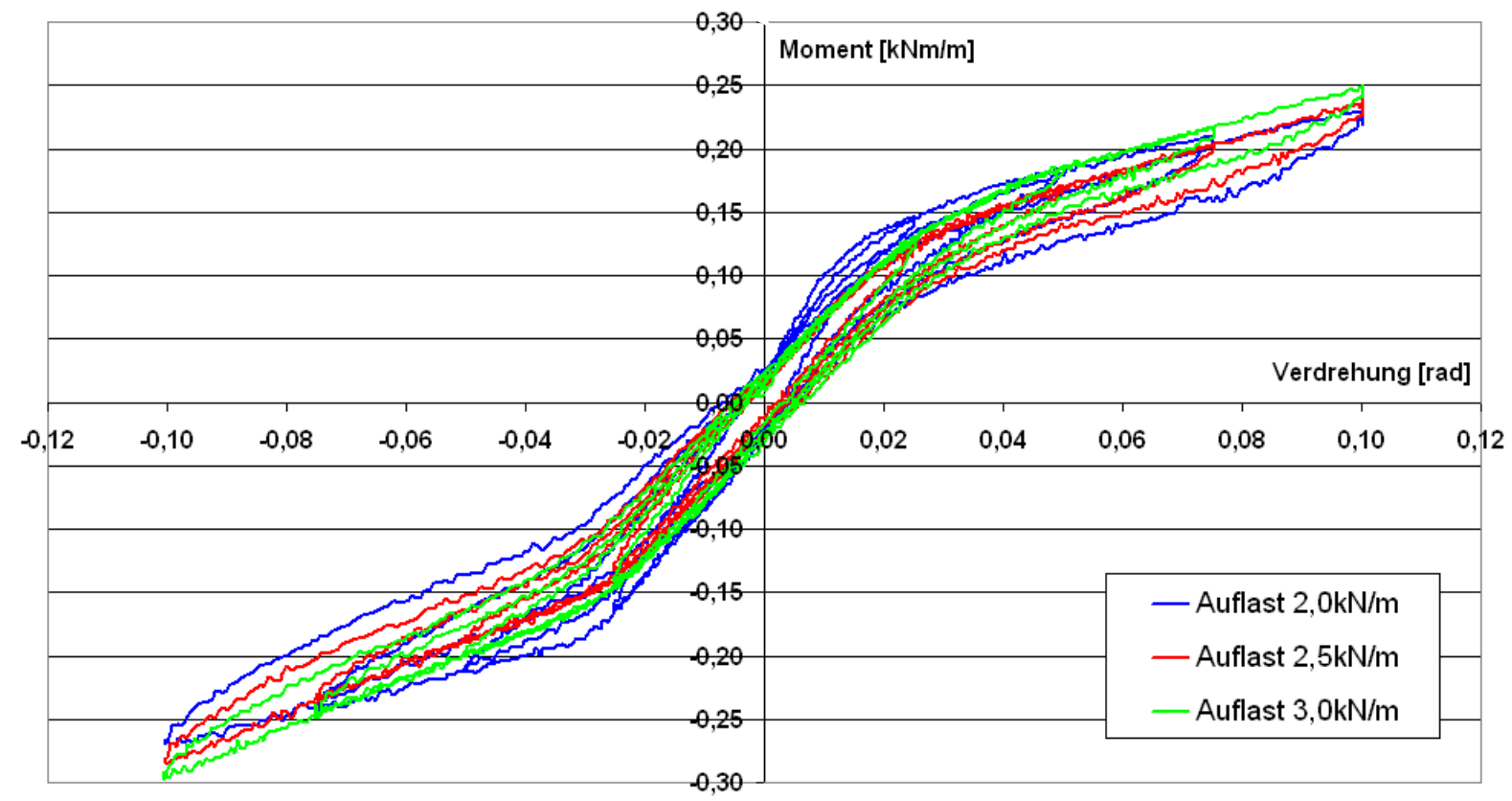

Bild 3.20: Momenten-Verdrehungsbeziehung des Versuchs W2/MW

Die zu Versuchsbeginn vorhandene Systemsteifigkeit wurde im weiteren Versuchsverlauf nicht wieder erreicht. Zum Erreichen einer bestimmten Verdrehung war im weiteren Versuchsverlauf ein kleineres Drehmoment ausreichend. Dieses Verhalten, das ausschließlich bei den Mineralfaser-Sandwichelementen auftrat, ist durch den Aufbau der Mineralfaser-Kernschicht zu begründen. Die in Elementdickenrichtung ausgerichteten Fasern des Kerns erfahren durch Auflast und zusätzlicher Belastung 
infolge Verdrehung der Pfette eine irreversible Schädigung. Diese Schädigung äußert sich in einem verminderten Widerstand gegen ein Eindrehen des Pfettenprofils. Bei der anschließenden Auswertung der Versuchsergebnisse wird dieser Effekt durch Abminderung der bei der ersten Laststufe ermittelten Steifigkeiten berücksichtigt.

\subsubsection{Untersuchungen zum Kriecheinfluss der PUR-Sandwichelemente}

Sandwichelemente mit Kernschicht aus Polyurethan-Hartschaum kriechen unter Dauerbelastung. Da das Kriechen nur durch langzeitige Belastung wie beispielsweise Schnee hervorgerufen wird, sind davon ausschließlich Elemente für den Anwendungsbereich Dach betroffen. Bei der statischen Bemessung von Sandwichelementen wird die kontinuierliche Zunahme der Verformungen unter ständiger Belastung durch den Ansatz eines Kriechmoduls berücksichtigt. Diese Erkenntnis beruht auf zahlreichen Untersuchungen zum Kriechverhalten, zum Beispiel [6], [27], [51].

Inwieweit das Kriechen die drehstabilisierende Wirkung von PUR-Sandwichelementen beeinflusst, wurde durch ergänzende Versuche untersucht. Insbesondere im Auflagerbereich entstehen durch Auflagerpressungen erhebliche Verformungen in der Kernschicht, die sich erst über einen längeren Zeitraum ohne Belastung wieder vollständig zurückbilden. Infolge dieser Verformungen kann wie in Bild 3.21 dargestellt im Anschlussbereich die stützende Wirkung der Kernschicht auf die Verbindungsmittel verloren gehen.

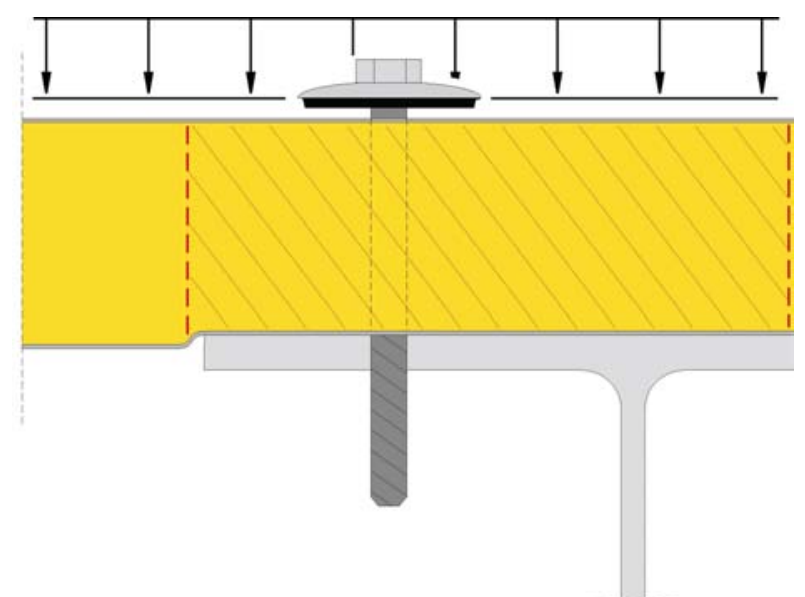

Bild 3.21: auftretende Kriechverformungen im Auflagerbereich (schraffierte Fläche)

Zur Untersuchung dieses Sachverhalts wurden im unmittelbaren Anschluss an einen wie unter Abschnitt 3.1 .3 beschrieben durchgeführten Versuch (D7a/PUR) alle Schrauben der Befestigung auf der Pfette um zwei Umdrehungen gelöst. Aufgrund der etwa 3-4 mm großen Lücke zwischen Schraubenkopf und oberer Sandwichdeckschicht war damit hinsichtlich einer Pfettenverdrehung eine stützende Wirkung der Kernschicht auf die Schraubenköpfe nicht mehr möglich. Nach dem Lösen der Verbindungsmittel wurde der Versuch mit denselben Lastzyklen wie zuvor wiederholt (D7b/PUR). Die Verbindungsmittel dienten hierbei ausschließlich zur Lagesicherung der Elemente auf der Pfette. Durch Vergleich der für beide Versuche aufgezeichneten Momenten-Verdrehungsbeziehungen lässt sich der Kriecheinfluss quantifizieren. 


\subsubsection{Untersuchungen zur Dichtigkeit der Elemente}

Während der Durchführung der Drehbettungsversuche zeigten sich im Anschlussbereich der Elemente deutliche Deformationen, die insbesondere aus der verhältnismäßig großen Pfettenverdrehung (0,1 rad) resultierten. Nach Beendigung der Versuche wurden auf dem oberen Deckblech im Anschlussbereich plastische Verformungen festgestellt (siehe Bild 3.22), die aus dem Eindrücken der Schraubenköpfe resultierten. Der Durchmesser der Schraubentrichter betrug etwa $20 \mathrm{~mm}$ bei einem Durchmesser der Dichtscheibe von $16 \mathrm{~mm}$. Die Tiefe der plastischen Verformung wurde mit $1 \mathrm{~mm}$ gemessen.

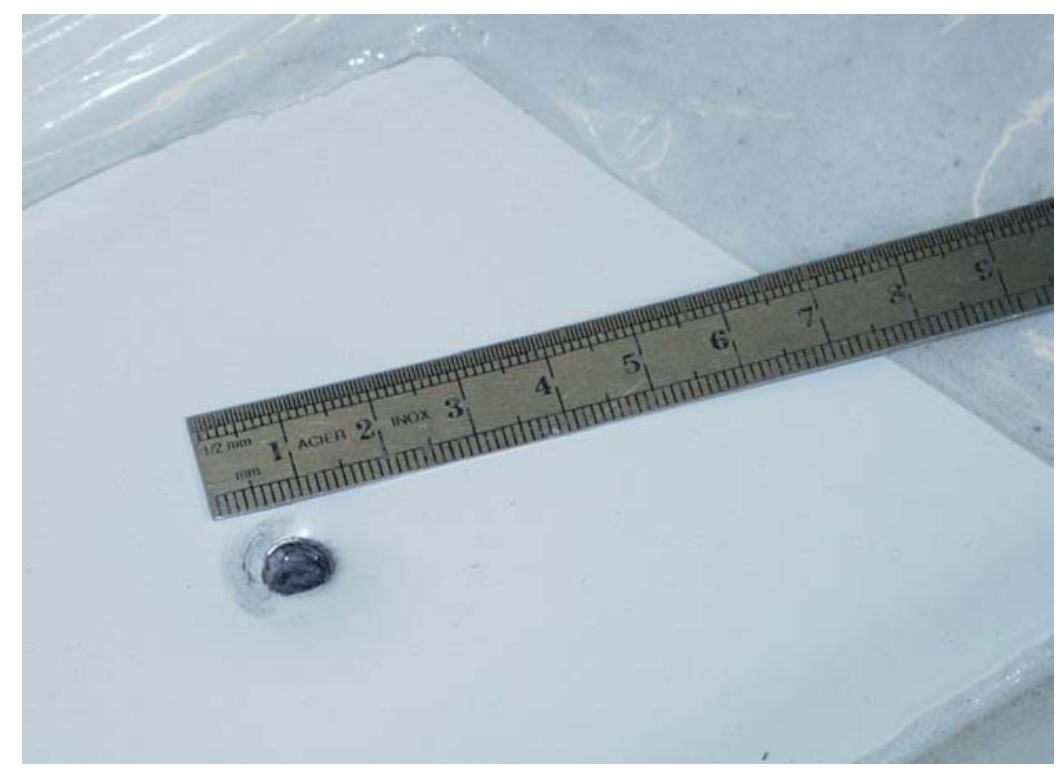

Bild 3.22: plastische Verformungen im Anschlussbereich

Auf der Elementunterseite zeigte sich im Bereich der Durchschraubung eine Langlochbildung. An der Stelle, wo die Sandwichelemente auf der Kante des Pfettenobergurts auflagen, deutete sich eine linienförmige plastische Deformation an.

Zur Prüfung, ob mit den aufgezeigten plastischen Verformungen die Dichtigkeit der Elemente weiterhin gewährleistet ist, wurde beim Versuch D2/PUR nach der eigentlichen Versuchsdurchführung über allen vier Verschraubungen mit der Unterkonstruktion eine $10 \mathrm{~cm}$ hohe Säule gefärbten Wassers aufgebracht (siehe Bild 3.23).

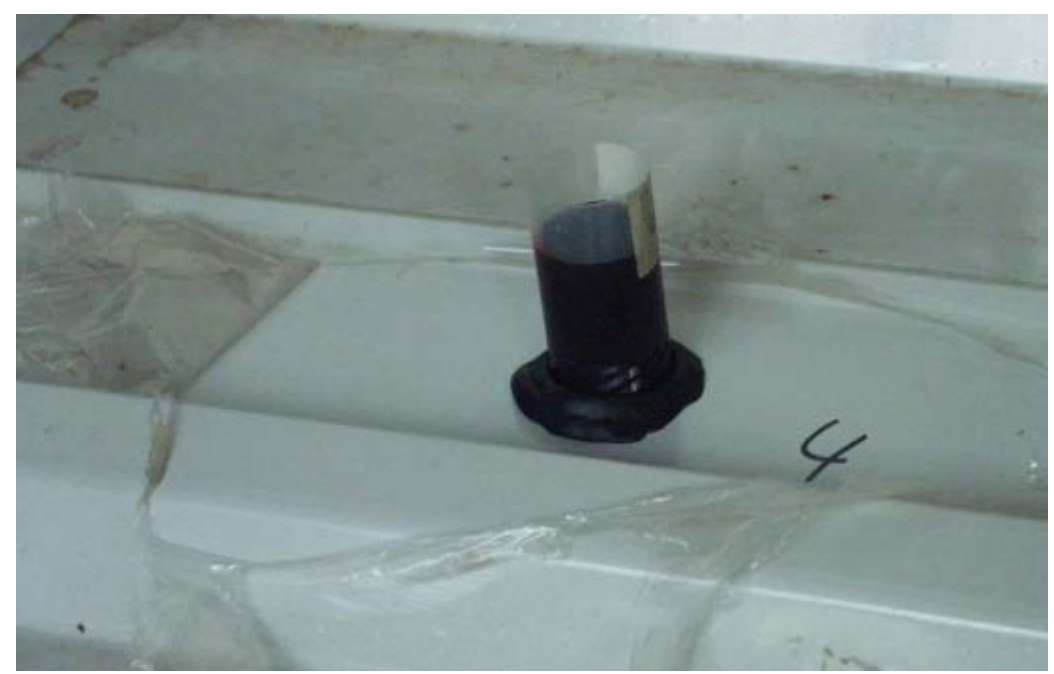

Bild 3.23: Eindringversuch mit $10 \mathrm{~cm}$ Wassersäule über der Verschraubung 
Nach 24 Stunden Einwirkungsdauer wurden die Verschraubungen gelöst und die Elemente zur Beurteilung im Anschlussbereich durchsägt. Der Schnitt offenbarte, dass das Wasser an zwei der vier Schraubenlöcher über die Bohrung in die Kernschicht ein- und teilweise auch durchgedrungen war (siehe Bild 3.24).
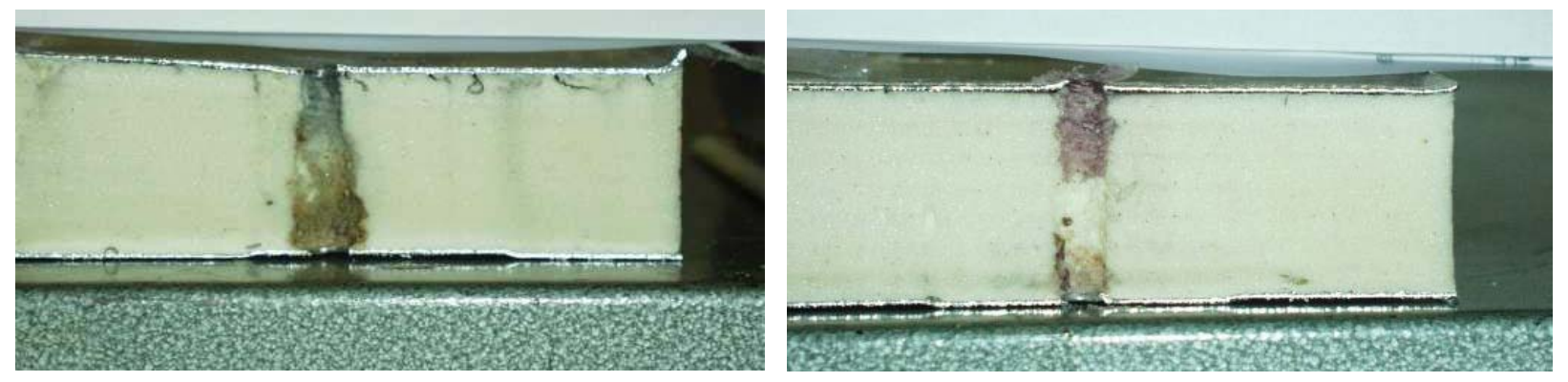

Bild 3.24: Färbungen der Kernschicht infolge eingedrungenem Wasser

Die Dichtigkeit von Sandwichelementen muss stets gewährleistet sein. Deshalb wurde in einem weiteren Versuch an Sandwichelementen des gleichen Typs die Maximalverdrehung auf $\pm 0,08$ rad begrenzt. Bereits während der Versuchsdurchführung zeigten sich deutlich kleinere Verformungen im Anschlussbereich. Plastische Deformationen konnten nach Versuchsende nicht festgestellt werden. Die anschließend durchgeführte Dichtigkeitsprüfung ergab, dass nach 24 Stunden kein Wasser eingedrungen war.

\subsubsection{Bestimmung der Materialkennwerte}

\subsubsection{Allgemeines}

Die aufgezeichneten Momenten-Verdrehungsbeziehungen werden durch zahlreiche Parameter beeinflusst. Im Hinblick auf die Auswertung der MomentenVerdrehungsbeziehungen und die gegebenenfalls durchzuführende Normierung der Ergebnisse sowie die begleitende numerische Untersuchung ist die Kenntnis der vorliegenden Materialkennwerte jedes Elements zwingend notwendig. Zur Bestimmung der jeweiligen Kennwerte wurden die nachfolgend aufgeführten Untersuchungen durchgeführt.

\subsubsection{Mechanische Eigenschaften der Deckschichten}

An jedem geprüften Elementtyp wurden nach Versuchsdurchführung aus den gering beanspruchten Bereichen von oberer und unterer Deckschicht Proben für Zugversuche nach DIN EN 10002-1 ausgearbeitet. Die Durchführung der Zugversuche erfolgte auf einer Universalprüfmaschine der Versuchsanstalt für Stahl, Holz und Steine der Universität Karlsruhe (TH). Für die Bestimmung der Streckgrenze $R_{e H} / R_{p 0,2}$ und der Zugfestigkeit $R_{m}$ wurden die ermittelten Kernblechdicken zugrunde gelegt. Die Ergebnisse sind in Tabelle A3.1 des Anhangs A3 aufgeführt.

Dabei wurde an vier Proben festgestellt, dass die ausgeprägte Streckgrenze $R_{e h}$ größer ist als die Zugfestigkeit $R_{m}$. Dieses Phänomen tritt bei Stählen nach DIN EN 10326 mit zusätzlicher organischer Beschichtung insbesondere bei geringen Blechdicken häufig auf und wird auch durch den Entwurf zu DIN EN ISO 6892 behandelt. Ursache ist vermutlich ein Alterungseffekt, der infolge der Temperatur von ca. $200^{\circ} \mathrm{C}$ während des Coil-Coating-Prozesses und dem zeitgleich erfolgenden Recken des Bandes um $1 \%$ bis $3 \%$ auftritt [22]. 


\subsubsection{Mechanische Eigenschaften der Kernschichten}

Die mechanischen Eigenschaften der Kernschicht aus Polyurethan-Hartschaum werden maßgeblich durch die Schaumrezeptur, den Herstellprozess sowie die Rohdichte des Schaums beeinflusst. Bei Sandwichelementen mit Kernschicht aus Mineralfasern werden die mechanischen Eigenschaften durch die Qualität der in der Regel vorkonfektionierten Mineralfaserplatten bestimmt.

Nach der Durchführung der Drehbettungsversuche wurden aus den gering beanspruchten Bereichen der Elemente Probekörper der Größe 100 mm x $100 \mathrm{~mm}$ x Elementdicke ausgearbeitet und entsprechend DIN EN ISO 291 für 24 Stunden unter Normalklima konditioniert. Bei der Ausarbeitung wurde darauf geachtet, dass die Proben über die gesamte Elementbreite verteilt waren.

Die Bestimmung der Rohdichte $\rho$ erfolgte an jeweils drei Proben entsprechend DIN EN 1602 als Quotient aus der Masse und dem Volumen der Probekörper.

Zur Bestimmung der Zugfestigkeit mit Deckschicht $\sigma_{z}$ nach DIN EN 1607 wurden jeweils drei Probekörper zum Zweck der Krafteinleitung mit Aluminiumplatten verklebt. Die Verklebung erfolgte mit einem 2-Komponentenkleber. Die Zugprüfungen erfolgten auf einer Universalprüfmaschine der Versuchsanstalt für Stahl, Holz und Steine der Universität Karlsruhe (TH), wobei die Verformungen in Dickenrichtung mittels zweier induktiver Wegaufnehmer gemessen wurde. Aus dem linearen Anstieg des ermittelten Last-Verformungsdiagramms wurde der Zugmodul $\mathrm{E}_{Z}$ bestimmt.

Die Druckfestigkeit $\sigma_{D}$ wurde durch Druckversuche nach DIN EN 826 bei $10 \%$ Stauchung bestimmt. Aus dem linearen Anstieg des ermittelten LastVerformungsdiagramms wurde der Druckmodul $E_{D}$ bestimmt.

Die Berechnung des Elastizitätsmoduls $\mathrm{E}_{S}$ erfolgte entsprechend den Bestimmungen aus [9] bis [12] als Mittelwert aus Zug- und Druckmodul eines Probenpaares.

Die ermittelten mechanischen Eigenschaften sind in Tabelle A4.1 des Anhangs A4 aufgeführt.

\subsubsection{Versuchsauswertung}

Die aufgezeichneten und im Anhang A2 wiedergegebenen Momenten-Verdrehungsbeziehungen zeigen eine deutlich ausgeprägte nichtlineare Charakteristik. Im Bereich des Nulldurchgangs besitzen die Kurven eine große Steigung (Anfangssteigung), die bis zu einer Verdrehung $|\vartheta| \approx 0,02$ rad bis 0,03 rad vorliegt. Nach Überschreitung dieser Grenzen haben die Momenten-Verdrehungsbeziehungen einen deutlich flacheren Verlauf.

In [35] erfolgte die Auswertung zur Ermittlung der Steifigkeit $\mathbf{c}_{\vartheta, \mathrm{k}}$ der Gesamtkonstruktion mit einem linearen Verfahren bei einer maximalen Verdrehung von $\vartheta=0,1 \mathrm{rad}$. Hierbei wurde aus der Momenten-Verdrehungsbeziehung das der Verdrehung von $\vartheta=0,1$ rad zugrunde liegende Drehmoment $m_{t}$ ermittelt und mit Gleichung (3.2) 


$$
c_{\vartheta, k}=\frac{m_{t}}{\vartheta}=\frac{m_{t}}{0,1}
$$

als Sekantensteifigkeit ausgewertet. Durch Umstellung der Gleichung (2.6) und anschließende Elimination der Anteile aus der Biegeverformung der Sandwichelemente sowie gegebenenfalls der Profilverformung ergibt sich die Anschlusssteifigkeit $c_{\vartheta A, k}$ $\mathrm{Zu}$

$$
c_{\vartheta A, k}=\frac{1}{\frac{1}{c_{\vartheta, k}}-\frac{1}{c_{\vartheta M, k}}-\frac{1}{c_{\vartheta P, k}}}
$$

Das lineare Verfahren nach [35] lässt jedoch die Nichtlinearitäten im MomentenVerdrehungsdiagramm und damit auch den positiven Effekt der vorliegenden großen Anfangssteigung im Hinblick auf die stabilisierende Wirkung unberücksichtigt.

Die Nichtlinearität des Momenten-Verdrehungsdiagrammes hat folgende Ursache (Bild 3.25):

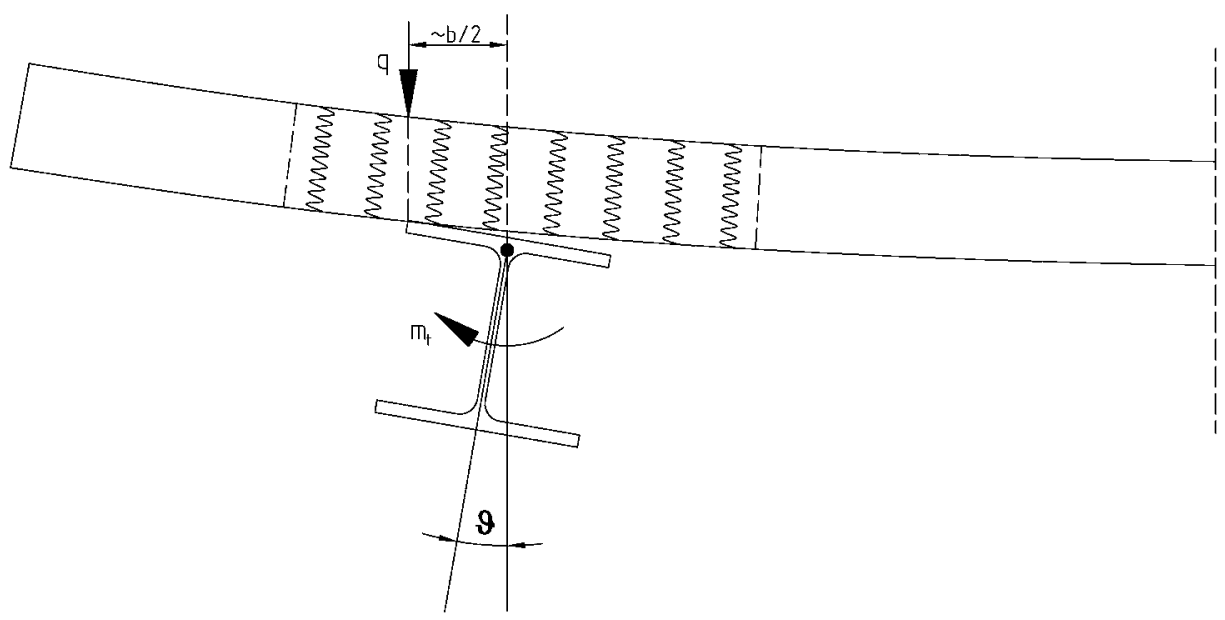

Bild 3.25: mechanisches Modell

In unausgelenkter Stellung der Pfette befindet sich eine ganzflächig verteilte Auflast, deren Resultierende in der Gurtmitte über dem Steg wirkt, auf dem Pfettenobergurt. Mit zunehmender Verdrehung der Pfette entsteht aufgrund von Nachgiebigkeiten im Anschlussbereich ein Spalt zwischen Pfettenobergurt und unterer Deckschicht des Sandwichelements und die Resultierende der Auflast verschiebt sich in Richtung der äußeren Kante des Pfettenobergurts, so dass sie der Drehbewegung entgegenwirkt. Im Extremfall ist der Spalt so weit angewachsen, dass das Sandwichelement theoretisch nur noch entlang der äußeren Kante des Pfettenobergurts aufliegt. Das der aufgebrachten Verdrehung entgegenwirkende, rückstellende Moment erreicht dabei seinen Maximalbetrag

$$
m_{K}=q \cdot \frac{b}{2}
$$

Dieser Zustand ist in [41] auch als Kontaktmoment definiert. Nach Überschreiten des Kontaktmomentes müssen Momentenanteile durch ein Kräftepaar abgetragen wer- 
den, dessen Zugkraft durch die Verbindungsmittel auf das Sandwichelement übertragen wird. Die durch die Zugkraft erzeugten Verformungen mindern die Anschlusssteifigkeit beträchtlich und führen damit zu dem flachen Kurvenverlauf im MomentenVerdrehungsdiagramm.

Zur Auswertung der experimentell ermittelten Momenten-Verdrehungsbeziehungen wird der zuvor dargelegte Sachverhalt sowie das zugehörige mechanische Modell herangezogen. Entsprechend Bild 3.26 werden die beiden Steifigkeitsbereiche der Momenten-Verdrehungsbeziehung durch Geraden mit unterschiedlicher Steigung idealisiert.

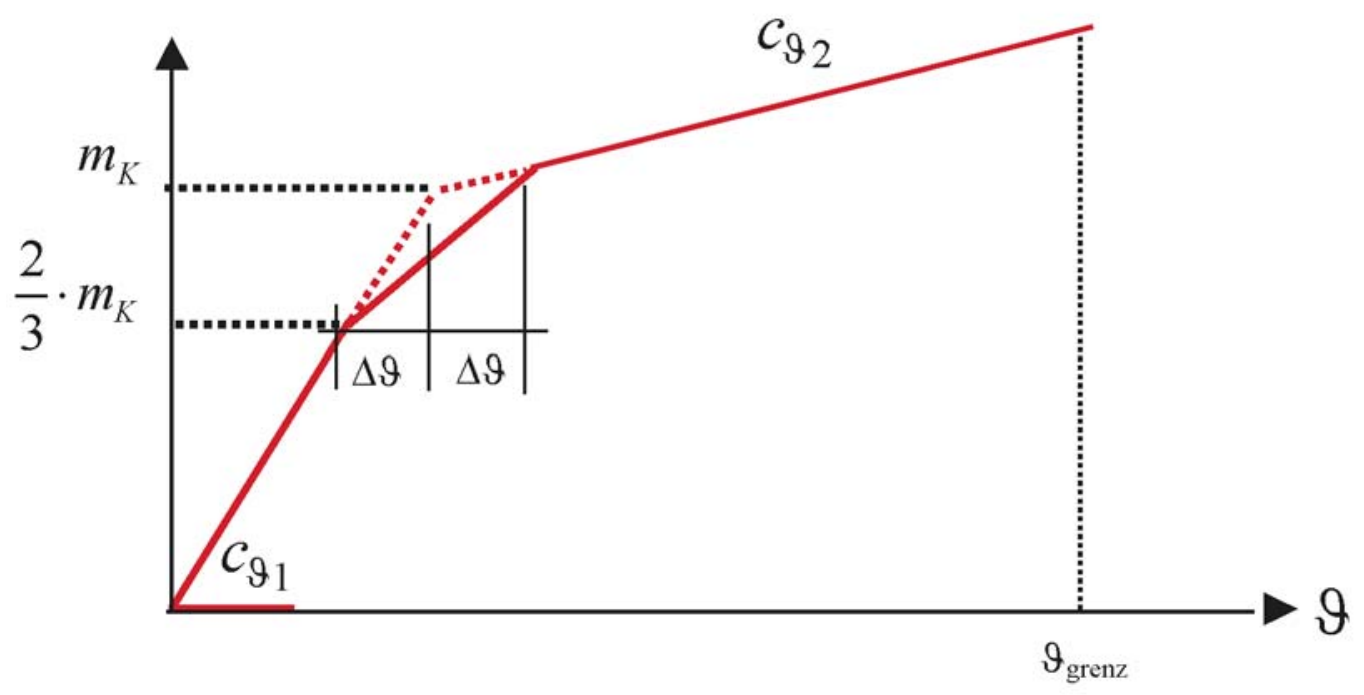

Bild 3.26: Idealisierung der Momenten-Verdrehungsbeziehung

Die große Anfangssteigung im Bereich des Nullpunkts wird hierbei mit $\mathbf{c}_{91}$ und die geringere Steigung nach Überschreiten des Knickpunktes bei der Verdrehung $\vartheta\left(m_{K}\right)$ mit $\mathbf{C}_{92}$ bezeichnet. Bei näherer Betrachtung der Momenten-Verdrehungsbeziehungen im Anhang A2 fällt auf, dass insbesondere bei den Dachelementen der Übergang von $\mathrm{c}_{91} \mathrm{zu}_{\mathrm{C}_{92}}$ nicht diskret, sondern ausgehend von etwa $2 / 3$ des Kontaktmomentes $m_{k}$ stetig erfolgt. Dies liegt unter anderem an der Nachgiebigkeit der Sandwich-Kernschicht, die sich aufgrund der zunehmenden Druckspannungen bei abnehmender Auflagerfläche zunehmend verformt.

Die Idealisierung des Übergangsbereich von $c_{91} z u c_{92}$ im Bereich des nach Gleichung (3.4) ermittelten Kontaktmomentes erfolgt entsprechend Bild 3.26 durch eine Gerade, die sich über einen Bereich von $2 \Delta \vartheta$ erstreckt. Der Wert $\Delta \vartheta$ ergibt sich hierbei als Differenz der Abszissenwerte $\vartheta$ zwischen den Ordinatenwerten $2 / 3 m_{K}$ und $\mathrm{m}_{\mathrm{K}}$.

Die nach diesem Verfahren für jede Laststufe sowohl in positiver als auch in negativer Drehrichtung ermittelten Werte $c_{91}$ und $c_{92}$ sind im folgenden in Tabelle 3.2 für die experimentell untersuchten Dachelemente sowie in Tabelle 3.3 für die experimentell untersuchten Wandelemente aufgeführt. Die dabei angesetzten Geraden zur Idealisierung der beiden Steifigkeitsbereiche sind in den Momenten-Verdrehungsdiagrammen im Anhang A2 eingezeichnet. 


\begin{tabular}{|c|c|c|c|c|c|c|c|}
\hline $\begin{array}{l}\text { Versuchs- } \\
\text { bezeich- } \\
\text { nung }\end{array}$ & $\begin{array}{l}\text { Diagramm- } \\
\text { Nr. im An- } \\
\text { hang A1.2 }\end{array}$ & $\begin{array}{c}\text { Pfettenauflast } \\
\text { inkl. Eigengewicht } \\
\text { des Elements }\end{array}$ & $\mathrm{m}_{\mathrm{k}}$ nach $(3.4)$ & C $_{91 \_p o s}$ & $\mathrm{C}_{91 \_ \text {neg }}$ & 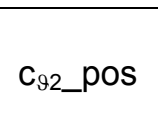 & $c_{92 \_n e g}$ \\
\hline & & {$[\mathrm{kN} / \mathrm{m}]$} & {$[\mathrm{kNm} / \mathrm{m}]$} & {$[\mathrm{kNm} / \mathrm{m}]$} & {$[\mathrm{kNm} / \mathrm{m}]$} & {$[\mathrm{kNm} / \mathrm{m}]$} & {$[\mathrm{kNm} / \mathrm{m}]$} \\
\hline \multirow{3}{*}{ D1/PUR } & \multirow{3}{*}{ A1 } & 2,0 & 0,08 & 6,17 & 6,54 & 1,57 & 1,57 \\
\hline & & 3,0 & 0,12 & 9,14 & 9,22 & 1,41 & 1,33 \\
\hline & & 3,8 & 0,15 & 8,70 & 9,17 & 1,43 & 1,35 \\
\hline \multirow{3}{*}{ D2/PUR } & \multirow{3}{*}{ A2 } & 2,7 & 0,11 & 8,33 & 11,81 & 1,87 & 1,58 \\
\hline & & 4,2 & 0,17 & 8,88 & 10,70 & 2,04 & 1,93 \\
\hline & & 5,3 & 0,22 & 8,77 & 10,42 & 2,00 & 1,79 \\
\hline \multirow{3}{*}{ D3/PUR } & \multirow{3}{*}{ A3 } & 4,1 & 0,17 & 9,66 & 11,43 & 2,18 & 2,62 \\
\hline & & 6,4 & 0,26 & 6,02 & 6,76 & 2,30 & 1,90 \\
\hline & & 7,9 & 0,32 & 10,20 & 10,08 & 2,20 & 2,00 \\
\hline \multirow{3}{*}{ D4/PUR } & \multirow{3}{*}{ A4 } & 2,0 & 0,08 & 9,17 & 8,47 & 1,64 & 1,17 \\
\hline & & 2,5 & 0,10 & 8,47 & 8,65 & 1,70 & 1,20 \\
\hline & & 3,8 & 0,16 & 9,50 & 9,90 & 1,65 & 1,18 \\
\hline \multirow{3}{*}{ D5/PUR } & \multirow{3}{*}{ A5 } & 2,0 & 0,08 & 5,90 & 6,30 & 2,05 & 1,75 \\
\hline & & 4,0 & 0,16 & 8,50 & 9,60 & 2,17 & 1,79 \\
\hline & & 6,5 & 0,26 & 9,50 & 10,90 & 2,22 & 1,59 \\
\hline \multirow{3}{*}{ D6/PUR } & \multirow{3}{*}{ A6 } & 3,0 & 0,12 & 6,70 & 9,10 & 2,08 & 1,53 \\
\hline & & 6,0 & 0,25 & 8,50 & 10,60 & 1,79 & 2,27 \\
\hline & & 9,2 & 0,38 & 9,10 & 10,20 & 1,40 & 2,13 \\
\hline \multirow{3}{*}{ D7a/PUR } & \multirow{3}{*}{ A15 } & 4,0 & 0,16 & 7,17 & 7,50 & 2,01 & 2,53 \\
\hline & & 6,0 & 0,25 & 8,85 & 8,95 & 2,01 & 2,47 \\
\hline & & 8,0 & 0,33 & 9,49 & 9,89 & 1,89 & 2,29 \\
\hline \multirow{3}{*}{ D7b/PUR } & \multirow{3}{*}{ A16 } & 4,0 & 0,16 & 6,19 & 6,44 & 1,01 & 0,73 \\
\hline & & 6,0 & 0,25 & 8,26 & 8,26 & 0,98 & 0,80 \\
\hline & & 8,0 & 0,33 & 8,95 & 9,36 & 1,22 & 0,94 \\
\hline \multirow{3}{*}{ D1/MW } & \multirow{3}{*}{ A13 } & 2,0 & 0,08 & 5,17 & 5,43 & 1,06 & 1,03 \\
\hline & & 3,0 & 0,12 & 5,00 & 5,21 & 1,25 & 1,32 \\
\hline & & 4,0 & 0,16 & 5,30 & 5,56 & 1,09 & 1,16 \\
\hline
\end{tabular}

Tabelle 3.2: Werte $c_{91}$ und $c_{92}$ aus der experimentellen Untersuchung von Dachelementen 


\begin{tabular}{|c|c|c|c|c|c|c|c|}
\hline $\begin{array}{l}\text { Versuchs- } \\
\text { bezeich- } \\
\text { nung }\end{array}$ & $\begin{array}{l}\text { Diagramm- } \\
\text { Nr. im An- } \\
\text { hang A2 }\end{array}$ & $\begin{array}{c}\text { Pfettenauflast } \\
\text { inkl. Eigengewicht } \\
\text { des Elements }\end{array}$ & $m_{k}$ nach (3.4) & c $_{91 \_p o s}$ & $c_{91 \_n e g}$ & $\mathrm{C}_{92 \_ \text {pos }}$ & $C_{92 \_n e g}$ \\
\hline & & {$[\mathrm{kN} / \mathrm{m}]$} & {$[\mathrm{kNm} / \mathrm{m}]$} & {$[\mathrm{kNm} / \mathrm{m}]$} & {$[\mathrm{kNm} / \mathrm{m}]$} & {$[\mathrm{kNm} / \mathrm{m}]$} & {$[\mathrm{kNm} / \mathrm{m}]$} \\
\hline \multirow{3}{*}{ W1/PUR } & \multirow{3}{*}{ A7 } & 1,5 & 0,06 & 5,88 & 6,12 & 0,84 & 1,06 \\
\hline & & 2,5 & 0,10 & 6,93 & 7,37 & 0,99 & 1,02 \\
\hline & & 3,2 & 0,13 & 7,20 & 7,69 & 0,96 & 0,95 \\
\hline \multirow{3}{*}{ W2/PUR } & \multirow{3}{*}{ A8 } & 1,5 & 0,06 & 5,32 & 6,17 & 1,26 & 1,14 \\
\hline & & 2,5 & 0,10 & 6,97 & 6,94 & 1,30 & 1,43 \\
\hline & & 3,2 & 0,13 & 7,43 & 7,43 & 1,16 & 1,36 \\
\hline \multirow{3}{*}{ W3/PUR } & \multirow{3}{*}{ A9 } & 1,5 & 0,06 & 6,46 & 7,75 & 0,59 & 2,05 \\
\hline & & 2,5 & 0,10 & 7,41 & 7,68 & 0,45 & 2,33 \\
\hline & & 3,2 & 0,13 & 7,46 & 7,72 & 0,44 & 2,58 \\
\hline \multirow{2}{*}{ W4/PUR } & \multirow{2}{*}{ A10 } & 1,5 & 0,06 & 4,89 & 5,29 & 1,17 & 1,06 \\
\hline & & 2,5 & 0,10 & 4,90 & 5,08 & 1,40 & 1,40 \\
\hline \multirow{3}{*}{ W5/PUR } & \multirow{3}{*}{ A11 } & 1,5 & 0,06 & 5,36 & 5,58 & 1,23 & 1,09 \\
\hline & & 2,5 & 0,10 & 4,94 & 5,22 & 1,36 & 1,41 \\
\hline & & 2,8 & 0,11 & 4,90 & 5,13 & 1,28 & 1,47 \\
\hline \multirow{3}{*}{ W6/PUR } & \multirow{3}{*}{ A12 } & 2,0 & 0,08 & 6,46 & 7,06 & 1,31 & 0,95 \\
\hline & & 5,0 & 0,21 & 6,10 & 7,63 & 1,32 & 0,94 \\
\hline & & 5,7 & 0,23 & 6,51 & 6,91 & - & - \\
\hline \multirow{3}{*}{ W2/MW } & \multirow{3}{*}{ A14 } & 2,0 & 0,08 & $4,50 *(5,62)$ & $4,40 *(5,22)$ & 1,32 & 1,24 \\
\hline & & 2,5 & 0,10 & 4,55 & 4,15 & 1,42 & 1,88 \\
\hline & & 3,0 & 0,12 & 4,63 & 4,66 & 1,32 & 1,93 \\
\hline
\end{tabular}

*) Werte entsprechend Abschnitt 3.1.4.3 abgemindert

Tabelle 3.3: Werte $c_{91}$ und $c_{92}$ aus der experimentellen Untersuchung von Wandelementen

\subsection{Berechnungen mit der Methode der Finiten Elemente}

\subsubsection{Allgemeines}

Mit der Methode der Finiten Elemente können umfangreiche und kostenintensive Versuchsprogramme auf ein notwendiges Minimum reduziert werden. Das erfordert jedoch die Beurteilung, ob das verwendete Finite-Element-Modell zuverlässige Ergebnisse liefert. Dies geschieht in der Regel durch Vergleich mit experimentellen Untersuchungen, wie sie hier vorliegen, oder analytischen Lösungen.

\subsubsection{Beschreibung des Finite-Element-Modells}

Die Finite-Element-Berechnungen wurden mit dem Programm ANSYS in der Version 8.1 durchgeführt. Umfangreiche Informationen zu diesem Programm und eine Dokumentation der zur Verfügung stehenden Elementbibliothek sind [1] zu entnehmen. 
Das Finite-Element-Modell bildet das Pfettenprofil, das stabilisierende Sandwichelement und die Verbindungsmittel als Ausschnitt eines Dachaufbaus unendlicher Breite ab. Dazu sind die Längsränder des Sandwichelements sowie des Pfettenprofils mit Symmetrierandbedingungen versehen. Analog zu dem unter Abschnitt 3.1.1 beschriebenen Versuchsaufbau wird die Pfettendrehachse im Schnittpunkt von Steg und Obergurt definiert. Die Breite des Dachausschnitts entspricht der Baubreite eines Sandwichelements und variiert damit in Abhängigkeit des untersuchten Elementtyps zwischen $960 \mathrm{~mm}$ und $1150 \mathrm{~mm}$. Bild 3.27 zeigt den schematischen Aufbau des Finite-Element-Modells, bestehend aus einem Pfettenprofil IPE 160 und einem Sandwich-Dachelement mit $60 \mathrm{~mm}$ dicker Kernschicht. Zur besseren Darstellung ist das Sandwichelement in Bild 3.27 mit einer Länge von etwa 1,80 m relativ kurz ausgeführt.

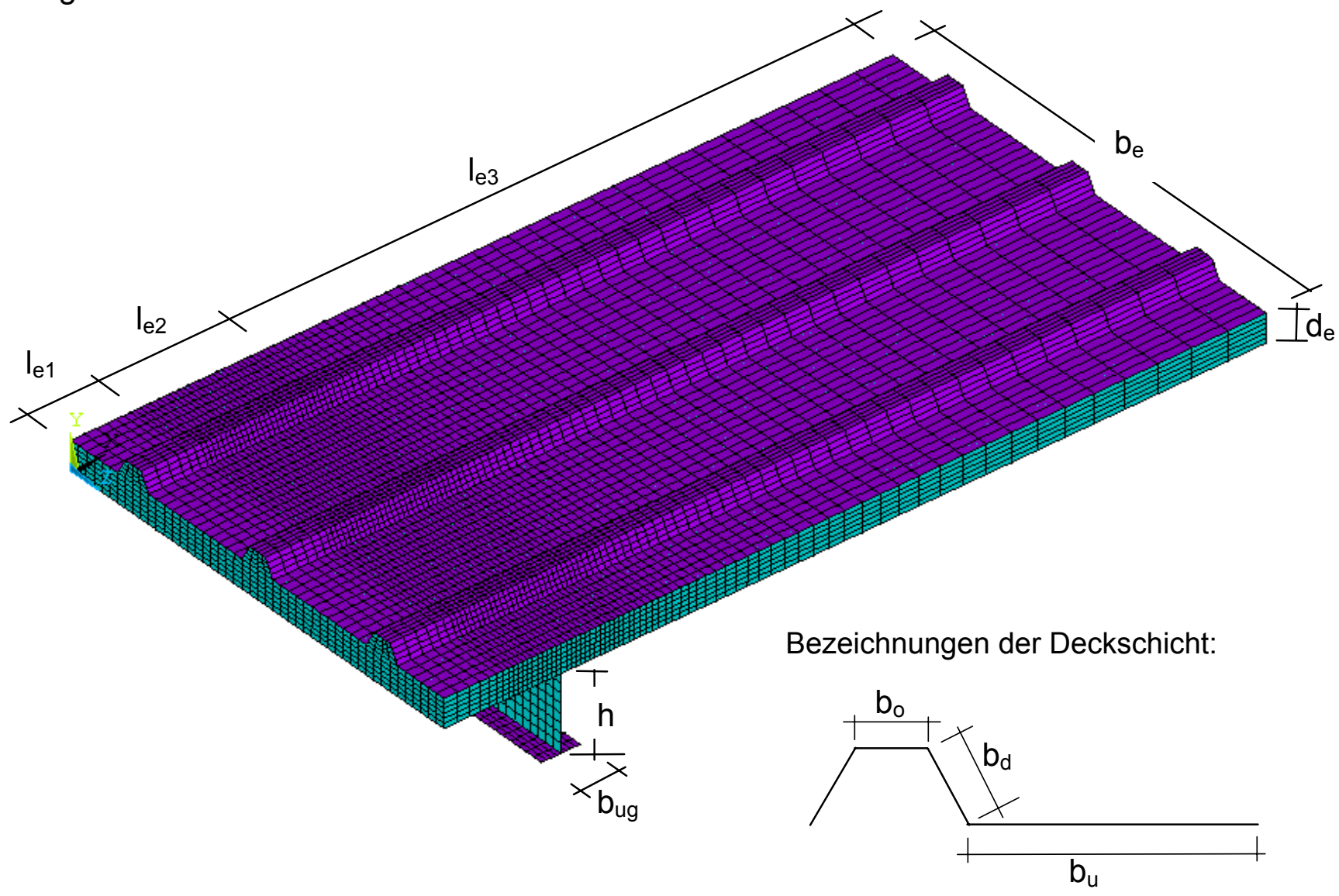

Bild 3.27: Finite-Element-Modell von Sandwichelement und Pfettenprofil

Wie aus Bild 3.27 ersichtlich ist, wurde zur Erfassung der lokalen Deformationen infolge der Pfettenverdrehung und dem damit verbundenen Eindrücken der Dichtscheiben sowohl die Deckschichten als auch die Kernschicht des Sandwichelementes im Bereich des Pfettenprofils mit großer Netzdichte ausgeführt. Die notwendige Netzdichte der Elementeinteilung wurde im Rahmen von Voruntersuchungen bestimmt. Die gewählte Elementeinteilung für den Bereich großer Netzdichte ist für ein Dachelement TL75 in Kombination mit einem Pfettenprofil IPE160 der Tabelle 3.4 zu entnehmen. Für den Fall größerer Abmessungen wurde die Elementanzahl linear erhöht. Zur Vermeidung von Netzstörungen wurden die kreisförmigen Dichtscheiben durch flächengleiche quadratische Elemente modelliert, die unnachgiebig mit den Knoten der oberen Deckschicht verbunden waren. 


\begin{tabular}{|c|c|c|}
\hline Bauteil & Maßbezeichnung & Mindestanzahl der Elemente \\
\hline \multirow{4}{*}{ Sandwichelement } & $\mathrm{d}_{\mathrm{e}}$ & 6 \\
\cline { 2 - 3 } & $\mathrm{l}_{\mathrm{e} 1}$ & 6 \\
\cline { 2 - 3 } & $\mathrm{l}_{\mathrm{e} 2}$ & 26 \\
\cline { 2 - 3 } & $\mathrm{l}_{\mathrm{e} 3}$ & 36 \\
\cline { 2 - 3 } & $\mathrm{b}_{\circ}$ & 4 \\
\cline { 2 - 3 } & $\mathrm{b}_{\mathrm{d}}$ & 4 \\
\cline { 2 - 3 } & $\mathrm{b}_{\mathrm{u}}$ & 12 \\
\hline \multirow{4}{*}{ Pfettenprofil } & $\mathrm{b}_{\mathrm{UG}}$ & 8 \\
\cline { 2 - 3 } & $\mathrm{h}$ & 8 \\
\hline
\end{tabular}

Tabelle 3.4: Elementierung des Finite-Element-Modells nach Bild 3.27

Die Stahldeckschichten des Sandwichelementes, die Dichtscheiben der Verbindungsmittel sowie das Pfettenprofil werden durch Schalenelemente des Typs SHELL181 abgebildet (siehe Bild 3.28). Dieses dreidimensionale Schalenelement wird durch vier Eckknoten mit jeweils drei Verschiebungs- und drei Rotationsfreiheitsgraden definiert. Es besitzt Biege-, Membran- und Schubsteifigkeiten und erfasst auch nichtlineare Materialeigenschaften. Als Werkstoffverhalten wurde in allen Fällen ein bilineares Werkstoffgesetz (linear elastisch, ideal plastisch) angesetzt, wobei nach Überschreiten der Streckgrenze $f_{y}$ Fließen ohne Verfestigung einsetzt. Auf die Modellierung der Dichtungen unter dem Schraubenkopf wurde verzichtet, da bei der anschlagorientierten Verschraubung die EPDM-Dichtung bereits so stark komprimiert wird, dass die in ihr auftretenden Nachgiebigkeiten gegenüber den Nachgiebigkeiten im Sandwichelement vernachlässigt werden können.
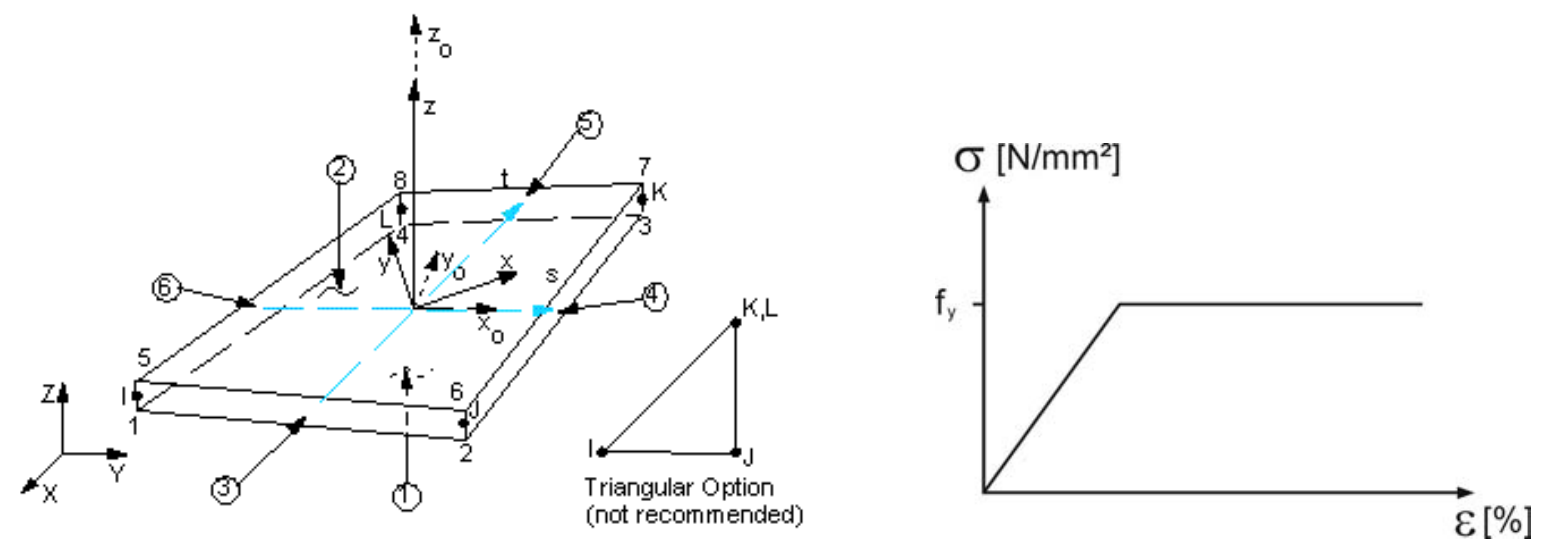

Bild 3.28: Elementtyp SHELL181 [1] mit bilinearem Werkstoffgesetz

Die Sandwichkernschicht wird durch dreidimensionale Volumenelemente des Typs SOLID45 mit acht Eckknoten dargestellt, die jeweils drei Verschiebungsfreiheitsgrade besitzen (siehe Bild 3.29). SOLID45 ist zur Berechnung von Strukturen mit orthotropen Materialeigenschaften geeignet und bietet dem Anwender verschiedene Eingabe- und Berechnungsoptionen, mit denen unter anderem unerwünschte Schubversteifungseffekte vermieden werden können. Zudem können mit diesem Element Berechnungen mit großen Verformungen oder Verzerrungen ausgeführt werden, was beim verwendeten Finite-Element-Modell insbesondere im Bereich der Eindrückungen der Dichtscheiben in das Sandwichelement zum Tragen kommt. Die dabei auftretenden gegenseitigen Verschiebungen benachbarter Knoten des Elements werden durch lineare Ansatzfunktionen approximiert. 

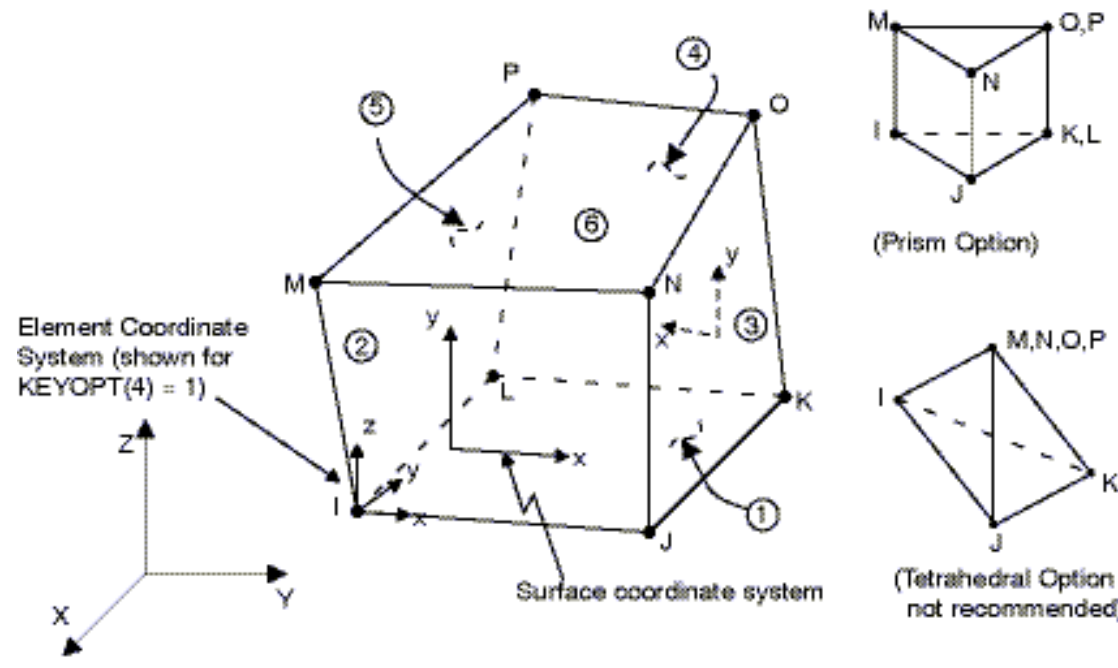

(Prism Option)

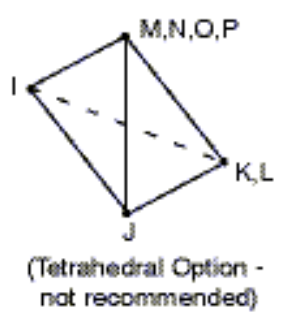

Bild 3.29: Elementtyp SOLID45 [1]

Mit dem Elementtyp SOLID45 können zudem nichtlineare Werkstoffgesetze berücksichtigt werden. Die mechanischen Eigenschaften der Sandwichkernschicht (Elastizitätsmodul, Schubmodul, Querdehnzahl) können unabhängig voneinander für jede Koordinatenrichtung beliebig definiert werden.

Die Befestigung des Sandwichelements auf dem Pfettenprofil wird durch Elemente des Typs LINK8 abgebildet. Dieses dreidimensionale Element verbindet jeweils einen Knoten des Pfettenprofils mit einem Knoten der modellierten Schraubendichtscheibe auf der Sandwichoberseite zur Übertragung der einachsig wirkenden Zugkräfte. Beide Knoten besitzen jeweils drei Freiheitsgrade.

Die Übertragung des Kontaktes zwischen Sandwichelement und Pfettenprofil erfolgt über eine Kontaktfläche zwischen unterer Sandwichdeckschicht und dem Obergurt des Pfettenprofils. Die Kontaktfläche besteht auf Seite des Sandwichelements aus Kontaktelementen des Typs CONTA173 (siehe Bild 3.30) und auf dem Pfettenobergurt aus Kontaktelementen des Typs TARGE170. Diese Elemente sind sogenannte surface-to-surface Kontaktelemente mit jeweils vier Eckknoten, welche zur Beschreibung eines flächigen Kontaktes dienen. Die immer paarweise auftretenden Elemente werden auf bereits modellierten Oberflächen angeordnet und definieren damit mögliche Kontaktzonen. Die Durchdringung dieser einander zugeordneten Oberflächen erzeugt Kontaktkräfte.

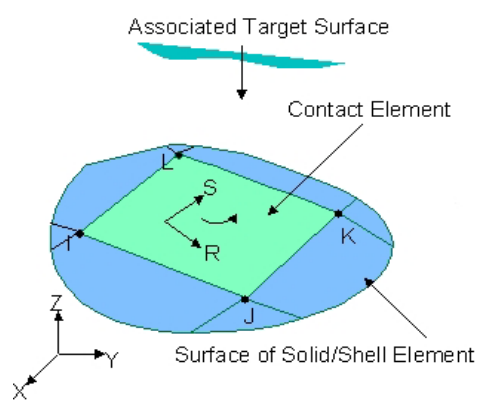

Bild 3.30: Elementtyp CONTA173 [1]

Entsprechend der Belastungsabfolge der experimentellen Untersuchungen wird beim Finite-Element-Modell zuerst die flächige Auflast über die Knoten der oberen Sandwichdeckschicht aufgebracht. Zu dieser Vorbelastung erfolgt im nächsten 
Schritt die Auslenkung des Pfettenprofils durch das in der Drehachse wirkende Drehmoment. Das Drehmoment wird dabei inkrementell über mindestens 10 Laststufen aufgebracht. Sowohl die Größe der Auflast als auch die des Drehmoments sind frei wählbar und können den Vorgaben aus den Versuchen angepasst werden.

Bild 3.31 zeigt die Verformungsfigur des Finite-Element-Modells aus Bild 3.27 in Elementdickenrichtung ( $\mathrm{y}$-Richtung) nach zuerst flächiger Vorbelastung (Auflast $p=3,0 \mathrm{kN} / \mathrm{m}$ ) und anschließender Verdrehung des Pfettenprofils entgegen des Uhrzeigersinns durch $m_{t}=0,3 \mathrm{kNm} / \mathrm{m}$.

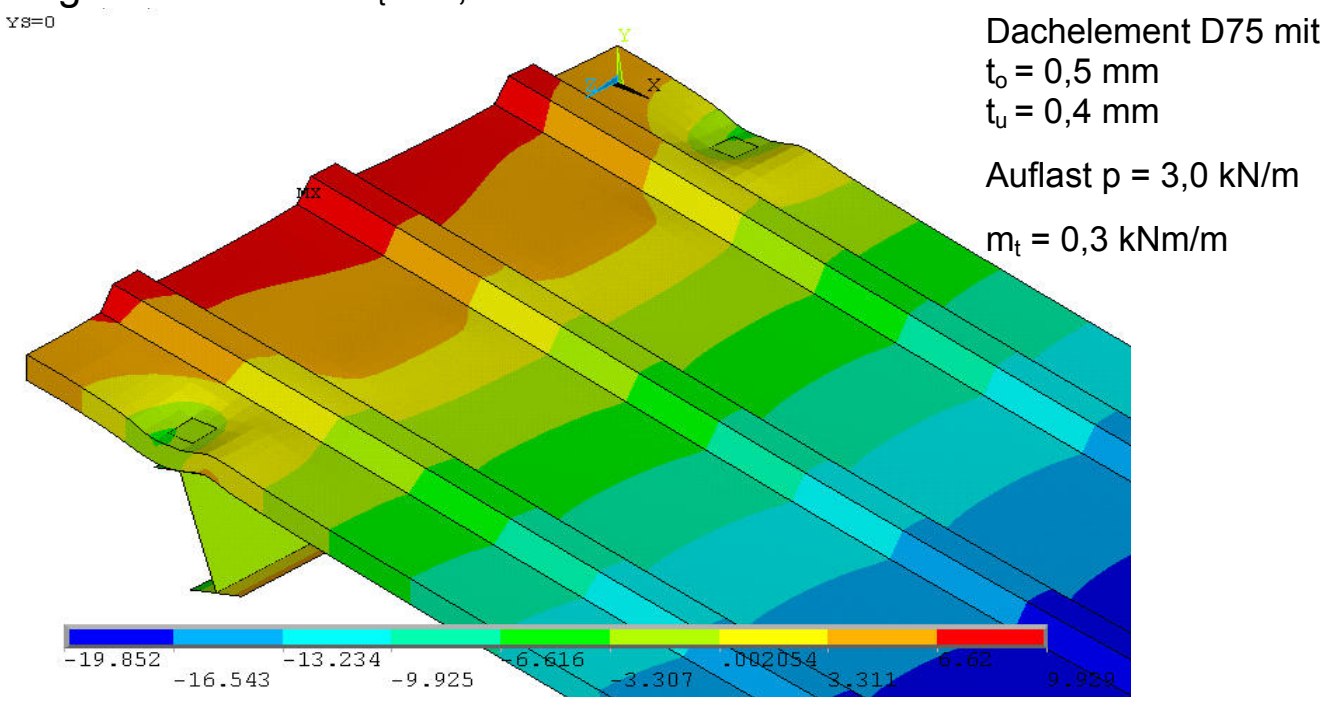

Bild 3.31: Verformungsfigur des Finite-Element-Modells, Verformungen in mm

\subsubsection{Voruntersuchungen}

\subsubsection{Allgemeines}

Damit umfangreiche experimentelle Untersuchungen durch Finite-ElementBerechnungen ersetzt werden können, muss vorab sichergestellt werden, dass das Finite-Element-Modell zuverlässige Ergebnisse liefert. Neben Untersuchungen zur Ermittlung der erforderlichen Netzdichte (Netz-Konvergenz-Studien) sind bei dem im Rahmen dieser Untersuchungen verwendeten Modell insbesondere Studien bezüglich einer korrekten Erfassung des komplexen Materialverhaltens der Sandwichkernschicht notwendig. Ein Vergleich mit analytischen Rechenmethoden in Bezug auf das Biegetragverhalten des Sandwichelementes ist zudem sinnvoll, um eventuell auftretende Schubversteifungseffekte der verwendeten Volumenelemente ausschließen zu können. Ergänzt werden die Voruntersuchungen durch Betrachtung des Verformungszustandes der ausgelenkten Pfette. Damit wird gezeigt, inwieweit eine gegebenenfalls auftretende Profilverformung die Auswertung im Hinblick auf die Anschlusssteifigkeit beeinflusst.

\subsubsection{Biegetragverhalten des Sandwichelementes}

Zur Untersuchung, ob die Modellierung eines Sandwichelementes aus Volumen- und Schalenelementen das Verhalten unter Biegebeanspruchung korrekt wiedergibt, wurde ein Balkenmodell berechnet und mit der bekannten analytischen Lösung zur Ermittlung der Durchbiegung in Feldmitte verglichen. Unerwünschte Schubversteifungseffekte (shear-locking) im Finite-Element-Modell lassen sich durch dieses Vor- 
gehen feststellen. Hierzu wird das in Bild 3.32 dargestellte statische System eines Biegebalkens mit Sandwichquerschnitt unter Gleichstreckenlast betrachtet:

\section{Statisches System}

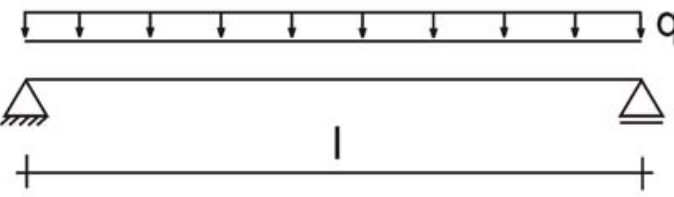

\section{Schnittgrößen}
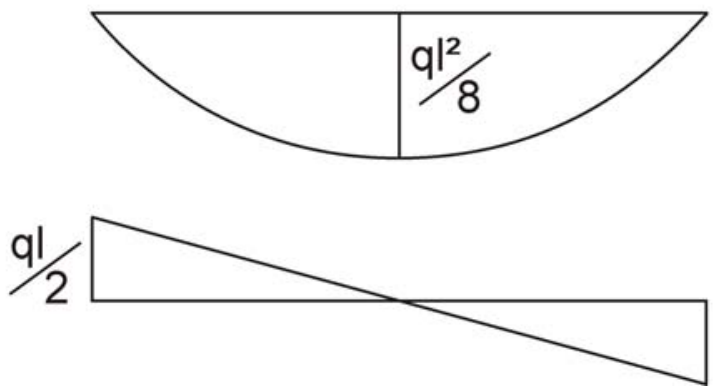

\section{Querschnitt}

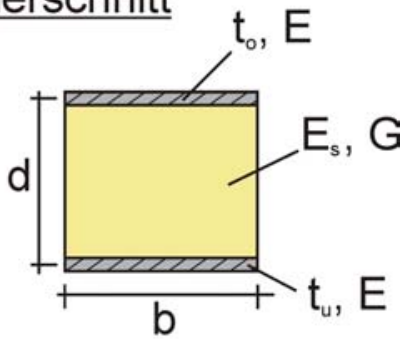

M-Linie

Q-Linie

Bild 3.32: System eines Biegebalkens mit Sandwichquerschnitt

Aufgrund des deutlich kleineren Elastizitätsmoduls der Kernschicht im Vergleich zu dem der Stahldeckschichten sind die Biegenormalspannungen im Schaum vernachlässigbar klein. Entsprechend Bild 3.33 kann der Lastabtrag der Biegemomente in guter Näherung als Biegenormalspannungen über die Deckschichten und der der Querkräfte als Schubspannungen in der Kernschicht erfolgen [51].
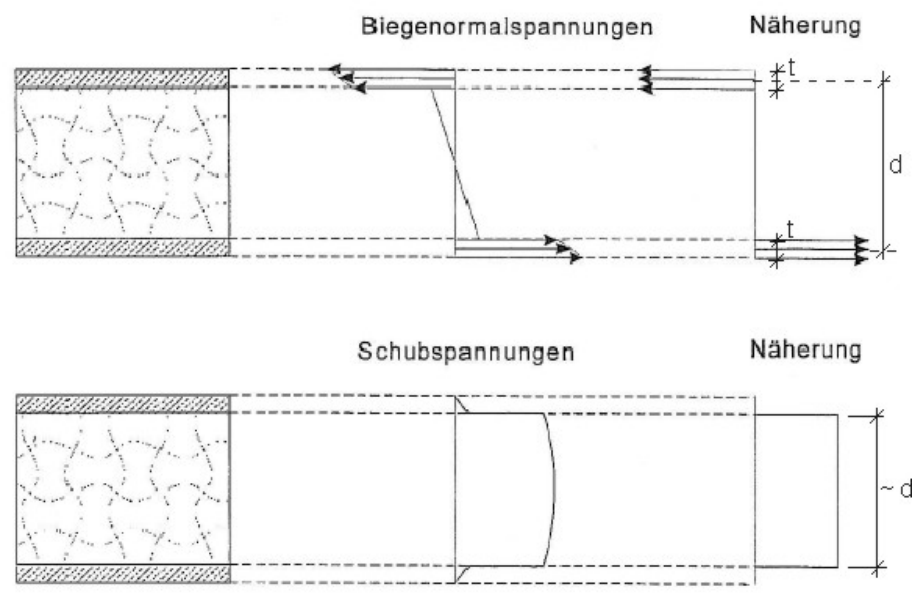

Bild 3.33: angenäherte Spannungsverteilung im biegebeanspruchten Sandwichbalken

Damit ergeben sich die Normalspannungen in den Deckschichten zu

$$
\sigma_{\text {oben }}=\sigma_{\text {unten }}=\frac{M}{d \cdot t \cdot b}=\frac{q \cdot l^{2}}{8 \cdot d \cdot t \cdot b}
$$

Die zugehörige Durchbiegung in Feldmitte setzt sich entsprechend Gleichung (3.6) aus einem Biegeanteil $w_{B}$ und einem Schubanteil $w_{S}$ zusammen. 


$$
w=w_{B}+w_{S}=\frac{5 \cdot q \cdot l^{4}}{384 \cdot E I}+\frac{q \cdot l^{2}}{8 \cdot G A}
$$

mit E Elastizitätsmodul der Deckschichten

I Trägheitsmoment der Deckschichten

G Schubmodul der Kernschicht

A Querschnittsfläche der Kernschicht

Die Belastung des Finite-Element-Modells erfolgte durch eine Flächenlast q, deren Größe so gewählt wurde, dass sich im Balkenmodell entsprechend Gleichung (3.5) eine Biegenormalspannung von $100 \mathrm{~N} / \mathrm{mm}^{2}$ in den Deckschichten ergibt. Das untersuchte Finite-Element-Modell ist in Bild 3.34 mit der resultierenden Verformungsfigur und dem Spannungsplot in Balkenlängsrichtung dargestellt.
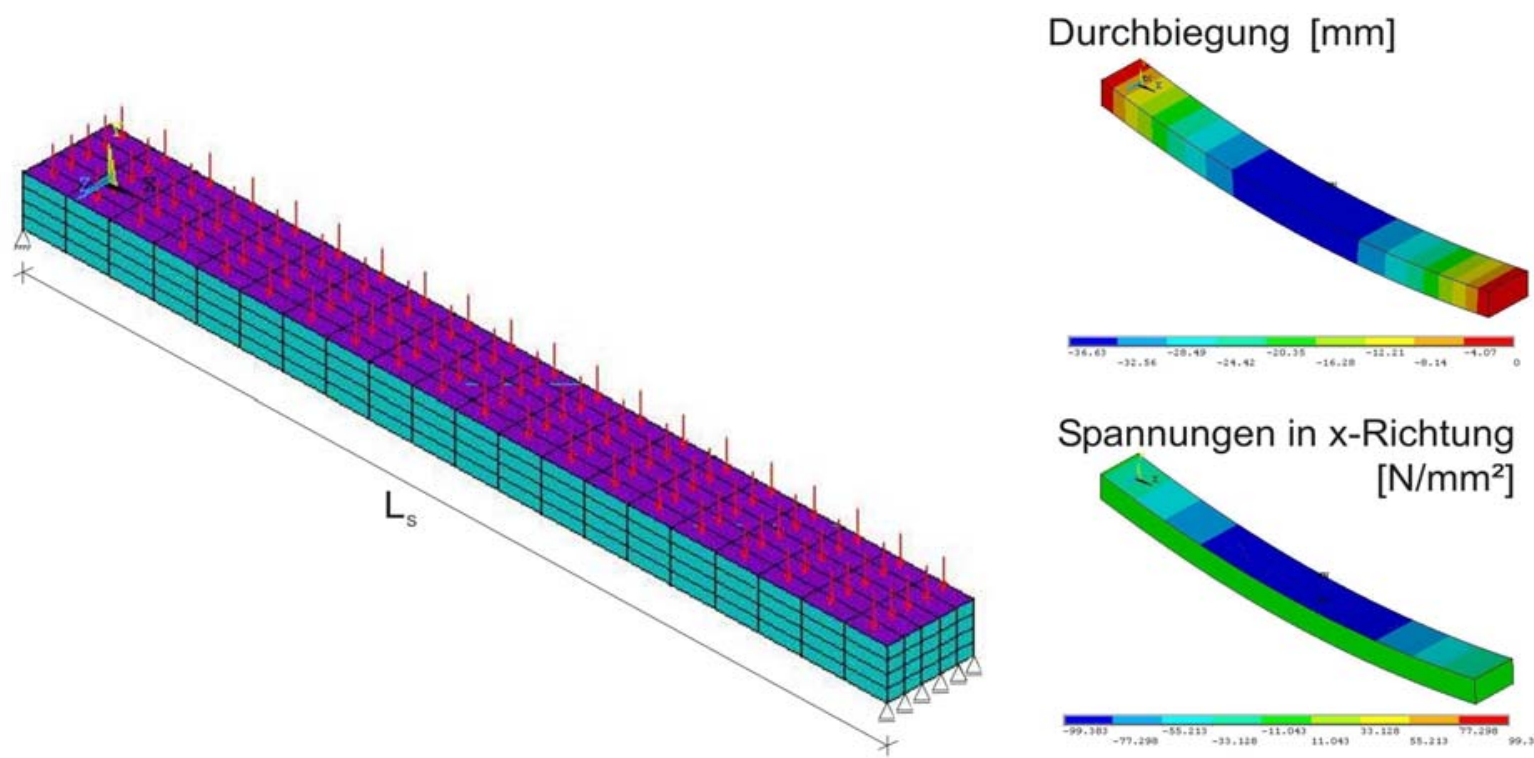

Spannungen in x-Richtung

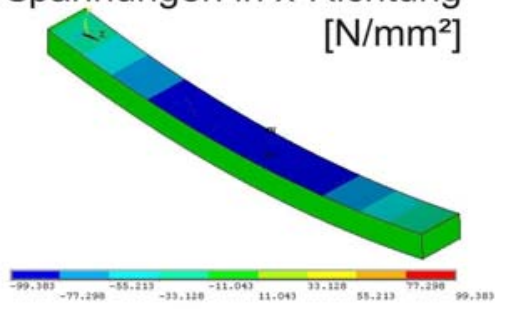

Bild 3.34: FE-Balkenmodell mit Verformungsfigur und Spannungsplot in x-Richtung

Mit den Parametern $E=210000 \mathrm{~N} / \mathrm{mm}^{2}, G=2 \mathrm{~N} / \mathrm{mm}^{2}, t_{o}=t_{u}=0,7 \mathrm{~mm}, b=100 \mathrm{~mm}$ und $L_{s}=1000 \mathrm{~mm}$ ergeben sich die in der Tabelle 3.5 für den Dickenbereich $40 \mathrm{~mm} \leq \mathrm{d} \leq 100 \mathrm{~mm}$ aufgeführten Biegenormalspannungen $\sigma$ und Durchbiegungen w.

\begin{tabular}{|c|c|c|c|c|c|c|c|c|c|}
\hline \multirow{2}{*}{$\begin{array}{c}\text { Elementdicke } \\
\mathrm{d}\end{array}$} & \multirow{2}{*}{$\begin{array}{c}\text { Flächenlast } \\
\text { q }\end{array}$} & \multirow{2}{*}{$\begin{array}{l}\text { Trägheits- } \\
\text { moment I }\end{array}$} & \multicolumn{3}{|c|}{ Balkentheorie } & \multicolumn{2}{|c|}{ FE-Berechnung } & \multicolumn{2}{|c|}{ Abweichungen } \\
\hline & & & $w_{b}$ & $w_{s}$ & w & $\sigma$ & w & $\sigma$ & w \\
\hline$[\mathrm{mm}]$ & {$\left[\mathrm{N} / \mathrm{mm}^{2}\right]$} & {$\left[\mathrm{mm}^{4}\right]$} & {$[\mathrm{mm}]$} & [mm] & {$[\mathrm{mm}]$} & {$\left[\mathrm{N} / \mathrm{mm}^{2}\right]$} & [mm] & [\%] & [\%] \\
\hline 40 & 0,0224 & 56000 & 2,5 & 35 & 37,5 & 99,3 & 37,3 & 0,7 & $-0,5$ \\
\hline 60 & 0,0336 & 126000 & 1,1 & 35 & 36,1 & 99,4 & 36,6 & 0,6 & 1,4 \\
\hline 80 & 0,0448 & 224000 & 0,6 & 35 & 35,6 & 99,4 & 36,4 & 0,6 & 2,2 \\
\hline 100 & 0,0560 & 350000 & 0,4 & 35 & 35,4 & 99,4 & 36,3 & 0,6 & 2,6 \\
\hline
\end{tabular}

Tabelle 3.5: Vergleich von Spannungen und Durchbiegungen nach Theorie und FE-Berechnung

Die Geringfügigkeit der Abweichungen bei den ermittelten Spannungen und Durchbiegungen zeigt, dass das gewählte Finite-Element-Modell das Verhalten eines Sandwichelementes unter Biegebeanspruchung korrekt wiedergibt und etwaige Schubversteifungseffekte ausgeschlossen werden können. 


\subsubsection{Verformungsverhalten des Pfettenprofils}

Entsprechend Gleichung (3.3) kann die Anschlusssteifigkeit durch die Profilverformung des gestützten Trägers beeinflusst werden. Sowohl den Untersuchungen nach [35] als auch der Auswertung der Versuche nach Abschnitt 3.1 liegt die Annahme zugrunde, dass für die Ermittlung der Anschlusssteifigkeit die Profilverformung von warmgewalzten I-Trägern näherungsweise vernachlässigt werden kann.

Im Hinblick auf die Auswertung nachfolgend beschriebener Finite-ElementBerechnungen ist eine geeignete Methode zur Ermittlung der Pfettenverdrehung notwendig. Dazu wird anhand des in Abschnitt 3.2.2 mit Bild 3.27 beschriebenen Modells sowohl die Profilverformung als auch eine gegebenenfalls vorhandene Verwindung des Profils untersucht. Letzteres tritt im Fall einer alternierenden Anordnung der Verbindungsmittel auf.

Dieser Sachverhalt wurde an einem modellierten Pfettenprofil IPE160 (Stahlsorte S235GD) in Kombination mit einem Dachelement mit 40 mm dicker Kernschicht, Elastizitätsmodul von $E_{s}=5,0 \mathrm{~N} / \mathrm{mm}^{2}$ und Deckschichtdicken $t_{o}=t_{u}=0,7 \mathrm{~mm}$ unter Berücksichtigung einer alternierenden Befestigung und einer Pfettenauflast von $6,5 \mathrm{kN} / \mathrm{m}$ untersucht. Es ergaben sich bei Belastung $m_{t}=0,44 \mathrm{kNm} / \mathrm{m}$ die in Tabelle 3.6 aufgeführten Knotenverdrehungen um die Pfettenlängsachse für den Obergurt, den Steg und für das gesamte Pfettenprofil.

\begin{tabular}{|c|c|c|c|}
\hline Verdrehungen [rad] & Steg & Obergurt & gesamtes Profil \\
\hline Minimalwert $\vartheta_{\min }$ & 0,2045 & 0,2027 & 0,2027 \\
\hline Maximalwert $\vartheta_{\max }$ & 0,2260 & 0,2260 & 0,2260 \\
\hline Differenz $\Delta \vartheta=\vartheta_{\max }-\vartheta_{\min }$ & 0,0215 & 0,0233 & 0,0233 \\
\hline Mittelwert $\vartheta_{m}$ & 0,2196 & 0,2192 & 0,2195 \\
\hline
\end{tabular}

Tabelle 3.6: Verdrehungen der Knoten im Steg und Obergurt des Pfettenprofils

Die ermittelten Werte zeigen, dass die größten Unterschiede zwischen Minimal- und Maximalverdrehung im Obergurt auftreten. Bezogen auf den Mittelwert ergibt sich als minimaler Unterschied

$$
\Delta_{\text {min }}=\frac{\vartheta_{\text {min }}-\vartheta_{m}}{\vartheta_{m}}=\frac{0,2027-0,2192}{0,2192} \cdot 100=-7,5 \%
$$

und als maximaler Unterschied

$$
\Delta_{\max }=\frac{\vartheta_{\max }-\vartheta_{m}}{\vartheta_{m}}=\frac{0,2260-0,2192}{0,2192} \cdot 100=3,1 \%
$$

Diese geringen Unterschiede können bei der Ermittlung der Pfettenverdrehung vernachlässigt werden, zumal sie in dieser Größenordnung erst bei relativ großen Verdrehungen auftreten. In dem für die Auswertung relevanten Bereich (Verdrehungen $\vartheta \leq 0,1 \mathrm{rad}$ ) werden sich diese Unterschiede nochmals deutlich verringern.

Ein Vergleich der in Tabelle 3.6 aufgeführten Werte für Steg, Obergurt und das gesamte Profil zeigt nahezu keine Abweichungen beim Mittelwert. Aufgrund dieser Tat- 
sache werden die folgenden Finite-Element-Berechnungen vereinfacht mit dem im Steg ermittelten Mittelwert der Verdrehung ausgewertet. Der mit diesem Vorgehen entstehende Fehler ist vernachlässigbar klein.

Damit ist auch gezeigt, dass die Vernachlässigung der Profilverformung beim Profil IPE 160 erwartungsgemäß gerechtfertigt ist.

\subsubsection{Werkstoffgesetz der Sandwichkernschicht}

Durch den Aufschäumvorgang liegt bei Sandwichelementen mit Kernschicht aus Polyurethan-Hartschaum eine ausgeprägte Richtungsabhängigkeit der mechanischen Eigenschaften vor. Untersuchungen an Schaumwürfeln mit den Abmessungen $100 \mathrm{~mm} \times 100 \mathrm{~mm}$ x Elementdicke zeigten, dass der Schaum in Produktionsrichtung eine erheblich größere Steifigkeit und Festigkeit aufweist, als in den beiden dazu rechtwinkligen Richtungen [20]. Dieses als transversale Isotropie bezeichnete Verhalten wird durch die Längsorientierung der Schaumzellen begründet (siehe Bild 3.35).

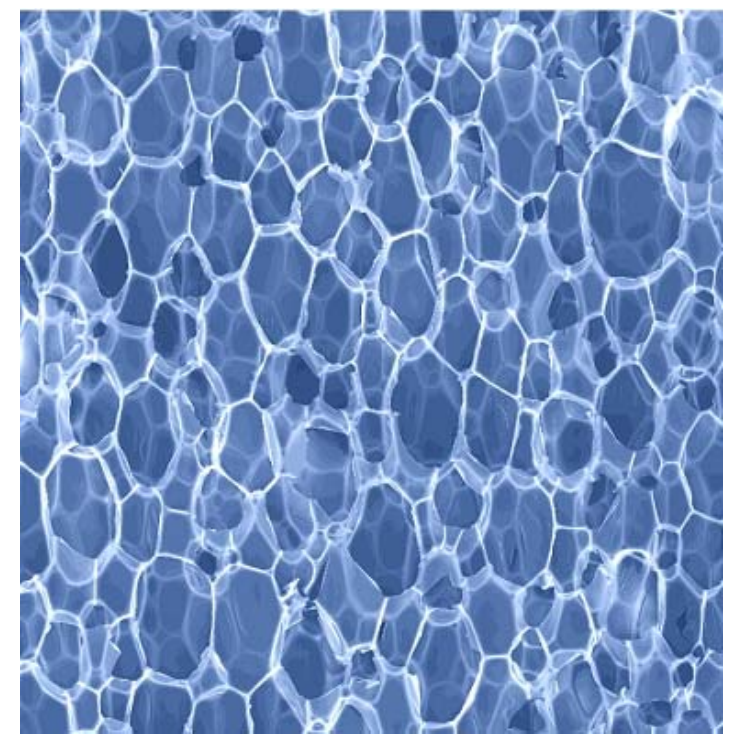

Bild 3.35: Makroaufnahme der Zellstruktur

Analytische Untersuchungen [24] unter Berücksichtigung eines homogenen und orthotropen Kontinuums zeigen, dass die Tragfähigkeit von Sandwichelementen im Hinblick auf das Elementknittern hauptsächlich durch den Elastizitätsmodul $E_{S}$ in Dickenrichtung sowie den Schubmodul $\mathrm{G}_{s}$ rechtwinklig zur Elementebene beeinflusst werden. Der wesentlich größere Elastizitätsmodul in Produktionsrichtung der Elemente kann vernachlässigt werden. Numerische Untersuchungen [56] bestätigten diesen Sachverhalt und verdeutlichten zudem, dass auch variierte Querdehnzahlen einen vernachlässigbaren Einfluss haben.

Zusätzlich zu den zuvor genannten anisotropen Eigenschaften weist die Polyurethan-Kernschicht eine ausgeprägte Inhomogenität in Dickenrichtung auf, da die Schaumdichte im deckschichtnahen Bereich fertigungsbedingt deutlich größer ist als in Kernmitte. Da eine große Schaumdichte die mechanischen Eigenschaften der Kernschicht begünstigt, ist damit zurechnen, dass die inhomogene Verteilung auch auf die Modulwerte zutrifft. 
In den allgemeinen bauaufsichlichen Zulassungen von Sandwichelementen sind zur kontinuierlichen Sicherstellung der mechanischen Eigenschaften im Rahmen der werkseigenen Produktionskontrolle unter anderem Zug- und Druckversuche an Probewürfeln aus der Kernschicht gefordert. Entsprechend den Ausführungen aus Abschnitt 3.1.6.3 werden dabei sowohl der Zugmodul $E_{Z}$ als auch der Druckmodul $E_{D}$ als integrale Werte über die Dicke des Sandwichelementes bestimmt. Der Bemessung von Sandwichelementen genügen diese Werte, eine Aussage über die inhomogene Verteilung der Modulwerte und deren Auswirkung bei lokalen Belastungen wie sie bei der Drehbettungswirkung auftreten, ist damit jedoch nicht möglich.

Zur Bestimmung der Verteilung der Modulwerte wurden an der Versuchsanstalt sowohl Zugversuche nach DIN EN 1607 und Druckversuche nach DIN EN 826 unter Verwendung eines optischen Messverfahrens zur Bestimmung der Formänderungen des Schaumkerns durchgeführt [57a]. Dazu wurden auf jeweils drei Zug- und Druckprobekörpern eines Wandelements mit $100 \mathrm{~mm}$ nomineller Dicke Messmarken im Abstand von etwa $10 \mathrm{~mm}$ in Dickenrichtung angebracht. Mit der kalibrierten optischen Messvorrichtung, Typ CCD 1300B der Firma Messtek, wurden auf Basis einer videoprogrammierten Dehnungsmessung die Koordinatenänderung der Messmarken in der Bildebene kontinuierlich in Abhängigkeit der Belastung gemessen. Aus diesen Messwerten wurden die Modulwerte abschnittsweise über die Elementdicke jeweils aus dem linearen Anstieg der Last-Verformungslinie ermittelt.

Bild 3.36 zeigt die Verteilung des Druck-Elastizitätsmoduls $E_{D}$ über die Schaumkerndicke für die drei untersuchten Druckproben sowie den zugehörigen Mittelwert. Die zuvor vermutete ungleichförmige Verteilung der Modulwerte wird bestätigt. In den deckschichtnahen Bereichen treten größere Modulwerte auf, wobei insbesondere die Modulwerte auf der beim Produktionsprozess untenliegenden Deckschichtseite deutlich größer sind als die auf der gegenüberliegenden Seite. An Elementen unterschiedlicher Dicke durchgeführte alternative Druckversuche [56] bestätigen große Modulwerte speziell im Bereich der unteren Deckschicht bis zu einer bezogenen Schaumkerndicke $\mathrm{h} / \mathrm{d}=0.25$.

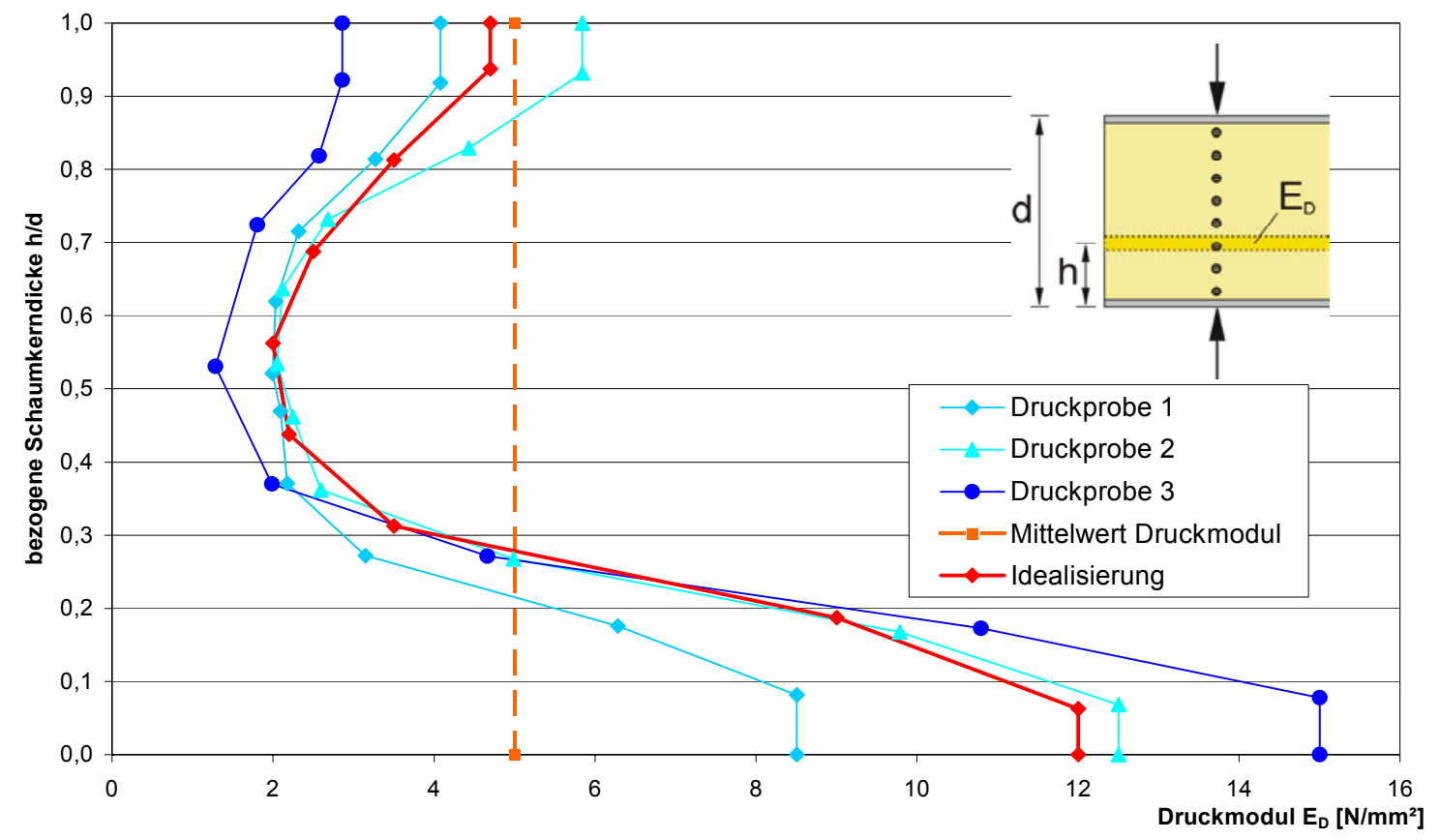

Bild 3.36: Verteilung des Druck-Elastizitätsmoduls $E_{D}$ über die Schaumkerndicke 
Bild 3.37 zeigt die Verteilung des Zug-Elastizitätsmoduls $E_{Z}$ über die Schaumkerndicke für die drei untersuchten Zugproben sowie den zugehörigen Mittelwert. Auch hier zeigt sich eine ungleichförmige Verteilung der Modulwerte, wobei die größeren Modulwerte im Gegensatz zu der Druckbeanspruchung lediglich im Bereich der beim Produktionsprozess untenliegenden Deckschichtseite auftreten.

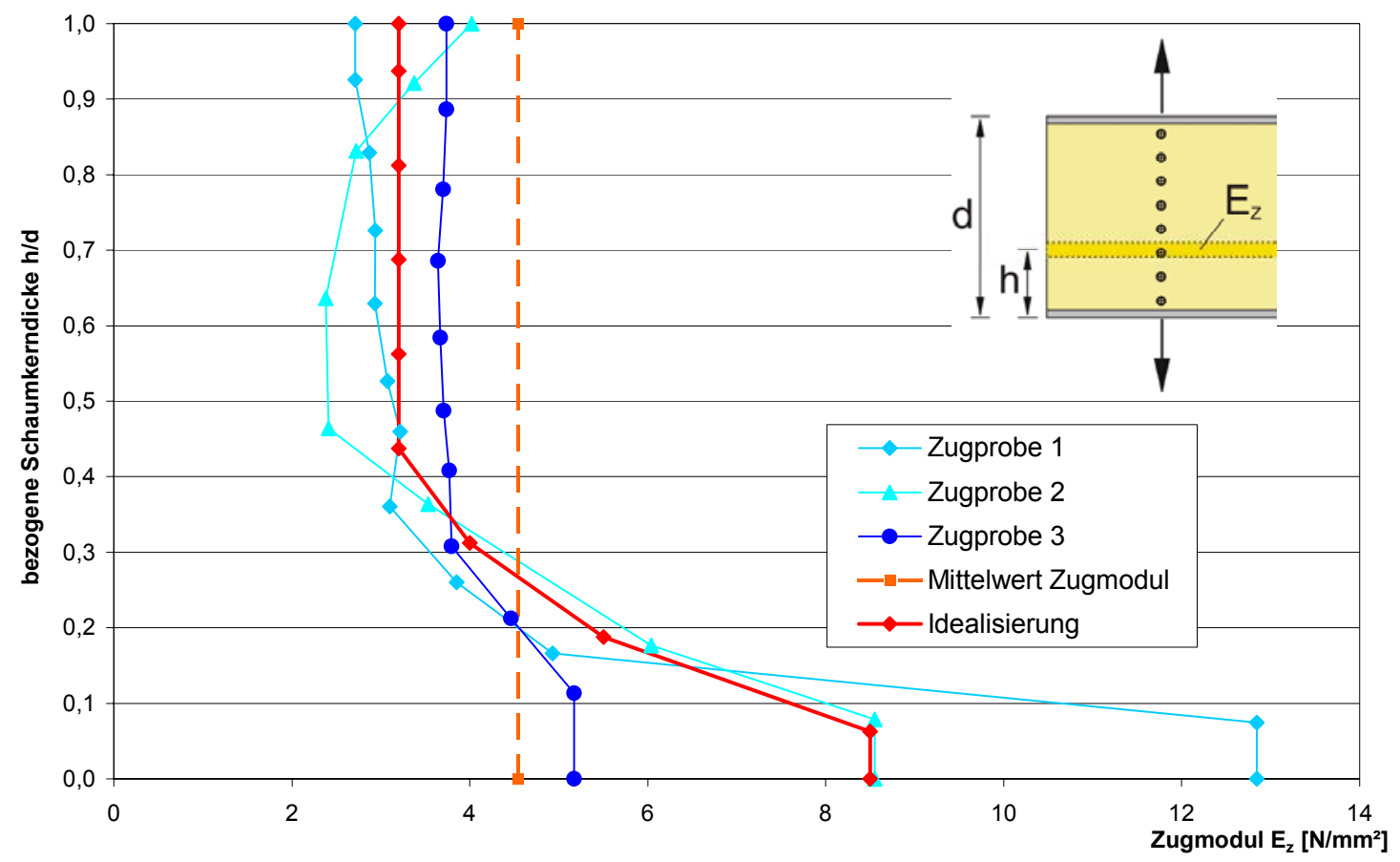

Bild 3.37: Verteilung des Zug-Elastizitätsmoduls $E_{z}$ über die Schaumkerndicke

Zur numerischen Untersuchung des Einflusses der Verteilung der Modulwerte werden die jeweils drei Kurvenverläufe in Bild 3.36 und Bild 3.37 idealisiert. Der gemittelte Verlauf von Zug- und Druckmodulverteilung über die bezogene Schaumkerndicke $\mathrm{h} / \mathrm{d}$ wird als idealisierter Elastizitätsmodul bezeichnet und ist zusammen mit dem homogenisierten Elastizitätsmodul in Bild 3.38 dargestellt.

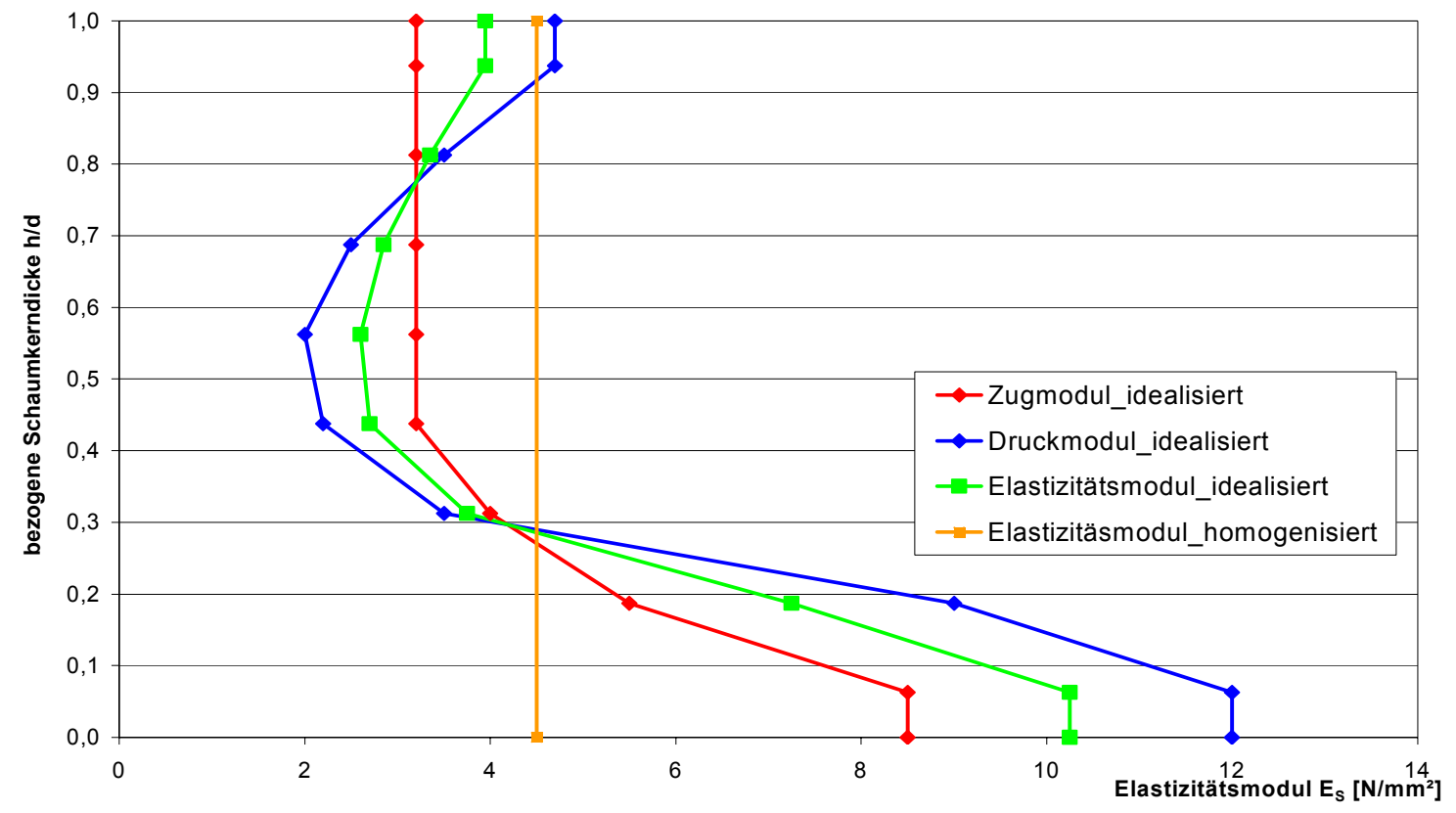

Bild 3.38: idealisierter und homogenisierter Elastizitätsmodul $E_{S}$ über die Schaumkerndicke 
Mit dem im Abschnitt 3.2.2 mit Bild 3.27 beschriebenen Finite-Element-Modell wurden Berechnungen an einem Wandelement mit $80 \mathrm{~mm}$ dicker Kernschicht, Deckschichtdicken $t_{o}=0,54 \mathrm{~mm}$ und $t_{u}=0,46 \mathrm{~mm}$ und einer Pfettenauflast von $3,5 \mathrm{kN} / \mathrm{m}$ unter Verwendung des idealisierten sowie des homogenisierten Elastizitätsmoduls durchgeführt. Dazu wurde den abschnittsweise definierten Elementeigenschaften der Schaumschicht die in Bild 3.38 dargestellten Verläufe des Elastizitätsmoduls zugewiesen. Die daraus resultierenden, in Bild 3.39 dargestellten MomentenVerdrehungsbeziehungen werden entsprechend dem Verfahren nach Abschnitt 3.1.7 ausgewertet. Es ergeben sich die in Tabelle 3.7 aufgeführten Werte für $\mathbf{c}_{91}$ und $c_{92}$.

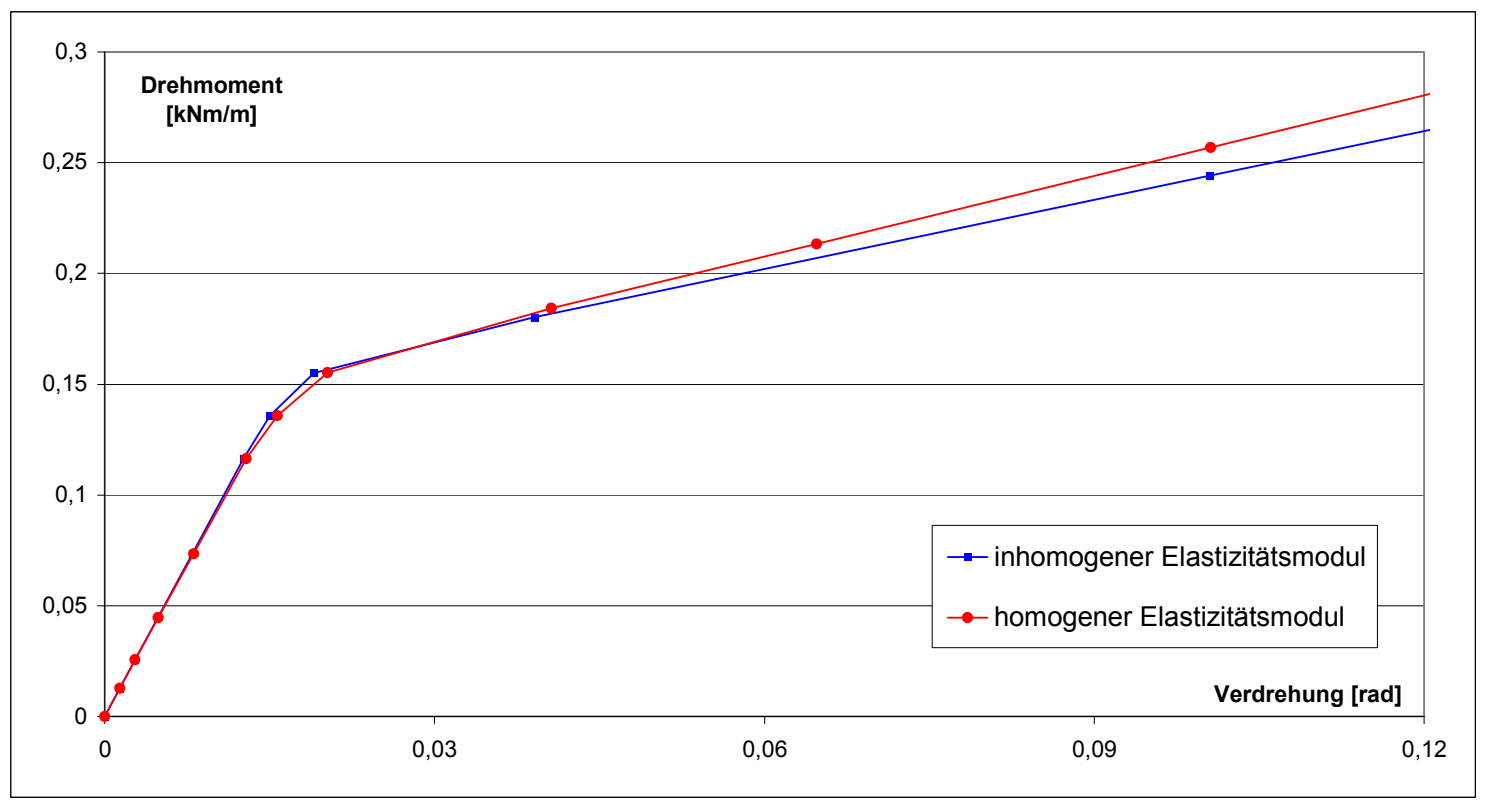

Bild 3.39: Momenten-Verdrehungsbeziehungen für homogenen und inhomogenen Verlauf von $E_{S}$

\begin{tabular}{|c|c|c|}
\hline & $\mathrm{c}_{91}[\mathrm{kNm} / \mathrm{m}]$ & $\mathrm{c}_{92}[\mathrm{kNm} / \mathrm{m}]$ \\
\hline homogener Verlauf von $\mathrm{E}_{\mathrm{S}}$ & 9,16 & 1,21 \\
\hline inhomogener Verlauf von $\mathrm{E}_{\mathrm{S}}$ & 9,25 & 1,09 \\
\hline \hline Unterschied [\%] & -1 & 11 \\
\hline
\end{tabular}

Tabelle 3.7: Werte $c_{91}$ und $c_{\vartheta 2}$ für homogenen und inhomogenen Verlauf von $E_{S}$

Der Vergleich der in Tabelle 3.7 aufgeführten Werte zeigt, dass der Wert $\mathbf{c}_{91}$ nahezu nicht und der Wert $\mathbf{c}_{\vartheta 2}$ nur geringfügig durch die hier untersuchte inhomogene Verteilung der Elastizitätsmoduli beeinflusst wird. Die Abweichungen der Werte $\mathbf{C}_{\vartheta 1}$ und $\mathbf{C}_{\vartheta 2}$ wirken sich in dem hier dargestellten Fall lediglich ab Verdrehungen $\vartheta>0,05$ rad erkennbar auf den Verlauf der Momenten-Verdrehungsbeziehung aus. Für die Ermittlung der als Sekante gegebene Anschlusssteifigkeit spielt der Kurvenverlauf nach Überschreitung des Kontaktmomentes $m_{k}$ eine untergeordnete Bedeutung. Aus diesen Gründen und weil die Bestimmung der Modulwertverteilung über die Elementdicke ein sehr aufwändiges, in der werkseigenen Produktionskontrolle der Hersteller unpraktikables Verfahren ist, kann die inhomogene Modulwertverteilung vereinfacht durch eine homogene Verteilung angenähert werden. Hierzu kann beispielsweise der im Rahmen der werkseigenen Produktionskontrolle der Hersteller ermittelte integrale Wert des Elastizitätsmoduls $E_{S}$ angesetzt werden. 
Bei allen weiteren Finite-Element-Berechnungen wird aufgrund der zuvor dargestellten Ergebnisse die Kernschicht mit einem homogenen und isotropen Werkstoffgesetz abgebildet.

\subsubsection{Verifizierung der Finite-Element-Modellierung}

In diesem Abschnitt wird die Güte des in Abschnitt 3.2.2 beschriebenen FiniteElement-Modells beurteilt. Zu diesem Zweck werden zwei ausgewählte Versuche aus Abschnitt 3.1 mit dem Finite-Element-Modell nachgerechnet und verglichen. Es wird jeweils ein Dach- und ein Wandelement unter Berücksichtigung der in Abschnitt 3.1.6 aufgeführten Materialkennwerte modelliert. Die Kernschicht wird dabei mit einem homogenen und isotropen Werkstoffgesetz abgebildet. Die experimentell bei unterschiedlichen Auflasten mit jeweils vier Zyklen ermittelten MomentenVerdrehungsdiagramme werden damit verglichen.

Für das in dem Versuch D5/PUR untersuchte Dachelement erfolgt der Vergleich mit den Ergebnissen der Finite-Element-Berechnung in Bild 3.40 und für das in dem Versuch W1/PUR untersuchte Wandelement in Bild 3.41. 


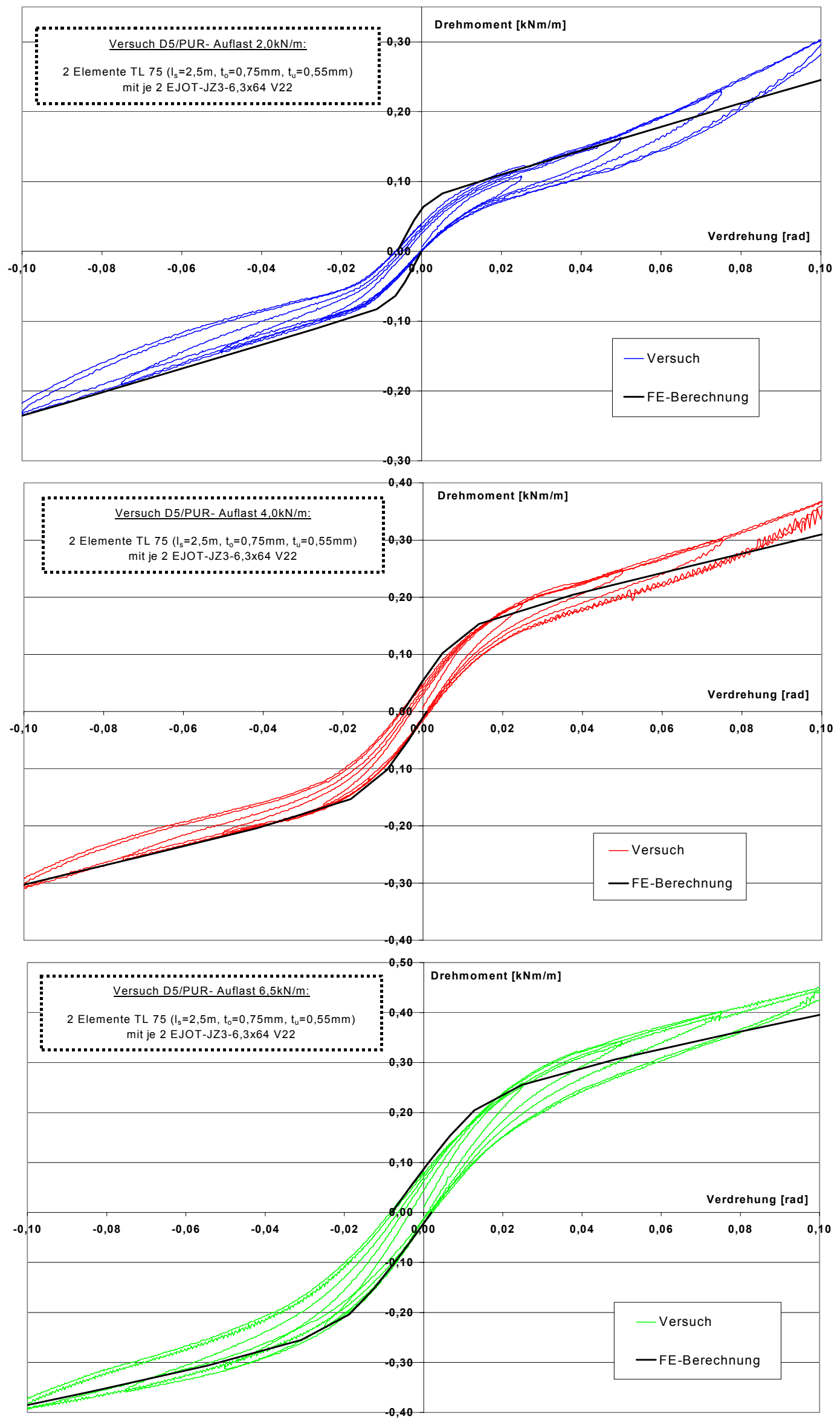

Bild 3.40: Gegenüberstellung der Momenten-Verdrehungsbeziehung nach Versuch D5/PUR und Finite-Element-Berechnung 

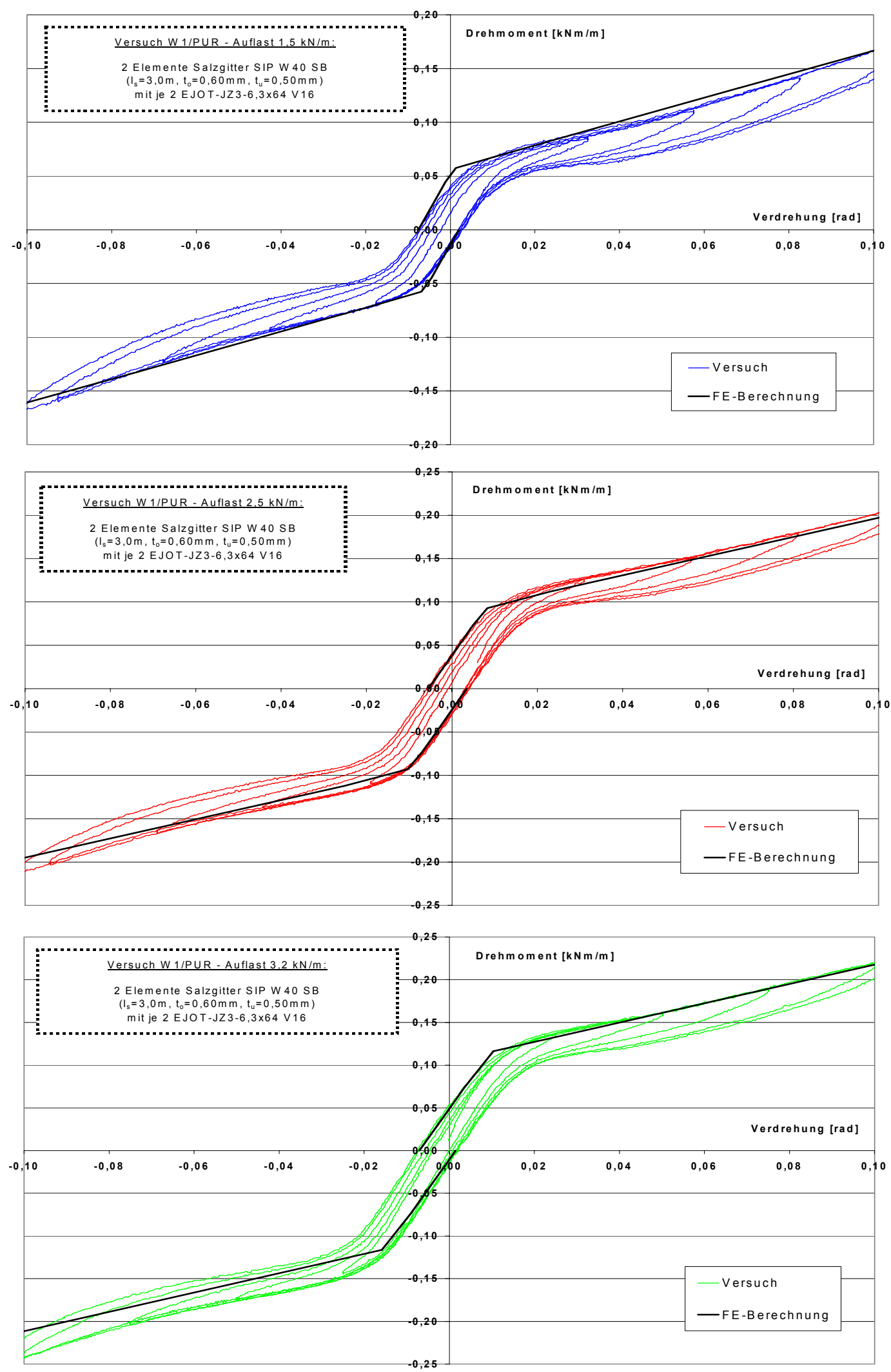

Bild 3.41: Gegenüberstellung der Momenten-Verdrehungsbeziehung nach Versuch W1/PUR und Finite-Element-Berechnung

Die in Bild 3.40 und Bild 3.41 dargestellten numerisch ermittelten MomentenVerdrehungsbeziehungen stimmen qualitativ gut mit den Beziehungen aus den Versuchen überein. Die Beurteilung der Güte des Finite-Element-Berechnung erfolgt 
durch Vergleich der numerisch und experimentell ermittelten MomentenVerdrehungsbeziehungen bezüglich der Parameter $\mathbf{c}_{91}$ und $\mathbf{c}_{\vartheta 2}$ sowie des Ordinatenwertes des Schnittpunktes der beiden entsprechend Bild 3.26 linearisierten Steifigkeitsbereiche. Die aus Bild 3.40 und Bild 3.41 ermittelten Werte $c_{91}$ und $c_{92}$ sind in Tabelle 3.8, die Kontaktmomente $m_{k}(\exp )$ und $m_{k}(F E M)$ in Tabelle 3.9 zusammengestellt.

\begin{tabular}{|c|c|c|c|c|c|c|}
\hline & \multirow[b]{2}{*}{$\begin{array}{l}\text { Laststufe } \\
\text { Nr. }\end{array}$} & \multirow[b]{2}{*}{$\begin{array}{l}\text { Auflast } \\
{[\mathrm{kN} / \mathrm{m}]}\end{array}$} & \multicolumn{2}{|c|}{$\mathrm{c}_{91}[\mathrm{kNm} / \mathrm{m}]$} & \multicolumn{2}{|c|}{$\mathrm{c}_{92}[\mathrm{kNm} / \mathrm{m}]$} \\
\hline & & & $\begin{array}{l}\text { experimentelle } \\
\text { Untersuchung }\end{array}$ & $\begin{array}{c}\text { FE- } \\
\text { Berechnung }\end{array}$ & $\begin{array}{l}\text { experimentelle } \\
\text { Untersuchung }\end{array}$ & $\begin{array}{c}\text { FE- } \\
\text { Berechnung }\end{array}$ \\
\hline \multirow{2}{*}{ D5/PUR } & 1_pos & 2,0 & 5,90 & & 2,05 & \\
\hline & 1_neg & 2,0 & 6,30 & & 1,75 & \\
\hline \multirow{4}{*}{$\begin{array}{c}\text { Dach- } \\
\text { element } \\
\text { TL75 } \\
\text { nach [5] }\end{array}$} & 2_pos & 4,0 & 8,50 & & 2,17 & \\
\hline & 2_neg & 4,0 & 9,60 & & 1,79 & \\
\hline & 3_pos & 6,5 & 9,50 & & 2,20 & \\
\hline & 3_neg & 6,5 & 10,90 & & 1,59 & \\
\hline \multicolumn{2}{|r|}{ Mittelwerte } & & 8,50 & 9,8 & 1,93 & 1,70 \\
\hline \multicolumn{2}{|c|}{ Abweichung [\%] } & & \multicolumn{2}{|c|}{16} & \multicolumn{2}{|c|}{-12} \\
\hline \multirow{2}{*}{ W1/PUR } & 1_pos & 1,5 & 5,88 & & 0,84 & \\
\hline & 1_neg & 1,5 & 6,12 & & 1,06 & \\
\hline \multirow{4}{*}{$\begin{array}{l}\text { Wand- } \\
\text { element } \\
\text { SIP W40 } \\
\text { nach [6] }\end{array}$} & 2_pos & 2,5 & 6,93 & & 0,99 & \\
\hline & 2_neg & 2,5 & 7,37 & & 1,02 & \\
\hline & 3_pos & 3,2 & 7,20 & & 0,96 & \\
\hline & 3_neg & 3,2 & 7,69 & & 0,95 & \\
\hline \multicolumn{2}{|r|}{ Mittelwerte } & & 6,87 & 6,70 & 0,97 & 1,11 \\
\hline \multicolumn{2}{|c|}{ Abweichung [\%] } & & \multicolumn{2}{|c|}{-2} & \multicolumn{2}{|c|}{14} \\
\hline
\end{tabular}

Tabelle 3.8: Vergleich der experimentell ermittelten Werte $c_{91}$ und $c_{92}$ mit Werten aus der FiniteElement-Berechnung

\begin{tabular}{|c|c|c|c|c|c|c|c|}
\hline & \multirow{2}{*}{$\begin{array}{c}\text { Laststufe } \\
\text { Nr. }\end{array}$} & \multirow{2}{*}{$\begin{array}{c}m_{k} \text { nach } \\
(3.4) \\
{[\mathrm{kNm} / \mathrm{m}]}\end{array}$} & \multicolumn{2}{|c|}{$\begin{array}{l}m_{K}(\exp ) \\
{[\mathrm{kNm} / \mathrm{m}]}\end{array}$} & \multirow{2}{*}{$\begin{array}{c}\mathrm{m}_{\mathrm{K}}(\mathrm{FEM}) \\
{[\mathrm{kNm} / \mathrm{m}]}\end{array}$} & \multicolumn{2}{|c|}{ Abweichungen [\%] } \\
\hline & & & Einzelwerte & Mittelwert & & exp. & FEM \\
\hline \multirow[t]{2}{*}{ D5/PUR } & 1_pos & \multirow{2}{*}{0,082} & 0,090 & \multirow{2}{*}{0,085} & \multirow{2}{*}{0,078} & \multirow{2}{*}{4} & \multirow{2}{*}{-5} \\
\hline & 1_neg & & 0,080 & & & & \\
\hline \multirow{4}{*}{$\begin{array}{c}\text { Dach- } \\
\text { element } \\
\text { TL75 } \\
\text { nach [5] }\end{array}$} & 2_pos & \multirow{2}{*}{0,164} & 0,176 & \multirow{2}{*}{0,165} & \multirow{2}{*}{0,157} & \multirow{2}{*}{1} & \multirow{2}{*}{-4} \\
\hline & 2_neg & & 0,154 & & & & \\
\hline & 3_pos & \multirow{2}{*}{0,267} & 0,291 & \multirow{2}{*}{0,285} & \multirow{2}{*}{0,250} & \multirow{2}{*}{7} & \multirow{2}{*}{-6} \\
\hline & 3_neg & & 0,279 & & & & \\
\hline \multirow{2}{*}{ W1/PUR } & 1 pos & \multirow[b]{2}{*}{0,062} & 0.065 & \multirow[b]{2}{*}{0,063} & \multirow[b]{2}{*}{0,058} & \multirow[b]{2}{*}{2} & \multirow[b]{2}{*}{-7} \\
\hline & 1_neg & & 0,061 & & & & \\
\hline \multirow{4}{*}{$\begin{array}{l}\text { Wand- } \\
\text { element } \\
\text { SIP W40 } \\
\text { nach [6] }\end{array}$} & 2_pos & \multirow{2}{*}{0,103} & 0,108 & \multirow{2}{*}{0,107} & \multirow{2}{*}{0,093} & \multirow{2}{*}{4} & \multirow{2}{*}{-10} \\
\hline & 2_neg & & 0,105 & & & & \\
\hline & 3_pos & \multirow{2}{*}{0,131} & 0,145 & \multirow{2}{*}{0,136} & \multirow{2}{*}{0,122} & \multirow{2}{*}{4} & -7 \\
\hline & 3_neg & & 0,127 & & & & -1 \\
\hline
\end{tabular}

Tabelle 3.9: Vergleich der Werte $m_{K}$ aus experimenteller und Finite-Element-Untersuchung mit den theoretischen Werten nach GI. (3.4)

Der Vergleich der in Tabelle 3.8 aufgeführten Mittelwerte aus experimenteller Untersuchung und Finite-Element-Berechnung zeigt eine maximale Abweichung von $16 \%$ bei $\mathrm{c}_{91}$ und eine maximale Abweichung von $14 \%$ bei $\mathrm{c}_{92}$. Der Vergleich der Einzel- 
werte zeigt beim Versuch D5/PUR (Laststufe 1_pos) mit $66 \%$ Abweichung bei c $_{91}$ sein Maximum. Diese relativ große Abweichung stellt eine Ausnahme dar, da die übrigen festgestellten Abweichungen der Einzelwerte - insbesondere unter größeren Auflasten - maximal $15 \%$ betragen. Zurückzuführen ist diese Ausnahme auf die Folge von Imperfektionen im Schaumkern, die insbesondere bei geringen Auflasten dazu führen können, dass ein geringerer Widerstand des Schaumkerns gegen Eindrücken der Pfette vorhanden ist. Bei größeren Auflasten, die sich im Bereich der zulässigen Druckspannungen des Schaumkerns bewegen, treten diese Abweichungen nicht auf. Dies wird durch den Versuch D2/PUR, der mit größeren Auflasten am gleichen Elementtyp mit identischer Verschraubung durchgeführt wurde, bestätigt.

Beim Wert $\mathbf{c}_{92}$ wurde hinsichtlich der Einzelwerte eine maximale Abweichung von $32 \%$ festgestellt (Versuch W1/PUR, Laststufe 1_pos). Diese maximale Abweichung tritt infolge einer relativ großen Streuung der ermittelten Einzelwerte auf (vgl. Tabelle A5.2 des Anhangs A5 zur statistischen Auswertung der Versuchsergebnisse). Werden die Abweichungen auf die experimentell festgestellten Mittelwerte bezogen, so ergibt sich eine deutlich kleinere Abweichung von etwa $14 \%$.

In Tabelle 3.9 werden die aus Bild 3.40 und Bild 3.41 ermittelten experimentellen und numerischen Kontaktmomente den theoretischen Werten $\mathrm{m}_{\mathrm{K}}$ nach Gleichung (3.4) gegenübergestellt. Bei den Werten $m_{K}(\exp )$ erfolgt der Vergleich mit dem für jede Stufe der Auflast ermittelten Mittelwert. Damit kann der Einfluss einer eventuell vorhandenen Nullpunktverschiebungen aus den Versuchsdiagrammen ausgeschlossen werden. Der Vergleich zeigt minimale Abweichungen bei den Werten $m_{K}(\exp )$ aus den Versuchskurven, die im Bereich der Ablesegenauigkeit liegen. Auch die Abweichungen der numerisch ermittelten Werte $m_{K}(F E M)$ liegen in einem sehr engen Streubereich. Die festgestellten Ergebnisse bestätigen die Gültigkeit des in Abschnitt 3.1.7 dargestellten mechanischen Modells.

\subsubsection{Berechnungen mit dem verifizierten Finite-Element-Modell zur Bestim- mung der signifikanten Einflüsse}

\subsubsection{Allgemeines}

Die Geringfügigkeit der Unterschiede, die sich im Vergleich der Ergebnisse von experimenteller Untersuchung und Finite-Element-Modellierung in Abschnitt 3.2.4 ergibt, zeigt, dass das Finite-Element-Modell für weiterführende Untersuchungen hinsichtlich der drehstabilisierenden Wirkung gut geeignet ist.

In den folgenden Abschnitten wird der Einfluss der Systemparameter auf die gemäß Bild 3.26 definierten Werte $c_{91}, c_{92}$ und $m_{K}$ untersucht, um die signifikanten Einflüsse festzustellen, damit für die statistische Auswertung der in Abschnitt 3.1.7 ermittelten Kennwerte geeignete Populationen gebildet werden können.

\subsubsection{Einfluss des Elementtyps}

Als Sandwich-Dachelemente werden im folgenden solche bezeichnet, bei denen die schraubenkopfseitige Deckschicht trapezprofiliert ausgeführt wird, während als Sandwich-Wandelement solche bezeichnet werden, die beidseitig ebene oder quasiebene Deckschichten haben. 
In diesem Abschnitt wird untersucht, wie sich die drehstabilisierende Wirkung von Dach- und Wandelementen bei gleichen Randbedingungen unterscheidet. Dafür wurde in Kombination mit einem Pfettenprofil IPE160 je ein Dach- und Wandelement mit $40 \mathrm{~mm}$ dicker durchgehender Kernschicht modelliert und mit einer Auflast von $3,0 \mathrm{kN} / \mathrm{m}$ vorbelastet. Der einzige Unterschied zwischen den Berechnungen lag in der Ausbildung der oberen Deckschichtgeometrie. Diese ist für die genannten Elemente in Bild 3.42 dargestellt.

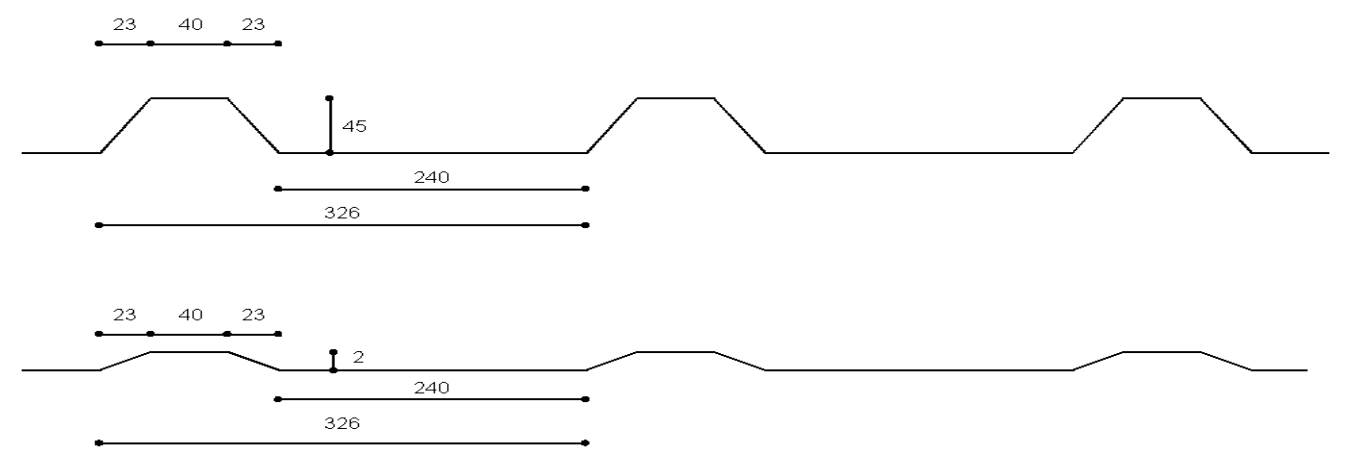

Dachelement mit $\mathrm{h}_{\mathrm{t}}=45 \mathrm{~mm}$

Wandelement mit $h_{t}=2 \mathrm{~mm}$

Bild 3.42: untersuchte obere Deckschichtgeometrie von Dach- und Wandelement

Die numerische Berechnung beider Elementtypen liefert die in Bild 3.43 dargestellten Momenten-Verdrehungsbeziehungen.

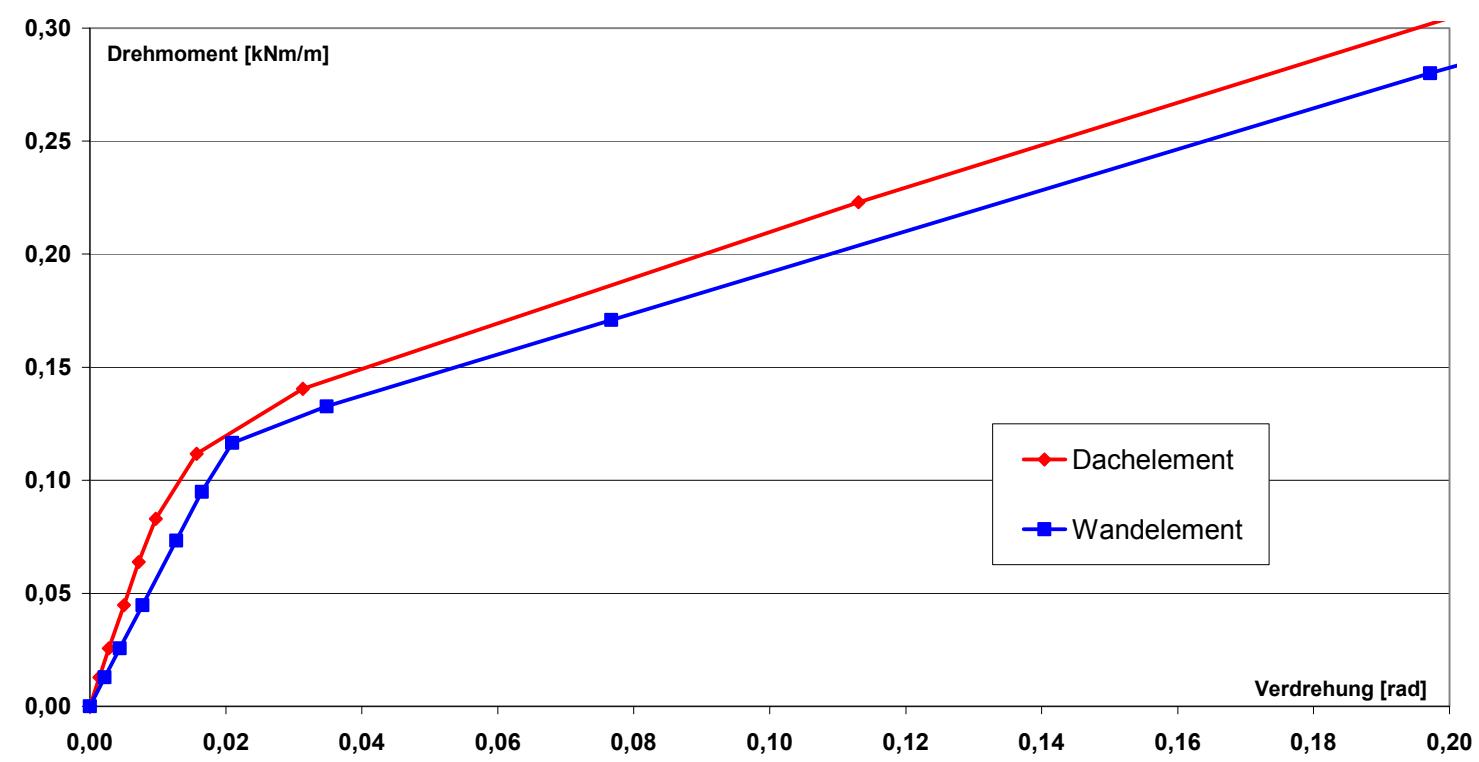

Bild 3.43: Momenten-Verdrehungsbeziehungen für $h_{t}=45 \mathrm{~mm}$ (Dachelement) und $h_{t}=2 \mathrm{~mm}$ (Wandelement)

Bild 3.43 zeigt eine deutlich größere Anfangssteifigkeit für das Dachelement. Der Unterschied in der Kurvensteigung nach Überschreiten des Knickpunktes ist nur geringfügig. Die aus dem Diagramm ermittelten und in Tabelle 3.10 dargestellten Werte $\mathrm{C}_{\vartheta 1}, \mathrm{C}_{\vartheta 2}$ und $\mathrm{c}_{\vartheta \mathrm{A}}$ bestätigen diesen Sachverhalt. 


\begin{tabular}{|c|c|c|c|}
\hline & $\mathrm{c}_{91}[\mathrm{kNm} / \mathrm{m}]$ & $\mathrm{c}_{92}[\mathrm{kNm} / \mathrm{m}]$ & $\mathrm{c}_{\vartheta A}[\mathrm{kNm} / \mathrm{m}]$ \\
\hline Wandelement $\left(\mathrm{h}_{\mathrm{t}}=2 \mathrm{~mm}\right)$ & 5,60 & 0,91 & 6,20 \\
\hline Dachelement $\left(\mathrm{h}_{\mathrm{t}}=45 \mathrm{~mm}\right)$ & 9,10 & 1,01 & 9,70 \\
\hline \hline Unterschied [\%] & 38 & 10 & 36 \\
\hline
\end{tabular}

Tabelle 3.10: Werte $c_{\vartheta 1}$ und $c_{\vartheta 2}$ für Dach- und Wandelement

Der Vergleich der ermittelten Anschlusssteifigkeiten $\mathbf{C}_{\vartheta A}$ mit den Werten $\mathbf{c}_{91}$ zeigt, dass die Anschlusssteifigkeit $\mathrm{C}_{\vartheta \mathrm{A}}$ für den Fall des Wandelements um etwa $10 \%$ und für den Fall des Dachelements um etwa $6 \%$ unterschätzt wird, wenn die Elementbiegesteifigkeit bei der dazu notwendigen Auswertung vernachlässigt wird.

Der Unterschied hinsichtlich des Wertes $\mathbf{c}_{91}$ resultiert aus der erhöhten Steifigkeit durch die rechtwinklig zur Stablängsachse verlaufende Trapezprofilierung der oberen Deckschicht. Das untersuchte Dachelement besitzt eine trapezprofilierte Deckschicht mit drei Hochsicken der Höhe $h_{t}=45 \mathrm{~mm}$. Im Vergleich zu Wandelementen, bei denen eine konstante Bettung über die Querschnittsbreite vorliegt, weisen bei den Dachelementen die Bereiche der Hochsicken eine größere Federsteifigkeit auf. Dies wird durch die Darstellung des mechanischen Modells in Bild 3.44 verdeutlicht. Daraus resultieren die ebenfalls in Bild 3.44 gezeigten Spannungskonzentrationen in der Kernschicht im Bereich der Hochsicken.

\section{Dachelement mit $h_{t}=45 \mathrm{~mm}$}

Querschnitt

mechanisches

Modell
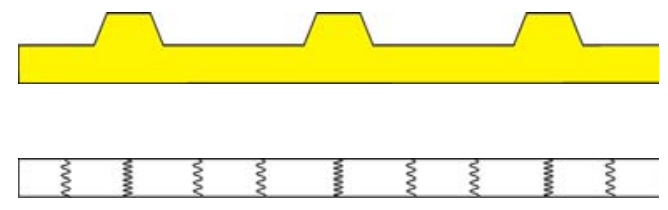

Spannungsverteilung in Dickenrichtung $\left[\mathrm{N} / \mathrm{mm}^{2}\right]$ für $\mathrm{m}_{\mathrm{t}}=0,03 \mathrm{kNm} / \mathrm{m}$

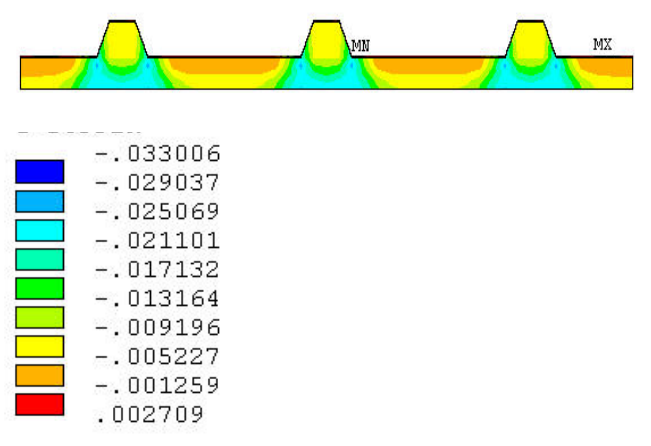

$\underline{\text { Wandelement mit } h_{t}=2 \mathrm{~mm}}$
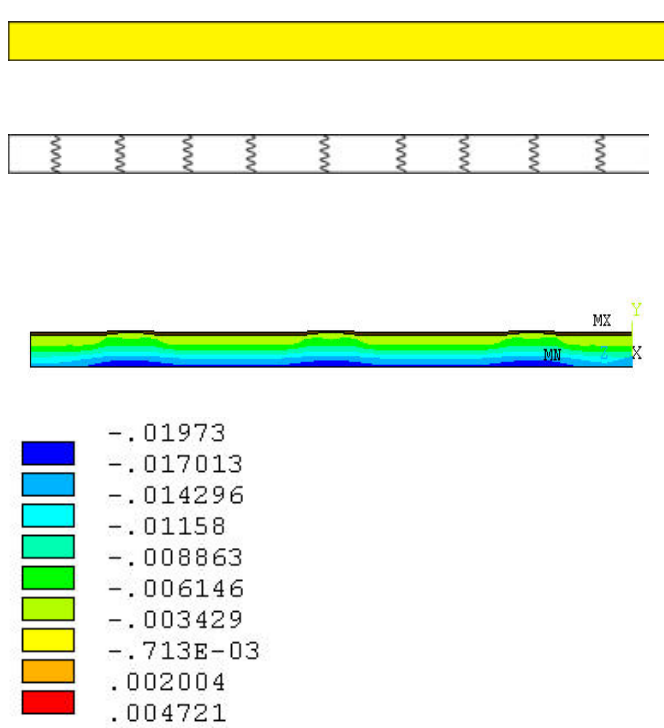

Bild 3.44: Spannungsverteilung in der Kernschicht im Auflagerbereich von Dach- und Wandelement

Die Ausbildung der oberen Deckschicht beeinflusst auch die Größe von $\mathrm{C}_{92}$. Durch die größere Steifigkeit der Deckschicht wird das Verformungsvermögen gegen Eindrücken der Dichtscheiben gemindert. Dieser Effekt ist umso größer, je näher die Verbindungsmittel im Bereich der Trapezprofilierung angeordnet sind.

Aufgrund der in diesem Abschnitt festgestellten Unterschiede werden die Dach- und Wandelemente im Rahmen der folgenden Untersuchungen unabhängig voneinander ausgewertet. 


\subsubsection{Einfluss der Pfettenauflast}

Entsprechend Gleichung (3.4) besteht ein linearer Zusammenhang zwischen der Größe des rückstellenden Moments $m_{K}$ und der auf die Pfette wirkenden Auflast. Mit der Größe des rückstellenden Momentes $m_{K}$ ändert sich der qualitative Verlauf der Momenten-Verdrehungsbeziehung wie Bild $3.26 \mathrm{zu}$ entnehmen ist. Mit den nachfolgenden Berechnungen wird untersucht, ob zudem weitere Parameter der Momenten-Verdrehungsbeziehung durch eine gesteigerte Auflast beeinflusst werden.

Die Berechnungen werden an einem Dachelement entsprechend Versuch D5/PUR mit Auflasten von $2,0 \mathrm{kN} / \mathrm{m}, 4,0 \mathrm{kN} / \mathrm{m}$ und $6,5 \mathrm{kN} / \mathrm{m}$ durchgeführt. Die ermittelten Momenten-Verdrehungsbeziehungen sind in Bild 3.45 dargestellt.

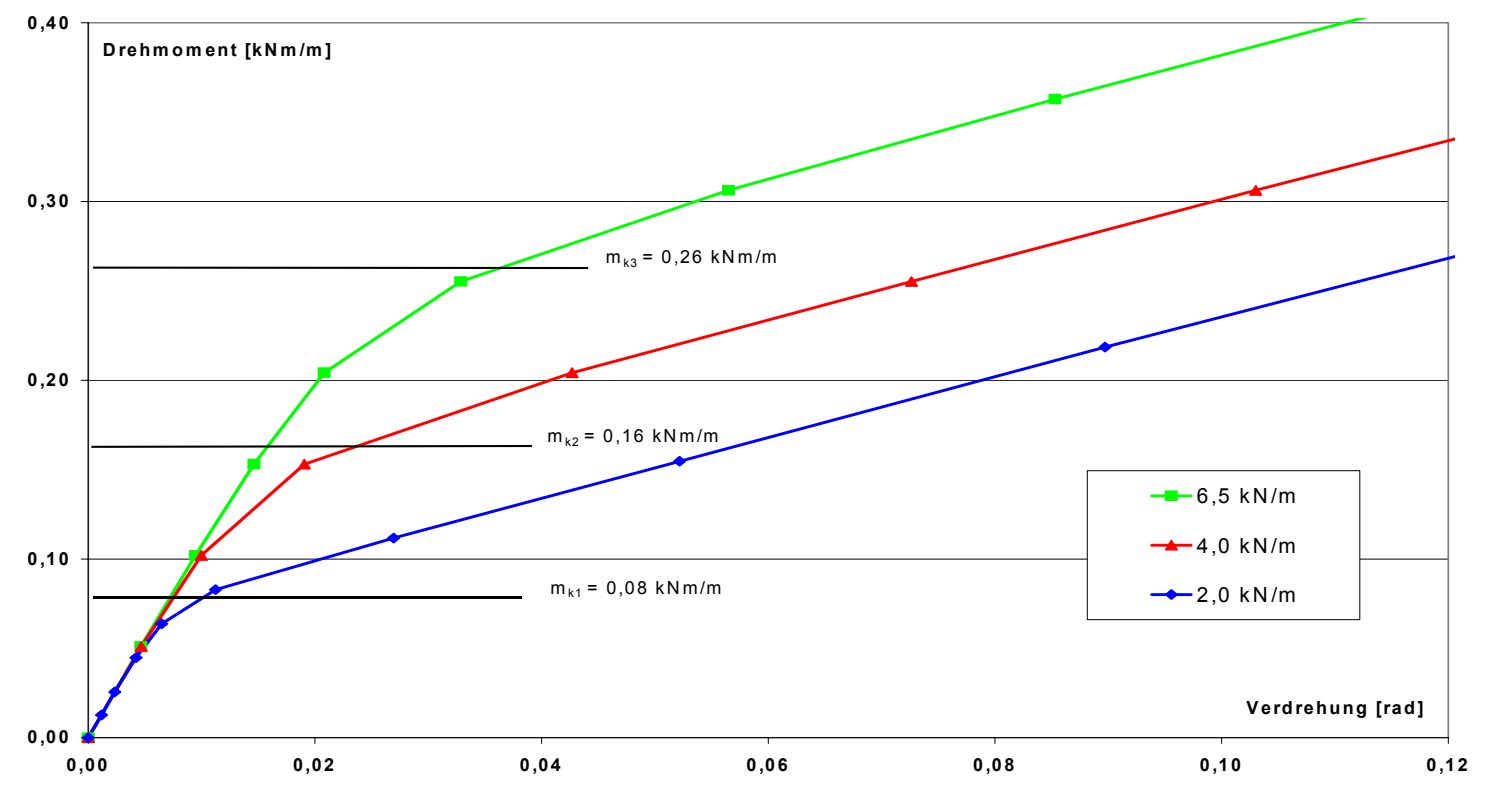

Bild 3.45: Einfluss der Pfettenauflast auf die Momenten-Verdrehungsbeziehung

Daraus ist zu erkennen, dass im Bereich kleiner Verdrehungen die Anfangssteigung nicht von der Auflast abhängt. Entsprechend den Ausführungen in Abschnitt 3.1.7 tritt die Steifigkeitsänderung bei etwa $2 / 3$ des jeweiligen Kontaktmomentes $m_{K}$ ein. Nach Überschreiten des Kontaktmomentes $m_{K}$ verlaufen die Kurven für die unterschiedlichen Auflasten parallel zueinander. Ein Einfluss der Auflast auf die Werte $\mathrm{C}_{\vartheta 1}$ oder $\mathrm{C}_{\vartheta 2}$ ist damit nicht zu erkennen.

\subsubsection{Einfluss der Elementstützweite}

Die Stützweite von Sandwichelementen wird durch eine statische Berechnung unter Berücksichtigung von Lastannahmen bestimmt und im Regelfall durch die aufnehmbare Knitterspannung der oberen Deckschicht beschränkt.

Zur Ermittlung des Einflusses unterschiedlicher Elementstützweiten wurde das bereits im Abschnitt 3.2.5.3 betrachtete Dachelement mit Stützweiten Is von $1,5 \mathrm{~m}$, $2,5 \mathrm{~m}$ und 3,5 $\mathrm{m}$ und einer Pfettenbelastung von 6,5 kN/m untersucht. Die Stützweite von 3,5 m stellt dabei hinsichtlich des Bemessungskriteriums Knitterspannung unter 
der gewählten Belastung von $6,5 \mathrm{kN} / \mathrm{m}$ den Grenzwert der Tragfähigkeit dar. Die ermittelten Momenten-Verdrehungsbeziehungen für die drei aufgeführten Stützweiten sind in Bild 3.46 dargestellt:

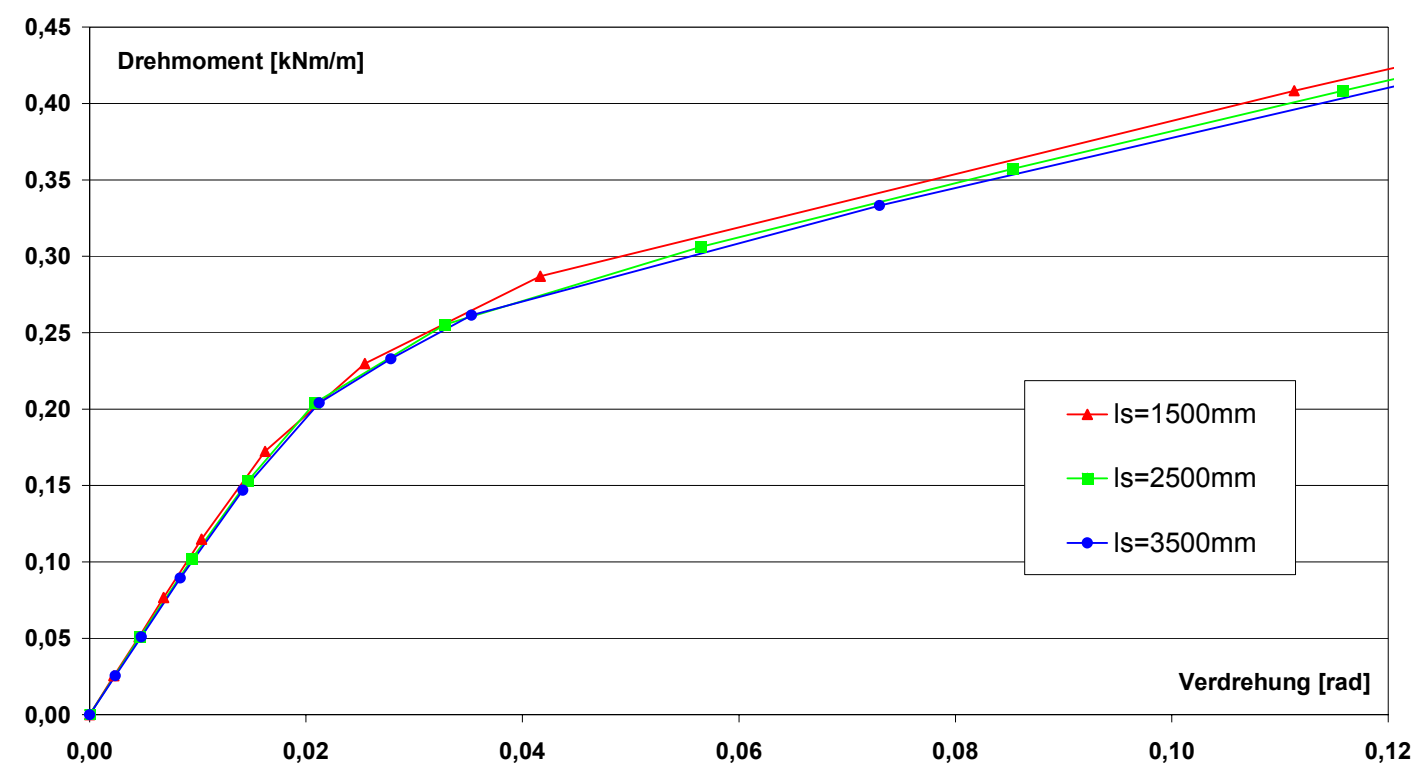

Bild 3.46: Einfluss der Elementstützweite $I_{s}$ auf die Momenten-Verdrehungsbeziehung

Der Verlauf aller drei Kurven ist fast identisch. Insbesondere haben die MomentenVerdrehungsbeziehungen sowohl im Anfangsbereich als auch im Bereich von Verdrehungen $>0,04$ rad identische Steigungen. Die Bereiche, in denen die Kurven nicht exakt deckungsgleich verlaufen, sind auf den Anteil $\mathrm{C}_{9 \mathrm{M}}$ zurückzuführen, der linear von der Stützweite abhängt. Die bei dieser Untersuchung ermittelten Abweichungen betragen maximal $4 \%$ und sind vernachlässigbar. Damit kann die Elementstützweite als maßgebender Parameter hinsichtlich der Drehstabilisierung ausgeschlossen werden.

Diese Feststellung wird durch die Ergebnisse der experimentellen Untersuchungen bestätigt. Die Versuche D1/PUR bis D6/PUR wurden paarweise mit variierter Stützweite durchgeführt. Die jeweiligen Versuchsreihen mit 2,5 m Stützweite und 3,5 m Stützweite zeigten einen nahezu gleichwertigen Verlauf in den MomentenVerdrehungsbeziehungen. Insbesondere konnten bei den aus diesen Versuchen ermittelten und in Tabelle 3.2 aufgeführten Werten $c_{91}$ und $c_{92}$ unter Berücksichtigung der versuchstypischen Streuung keine nennenswerte Unterschiede festgestellt werden.

\subsubsection{Einfluss des Elastizitätsmoduls der Sandwich-Kernschicht}

Die mechanischen Eigenschaften einer Kernschicht aus Polyurethan-Hartschaum werden maßgeblich durch die Schaumrezeptur und den Herstellprozess bestimmt. Die üblichen Werte des Elastizitätsmoduls $\mathrm{E}_{S}$, der entsprechend den allgemeinen bauaufsichtlichen Zulassungen als Mittelwert aus Zug- und Druckmodul der Kernschicht berechnet wird, liegen etwa im Bereich zwischen 2,0 und 6,0 N/mm². Dieser Streubereich wird den nachfolgenden Berechnungen zugrunde gelegt. 
Unter Berücksichtigung von zwei unterschiedlichen Obergurtbreiten des Pfettenprofils ( $b_{\mathrm{OG} 1}=82 \mathrm{~mm}, \mathrm{~b}_{\mathrm{OG} 2}=60 \mathrm{~mm}$ ) wurde ein dem Versuch W1/PUR entsprechendes Wandelement mit $40 \mathrm{~mm}$ dicker Kernschicht und einer Pfettenauflast von 3,0 kN/m numerisch untersucht. Die resultierende Momenten-Verdrehungsbeziehung für $\mathrm{b}_{\mathrm{OG} 1}=82 \mathrm{~mm}$ ist in Bild 3.47 dargestellt, die für $\mathrm{b}_{\mathrm{OG} 2}=60 \mathrm{~mm}$ in Bild 3.48 .

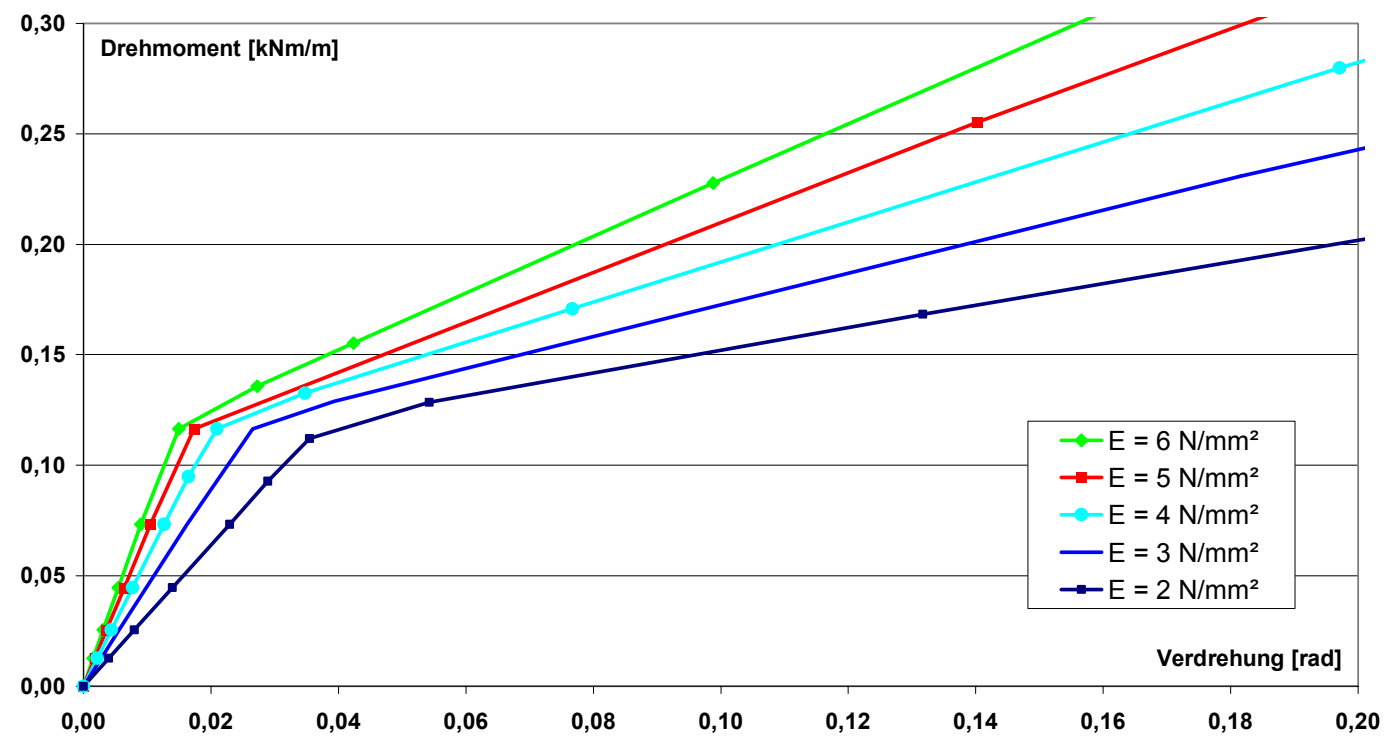

Bild 3.47: Einfluss des Elastizitätsmoduls $E_{S}$ auf die Momenten-Verdrehungsbeziehung $\left(b_{O G 1}=82 m m\right)$

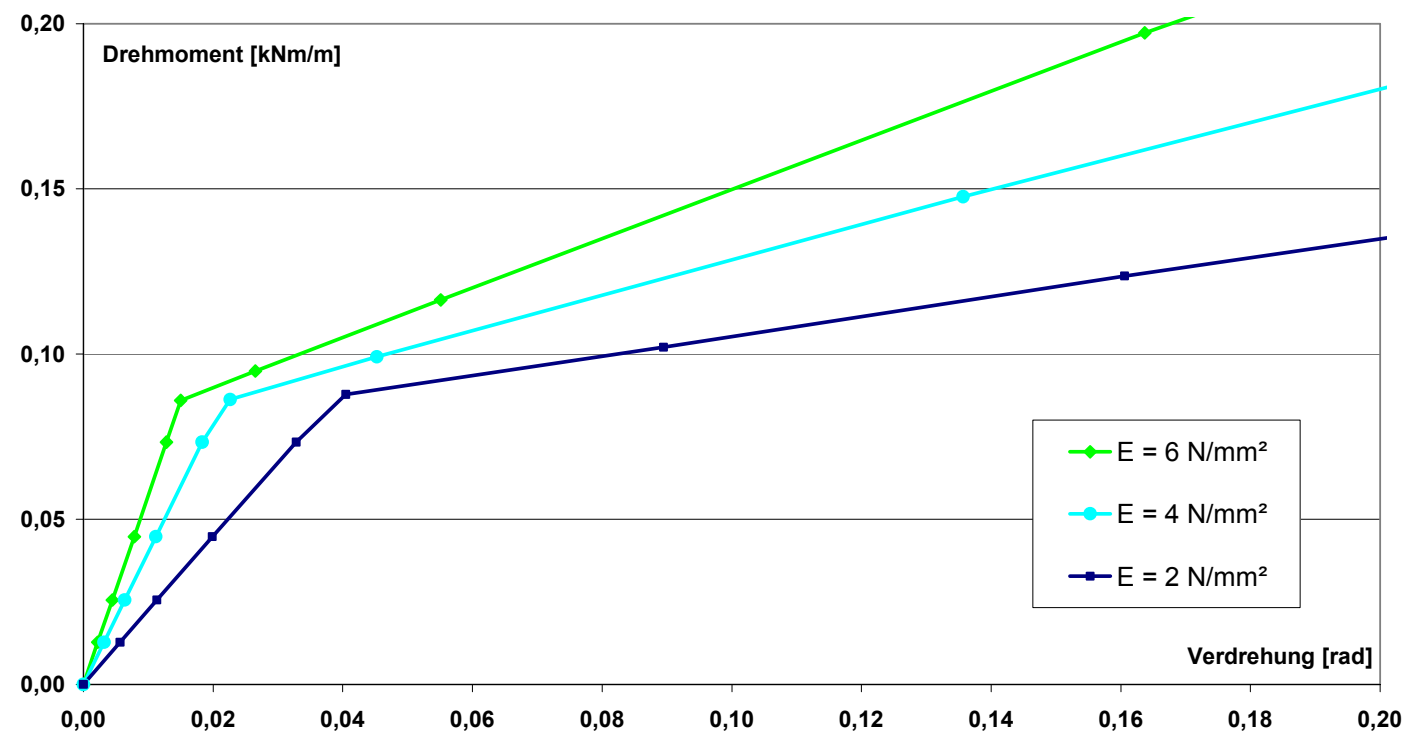

Bild 3.48: Einfluss des Elastizitätsmoduls $E_{S}$ auf die Momenten-Verdrehungsbeziehung ( $\left.b_{\mathrm{OG} 2}=60 \mathrm{~mm}\right)$

Beide Diagramme zeigen, dass sowohl $\mathbf{c}_{91}$ als auch $\mathbf{c}_{\vartheta 2}$ mit dem Elastizitätsmodul deutlich zunehmen. Das liegt daran, dass mit zunehmendem Elastizitätsmodul auch der Widerstand der Kernschicht gegen Eindrücken der Schraubenköpfe und der Pfette größer wird. Die aus den Diagrammen ermittelten Werte $c_{\vartheta 1}$ und $c_{\vartheta 2}$ sind in Bild 3.49 dargestellt und zeigen eine lineare Abhängigkeit von dem Elastizitätsmodul. 
Das Kontaktmoment wird dagegen erwartungsgemäß nicht durch den Elastizitätsmodul beeinflusst.

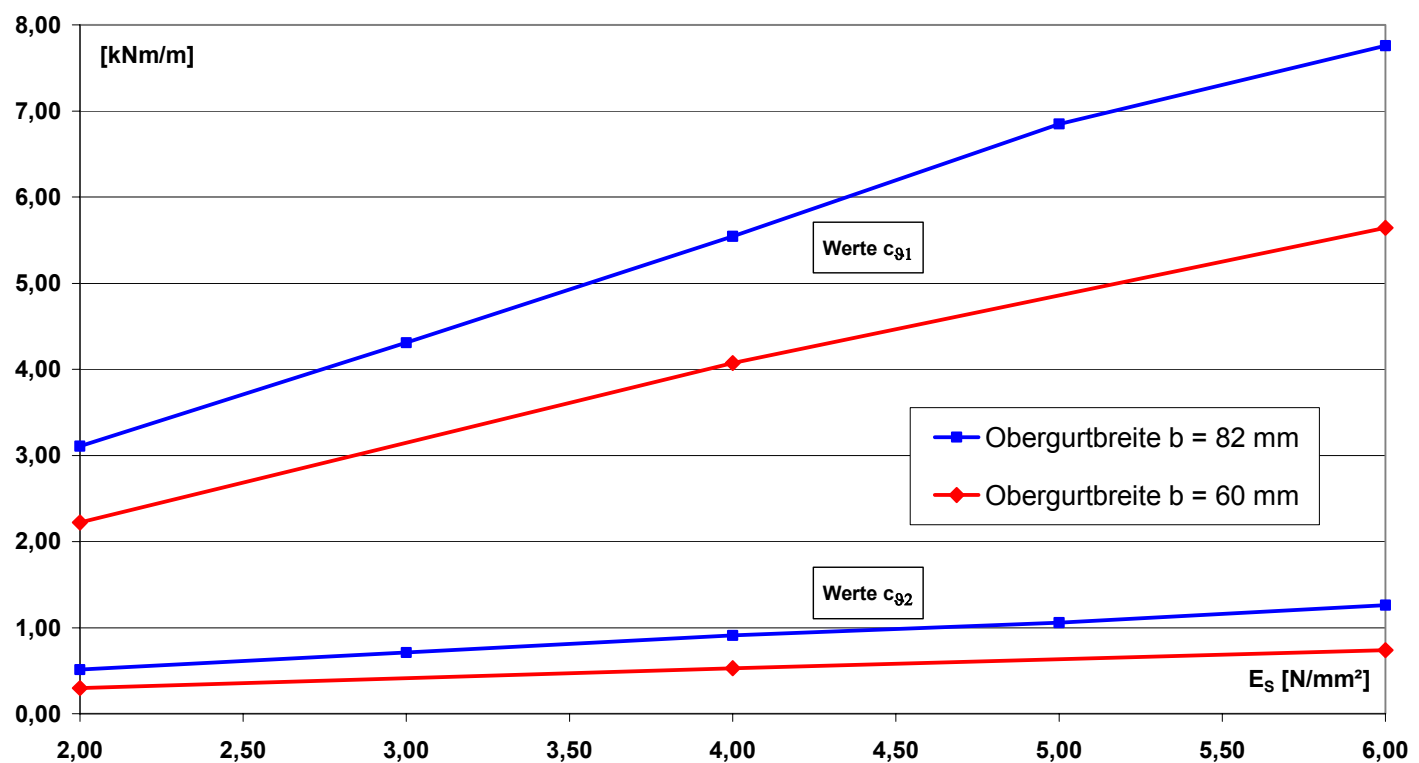

Bild 3.49: Werte $c_{\vartheta 1}$ und $c_{\vartheta 2}$ in Abhängigkeit des Elastizitätsmoduls $E_{S}$

\subsubsection{Einfluss der Elementdicke}

Die Dicke der in einem Bauwerk eingesetzten Sandwichelemente hängt im Regelfall von den bauphysikalischen Anforderungen an den Wärmeschutz ab. Üblicherweise variieren die Dicken der Kernschicht zwischen $40 \mathrm{~mm}$ und $100 \mathrm{~mm}$. Für Kühlhäuser, wo Temperaturunterschiede zwischen Innen- und Außenseite der Sandwichelemente von $100 \mathrm{~K}$ auftreten können, werden auch Elemente mit Dicken bis zu $200 \mathrm{~mm}$ eingesetzt. Die in Bild 3.50 dargestellten Momenten-Verdrehungsbeziehungen ergeben sich aus numerischen Untersuchungen an Wandelementen mit Dicken zwischen $40 \mathrm{~mm}$ und $100 \mathrm{~mm}$ und einer Pfettenauflast von $3,0 \mathrm{kN} / \mathrm{m}$.

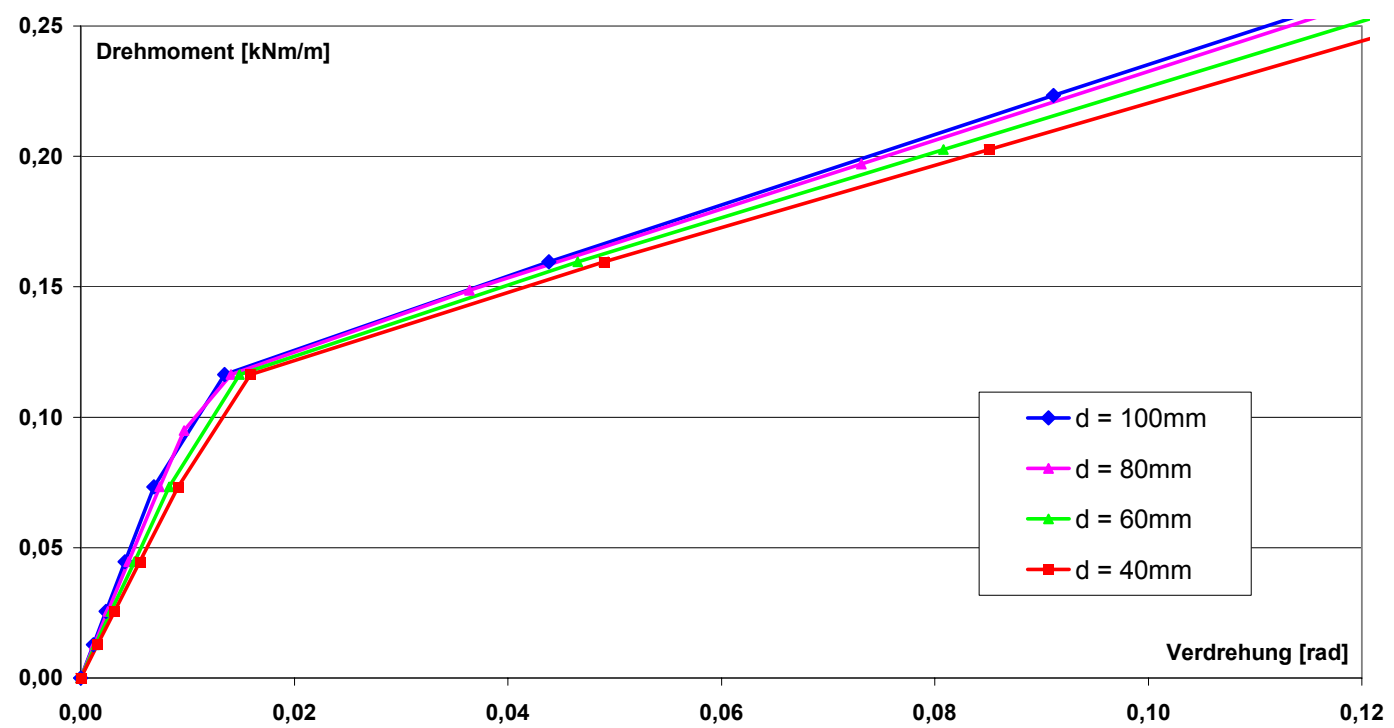

Bild 3.50: Einfluss der Elementdicke auf die Momenten-Verdrehungsbeziehung 
Aus innen ist sowohl für den Anfangsbereich als auch für den Bereich nach Überschreitung des Knickpunktes ein mit abnehmender Elementdicke flacherer Kurvenverlauf zu erkennen. Der Unterschied ist darauf zurückzuführen, dass mit größer werdender Elementdicke auch der Drehbettungsanteils $\mathrm{c}_{\vartheta \mathrm{M}}$ zunimmt. Wird dieser Anteil entsprechend Gleichung (2.7) aus der dargestellten MomentenVerdrehungsbeziehung eliminiert, ergeben sich für die untersuchten Elementdicken Anschlusssteifigkeiten $\mathrm{C}_{\vartheta \mathrm{A}}$, deren maximaler Unterschied etwa $5 \%$ beträgt. Aufgrund dieser geringfügigen Unterschiede ist der Einfluss der Elementdicke auf die Anschlusssteifigkeit von Sandwichelementen vernachlässigbar.

Der vernachlässigbare Einfluss der Elementdicke wird durch die Ergebnisse der experimentellen Untersuchungen bestätigt. Die Versuche D5/PUR und D6/PUR sowie W1/PUR und W6/PUR wurden paarweise mit unterschiedlichen Elementdicken durchgeführt. Die jeweiligen Versuchsreihen zeigten einen nahezu gleichwertigen Verlauf in den Momenten-Verdrehungsbeziehungen. Insbesondere konnten bei den aus diesen Versuchen ermittelten und in Tabelle 3.2 aufgeführten Werten $\mathbf{c}_{91}$ und $\mathbf{c}_{92}$ unter Berücksichtigung der versuchstypischen Streuung keine nennenswerte Unterschiede festgestellt werden.

\subsubsection{Einfluss der Deckschichtdicken}

Entsprechend den Angaben der allgemeinen bauaufsichtlichen Zulassung für Sandwichelemente [12] sind bei Dach- und Wandelementen Deckschichtdicken zwischen $0,40 \mathrm{~mm}$ und $1,25 \mathrm{~mm}$ zulässig. Die üblichen Werte für die schraubenkopfseitigen Deckschichten variieren zwischen $0,50 \mathrm{~mm}$ und $0,75 \mathrm{~mm}$. Deswegen wurde dieser Bereich auch den nachfolgenden Untersuchungen an einem Wandelement mit $40 \mathrm{~mm}$ dicker Kernschicht und einer Pfettenbelastung von 3,0 kN/m zugrunde gelegt. Die Variation der Blechdicke erfolgte lediglich für die äußere, schraubenkopfseitige Deckschicht, da die Blechdicke der inneren Deckschicht üblicherweise in einem engen Bereich liegt. Die Berechnung erfolgte generell unter Ansatz der Kernblechdicke $t_{K}\left(=\right.$ Nennblechdicke $t_{N}$ abzüglich 0,04 $\mathrm{mm}$ Zinkschicht) und für eine Blechdicke von $0,50 \mathrm{~mm}$ für die innere Deckschicht.

In dieser Untersuchung werden die Dichtscheiben mit einer Dicke von 2,0 mm und einem Durchmesser von $16 \mathrm{~mm}$ modelliert. Da die Dicke der Dichtscheibe um ein vielfaches größer ist als die Dicke der Deckschicht wird die Nachgiebigkeit des Anschlussbereiches maßgeblich durch die Dicke der schraubenkopfseitigen Deckschicht und den Durchmesser der Dichtscheibe bestimmt.

Aus den in Bild 3.51 dargestellten Momenten-Verdrehungsbeziehungen wird deutlich, dass sich die Variation der schraubenkopfseitigen Deckschichtdicke lediglich auf den Wert $\mathrm{C}_{92}$ auswirkt. Der Unterschied zwischen kleinster und größter untersuchter Blechdicke beträgt etwa $13 \%$. Er begründet sich dadurch, dass die Verbindungsmittel erst nach Überschreiten des Kontaktmomentes maßgeblich auf Zug beansprucht werden. Das dickere Deckblech bietet in diesem Zustand durch seine größere Eigensteifigkeit einen erhöhten Widerstand gegen ein Eindrücken der Dichtscheiben in das Element. 


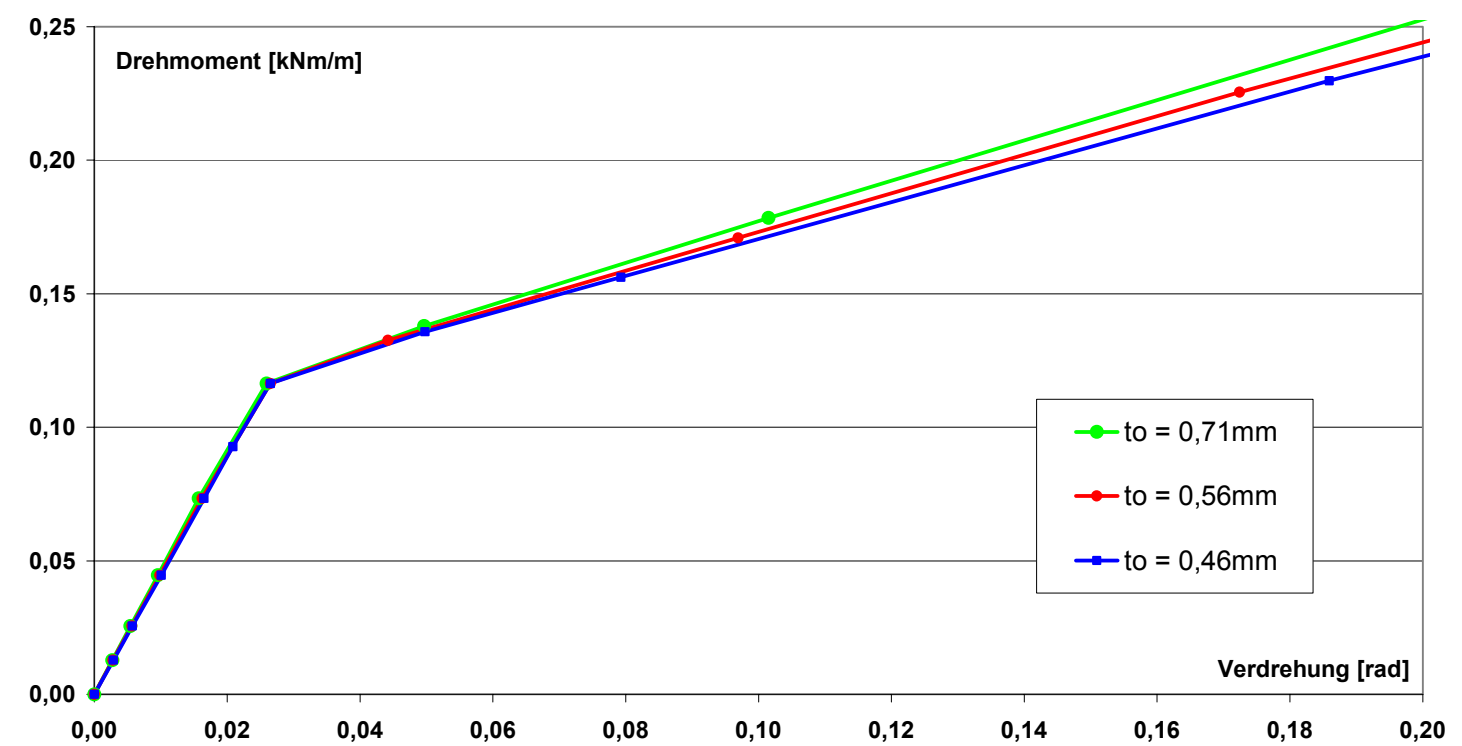

Bild 3.51: Einfluss der oberen Deckschichtdicke $t_{o}$ auf die Momenten-Verdrehungsbeziehung

\subsubsection{Einfluss der Obergurtbreite des Pfettenprofils}

Mit Ausnahme des Versuchs DZ/PUR wurden alle experimentellen Untersuchungen mit einem Pfettenprofil IPE160 durchgeführt, dessen Obergurtbreite nominell $82 \mathrm{~mm}$ betrug. In den allgemeinen bauaufsichtlichen Zulassungen für Sandwichelemente ist für Zwischenauflager jedoch auch eine Mindestbreite von $60 \mathrm{~mm}$ zulässig. Dementsprechend wurde der Einfluss der Pfettenobergurtbreite durch deren Variation mit $60 \mathrm{~mm}, 82 \mathrm{~mm}$ und $100 \mathrm{~mm}$ am Beispiel eines Wandelementes mit $40 \mathrm{~mm}$ dicker Kernschicht untersucht.

Die in Bild 3.52 dargestellten Momenten-Verdrehungsbeziehungen zeigen, dass die Werte $c_{91}$ und $c_{92}$ durch die Breite des Obergurts erheblich beeinflusst werden. Entsprechend der Definition des Kontaktmomentes wirkt sich die Obergurtbreite auf die Ordinate des Knickpunktes aus, während die zugehörige Verdrehung nahezu unverändert bleibt.

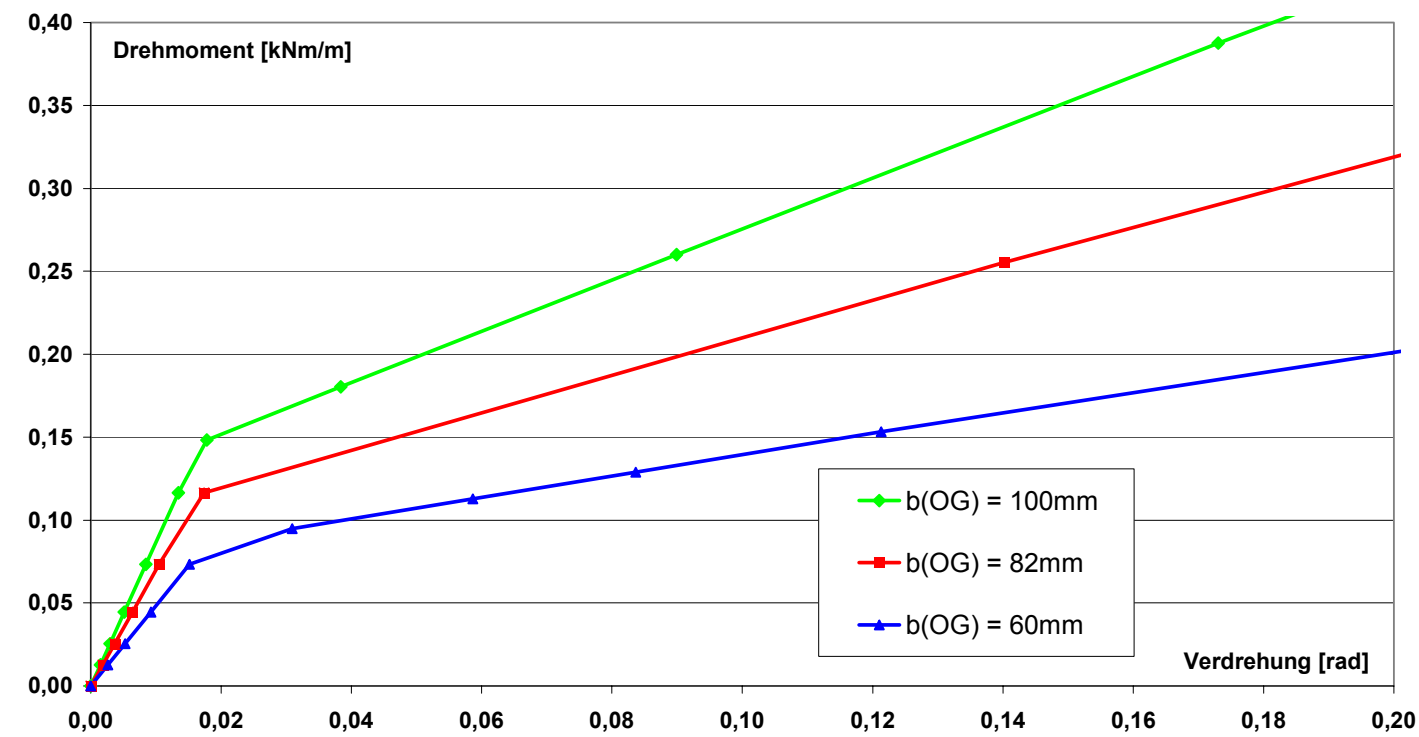

Bild 3.52: Einfluss der Obergurtbreite $b_{\mathrm{OG}}$ auf die Momenten-Verdrehungsbeziehung 
Die Auswertung der Werte $c_{91}$ und $c_{92}$ ergibt, dass beide Werte der Obergurtbreite proportional sind.

Die im Hinblick auf die Drehstabilisierung günstige Wirkung der Obergurtbreite ist darauf zurückzuführen, dass mit ihr sowohl der Hebelarm des rückstellenden Moments als auch der Hebelarm der Verschraubung größer wird.

\subsubsection{Einfluss der Verbindungsmittelanordnung}

Eine alternierende Anordnung der Verbindungsmittel zur Befestigung von Sandwichelementen auf I-Trägern wird oftmals aus ästhetischen Gründen nicht gewünscht. Stattdessen erfolgt die Verschraubung mit einseitig angeordneten Verbindungsmitteln entweder links oder rechts des Steges.

Die numerische Untersuchung der Verbindungsmittelanordnung erfolgt für ein Dachelement mit $40 \mathrm{~mm}$ dicker Kernschicht in Verbindung mit einem Profil IPE160 und einer Pfettenauflast von 3,1 kN/m. Bei einer stets gleichen Pfettendrehrichtung entgegen dem Uhrzeigersinn werden folgende 3 Fälle unterschieden:

- Anordnung der Verbindungsmittel rechts vom Steg (ungünstiger Fall)

- Anordnung der Verbindungsmittel links vom Steg (günstiger Fall)

- alternierende Anordnung der Verbindungsmittel

Die Berechnungsergebnisse in Bild 3.53 zeigen für die drei möglichen Fälle, dass die Anordnung der Verbindungsmittel lediglich den Wert $\mathbf{c}_{\vartheta 2}$ der MomentenVerdrehungsbeziehung beeinflusst. Der Kurvenverlauf bis zum Erreichen des Kontaktmomentes ist für alle drei Fälle identisch.

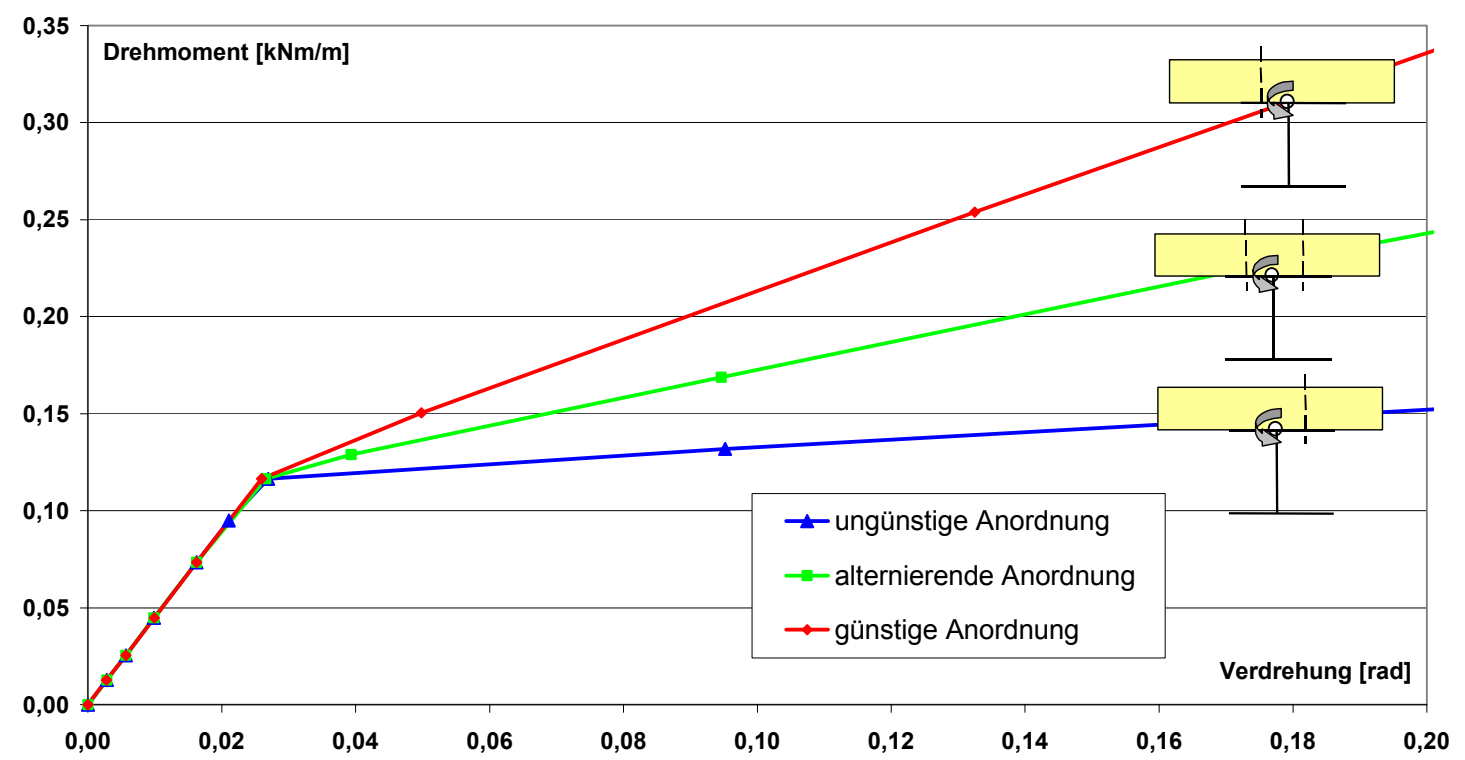

Bild 3.53: Einfluss der Verbindungsmittelanordnung auf die Momenten-Verdrehungsbeziehung

Bestätigt werden diese Ergebnisse durch die Versuche W2/PUR und W3/PUR, in denen zum einen eine alternierende zum anderen eine einseitige Verbindungsmittel- 
anordnung untersucht wurde. Unter Berücksichtigung der versuchstypischen Streuung zeigten beide Versuche etwa gleich große Werte für $\mathbf{c}_{91}$. Zudem zeigt sowohl die experimentelle als auch die numerische Untersuchung, dass die Größe des Werts $\mathrm{C}_{\vartheta 2}$ bei alternierender Anordnung in guter Näherung als Mittelwert aus günstiger und ungünstiger Anordnung berechnet werden kann.

\subsubsection{Einfluss einer geänderten Profilform}

Außer den bisher untersuchten I-Trägern werden unter anderem auch dünnwandige Bauteile mit Z-Querschnitt als Pfettenprofile oder Wandriegel verwendet. Die Befestigung erfolgt in diesen Fällen stets in der Mitte des Gurtes, auf dem das Sandwichelement aufliegt.

Nachfolgende Berechnungen beruhen auf der Annahme, dass sich die Drehachse des Profils entsprechend den Untersuchungen zuvor weiterhin im Schnittpunkt von Steg und oberem Flansch befindet. Die Berechnungen wurden an einem Z-Profil mit $160 \mathrm{~mm}$ Höhe und $60 \mathrm{~mm}$ Flanschbreite in Kombination mit einem Wandelement mit $40 \mathrm{~mm}$ dicker Kernschicht und variabler Pfettenauflast durchgeführt. Damit sich Effekte aus der Anschlussnachgiebigkeit mit Nachgiebigkeiten aus der Profilverformung nicht überlagern, wurde das Profil mit einer Blechdicke von 5,0 mm modelliert. Die Profilverformung wird damit vernachlässigbar.

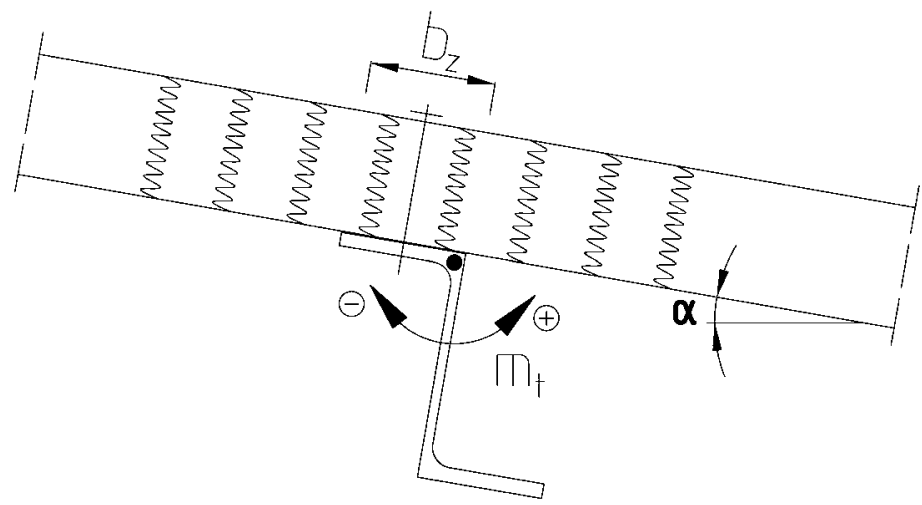

Bild 3.54: Darstellung eines Z-Profils im Einbauzustand

Die Untersuchung muss hinsichtlich der Pfettendrehrichtung unterschieden werden. Bei Betrachtung von Bild 3.54 wird deutlich, dass eine Profilverdrehung im Uhrzeigersinn (negativ) das Steifigkeitsverhalten günstig beeinflusst, da der an der Sandwichunterseite anliegende Schenkel abgestützt wird. Eine Verdrehung entgegen dem Uhrzeigersinn (positiv) wird dagegen nur durch die Verbindungsmittel behindert. Dieser Sachverhalt wird sowohl durch die in Bild 3.55 dargestellte MomentenVerdrehungsbeziehung als auch durch die Ergebnisse des Versuchs DZ/PUR bestätigt. In beiden Fällen ist das unterschiedliche Steifigkeitsverhalten in Abhängigkeit der Drehrichtung deutlich zu erkennen. 


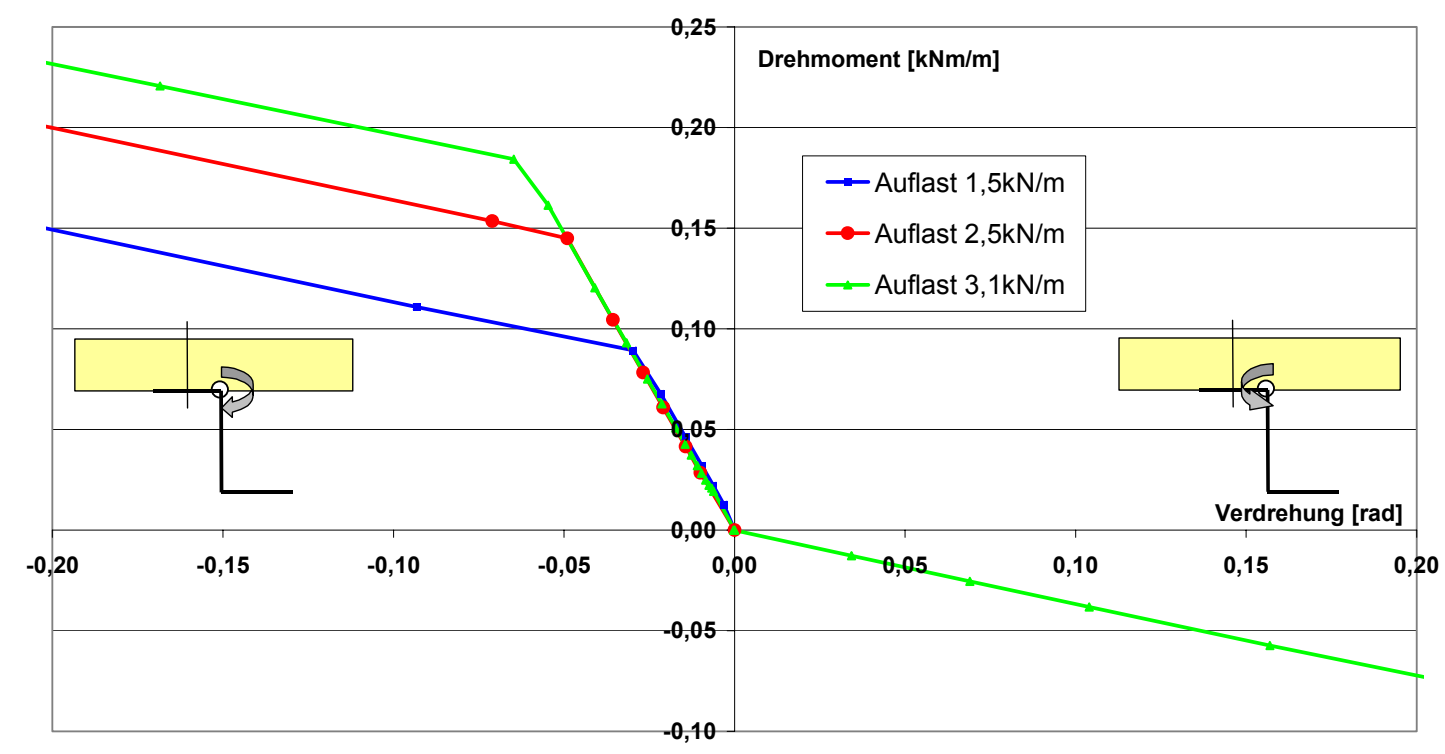

Bild 3.55: Momenten-Verdrehungsbeziehung für Z-Profil Z160x60

Der bilineare Kurvenverlauf bei negativer Drehrichtung entspricht qualitativ dem, der in den Abschnitten zuvor für I-Profile ermittelt wurde. Die beim Z-Profil im Vergleich zum I-Profil fehlende Obergurthälfte macht sich bei diesem Drehsinn nicht bemerkbar. Der Übergang von $\mathrm{c}_{91} \mathrm{zu}_{\mathrm{C}_{92}}$ tritt analog zu Gleichung (3.4) bei

$$
m_{K}=q \cdot b_{Z}
$$

ein. An die Stelle des Hebelarms von halber Obergurtbreite des I-Profils tritt in diesem Fall die Breite $b_{z}$ des an der Elementunterseite anliegenden Schenkels des ZProfils. Für den Fall des Z-Profils mit negativer Drehrichtung liegt damit eine Analogie zum Fall des I-Profils mit ungünstiger Anordnung der Verbindungsmittel aus 3.2.5.9 vor.

Bei positiver Drehrichtung liegt bereits von Anfang an ein flacher, linearer Kurvenverlauf vor, der maßgeblich durch die Nachgiebigkeit des Anschlussbereiches bestimmt wird. Die Größenordnung der Kurvensteigung entspricht dabei in etwa der Steigung des bilinearen Kurvenverlaufs nach Überschreiten des Kontaktmomentes bei negativer Drehrichtung. 


\subsection{Auswertung der Ergebnisse}

\subsubsection{Definition von Einflussfaktoren}

Im Hinblick auf die nachfolgende statistische Auswertung der unter Abschnitt 3.1.7 ermittelten Werte $\mathbf{c}_{91}$ und $\mathbf{c}_{92}$ müssen geeignete Populationen definiert werden. Dazu muss bekannt sein, durch welche Parameter die Werte $c_{\vartheta 1}$ und $c_{\vartheta 2}$ beeinflusst werden.

Die Ergebnisse der experimentellen Untersuchungen für Dach- und Wandelemente werden entsprechend den Erkenntnissen aus Abschnitt 3.2.5.2 getrennt statistisch ausgewertet. Die Berücksichtigung des Kriecheinflusses kann mit diesem Vorgehen direkt auf die ausgewerteten Ergebnisse der Dachelemente angewandt werden.

Nach den Ergebnissen der experimentellen und numerischen Untersuchungen wird der Wert $\mathbf{c}_{91}$ maßgeblich durch die Breite des Pfettenobergurts sowie den Elastizitätsmodul der Sandwichkernschicht bestimmt. Der Wert $\mathrm{c}_{\vartheta 2}$ wird zusätzlich von der Ausbildung und Nachgiebigkeit des Anschlussbereiches beeinflusst. Dazu gehören neben der Anzahl und Anordnung der Verbindungsmittel auch die Größe der Dichtscheiben sowie die Dicke der oberen Deckschicht. Die Größe des Kontaktmoments wird entsprechend Gleichung (3.4) durch die Pfettenauflast und die Breite des Pfettenobergurts bestimmt.

Aufgrund der zahlreichen Parameter und Kombinationsmöglichkeiten müssen Vereinfachungen getroffen werden. Dazu muss sichergestellt werden, dass die Vereinfachungen die wirklichen Zustände nicht überschätzen. So wurden zum Beispiel alle Versuche des Abschnitts 3.1 mit der erforderlichen Mindestanzahl von zwei Verbindungsmitteln pro Element durchgeführt. Dies stellt - mit Ausnahme der verdeckten Befestigungsart - gleichzeitig den baupraktischen Regelfall der Elementbefestigung dar.

Der Einfluss der Dichtscheibengröße wird aus vereinfachenden Gründen vernachlässigt. Wie der Vergleich der Versuche W4/PUR und W5/PUR zeigt, ergeben sich für die Durchmesser der Dichtscheiben von $16 \mathrm{~mm}$ und $19 \mathrm{~mm}$ nur geringfügige Unterschiede hinsichtlich der Werte $\mathrm{c}_{\vartheta 2}$. Auf eine getrennte Berücksichtigung dieses Parameters für die nachfolgende statistische Auswertung wurde deshalb verzichtet.

\subsubsection{Statistische Auswertung der Versuchsergebnisse hinsichtlich $\mathbf{c}_{91}$}

\subsubsection{Elemente mit Kernschicht aus Polyurethan-Hartschaum}

Zur statistischen Auswertung der Werte $c_{91}$ werden die experimentellen Untersuchungen an den Dach- und Wandelementen mit Kernschicht aus PolyurethanHartschaum zu jeweils einer Population zusammengefasst. Da die betreffenden Versuche D1/PUR bis D7/PUR und W1/PUR bis W6/PUR jeweils mit dem gleichen Pfettentyp durchgeführt wurden, verbleibt als maßgebender Parameter hinsichtlich des Werts $\mathbf{C}_{91}$ lediglich der Elastizitätsmodul der Sandwichkernschicht. 
Entsprechend den Ausführungen in Abschnitt 3.2.5.5 besteht ein linearer Zusammenhang zwischen dem Elastizitätsmodul der Kernschicht und dem Wert $\mathbf{c}_{91}$. Die statistische Auswertung der ermittelten Werte $c_{91} \_$pos und $c_{91}$ neg (vgl. Tabelle 3.2 und Tabelle 3.3) erfolgt deswegen mit Bezug auf den Elastizitätsmodul. Aus diesen Werten werden für jede Population der Mittelwert $\bar{x}$ sowie die Quotienten $x_{i} / \bar{x}$ berechnet. Mit dem Variationskoeffizienten s ergeben sich die mit einer Aussagewahrscheinlichkeit von $75 \%$ verbundenen 5\%-Fraktilwerte entsprechend Tabelle A5.1 des Anhangs A5 zu

$$
c_{\vartheta 1}=\left(1-k_{s} \cdot s\right) \cdot \bar{x} \cdot E_{s}
$$

mit $\mathrm{k}_{\mathrm{S}}$ entsprechend ISO12491. Für Elemente mit Kernschicht aus PolyurethanHartschaum ergeben sich daraus die in Tabelle 3.11 angegebenen Werte $c_{91}$. Der ebenfalls in Tabelle 3.11 angegebene Gültigkeitsbereich erfasst die üblichen Werte des Elastizitätsmoduls und ist durch die Untersuchungen aus Abschnitt 3.2.5.5 abgedeckt.

\begin{tabular}{|c|c|c|}
\hline & $\mathrm{c}_{91}[\mathrm{kNm} / \mathrm{m}]$ & Gültigkeitsbereich \\
\hline PUR-Wandelement & $1,20 \cdot E_{S}$ & \multirow{2}{*}{$\mathrm{E}_{\mathrm{S}} \in[2,0 ; 6,0] \mathrm{in}\left[\mathrm{N} / \mathrm{mm}^{2}\right]$} \\
\hline PUR-Dachelement & $1,60 \cdot E_{S}$ & \\
\hline
\end{tabular}

Tabelle 3.11: statistisch ermittelte Werte $c_{91}$ für PUR-Dach- und Wandelemente

\subsubsection{Elemente mit Kernschicht aus Mineralfasern}

Die statistische Auswertung der Versuche an Elementen mit Kernschicht aus Mineralfasern (Versuche D1/MW und W2/MW) hinsichtlich der Werte $\mathrm{c}_{91}$ erfolgt analog zu Abschnitt 3.3.2.1. Aufgrund der irreversiblen Schädigung der Mineralfasern beim Versuch W2/MW (vgl. Abschnitt 3.1.4.3) wurden die Werte $c_{\vartheta 1}$ der ersten Laststufe auf das Niveau der übrigen Laststufen abgemindert.

Die experimentellen Untersuchungen an den Dach- und Wandelementen mit Kernschicht aus Mineralfasern werden zu jeweils einer Population zusammengefasst und entsprechend Tabelle A5.3 des Anhangs A5 mit Bezug auf den Elastizitätsmodul getrennt statistisch ausgewertet.

Für die Elemente mit Kernschicht aus Mineralfasern ergeben sich daraus die in Tabelle 3.12 angegebenen Werte $\mathrm{c}_{\vartheta 1}$.

\begin{tabular}{|c|c|c|}
\hline & $\mathrm{c}_{91}[\mathrm{kNm} / \mathrm{m}]$ & Gültigkeitsbereich \\
\hline MW-Wandelement & $0,48 \cdot E_{S}$ & \multirow{2}{*}{$\mathrm{E}_{\mathrm{S}} \in[2,0 ; 6,0] \mathrm{in}\left[\mathrm{N} / \mathrm{mm}^{2}\right]$} \\
\hline MW-Dachelement & $0,69 \cdot E_{S}$ & \\
\hline
\end{tabular}

Tabelle 3.12: statistisch ermittelte Werte $c_{91}$ für MW-Dach- und Wandelemente

Die Anforderung an den Mindestwert des Elastizitätsmoduls $E_{s}$ variiert für Sandwichelemente nach [9] je nach Elementdicke zwischen $3,9 \mathrm{~N} / \mathrm{mm}^{2}$ und $6,2 \mathrm{~N} / \mathrm{mm}^{2}$. Die im Rahmen der Untersuchungen nach Abschnitt 3.1.6.3 ermittelten Werte für $E_{s}$ lagen deutlich über den jeweiligen Mindestwerten. Aufgrund der verhältnismäßig ge- 
ringen Versuchsanzahl wird das anwendbare Intervall des Elastizitätsmoduls $E_{s}$ bei den Mineralfaser-Elementen durch einen Maximalwert von 6,0 N/mm² beschränkt.

\subsubsection{Statistische Auswertung der Versuchsergebnisse hinsichtlich $\mathbf{c}_{\vartheta 2}$}

\subsubsection{Elemente mit Kernschicht aus Polyurethan-Hartschaum}

Zur statistischen Auswertung der Werte $\mathrm{c}_{92}$ werden die experimentellen Untersuchungen an den Dach- (Versuche D1/PUR bis D6/PUR) und Wandelementen (Versuche W1/PUR, W2/PUR, W4/PUR bis W6/PUR) zu jeweils einer Population zusammengefasst. Die Verbindungsmittel waren in allen Fällen alternierend angeordnet.

Die statistische Auswertung der ermittelten Werte $c_{92}$ pos und $c_{\vartheta 2}$ neg (vgl. Tabelle 3.2 und Tabelle 3.3) erfolgt entsprechend den Ausführungen der Äbschnitte 3.2.5.5 und 3.2.5.7 mit Bezug auf die Größe des Elastizitätsmoduls und die Kernblechdicke der oberen Deckschicht. Aus den bezogenen Werten werden für jede Population der Mittelwert $\bar{x}$ sowie die Quotienten $x_{i} / \bar{x}$ berechnet. Mit dem Variationskoeffizienten s ergeben sich die mit einer Aussagewahrscheinlichkeit von $75 \%$ verbundenen $5 \%$ Fraktilwerte entsprechend Tabelle A5.2 des Anhangs A5 zu

$$
c_{\vartheta 2}=\left(1-k_{s} \cdot s\right) \cdot \bar{x} \cdot t_{K} \cdot E_{s}
$$

mit $\mathrm{k}_{\mathrm{S}}$ entsprechend ISO12491. Für Elemente mit Kernschicht aus PolyurethanHartschaum ergeben sich daraus die in Tabelle 3.13 angegebenen Werte $\mathbf{c}_{\vartheta 2}$.

\begin{tabular}{|c|c|c|}
\hline & $\mathrm{c}_{92}[\mathrm{kNm} / \mathrm{m}]$ & Gültigkeitsbereich \\
\hline PUR-Wandelement & $0,38 \cdot t_{K} \cdot E_{S}$ & \multirow{2}{*}{$\mathrm{E}_{\mathrm{S}} \in[2,0 ; 6,0] \mathrm{in}\left[\mathrm{N} / \mathrm{mm}^{2}\right]$} \\
\cline { 1 - 1 } & $0,44 \cdot t_{K} \cdot E_{S}$ & $\mathrm{t}_{\mathrm{K}} \in[0,42 ; 0,67] \mathrm{in}[\mathrm{mm}]$ \\
\hline
\end{tabular}

Tabelle 3.13: statistisch ermittelte Werte $c_{\vartheta 2}$ für PUR-Dach- und Wandelemente

Der große Wert für die Standardabweichung ist darauf zurückzuführen, dass neben der üblichen Streuung der mechanischen Kennwerte der Sandwichelemente Einflüsse bei der Elementverschraubung dazu führen, dass identische Versuchsbedingungen kaum realisierbar sind. Hierzu sind insbesondere Lageabweichungen der Verschraubung im Pfettenobergurt, Einflüsse beim Vorbohren der gewindefurchenden Schrauben sowie Unterschiede in den Schraubenanzugsmomenten zu nennen.

\subsubsection{Elemente mit Kernschicht aus Mineralfasern}

Die statistische Auswertung der Versuche D1/MW und W2/MW hinsichtlich der Werte $\mathrm{c}_{\vartheta 2}$ erfolgt analog zu Abschnitt 3.3.3.1. Dazu wurden die experimentellen Untersuchungen an den Dach- und Wandelementen zu jeweils einer Population zusammengefasst und entsprechend Tabelle A5.4 des Anhangs A5 mit Bezug auf den Elastizitätsmodul und die Kernblechdicke getrennt statistisch ausgewertet. 
Für die Elemente mit Kernschicht aus Mineralfasern ergeben sich daraus die in Tabelle 3.14 angegebenen Werte $\mathrm{c}_{\vartheta 2}$.

\begin{tabular}{|c|c|c|}
\hline & $c_{92}[\mathrm{kNm} / \mathrm{m}]$ & Gültigkeitsbereich \\
\hline MW-Wandelement & $0,16 \cdot t_{K} \cdot E_{S}$ & \multirow{2}{*}{$E_{S} \in[2,0 ; 6,0] \mathrm{in}\left[\mathrm{N} / \mathrm{mm}^{2}\right]$} \\
$t_{\mathrm{K}} \in[0,42 ; 0,67] \mathrm{in}[\mathrm{mm}]$
\end{tabular}

Tabelle 3.14: statistisch ermittelte Werte $c_{\vartheta 2}$ für MW-Dach- und Wandelemente

Entsprechend den Ausführungen aus Abschnitt 3.3.2.2 wird aufgrund der verhältnismäßig geringen Versuchsanzahl das anwendbare Intervall des Elastizitätsmoduls $E_{s}$ bei den Mineralfaser-Elementen durch einen Maximalwert von $6,0 \mathrm{~N} / \mathrm{mm}^{2}$ beschränkt.

\subsubsection{Berücksichtigung des Kriecheinflusses bei PUR-Sandwichelementen}

Die Bestimmung des Kriecheinflusses erfolgt durch Auswertung und Vergleich der unter Abschnitt 3.1.4.4 beschriebenen Versuche D7a/PUR mit festen Verbindungsmitteln und D7b/PUR mit gelösten Verbindungsmitteln. Die aus den jeweiligen Momenten-Verdrehungsbeziehungen ermittelten Werte $c_{91}$ und $c_{92}$ sind in Tabelle 3.15 zusammen mit den jeweiligen Verhältniswerten dargestellt.

\begin{tabular}{|c|c|c|c|c|}
\hline & \multicolumn{2}{|c|}{ Werte $\mathrm{c}_{91}[\mathrm{kNm} / \mathrm{m}]$} & \multicolumn{2}{c|}{ Werte $\mathrm{c}_{92}[\mathrm{kNm} / \mathrm{m}]$} \\
\hline Drehrichtung & positiv & negativ & positiv & negativ \\
\hline Versuch D7a/PUR & $7,17 / 8,85 / 9,49$ & $7,50 / 8,95 / 9,89$ & $2,01 / 2,01 / 1,89$ & $2,53 / 2,47 / 2,29$ \\
\hline Versuch D7b/PUR & $6,19 / 8,26 / 8,95$ & $6,44 / 8,26 / 9,36$ & $1,01 / 0,98 / 1,22$ & $0,73 / 0,80 / 0,94$ \\
\hline Verhältniswerte & $0,86 / 0,93 / 0,94$ & $0,86 / 0,92 / 0,95$ & $0,50 / 0,49 / 0,65$ & $0,29 / 0,32 / 0,41$ \\
\hline
\end{tabular}

Tabelle 3.15: Zusammenstellung der Werte $c_{\vartheta 1}$ und $c_{\vartheta 2}$ aus den Versuchen zum Kriecheinfluss

Die Verhältniswerte zeigen, dass sich der simulierte Kriecheinfluss durch Lösen der Verbindungsmittel erwartungsgemäß deutlich auf die Werte $\mathbf{c}_{\vartheta 2}$ auswirkt. Die Abminderung aufgrund der fehlenden Kraftübertragung von den Schraubenköpfen auf die Kernschicht beträgt dabei bis zu $70 \%$. Mit dieser enormen Abminderung ist eine Beeinflussung der Werte $\mathrm{c}_{\vartheta 1}$ nicht auszuschließen, was die in Tabelle 3.15 aufgeführten Verhältniswerte für diese Werte bestätigen.

Für die Bewertung dieser Untersuchung ist zu berücksichtigen, dass das Lösen der Verbindungsmittel um zwei Umdrehungen und der damit verbundene vollständige Verlust der Kontaktwirkung den ungünstigsten Fall darstellt, der in dieser Ausprägung im Bauwerk nicht eintreten wird. Viel mehr wird sich die in Bild 3.21 skizzierte Verformung des Auflagerbereichs als eine Relaxation auf die Verbindungsmittel auswirken.

Im Hinblick auf die Drehstabilisierung ist vor allem das Steifigkeitsverhalten bis zum Erreichen des Kontaktmomentes maßgebend. Deshalb wird zur Berücksichtigung des Kriecheinflusses bei Polyurethan-Sandwichelementen der damit verbundene Wert $\mathbf{c}_{91}$ auf der sicheren Seite liegend mit dem statistisch ermittelten Abminderungsfaktor $\xi_{1}=0,9$ korrigiert. Aufgrund der untergeordneten Bedeutung des Kur- 
venverlaufs nach Überschreiten des Kontaktmomentes ist eine Abminderung des Werts $\mathrm{C}_{\vartheta 2}$ mit dem Abminderungsfaktor $\xi_{2}=0,5$ ausreichend.

\subsubsection{Berücksichtigung der Obergurtbreite und Anordnung der Verbindungs- mittel}

Die in den Abschnitten 3.3.2 und 3.3.3 aufgeführten Gleichungen ergeben sich aus Untersuchungen, die mit einem IPE-Profil 160 und alternierender Anordnung der Verbindungsmittel durchgeführt wurden. Entsprechend den Ergebnissen aus Abschnitt 3.2.5.8 besteht ein linearer Zusammenhang zwischen der Profilobergurtbreite $\mathrm{b}_{\mathrm{OG}}$ doppelsymmetrischer I-Träger und den Werten $\mathbf{c}_{91}$ und $\mathbf{c}_{92}$. Dieser Sachverhalt kann durch Umrechnung der Werte $c_{91}$ und $c_{92}$ mit Gleichung (3.12) berücksichtigt werden.

$$
c_{9 i}=c_{9 i(I P E 160)} \cdot\left(\frac{b_{O G}}{82 \mathrm{~mm}}\right) \quad \text { für Obergurtbreiten } \mathrm{b}_{\mathrm{OG}} \in[60 ; 100] \text { in }[\mathrm{mm}]
$$

Eine einseitige Anordnung der Verbindungsmittel bewirkt bei günstiger Drehrichtung der Pfette entsprechend Abschnitt 3.2.5.9 deutlich größere Werte $c_{92}$, während bei ungünstiger Drehrichtung die Werte $c_{\vartheta 2}$ gegen Null tendieren. Dieser Effekt darf durch die Erhöhung der Werte $\mathrm{C}_{92}$ nach Abschnitt 3.3.3 mit dem Faktor $\psi=1,5$ berücksichtigt werden, wenn bei einseitiger Anordnung der Verbindungsmittel sichergestellt ist, dass die Pfettenverdrehung nur in günstige Richtung erfolgen kann. Ist eine Pfettenverdrehung in ungünstiger Richtung nicht auszuschließen, ist auf der sicheren Seite liegend der Faktor $\psi=0$ (und damit $\mathbf{c}_{\vartheta 2}=0$ ) anzusetzen.

Bei Sandwichelementen, die nur mit einem Verbindungsmittel pro Element mit der Unterkonstruktion verbunden sind (beispielsweise im Fall einer verdeckten Befestigung), ist ebenfalls der Faktor $\psi=0$ anzusetzen, da für diese Art von Befestigung keine Ergebnisse experimenteller Untersuchungen vorliegen.

In allen Fällen bleibt der Wert $c_{91}$ durch die Anzahl und Anordnung der Verbindungsmittel unbeeinflusst und kann entsprechend den Abschnitten 3.3.2 und 3.3.3 aufgeführten Gleichungen berechnet werden. 


\subsection{Erstellung eines Rechenmodells}

Auf Grundlage des mechanischen Modells aus Abschnitt 3.1.7 und der in Tabelle 3.16 dargestellten Formeln zur Ermittlung der Werte $c_{91}$ und $c_{92}$ sowie des Kontaktmomentes $m_{K}$ kann für jedes System unterschiedlicher Profiltypen in Kombination mit Sandwich-Dach- und Wandelementen eine trilineare MomentenVerdrehungsbeziehung entsprechend Bild 3.56 ermittelt werden. Bei der Berechnung der Werte $\mathrm{c}_{91}$ und $\mathrm{C}_{\vartheta 2}$ wird die Befestigungsart durch den Faktor $\psi$ und bei Sandwich-Dachelementen mit Kernschicht aus Polyurethan-Hartschaum der Kriecheinfluss durch die Faktoren $\xi_{\mathrm{i}}$ berücksichtigt. Der Übergangsbereich von $\mathrm{C}_{91} \mathrm{zu}_{\mathrm{C}_{92}}$ im Bereich des Kontaktmomentes wird durch eine Gerade, die sich über den Bereich $2 \Delta \vartheta$ erstreckt, linearisiert, wobei sich der Wert $\Delta \vartheta$ als Differenz der Abszissenwerte $\vartheta$ zwischen den Ordinatenwerten $2 / 3 m_{k}$ und $m_{k}$ ergibt. Die maximal zulässige Verdrehung $\vartheta_{\text {grenz }}$ wird aufgrund der Ergebnisse aus Abschnitt 3.1 .5 auf 0,08 rad beschränkt.

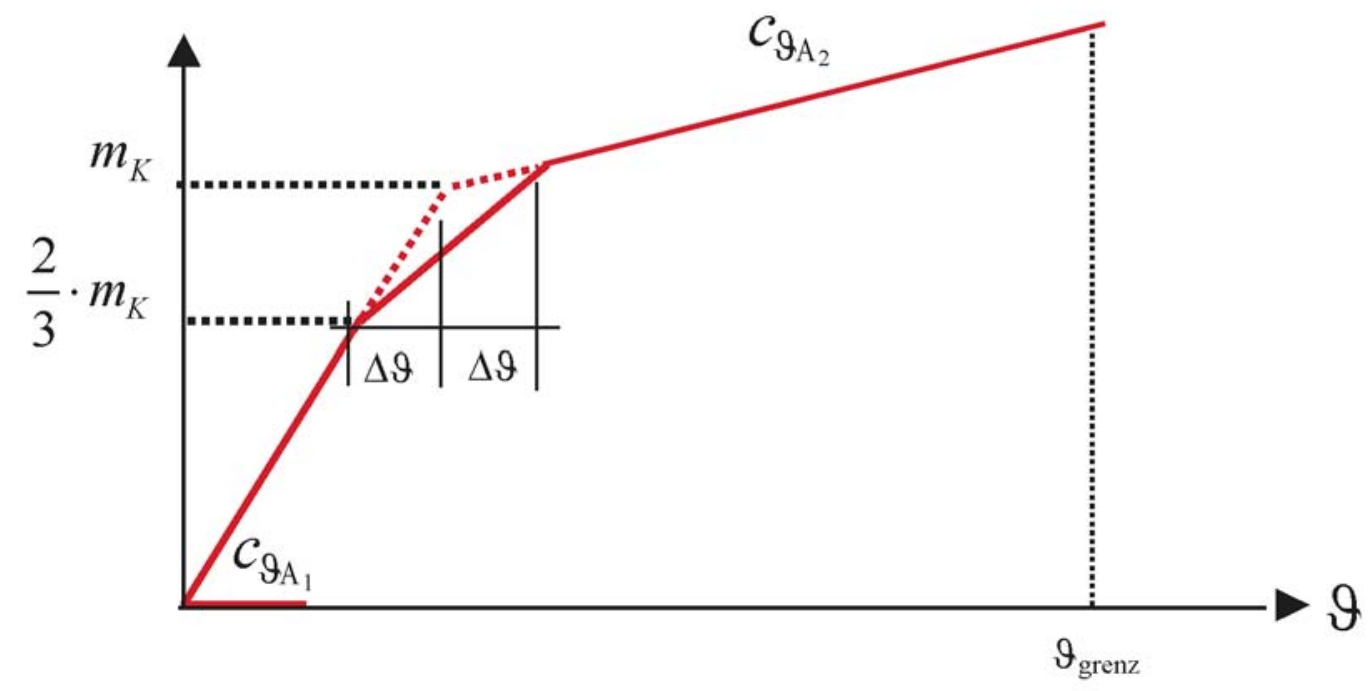

Bild 3.56: trilineare Idealisierung der Momenten-Verdrehungsbeziehung

Die Anschlusssteifigkeit der Sandwichelemente ergibt sich in Abhängigkeit der wirkenden Pfettenauflast aus der ermittelten Momenten-Verdrehungsbeziehung als Sekantensteifigkeit $\mathrm{C}_{\Im \mathrm{A}, \mathrm{k}}$ entsprechend Gleichung (3.13)

$$
c_{\vartheta A, k}=\frac{m_{K}}{\vartheta\left(m_{K}\right)}
$$

Mit der nach diesem Verfahren bestimmten Anschlusssteifigkeit $c_{\vartheta A, k}$ lässt sich entsprechend DIN 18800 Teil 2 die für den Nachweis der Biegedrillknicksicherheit benötigte Drehfedersteifigkeit $c_{\vartheta, k}$ bestimmen. 


\begin{tabular}{|c|c|c|c|}
\hline Systemkombination & $\mathrm{C}_{91}$ & $\mathrm{C}_{92}$ & Kontaktmoment $\mathrm{m}_{\mathrm{K}}$ \\
\hline Profil/Sandwichelement & {$[\mathrm{kNm} / \mathrm{m}]$} & {$[\mathrm{kNm} / \mathrm{m}]$} & {$[\mathrm{kNm} / \mathrm{m}]$} \\
\hline $\begin{array}{c}\text { doppelsymmetrischer I-Träger } \\
\text { PUR-Dachelement }\end{array}$ & $\xi_{1} \cdot 1,60 \cdot E_{s} \cdot \frac{b_{O G}}{82 m m}$ & $\xi_{2} \cdot \psi \cdot 0,44 \cdot t_{K} \cdot E_{s} \cdot \frac{b_{O G}}{82 m m}$ & $q \cdot \frac{b_{O G}}{2}$ \\
\hline $\begin{array}{c}\text { doppelsymmetrischer I-Träger } \\
\text { PUR-Wandelement }\end{array}$ & $1,20 \cdot E_{s} \cdot \frac{b_{O G}}{82 m m}$ & $\psi \cdot 0,38 \cdot t_{K} \cdot E_{s} \cdot \frac{b_{O G}}{82 m m}$ & $q \cdot \frac{b_{O G}}{2}$ \\
\hline $\begin{array}{c}\text { doppelsymmetrischer I-Träger } \\
\text { Mineralfaser-Dachelement }\end{array}$ & $0,69 \cdot E_{s} \cdot \frac{b_{O G}}{82 m m}$ & $\psi \cdot 0,18 \cdot t_{K} \cdot E_{s} \cdot \frac{b_{O G}}{82 m m}$ & $q \cdot \frac{b_{O G}}{2}$ \\
\hline $\begin{array}{c}\text { doppelsymmetrischer I-Träger } \\
\text { Mineralfaser-Wandelement }\end{array}$ & $0,48 \cdot E_{s} \cdot \frac{b_{O G}}{82 m m}$ & $\psi \cdot 0,16 \cdot t_{K} \cdot E_{s} \cdot \frac{b_{O G}}{82 \mathrm{~mm}}$ & $q \cdot \frac{b_{O G}}{2}$ \\
\hline $\begin{array}{c}\text { Z-Profil } \\
\text { PUR-Dachelement } \\
\text { (günstige Drehrichtung) }\end{array}$ & $\xi_{1} \cdot 1,60 \cdot E_{s}$ & 0 & $q \cdot b_{Z}$ \\
\hline $\begin{array}{c}\text { Z-Profil } \\
\text { PUR-Wandelement } \\
\text { (günstige Drehrichtung) }\end{array}$ & $1,20 \cdot E_{s}$ & 0 & $q \cdot b_{Z}$ \\
\hline $\begin{array}{c}\text { Z-Profil } \\
\text { Mineralfaser-Dachelement } \\
\text { (günstige Drehrichtung) }\end{array}$ & $0,69 \cdot E_{s}$ & 0 & $q \cdot b_{Z}$ \\
\hline $\begin{array}{c}\text { Z-Profil } \\
\text { Mineralfaser-Wandelement } \\
\text { (günstige Drehrichtung) }\end{array}$ & $0,48 \cdot E_{s}$ & 0 & $q \cdot b_{Z}$ \\
\hline $\begin{array}{c}\text { Z-Profil } \\
\text { unabhängig vom Elementtyp } \\
\text { (ungünstige Drehrichtung) }\end{array}$ & 0 & 0 & 0 \\
\hline
\end{tabular}

$\xi \quad$ Faktor zur Berücksichtigung des Kriecheinflusses bei Dachelementen mit PUR-Schaumkern

$$
\begin{aligned}
& \xi_{1}=0,9 \\
& \xi_{2}=0,5
\end{aligned}
$$

$\psi \quad$ Faktor zur Berücksichtigung der Befestigungsart

$$
\begin{array}{ll}
\psi=1 & \text { für alternierende Befestigung } \\
\psi=1,5 & \text { für einseitige Befestigung (ungünstige Drehrichtung ausgeschlossen) } \\
\psi=0 & \text { für einseitige Befestigung (ungünstige Drehrichtung nicht ausgeschlossen) } \\
\psi=0 & \text { für verdeckte Befestigung }
\end{array}
$$

Die Formeln gelten für Sandwichelemente mit

- Kernschicht aus Polyurethan oder Mineralfasern mit $E_{s} \in[2,0 ; 6,0]$ in $\left[\mathrm{N} / \mathrm{mm}^{2}\right]$

und für

- Kernblechdicke der äußeren Deckschicht $\mathrm{t}_{\mathrm{K}} \in[0,42 ; 0,67]$ in [mm]

- doppelsymmetrische I-Träger mit Obergurtbreiten $b_{\mathrm{OG}} \in[60 ; 100]$ in [mm]

- Z-Profile mit Mindestblechdicke $t_{N}=2,0 \mathrm{~mm}$ und Flanschbreite $b_{Z} \geq 60 \mathrm{~mm}$

Tabelle 3.16: Zusammenstellung der Formeln und Anwendungsgrenzen 


\section{Untersuchungen zur Stabilisierung durch Schubsteifigkeit}

\subsection{Experimentelle Schubfelduntersuchungen}

\subsubsection{Versuchsaufbau}

Zur Ermittlung der Schubsteifigkeit zweiseitig gelagerter Scheiben aus Trapezprofilen wurden experimentelle Schubfelduntersuchungen auf dem horizontalen Schubrahmen der Versuchsanstalt für Stahl, Holz und Steine der Universität Karlsruhe (TH) sowohl mit zweiseitiger als auch mit vierseitiger Lagerung durchgeführt.

Der Schubrahmen ist entsprechend Bild 4.1 als Vier-Gelenk-Rahmen ausgebildet.

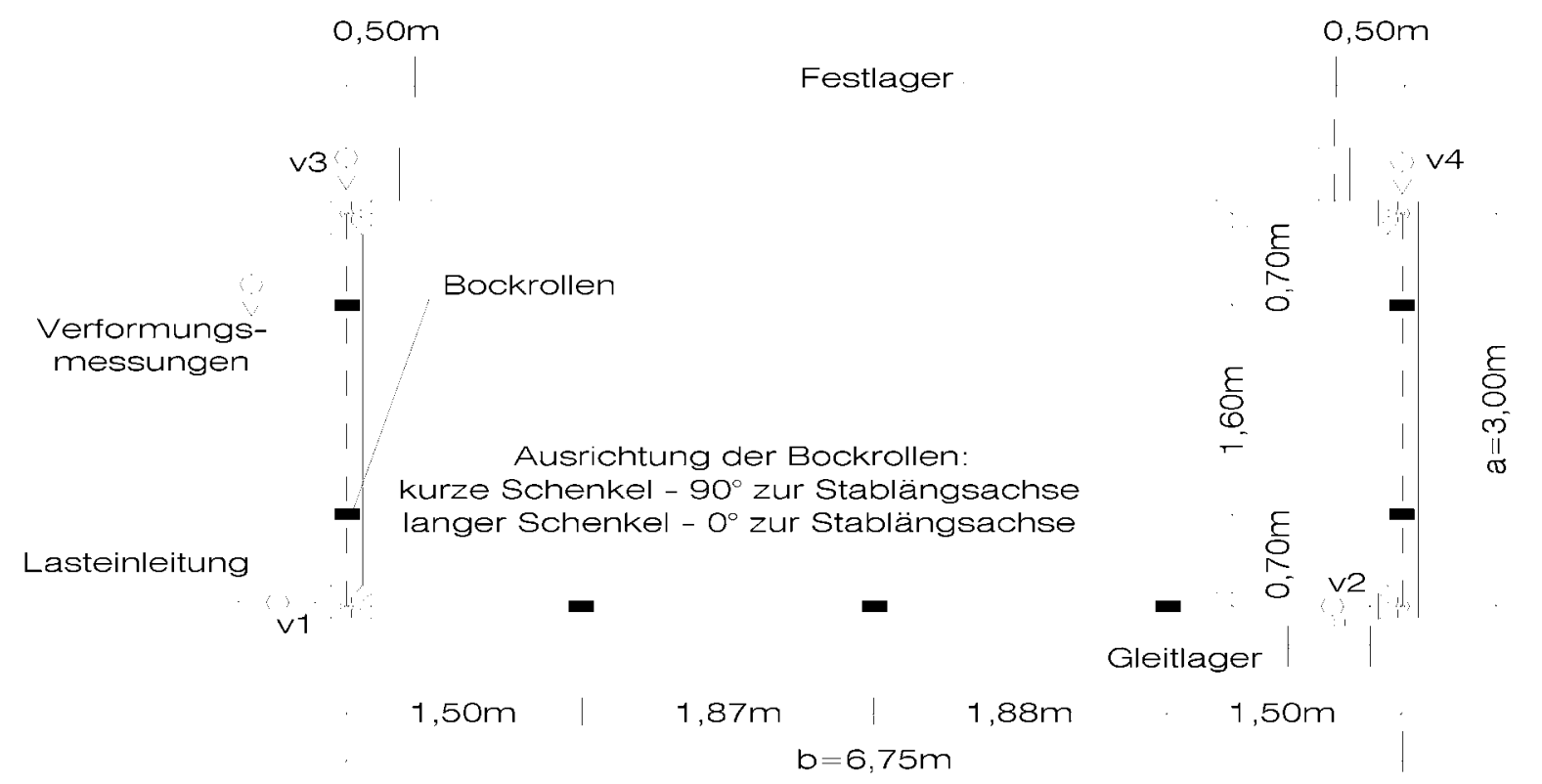

Bild 4.1: Grundrisszeichnung des Schubrahmens

Das Achsmaß a des Rahmens beträgt 3,0 m und entspricht der Schubfeldlänge, das Achsmaß b beträgt $6,75 \mathrm{~m}$ und entspricht der Schubfeldbreite. Alle vier Rahmenschenkel bestehen aus Profilen HE 200 B nach DIN 1025-2 mit zusätzlichen Profilen U 200 nach DIN 1026, die liegend auf dem Obergurt der Profile HE 200 B als Verschleißprofil montiert sind (siehe Bild 4.2).

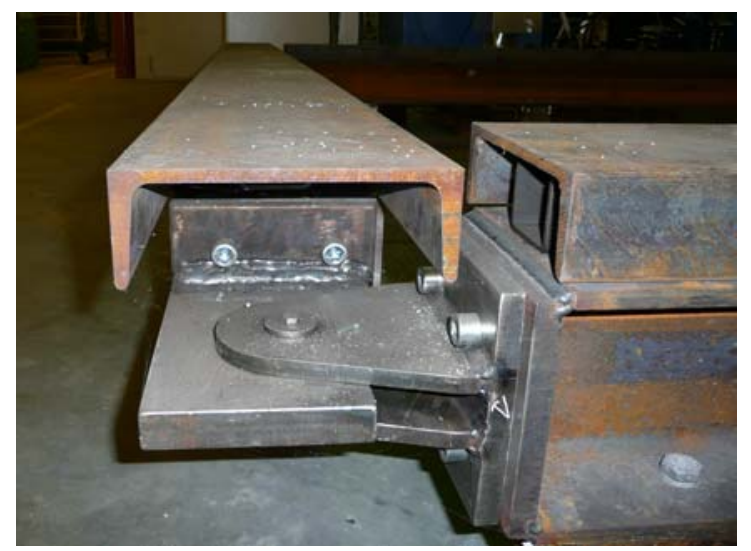

Bild 4.2: Ausbildung der Eckgelenke des Schubrahmens und Aufbau der Rahmenschenkel 
Einer der beiden langen Schenkel des Rahmens ist mittels zweier Festlager starr gelagert. Die übrigen drei Schenkel sind durch ihre Lagerung auf Bockrollen in der Ebene verschieblich. Bild 4.6 zeigt den Schubrahmen ohne Eindeckung in unausgelenkter Stellung.

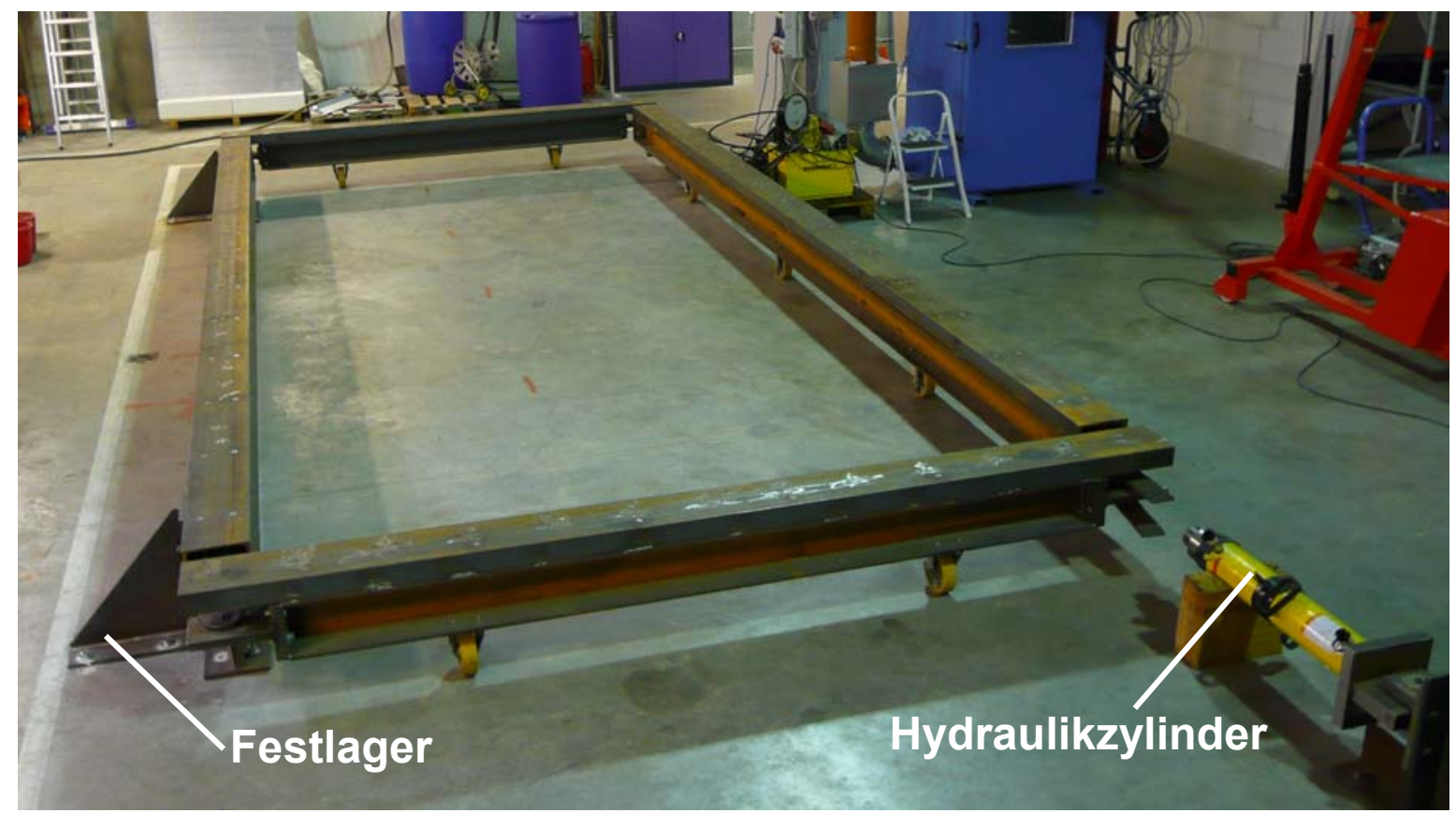

Bild 4.3: Schubrahmen ohne Eindeckung in unausgelenkter Stellung

Die Belastung des Rahmens erfolgt am frei beweglichen langen Schenkel durch einen 500 kN-Hydraulikzylinder des Typs HW RR 5020, Fabrikat Enerpac, der entweder durch eine elektrische Hochleistungspumpe oder durch eine hydraulische Handpumpe mit jeweils 700 bar maximalem Arbeitsdruck gespeist wird. Durch den Zylinderhub nimmt der ursprünglich rechtwinklig ausgerichtete Rahmen die Form eines Parallelogramms an. Um ein Ausweichen aus der Ebene zu verhindern, wurde die frei bewegliche Rahmenecke entsprechend Bild 4.7 durch ein im Boden verankertes Gleitlager gegen Abheben gesichert.

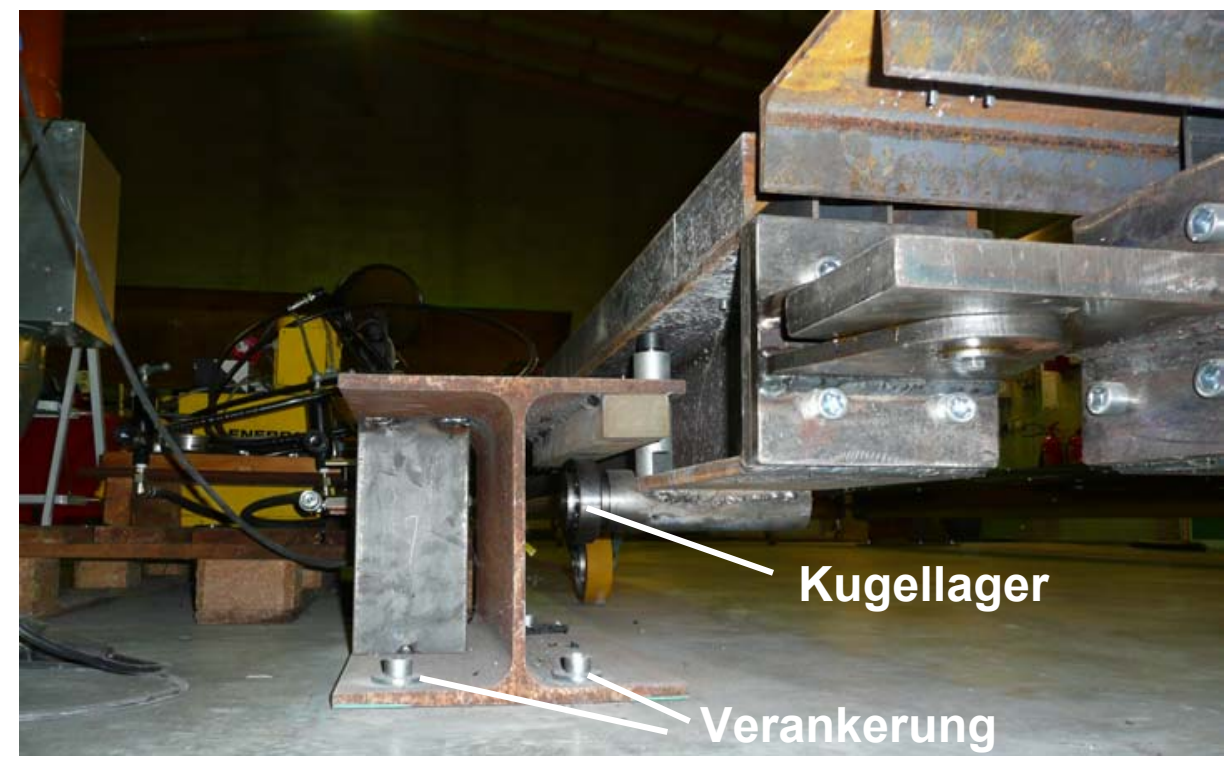

Bild 4.4: Ausbildung des Gleitlagers 
Mit einem 500 kN-Präzisions-Kraftaufnehmer des Typs U10M, Fabrikat HBM, wird die Belastung des Rahmens kontinuierlich gemessen. Der Bereich der Lasteinleitung in die Rahmenkonstruktion ist im Bild 4.5 ersichtlich.

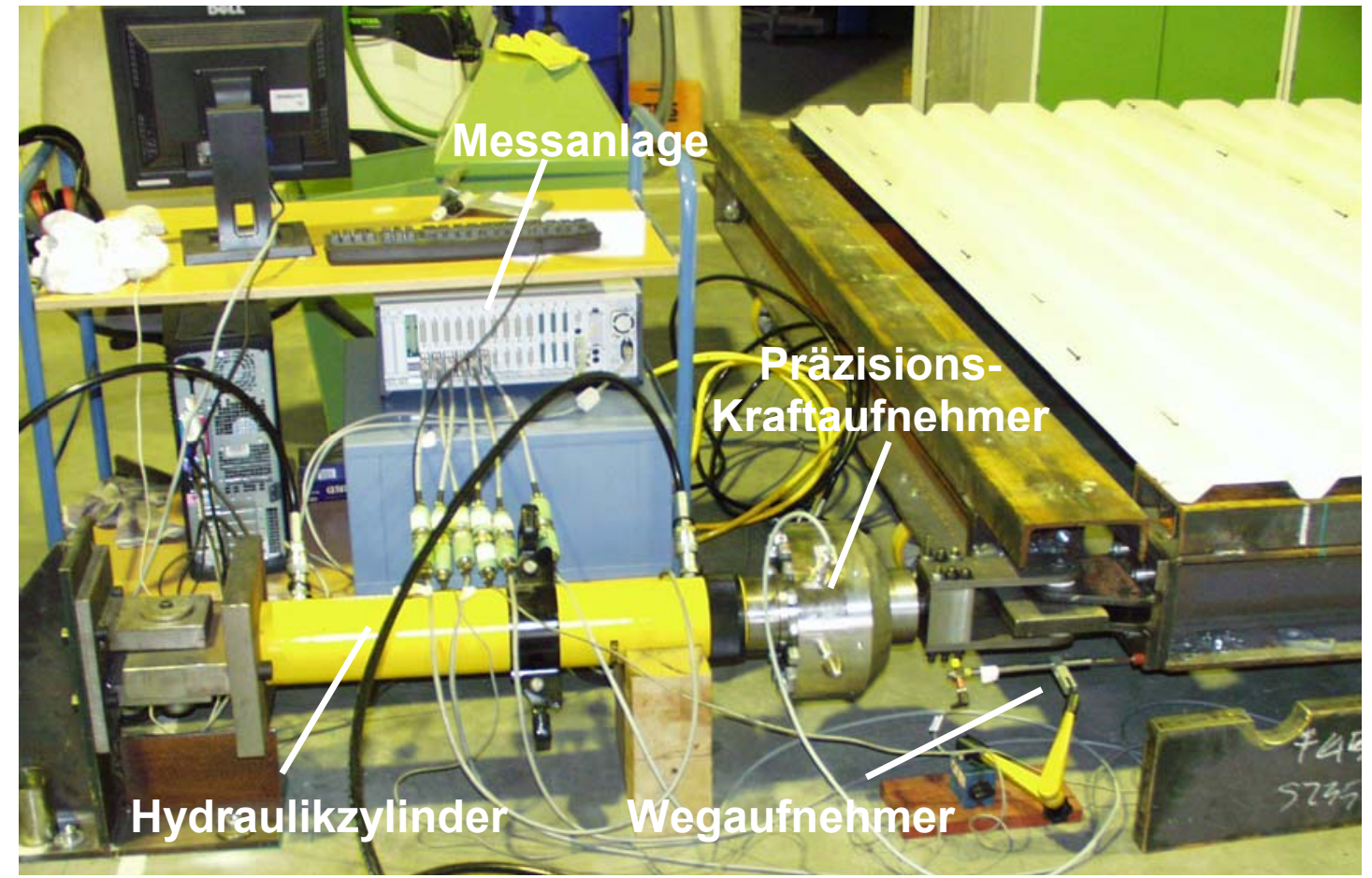

Bild 4.5: Lasteinleitungsbereich

Die Rahmenverschiebungen $v_{1}$ und $v_{2}$ infolge der aufgebrachten Belastung werden entlang des frei beweglichen langen Schenkels mit induktiven Wegaufnehmern gemessen. Ebenso werden zur Bestimmung der Rahmennachgiebigkeiten an den Rahmenecken in unmittelbarer Nähe der Festlager die Verschiebungen $v_{3}$ und $v_{4}$ gemessen. Damit ergibt sich die effektive Schubfeldverformung $v_{s} z u$

$$
v_{S}=\frac{v_{1}+v_{2}}{2}-\frac{a}{b}\left(v_{3}-v_{4}\right)
$$

Die Aufzeichnung aller Daten (Kräfte und Verschiebungen) erfolgte kontinuierlich mit einer Datenerfassungsrate von 2 Hertz mittels einer elektronischen Messanlage.

Die Versuchsdurchführung ohne Trapezbleche ergab, dass der Widerstand der Versuchseinrichtung (Reibung in den Eckgelenken, Reibung der Bockrollen, etc.) im Vergleich zu dem der Trapezbleche so gering war, dass er für die weiteren Untersuchungen vernachlässigt werden konnte.

\subsubsection{Versuchsgegenstand}

Die Versuche wurden mit Trapezprofilen LS 5/35/1035 der Firma Arcelor Construction France (siehe Bild 4.6) durchgeführt, die eine Nennblechdicke $t_{N}=1,0 \mathrm{~mm}$ hatten und einer Charge der Stahlsorte S350GD+Z entstammten. Dieses Trapezprofil ist weit verbreitet und befindet sich im Standardprogramm fast aller Profilhersteller. 


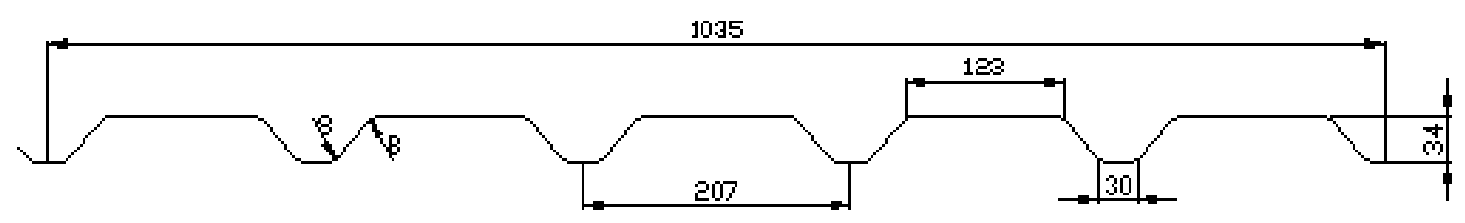

Bild 4.6: Nennabmessungen der Querschnittsgeometrie des Trapezprofils LS 5/35/1035

Zur Berücksichtigung des Einflusses einer von den Nennabmessungen des Profils abweichenden Querschnittsgeometrie wurde vor der Versuchsdurchführung die Geometrie der Profiltafeln bestimmt und protokolliert. Die ermittelten Querschnittswerte sind der Tabelle $4.1 \mathrm{zu}$ entnehmen.

\begin{tabular}{|c|c|c|c|c|c|}
\hline Kennwert & 1. Rippe & 2. Rippe & 3. Rippe & 4. Rippe & 5. Rippe \\
\hline Profilhöhe & 33,5 & 33,5 & 34,0 & 33,2 & 33,0 \\
\hline Obergurtbreite & 124,0 & 123,0 & 123,0 & 124,0 & 124,0 \\
\hline Untergurtbreite & 30,5 & 30,0 & 29,5 & 29,5 & 30,5 \\
\hline Einlaufbreite & 1035 & \multicolumn{5}{l}{} \\
\cline { 1 - 2 } Auslaufbreite & 1033 &
\end{tabular}

Tabelle 4.1: Abmessungen des Profils LS 5/35/1035; Istwerte in [mm]

Entsprechend Abschnitt 3.1.6.2 wurden die Kernblechdicken sowie die mechanischen Eigenschaften der Trapezprofile nach DIN EN 10002-1 bestimmt. Die ermittelten Werte sind in Tabelle 4.2 aufgeführt.

\begin{tabular}{|c|c|c|c|c|c|}
\hline Probennummer & Nennblechdicke $t_{\mathrm{N}}$ & Kernblechdicke $t_{\mathrm{K}}$ & $\mathrm{R}_{\mathrm{eH}} / \mathrm{R}_{\mathrm{p} 0,2}$ & $\mathrm{R}_{\mathrm{m}}$ & $\mathrm{A}_{80 \mathrm{~mm}}$ \\
\hline & {$[\mathrm{mm}]$} & {$[\mathrm{mm}]$} & {$\left[\mathrm{N} / \mathrm{mm}^{2}\right]$} & {$\left[\mathrm{N} / \mathrm{mm}^{2}\right]$} & {$[\%]$} \\
\hline LS 5/35/1035_a & 1,00 & 0,95 & 423 & 439 & 21 \\
\hline LS 5/35/1035_b & 1,00 & 0,96 & 424 & 438 & 21 \\
\hline LS 5/35/1035_c & 1,00 & 0,97 & 425 & 439 & 23 \\
\hline
\end{tabular}

Tabelle 4.2: Blechdicken und mechanische Eigenschaften der Trapezprofile; Istwerte

Die Profiltafeln wurden mit ihrer Längsrichtung parallel zu den kurzen Schenkeln des Rahmens verlegt, so dass die Lasteinleitung rechtwinklig zur Profilierung erfolgte. Die Befestigung auf dem Schubrahmen erfolgte am Querrand mit Bohrschrauben des Typs EJOT JT2-12-5,5x35 V16 [14] in jedem Untergurt des Trapezprofils. Bei der vierseitigen Lagerung wurden die Profiltafeln zudem mit Bohrschrauben des gleichen Typs entlang der Längsränder im Abstand $e_{R}=150 \mathrm{~mm}$ verschraubt. Die Verbindung der Profiltafeln miteinander erfolgte stets mit Bohrschrauben des Typs EJOT JT2-4,8x19 [14], die im Längsstoß im Abstand $e_{L}=150 \mathrm{~mm}$ angeordnet waren.

Eine Gesamtansicht des eingedeckten Schubrahmens ist Bild 4.7 für vierseitige Lagerung und Bild 4.8 für zweiseitige Lagerung der Trapezprofile LS 5/35/1035 zu entnehmen. 


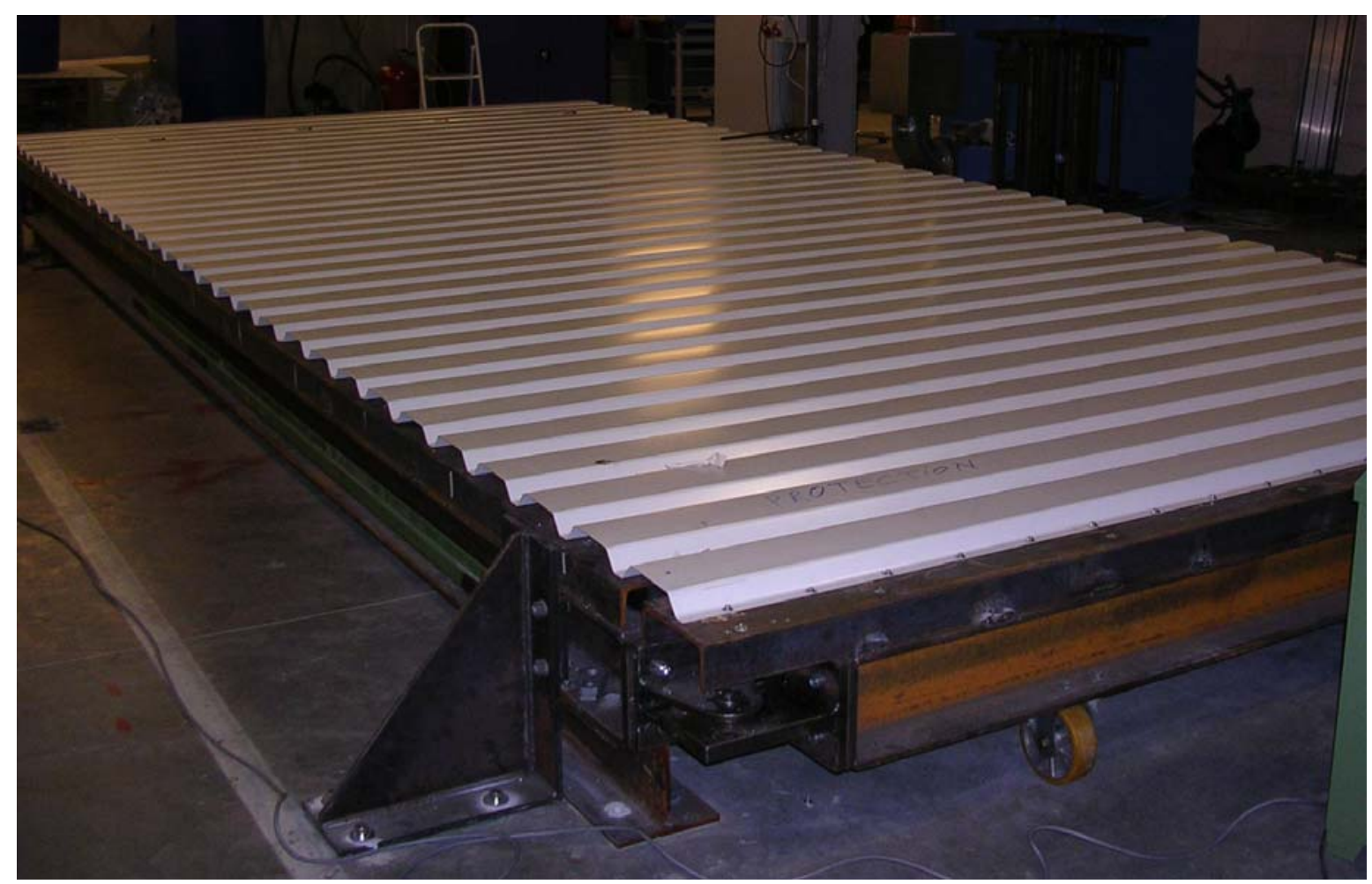

Bild 4.7: eingedeckter Schubrahmen mit vierseitig gelagertem Trapezblech

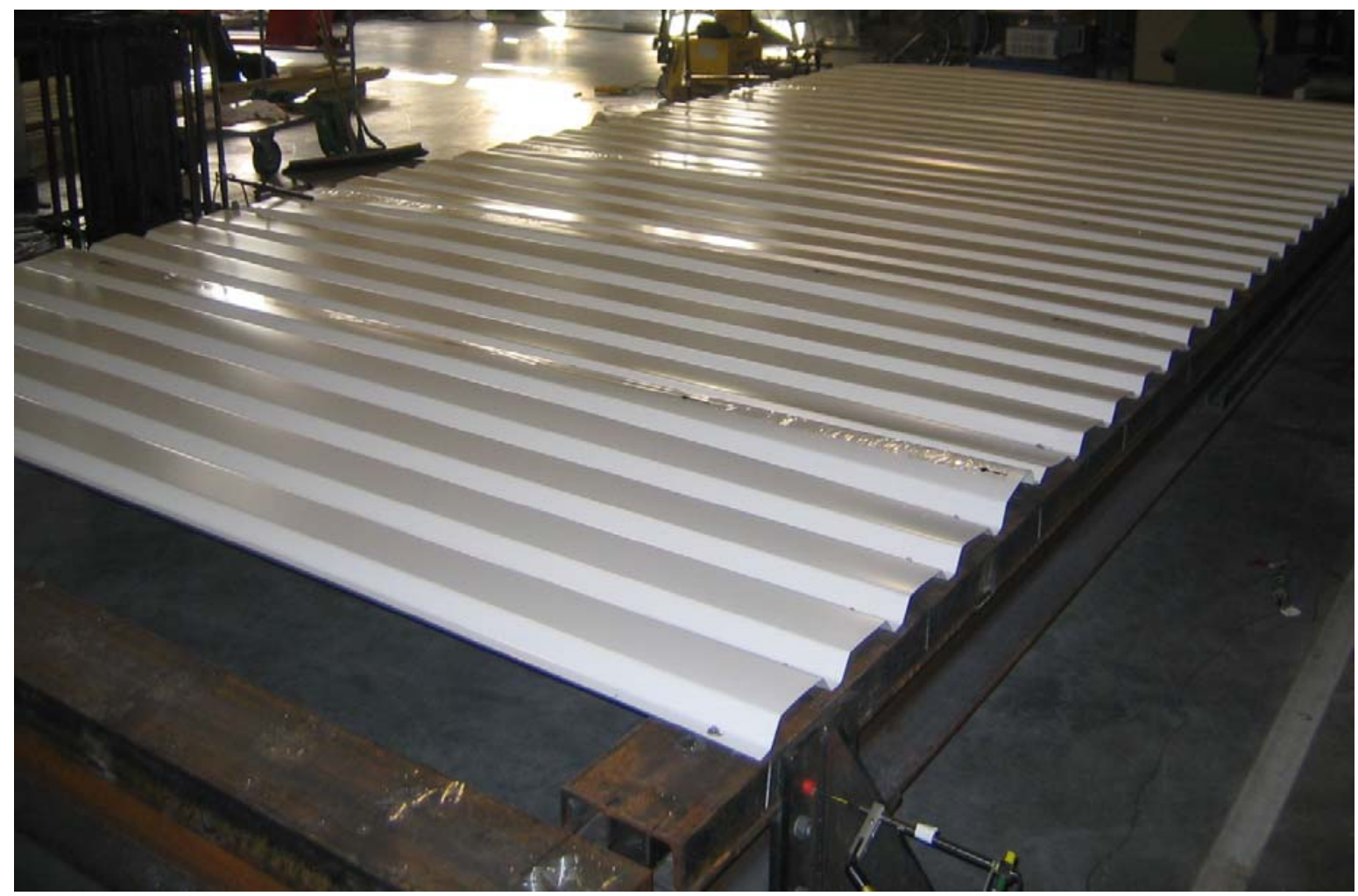

Bild 4.8: eingedeckter Schubrahmen mit zweiseitig gelagertem Trapezblech 


\subsubsection{Versuchsdurchführung und Ergebnisse}

Das System wird vom unausgelenkten und kraftfreien Zustand des Schubrahmens aus durch zyklische Belastungsstufen (vgl. Bild 4.11) mit einer Vorschubgeschwindigkeit des Kolbens von $0,1 \mathrm{~mm} / \mathrm{s}$ beansprucht. Nach jeder Laststufe erfolgt eine Entlastung, um bleibende Verformungen und Steifigkeitsänderungen beobachten zu können. Die Versuchsbeschreibung und Betrachtung zum Lastverformungsverhalten sowie eine Beschreibung des Versagensverhaltens sind in Tabelle 4.3 aufgeführt.

\begin{tabular}{|c|c|c|c|c|}
\hline & \multicolumn{2}{|r|}{ Versuch 1} & \multicolumn{2}{|c|}{ Versuch 2} \\
\hline Lagerungsart & & vierseitig & \multicolumn{2}{|c|}{ zweiseitig } \\
\hline $\begin{array}{l}\text { Schubfeldlänge a } \\
\text { Schubfeldbreite b }\end{array}$ & & $\begin{array}{l}3000 \mathrm{~mm} \\
6831 \mathrm{~mm}\end{array}$ & \multicolumn{2}{|c|}{$\begin{array}{l}3000 \mathrm{~mm} \\
6624 \mathrm{~mm}\end{array}$} \\
\hline $\begin{array}{l}\text { Beobachtungen } \\
\text { während der } \\
\text { Versuchsdurch- } \\
\text { führung }\end{array}$ & $\begin{array}{l}100 \mathrm{kN} \\
(\mathrm{T}=14,6 \mathrm{kN} / \mathrm{m})\end{array}$ & $\begin{array}{l}\text { erste sichtbare bleiben- } \\
\text { de Verformungen } \\
\text { deutlich sichtbare Blech- } \\
\text { endverwölbung der } \\
\text { Profilrippen am Quer- } \\
\text { rand (siehe Bild 4.9) } \\
\\
\text { Versagen der ersten } \\
\text { Verbindungsmittel am } \\
\text { Querrand durch Absche- } \\
\text { ren }\end{array}$ & $\begin{array}{l}105 \mathrm{kN} \\
(\mathrm{T}=15,9 \mathrm{kN} / \mathrm{m}) \\
121 \mathrm{kN} \\
(\mathrm{T}=18,3 \mathrm{kN} / \mathrm{m})\end{array}$ & $\begin{array}{l}\text { erste sichtbare bleiben- } \\
\text { de Verformungen } \\
\text { deutlich sichtbare } \\
\text { Blechendverwölbung } \\
\text { der Profilrippen am } \\
\text { Querrand } \\
\text { Knicke in der druckbe- } \\
\text { anspruchten Randrippe } \\
\text { (siehe Bild 4.10) } \\
\text { Versagen der ersten } \\
\text { Verbindungsmittel am } \\
\text { Querrand durch Ab- } \\
\text { scheren }\end{array}$ \\
\hline
\end{tabular}

Tabelle 4.3: Versuchsbeschreibung und Ergebnisse

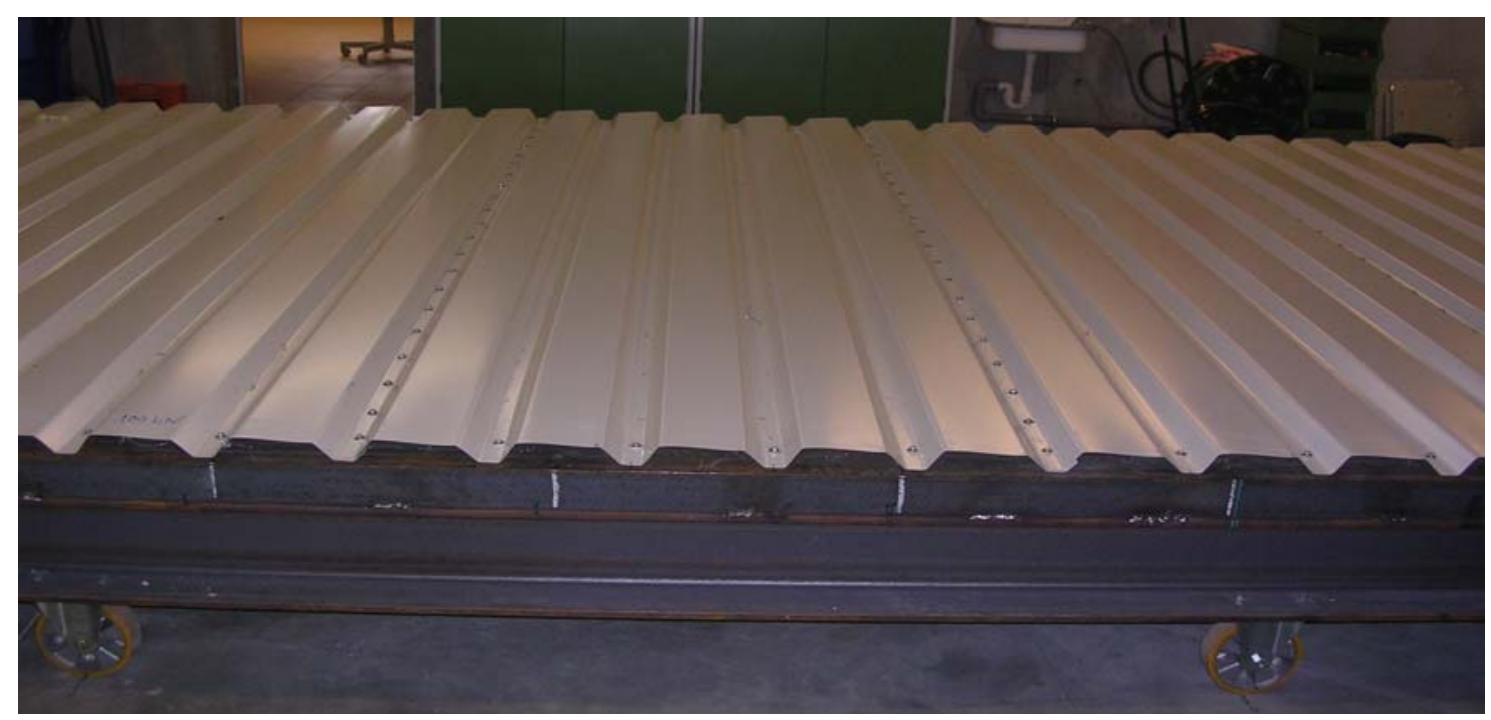

Bild 4.9: Blechendverwölbung beim vierseitig gelagerten Trapezprofil (Belastung $100 \mathrm{kN}, T=14,6 \mathrm{kN} / \mathrm{m}$ ) 


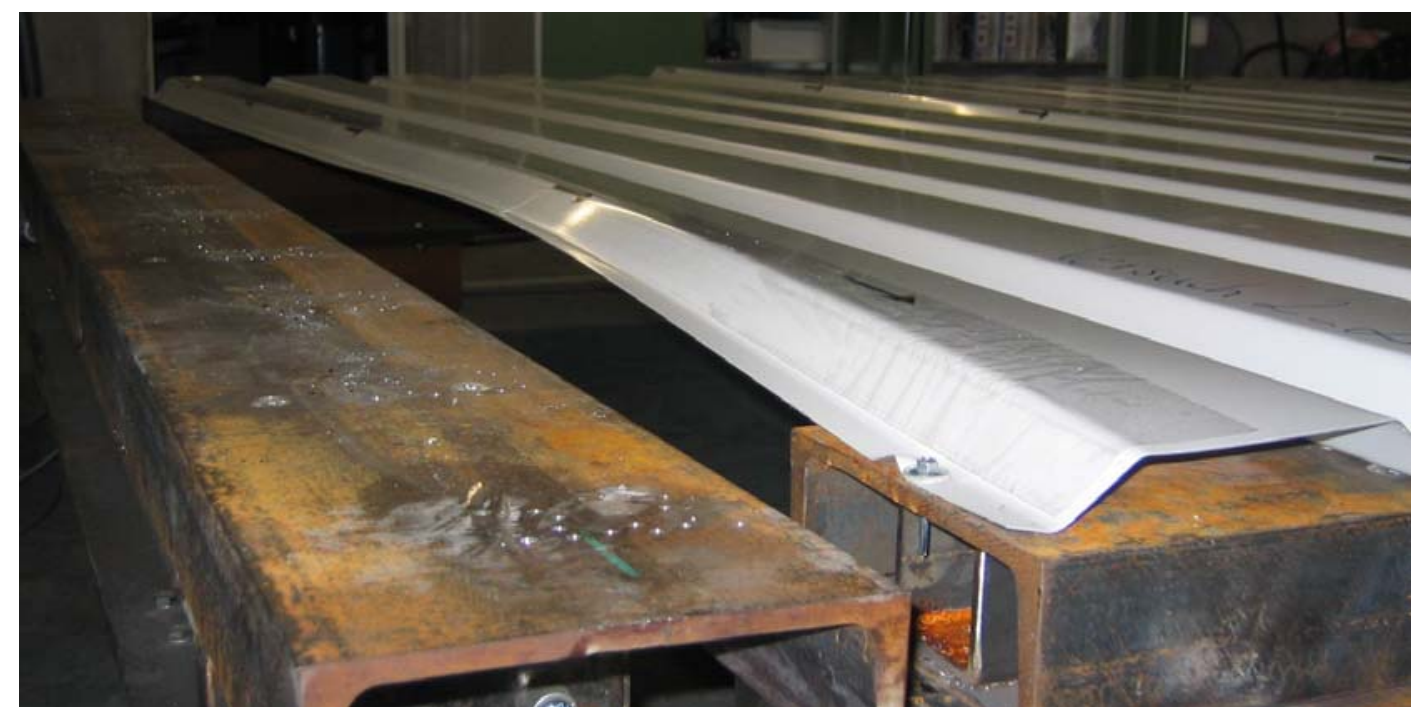

Bild 4.10: Deformationen in der druckbeanspruchten Randrippe bei etwa $105 \mathrm{kN}(T=15,9 \mathrm{kN} / \mathrm{m})$

Bei beiden Versuchen trat das Versagen durch Abscheren der Verbindungsmittel am Querrand auf. Die Stelle, an der die Verbindungsmittel zuerst versagten, befand sich jeweils an einem der nächstgelegenen Längsstöße zum Eckbereich. Nach der Demontage der Profiltafeln wurden im Fall der zweiseitig gelagerten Trapezblechscheibe festgestellt, dass sich insbesondere im Eckbereich erhebliche Langlöcher gebildet hatten. Bei der allseitig gelagerten Trapezblechscheibe waren die Langlöcher deutlich weniger ausgeprägt.

Die Darstellung der aufgezeichneten Versuchsdaten erfolgt als Schubfluss T über der resultierenden Schubfeldverformung $v_{s}$. Das für beide Lagerungsarten ermittelte Last-Verformungsdiagramm ist dem Bild 4.11 zu entnehmen.

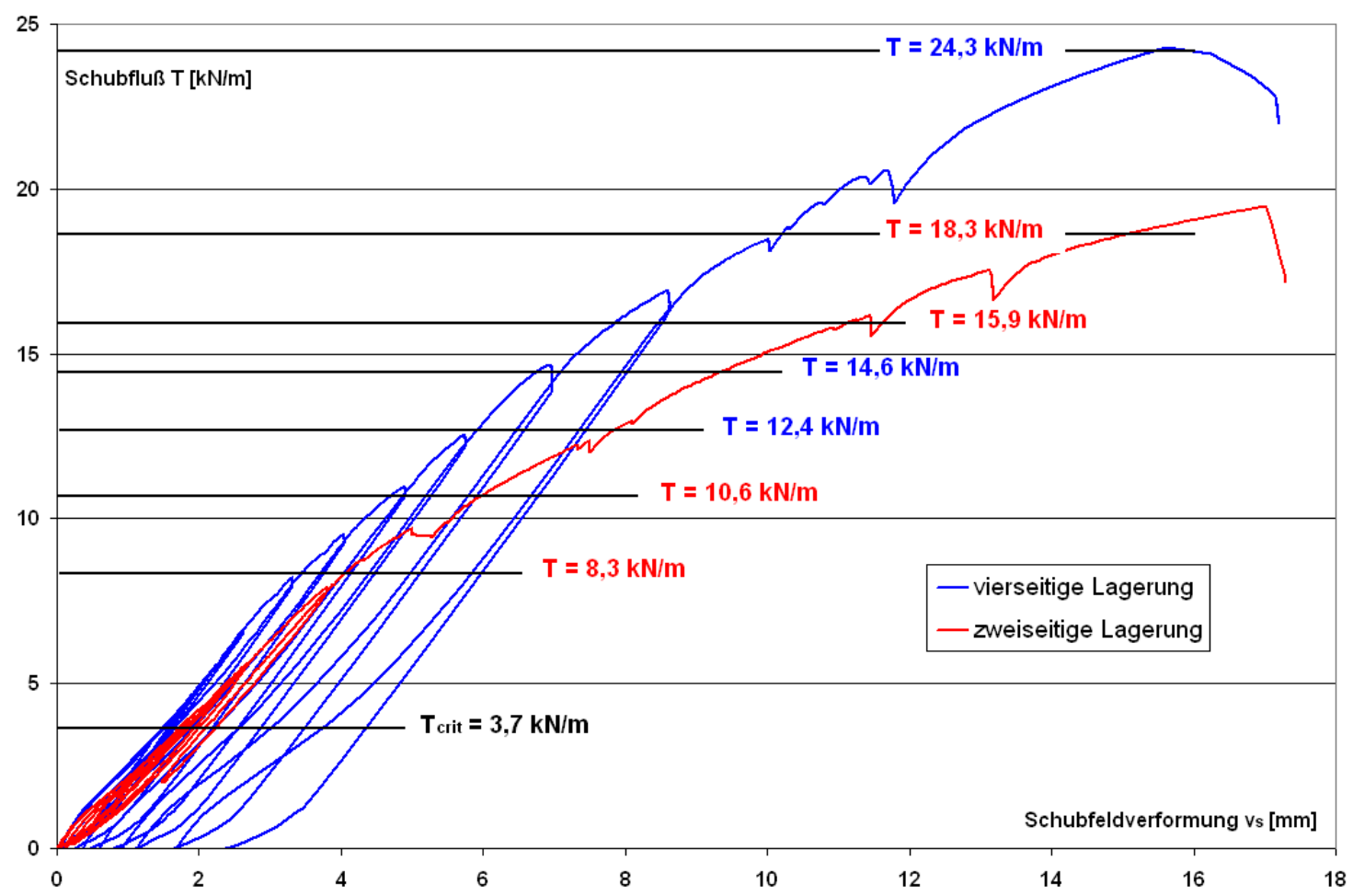

Bild 4.11: Last-Verformungsdiagramm aus Schubfeldversuchen mit zwei- und vierseitiger Lagerung 


\subsubsection{Versuchsauswertung}

Die Schubsteifigkeit S eines ausgelenkten Schubfeldes gemäß Bild 4.12 lässt sich allgemein nach Gleichung (4.2) berechnen.

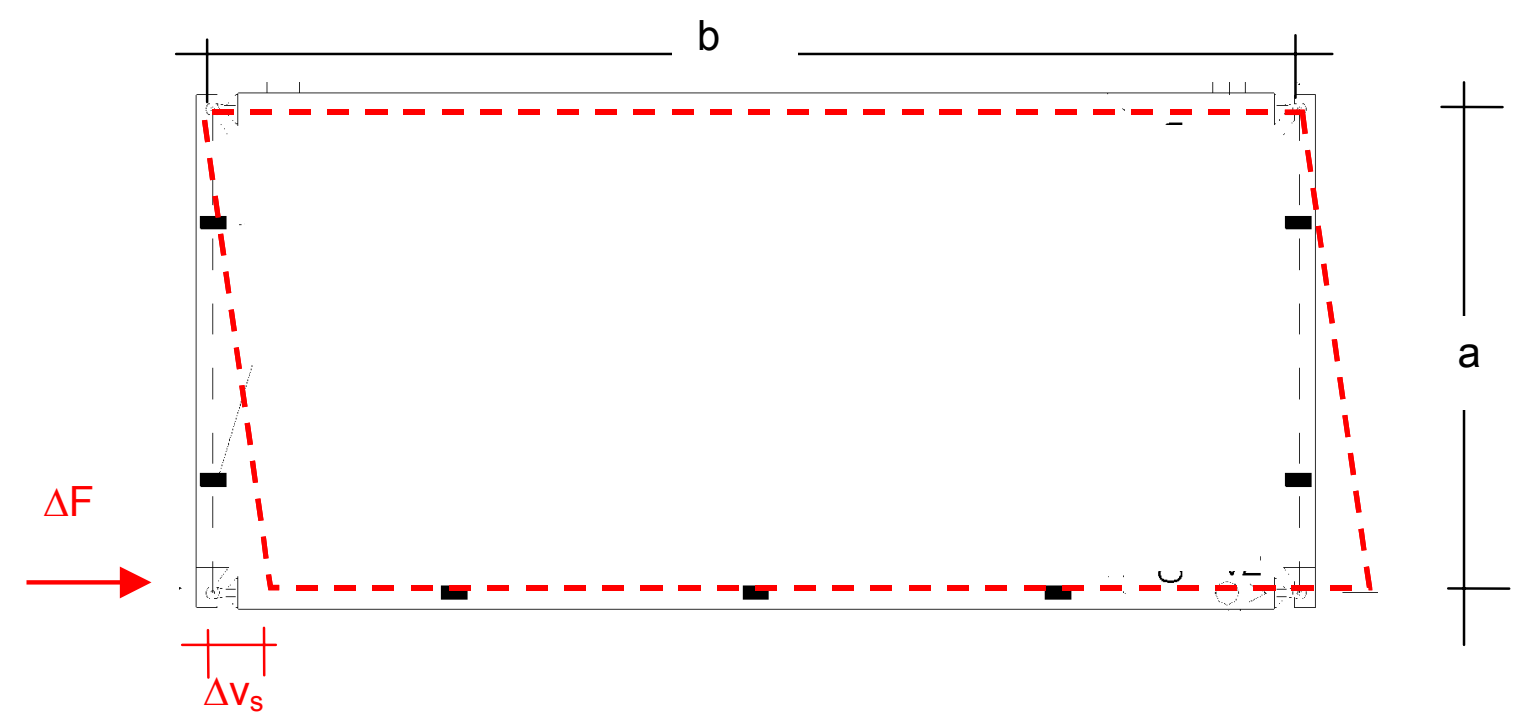

Bild 4.12: ausgelenktes Schubfeld

$$
S=\frac{\Delta T}{\Delta \gamma}=\frac{\Delta F \cdot a}{b \cdot \Delta v_{S}}
$$

mit

$$
\begin{aligned}
& \Delta \mathrm{F}=\text { Lastinkrement } \\
& \Delta \mathrm{v}_{\mathrm{S}}=\text { Inkrement der Gesamtschubfeldverformung } \\
& \mathrm{a}=\text { Schubfeldlänge } \\
& \mathrm{b}=\text { Schubfeldbreite }
\end{aligned}
$$

und

$$
\begin{aligned}
& \Delta T=\frac{\Delta F}{b} \quad \text { Inkrement des Schubflusses } \\
& \Delta \gamma=\frac{\Delta v_{S}}{a} \quad \text { zugehöriges Inkrement des Schubfeldwinkels }
\end{aligned}
$$

Die Schubsteifigkeit der zuvor beschriebenen Schubfeldversuche wird als Sekantensteifigkeit bei dem auf Grundlage der Elastizitätstheorie nach [52] ermittelten kritischen Schubfluss $T_{\text {crit }}$ bestimmt. Dieser ergibt sich entsprechend DIN 18807 Teil 1 als Minimum dreier Kriterien für die Begrenzung der Belastbarkeit von Schubfeldern für das untersuchte Trapezprofil mit Hilfe von [53] zu:

$$
T_{\text {crit }}=\min \left\{\begin{array}{c}
\text { zul } T_{1} \\
\text { zul } T_{2} \\
\text { zul } T_{3}
\end{array}\right\}=\min \left\{\begin{array}{c}
3,71 \mathrm{kN} / \mathrm{m} \\
6,77 \mathrm{kN} / \mathrm{m} \\
10,89 \mathrm{kN} / \mathrm{m}
\end{array}\right\}=3,71 \mathrm{kN} / \mathrm{m}
$$

Die nach diesem Verfahren ermittelte Schubsteifigkeit $S$ ist zusammen mit den Versagenslasten in Tabelle 4.4 aufgeführt. 


\begin{tabular}{|c|c|c|c|c|c|}
\hline $\begin{array}{c}\text { Versuchs- } \\
\text { Nr. }\end{array}$ & $\begin{array}{c}\text { Schubsteifigkeit S } \\
\text { bei } T_{\text {crit }}\end{array}$ & $\begin{array}{c}\text { Schubfeldverfor- } \\
\text { mung } \text { v }_{\mathbf{s}} \text { bei } T_{\text {crit }}\end{array}$ & $\begin{array}{c}\text { Versagenslast } \\
\text { (Schubtragfähigkeit) }\end{array}$ & $\begin{array}{c}\text { max. } \\
\text { Schubfluss T }\end{array}$ & $\begin{array}{c}\text { max. Schubfeld- } \\
\text { verformung v }\end{array}$ \\
\hline$[\mathrm{kN} / \mathrm{m}]$ & {$[\mathrm{mm}]$} & {$[\mathrm{kN}]$} & {$[\mathrm{kN} / \mathrm{m}]$} & {$[\mathrm{mm}]$} \\
\hline 1 & 7350 & 1,5 & 165,9 & 24,3 & 17,2 \\
\hline 2 & 6185 & 1,8 & 120,9 & 19,5 & 17,3 \\
\hline
\end{tabular}

Tabelle 4.4: Auswertung der experimentellen Schubfelduntersuchungen

Der Unterschied zwischen zweiseitiger und vierseitiger Lagerung beträgt demnach etwa $19 \%$ bezüglich der Schubsteifigkeit und etwa $25 \%$ bezüglich des maximalen Schubflusses.

\subsubsection{Nachgiebigkeit der Verbindungsmittel}

Die zur Befestigung der Trapezblechscheiben auf dem Schubrahmen eingesetzten Verbindungsmittel werden maßgeblich auf Abscheren beansprucht. Die aufgrund dieser Beanspruchung entstehenden Anschlussnachgiebigkeiten mindern die Schubfeldsteifigkeit. Da bei der zweiseitigen Lagerung die Verbindungsmittel am Querrand zusätzlich zur planmäßigen Beanspruchung auch Kräfte in Rippenlängsrichtung abtragen müssen und dadurch teilweise deutlich größere Beanspruchungen erfahren, darf die Nachgiebigkeit der Verbindungsmittel nicht vernachlässigt werden.

Für den Vergleich mit den experimentellen Ergebnissen nach Abschnitt 4.1.4 wurden zehn Querzugversuche am Schraubentyp EJOT JT2-12-5,5x35 V16 durchgeführt.

Die Versuche werden in Anlehnung an den Entwurf von DIN 18807 Teil 4 entsprechend der Darstellung in Bild 4.13 durchgeführt. Die Blechdicken und mechanischen Eigenschaften der Blechstreifen waren die gleichen wie der bei den Schubversuchen eingesetzten Stahlsorten.

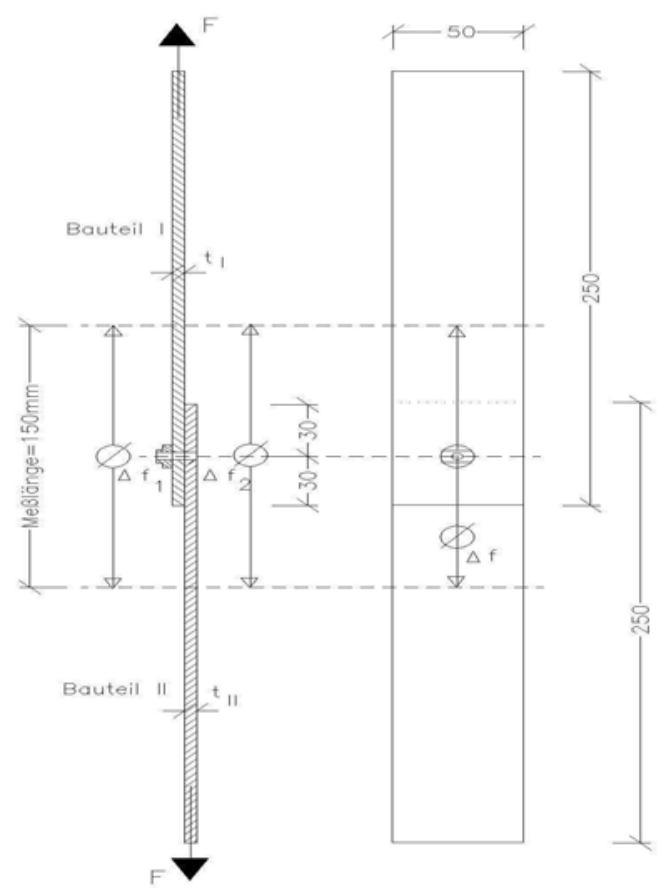


Die Blechstreifen wurden in eine Universalprüfmaschine der Versuchsanstalt eingebaut und mit einer Belastungsgeschwindigkeit von $1,0 \mathrm{~mm} / \mathrm{min}$ gezogen. Die Relativverschiebung zwischen den kraftübertragenden Bauteilen I und II wurde kontinuierlich mit einem Dehnungsaufnehmer gemessen. Sieben Querzugversuche wurden bis zur maximal erreichbaren Relativverschiebung von 3,0 mm geprüft (Last $F_{3,0}$ ), die übrigen wurden zur Ermittlung der maximal möglichen Versagenslast $F_{u}$ bis zum Bruch des Bauteils I geprüft. Sofern sich eine Langlochbildung ergab, wurde diese als Differenz $\Delta$ Loch zwischen den Lochdurchmessern der Bauteile I und II ermittelt. Nach Versuchsende wurde die bleibende Schrägstellung bestimmt.

Die zugehörigen Kraft-Relativverschiebungsdiagramme an den Bohrschrauben des Typs EJOT JT2-12-5,5×35 V16 sind in Bild 4.14 dargestellt. Die Ergebnisse der Querzugversuche sind der Tabelle 4.5 zu entnehmen.

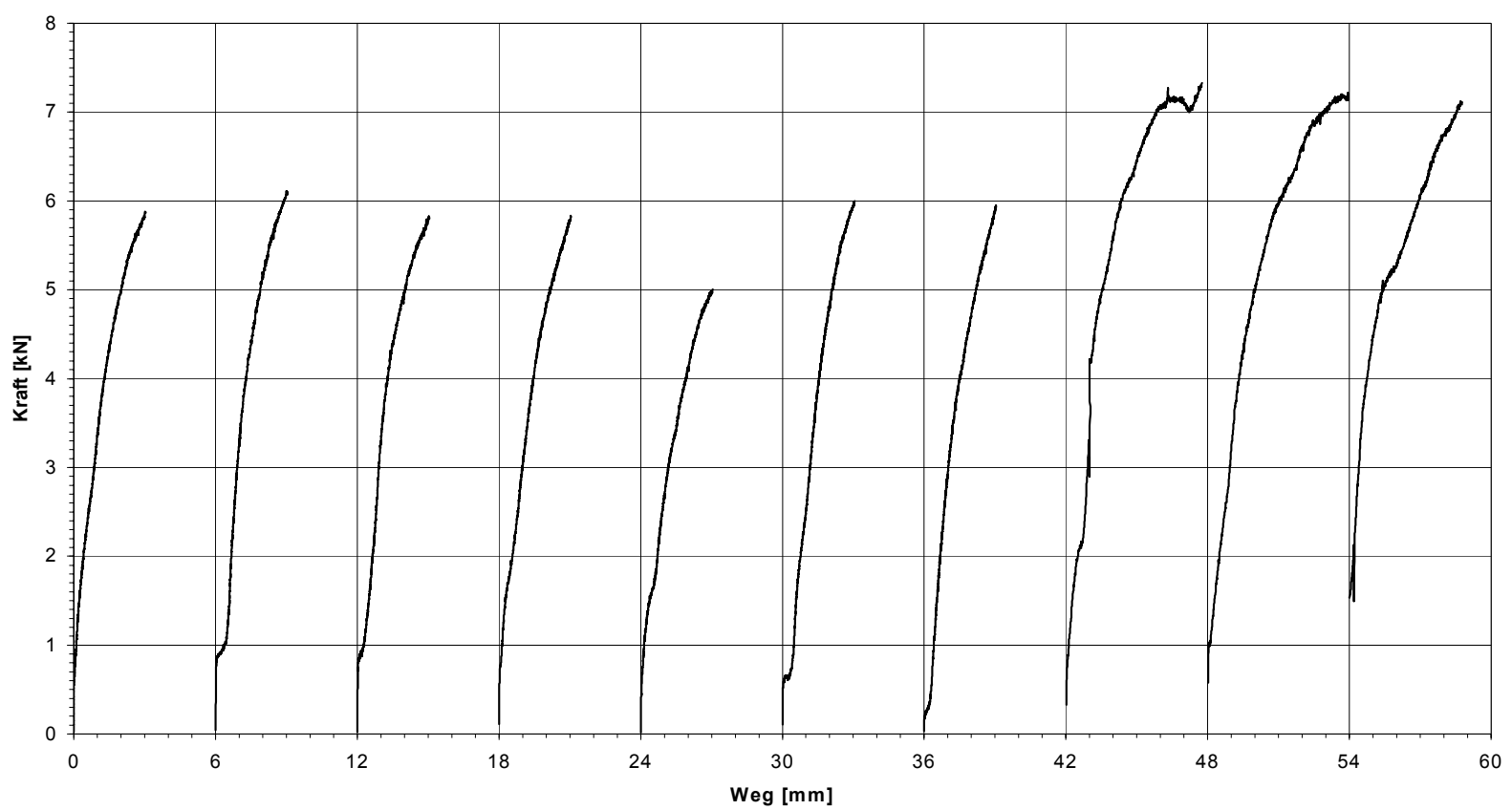

Bild 4.14: Kraft-Relativverschiebungsdiagramme der Querzugversuche an Bohrschrauben EJOT JT2$12-5,5 \times 35$

\begin{tabular}{|c|c|c|c|c|c|c|c|c|}
\hline Versuch $\mathrm{Nr}$. & $\begin{array}{c}\text { Stahlk } \\
\mathrm{t}_{\mathrm{lk}} \\
{[\mathrm{mm}]}\end{array}$ & $\begin{array}{c}\text { ndicken } \\
\qquad \mathrm{t}_{\mathrm{Ilk}} \\
{[\mathrm{mm}]}\end{array}$ & $\begin{array}{l}\mathrm{F}_{3,0} \\
{[\mathrm{kN}]}\end{array}$ & $\begin{array}{c}\mathrm{F}_{\mathrm{u}} \\
{[\mathrm{kN}]}\end{array}$ & $\begin{array}{c}\text { Winkel } \\
{\left[^{\circ}\right]}\end{array}$ & $\begin{array}{c}\Delta \\
\text { Loch } \\
t_{1} \\
{[\mathrm{~mm}]}\end{array}$ & $\begin{array}{c}\Delta \\
\text { Loch } \\
t_{\| 1} \\
{[\mathrm{~mm}]}\end{array}$ & VA \\
\hline QZV-100/800-a- 01 & 0,973 & 8,052 & 5,74 & - & 2 & 2,12 & - & $\mathrm{LI}$ \\
\hline QZV-100/800-a- 02 & 0,964 & 8,107 & 5,75 & - & 0 & 2,04 & - & LI \\
\hline QZV-100/800-a- 03 & 0,958 & 8,102 & 5,58 & - & 1 & 2,22 & - & $\mathrm{LI}$ \\
\hline QZV-100/800-a- 04 & 0,952 & 8,078 & 5,59 & - & 0 & 2,04 & - & $\mathrm{LI}$ \\
\hline QZV-100/800-a- 05 & 0,962 & 8,087 & 4,87 & - & 0 & 2,23 & - & LI \\
\hline QZV-100/800-a- 06 & 0,987 & 8,086 & 5,88 & - & 0 & 2,04 & - & LI \\
\hline QZV-100/800-a- 07 & 0,984 & 8,078 & 5,90 & - & 1 & 2,43 & - & LI \\
\hline QZV-100/800-a- 08 & 0,985 & 8,088 & 6,45 & 7,13 & - & - & - & $\mathrm{BI}$ \\
\hline QZV-100/800-a- 09 & 0,987 & 8,056 & 5,70 & 7,67 & - & - & - & $\mathrm{BI}$ \\
\hline QZV-100/800-a- 10 & 0,977 & 8,122 & 5,58 & 7,53 & - & - & - & $\mathrm{BI}$ \\
\hline VA = Versagensart: & & $\begin{array}{l}\text { LI: } \\
\mathrm{BI}:\end{array}$ & $\begin{array}{l}\text { Versage } \\
\text { Versage }\end{array}$ & $\mathrm{h} \mathrm{Br}$ & $\begin{array}{l}\text { chbildun } \\
\text { im Baute }\end{array}$ & Bautei & & \\
\hline
\end{tabular}

Tabelle 4.5: Ergebnisse der Querzugversuche an Bohrschrauben EJOT JT2-12-5,5x35 


\subsection{Berechnungen mit der Methode der Finiten Elemente}

\subsubsection{Beschreibung des Finite-Element-Modells}

Das Finite-Element-Modell bildet die Trapezprofilscheibe sowie die Unterkonstruktion zur Einleitung der Schubbelastung ab. Der doppeltsymmetrische Aufbau der Trapezprofilscheibe sowie die antimetrische Belastung wurden dazu genutzt, die Berechnungen an einem Viertelmodell durchzuführen. Bild 4.15 zeigt den schematischen Aufbau des Viertelmodells einer zweiseitig gelagerten Trapezprofilscheibe mit dazugehöriger Unterkonstruktion:

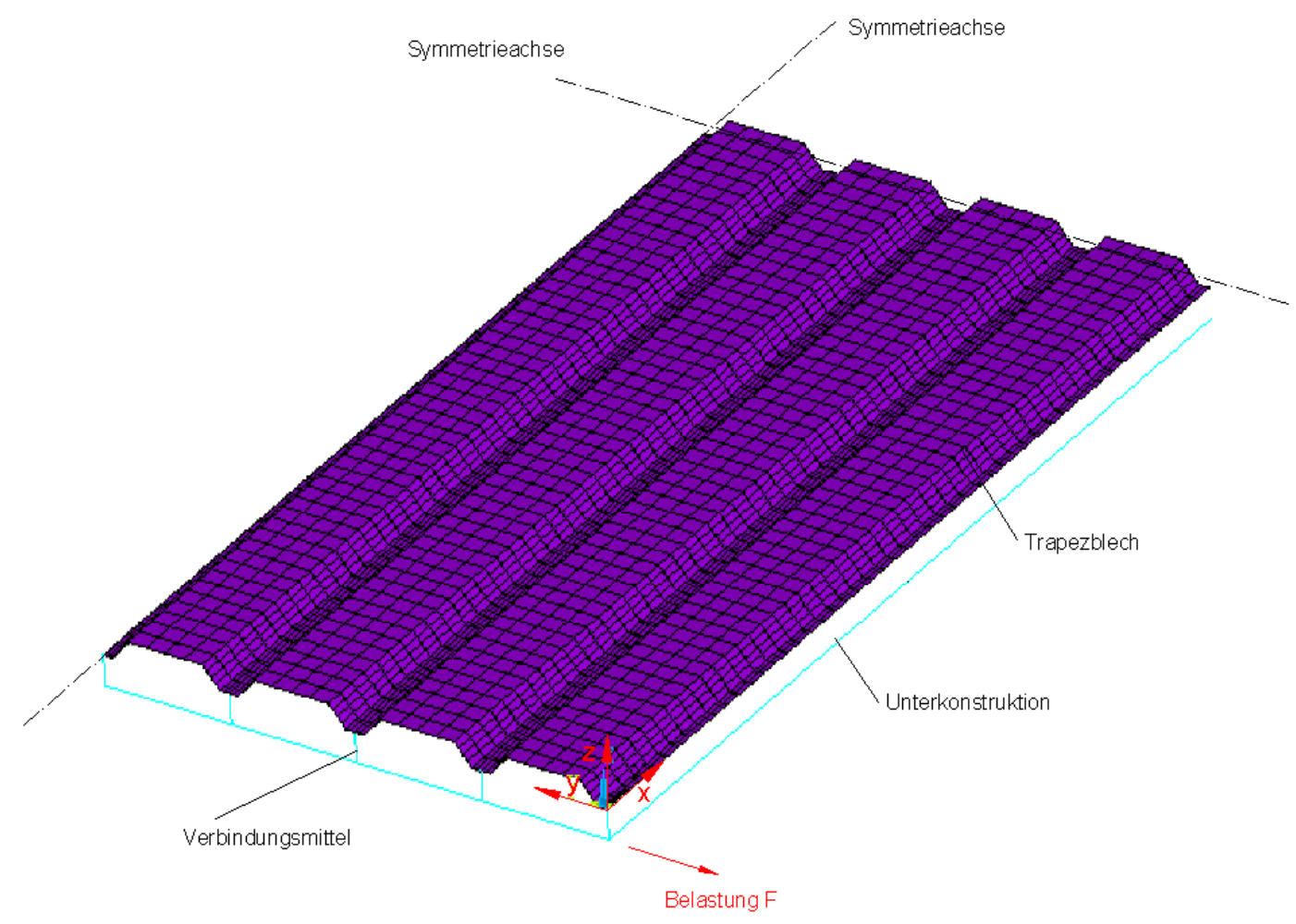

Bild 4.15: Finite-Element-Modellierung der Trapezprofilscheibe mit Unterkonstruktion

Das Trapezblech wird durch Schalenelemente des Typs SHELL181 (vgl. Bild 3.28) abgebildet. Entsprechend Bild 4.15 werden die Unter- und Obergurtbreite jeweils durch eine Mindestanzahl von vier Elementen abgebildet. Die Abbildung der Profilstege erfolgt für die Profilhöhe $35 \mathrm{~mm}$ mit einer Mindestanzahl von vier Elementen. Mit steigender Profilhöhe wird die Elementanzahl linear erhöht. Die Elementierung in x-Richtung erfolgt mit einer Mindestanzahl von 25 Elementen pro Meter Länge.

Die Abbildung der Unterkonstruktion erfolgt durch Balkenelemente des Typs BEAM4, die durch zwei Knoten mit jeweils drei Verschiebungs- und drei Rotationsfreiheitsgraden charakterisiert sind (siehe Bild 4.16). Die zur Berechnung notwendigen Querschnittswerte der Balkenelemente werden vom Benutzer eingegeben. Die Umsetzung der gelenkigen Verbindung in den Eckpunkten der Unterkonstruktion erfolgt durch translatorische Kopplung der betreffenden Knoten. 


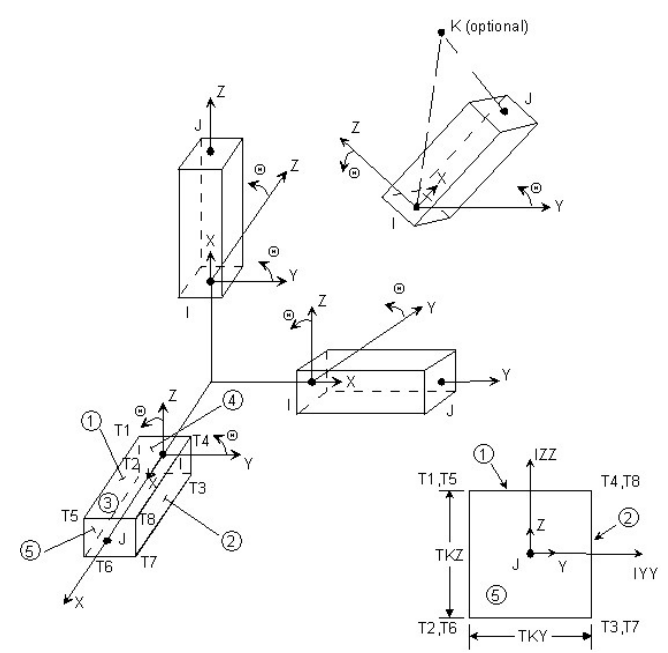

Bild 4.16: Elementtyp BEAM4 [1]

Für beide Elementtypen (Schalen- und Balkenelemente) wird ein linear-elastisches, ideal-plastisches Werkstoffgesetz angesetzt, wobei nach Überschreiten der Streckgrenze $f_{y}$ Fließen ohne Verfestigung einsetzt.

Die Verbindung zwischen den einzelnen Trapezprofiltafeln sowie zwischen Trapezprofil und Unterkonstruktion wird durch das nichtlineare Federelement COMBIN39 (siehe Bild 4.17) abgebildet. Dieses Element wird durch zwei Knoten mit bis zu drei Verschiebungsfreiheitsgraden sowie der Eingabe eines Last-Verformungsverhaltens in Form eines Linienzugs definiert. Hierbei kann vom Anwender sowohl ein nichtlineares Last-Verformungsverhalten als auch ein individuelles Entlastungsverhalten definiert werden. Für die nachfolgende Verifizierung des Finite-Element-Modells mit den Versuchsergebnissen wird das durch Versuche ermittelte nichtlineare Federgesetz angesetzt. Für die anschließende parametrische Untersuchung wird ein lineares Last-Verformungsverhalten gewählt. Eventuell vorhandene Reibeinflüsse im Bereich der Verbindungsmittel wurden in allen Fällen vernachlässigt.

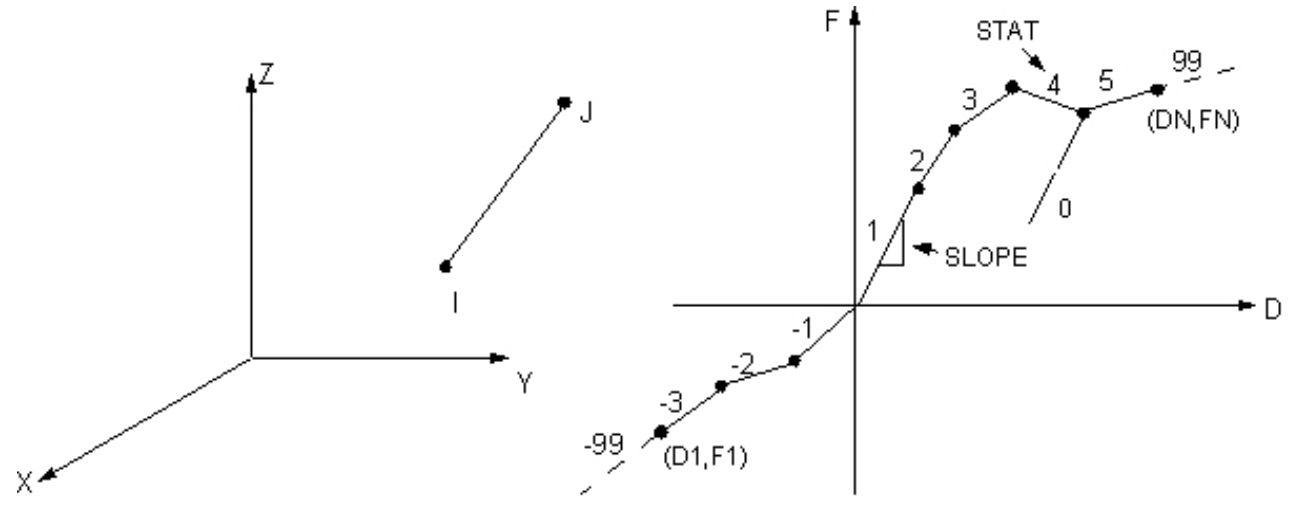

Bild 4.17: Elementtyp COMBIN39 mit frei wählbarem Last-Verformungsverhalten [1]

Entsprechend Bild 4.18 ergeben sich nach DIN 18807 Teil 1 infolge der Schubfeldwirkung an den Querrändern abhebende und andrückende Kontaktkräfte $R_{S}$. Aus den abhebenden Kräften entstehen zusätzliche Beanspruchungen für die Verbindungsmittel, während die andrückenden Kräfte eine Zusatzbeanspruchung für den Profilsteg darstellen. Dieser Sachverhalt wurde im Finite-Element-Modell durch Ansatz eines Festlagers an der andrückenden Seite berücksichtigt, während die abhe- 
bende Seite in ihrer Bewegungsfreiheit nur durch die benachbarten Verbindungsmittel eingeschränkt war.
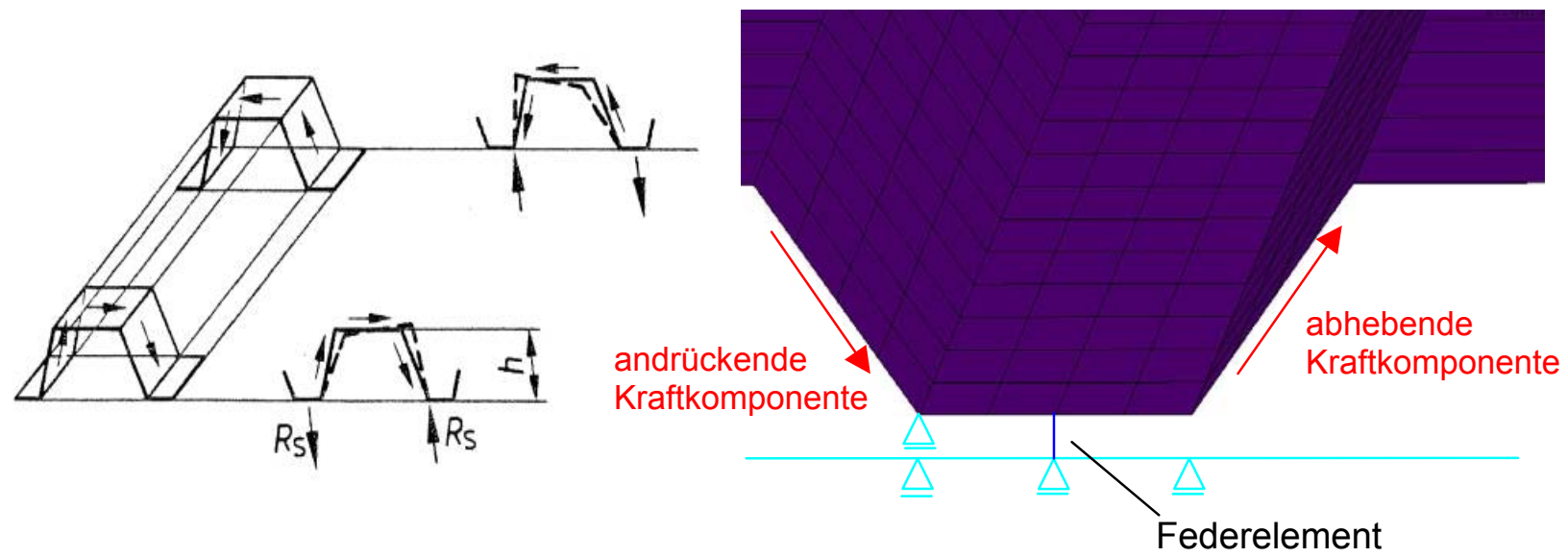

Bild 4.18: Kontaktkräfte infolge Schubfeldwirkung und Umsetzung im FE-Modell

\subsubsection{Nachgiebigkeit der Verbindungsmittel}

Das Last-Verformungsverhalten im Querzugversuch wird durch das Verbindungsmittel, die Blechdicken sowie die Zugfestigkeiten der miteinander verbundenen Blechteile beeinflusst. Für unterschiedliche Typen von Verbindungsmitteln werden in [19] experimentell ermittelte Nachgiebigkeiten sowie Formeln für die Berechnung der Grenzlochleibungskräfte angegeben. Die Berechnung der Grenzlochleibungskraft erfolgt dabei in Anlehnung an DIN EN 1993-1-3, wobei der Teilsicherheitsbeiwert wegen der großen Anzahl der Verbindungsmittel in einem Schubfeld mit 1,1 statt wie sonst üblich mit 1,25 angesetzt wird. Eine Zusammenstellung dieser Angaben ist Tabelle 4.6 zu entnehmen.

\begin{tabular}{|c|c|c|c|c|c|}
\hline $\begin{array}{l}\text { Befesti- } \\
\text { gungsart }\end{array}$ & Typ & $\begin{array}{l}\text { Dicht- } \\
\text { scheibe }\end{array}$ & $\begin{array}{c}\text { Durchmesser } \\
{[\mathrm{mm}]}\end{array}$ & $\begin{array}{l}\text { Bemessungsquerkraft aus Lochleibung } \\
\qquad[\mathrm{kN}]\end{array}$ & $\begin{array}{l}\text { Nachgiebig- } \\
\text { keit } \mathrm{s}_{\mathrm{p}} \\
{[\mathrm{mm} / \mathrm{kN}]}\end{array}$ \\
\hline \multirow{4}{*}{$\begin{array}{l}\text { Querrand- } \\
\text { oder } \\
\text { Längrand- } \\
\text { befestigung }\end{array}$} & \multirow{4}{*}{ Schrauben } & \multirow{2}{*}{ ohne } & 5,5 & $F_{s c}=1,9 \cdot f_{u} \cdot d_{n} \cdot t_{K} \leq 6,5$ & \multirow{2}{*}{0,15} \\
\hline & & & 6,3 & $F_{s c}=1,9 \cdot f_{u} \cdot d_{n} \cdot t_{K} \leq 8,0$ & \\
\hline & & \multirow{2}{*}{$\begin{array}{l}\text { EPDM- } \\
\text { Dicht- } \\
\text { scheibe }\end{array}$} & 5,5 & $F_{s c}=1,9 \cdot f_{u} \cdot d_{n} \cdot t_{K} \leq 6,5$ & \multirow{2}{*}{0,35} \\
\hline & & & 6,3 & $F_{s c}=1,9 \cdot f_{u} \cdot d_{n} \cdot t_{K} \leq 8,0$ & \\
\hline $\begin{array}{c}\quad \text { mit } \\
t_{I I} \geq 2,5 t_{l}\end{array}$ & Setzbolzen & $\begin{array}{l}\text { Stahl- } \\
\text { scheibe } \\
\varnothing 23 \mathrm{~mm}\end{array}$ & $3,7-4,8$ & $F_{p}=2,9 \cdot f_{u} \cdot d_{n} \cdot t_{K} \leq 8,0$ & 0,10 \\
\hline $\begin{array}{l}\text { Befesti- } \\
\text { gungsart }\end{array}$ & Typ & $\begin{array}{l}\text { Dicht- } \\
\text { scheibe }\end{array}$ & $\begin{array}{c}\text { Durchmesser } \\
{[\mathrm{mm}]}\end{array}$ & $\begin{array}{l}\text { Bemessungsquerkraft aus Lochleibung } \\
\qquad[\mathrm{kN}] \\
\end{array}$ & $\begin{array}{c}\text { Nachgiebig- } \\
\text { keit } \mathrm{s}_{\mathrm{s}} \\
{[\mathrm{mm} / \mathrm{kN}]} \\
\end{array}$ \\
\hline \multirow{2}{*}{$\begin{array}{l}\text { Längsstoß- } \\
\text { verbinder } \\
\text { mit } t_{\| 1}=t_{1}\end{array}$} & Schrauben & ohne & $4,1-4,8$ & $F_{S}=2,9 \cdot\left(t_{K} / d_{n}\right)^{\frac{1}{2}} f_{u} \cdot d_{n} \cdot t_{K} \leq 3,8$ & 0,25 \\
\hline & Nieten & - & 4,8 & $F_{S}=3,2 \cdot\left(t_{K} / d_{n}\right)^{\frac{1}{2}} f_{u} \cdot d_{n} \cdot t_{K} \leq 3,0$ & 0,30 \\
\hline mit & $\begin{array}{l}f_{u} \\
d_{n} \\
t_{k}\end{array}$ & & & $\begin{array}{l}\mathrm{N} / \mathrm{mm}^{2} \text { in }\left[\mathrm{kN} / \mathrm{mm}^{2}\right] \\
\text { /erbindungsmittels [mm] } \\
\text { in }[\mathrm{mm}]\end{array}$ & \\
\hline
\end{tabular}

Tabelle 4.6: Bemessungskräfte und Nachgiebigkeiten nach [19] für unterschiedliche Befestigungen 
Im Hinblick auf die Untersuchungen der nachfolgenden Abschnitte werden die in Tabelle 4.6 für Quer- oder Längsrandbefestigung aufgeführten Nachgiebigkeiten bis zu dem Niveau der Bemessungswerte der Querkrafttragfähigkeit $V_{R, d}$ aus [14] angesetzt. Aus vereinfachenden Gründen werden alle Längsstoßverbindungen (Schrauben und Niete) mit einer Nachgiebigkeit $s_{s}=0,30 \mathrm{~mm} / \mathrm{kN}$ ebenfalls bis zu dem Niveau der Bemessungswerte der Querkrafttragfähigkeit $V_{R, d}$ angesetzt.

Die Gültigkeit der Nachgiebigkeit für Setzbolzen wird durch [3] bestätigt, wo LastRelativverschiebungsdiagramme aus Querzugversuchen für unterschiedliche Blechdickenkombinationen angegeben sind. Danach ist der Ansatz einer Nachgiebigkeit $\mathrm{s}_{\mathrm{p}}=0,10 \mathrm{~mm} / \mathrm{kN}$ entsprechend Tabelle 4.6 gerechtfertigt.

\subsubsection{Verifizierung der Finite-Element-Modells mit Berechnungsergebnissen}

Die Verifizierung des in Abschnitt 4.2.1 beschriebenen Finite-Element-Modells erfolgt durch Vergleich mit nach [53] berechneten Kennwerten. Hierzu wurden die drei in Bild 4.19 dargestellten Querschnittsgeometrien handelsüblicher Trapezprofile modelliert und mit jeweils drei unterschiedlichen Blechdicken bei vierseitiger Lagerung berechnet.

Profil 35/207:

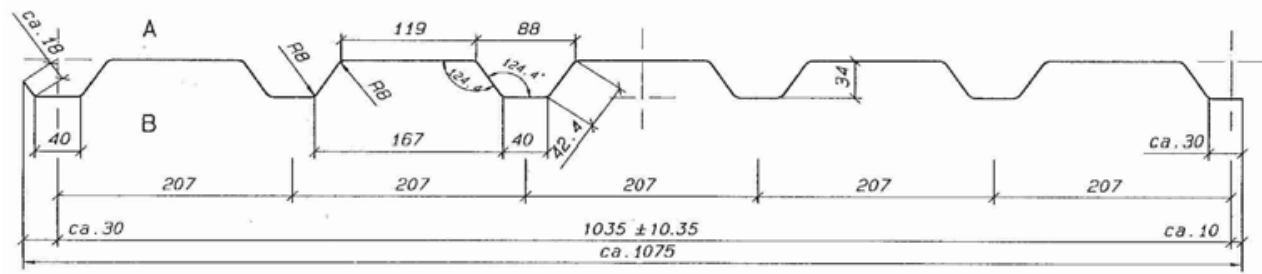

Profil 105/345:

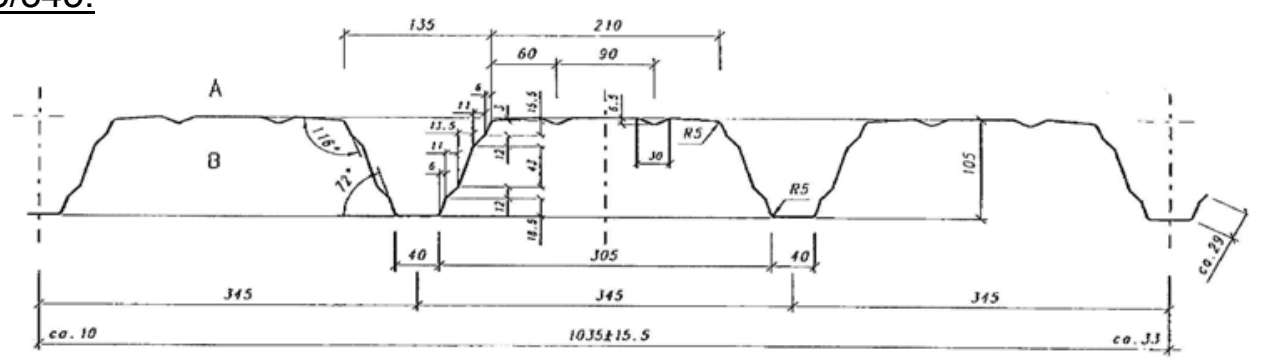

Profil 135/310:

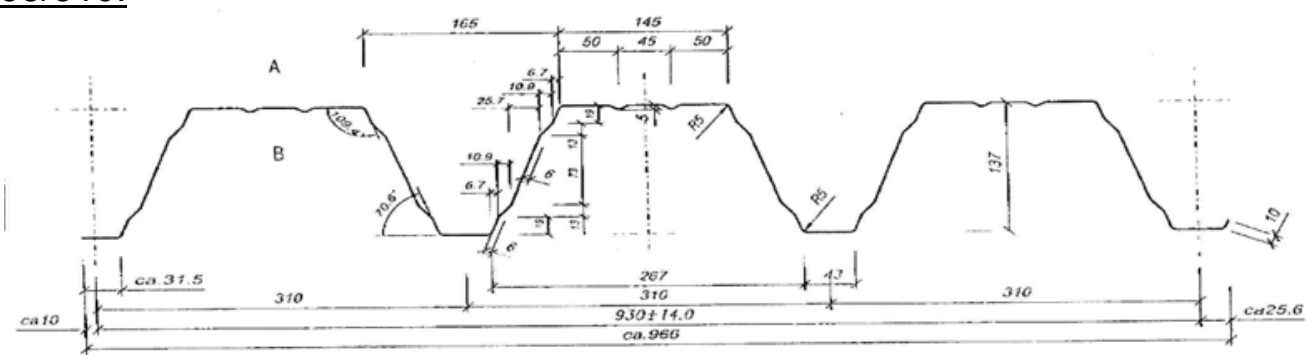

Bild 4.19: untersuchte Profilgeometrien

Entsprechend der [2], [52] und [53] zugrunde liegenden Elastizitätstheorie wurden die Vergleichsberechnungen mit einem ideal-elastischen Werkstoffgesetz mit einem 
Elastizitätsmodul $E=210000 \mathrm{~N} / \mathrm{mm}^{2}$ und einer Querkontraktionszahl $\mu=0,3$ durchgeführt. Da [2], [52] und [53] weder Nachgiebigkeiten der Verbindungsmittel noch Verformungen der Unterkonstruktion berücksichtigen, wurden die Federelemente des Finite-Element-Modells durch starre Kopplungen ersetzt und die Balkenelemente der Unterkonstruktion mit einer unendlich großen Biege- und Dehnsteifigkeit versehen. Stegversätze und Gurtsicken wurden in der Finite-Element-Modellierung nicht berücksichtigt. Aus der mit diesen Annahmen ermittelten Schubfeldverformung wurde entsprechend Gleichung (4.2) die Schubfeldsteifigkeit $S$ berechnet, die in Tabelle 4.7 den Steifigkeiten nach [53] gegenübergestellt ist.

\begin{tabular}{|c|c|c|c|c|c|}
\hline \multirow{2}{*}{$\begin{array}{c}\text { Profil- } \\
\text { bezeichung }\end{array}$} & \multirow{2}{*}{$\begin{array}{l}\text { Schubfeld- } \\
\text { länge } I_{S}[\mathrm{~m}]\end{array}$} & \multirow{2}{*}{$\begin{array}{c}\text { Blechdicke } \\
\text { [mm] }\end{array}$} & \multicolumn{2}{|c|}{ Schubsteifigkeiten S [kN/m] } & \multirow{2}{*}{$\begin{array}{c}\text { Abweichung } \\
{[\%]}\end{array}$} \\
\hline & & & FE-Berechnung & [53] & \\
\hline \multirow{3}{*}{$35 / 207$} & \multirow{3}{*}{3,0} & 0,75 & 4215 & 4366 & 4 \\
\hline & & 1,00 & 8621 & 8831 & 2 \\
\hline & & 1,25 & 15540 & 14926 & 4 \\
\hline \multirow{3}{*}{$105 / 345$} & \multirow{3}{*}{6,0} & 0,75 & 807 & 794 & 2 \\
\hline & & 1,00 & 1727 & 1669 & 3 \\
\hline & & 1,25 & 3039 & 2941 & 3 \\
\hline \multirow{3}{*}{$135 / 310$} & \multirow{3}{*}{6,0} & 0,75 & 1053 & 1103 & 5 \\
\hline & & 1,00 & 2198 & 2306 & 5 \\
\hline & & 1,25 & 3829 & 4036 & 5 \\
\hline
\end{tabular}

Tabelle 4.7: Vergleich der Schubsteifigkeiten nach Gleichung (4.2) mit FEM und [53]

Die geringen Abweichungen zeigen, dass mit dem Finite-Element-Modell die Schubsteifigkeiten eines vierseitig gelagerten Schubfeldes aus Trapezprofilen berechnet werden können. In [46] wurden mit dem Finite-Element-Modell sowohl Horizontalund Vertikalverschiebungen charakteristischer Punkte der Profilrippen als auch Querbiegespannungen infolge Schubbeanspruchung ermittelt und mit den Angaben aus [52] verglichen. Der Vergleich zeigte eine gute Übereinstimmung und bestätigt die Eignung des Finite-Element-Modells zur Berechnung von vierseitig gelagerten Trapezprofilen unter Schubbeanspruchung.

\subsubsection{Verifizierung des Finite-Element-Modells mit Versuchsergebnissen}

Mit dem Finite-Element-Modell wurden Berechnungen zum Vergleich mit den unter Abschnitt 4.1 beschriebenen Schubfeldversuchen am Profil LS 5/35/1035 durchgeführt. Der Vergleich erfolgt hinsichtlich der Schubfeldverformung $v_{S}$, der Schubfeldsteifigkeit $S$ und der Traglast $F_{u}$.

Der Einfluss der Nachgiebigkeit der Verbindungsmittel am Quer- und gegebenenfalls auch am Längsrand wird dabei durch die Federelemente berücksichtigt. Dazu werden die unter Abschnitt 4.1.5 experimentell ermittelten Last-Verformungskurven entsprechend Bild 4.20 durch einen nichtlinearen Linienzug angenähert und den Federelementen zugewiesen. Den Schalenelementen zur Abbildung des Trapezblechs wurden die in Abschnitt 4.1.2 ermittelten Werte von Kernblechdicke und Streckgrenze zugrunde gelegt. 


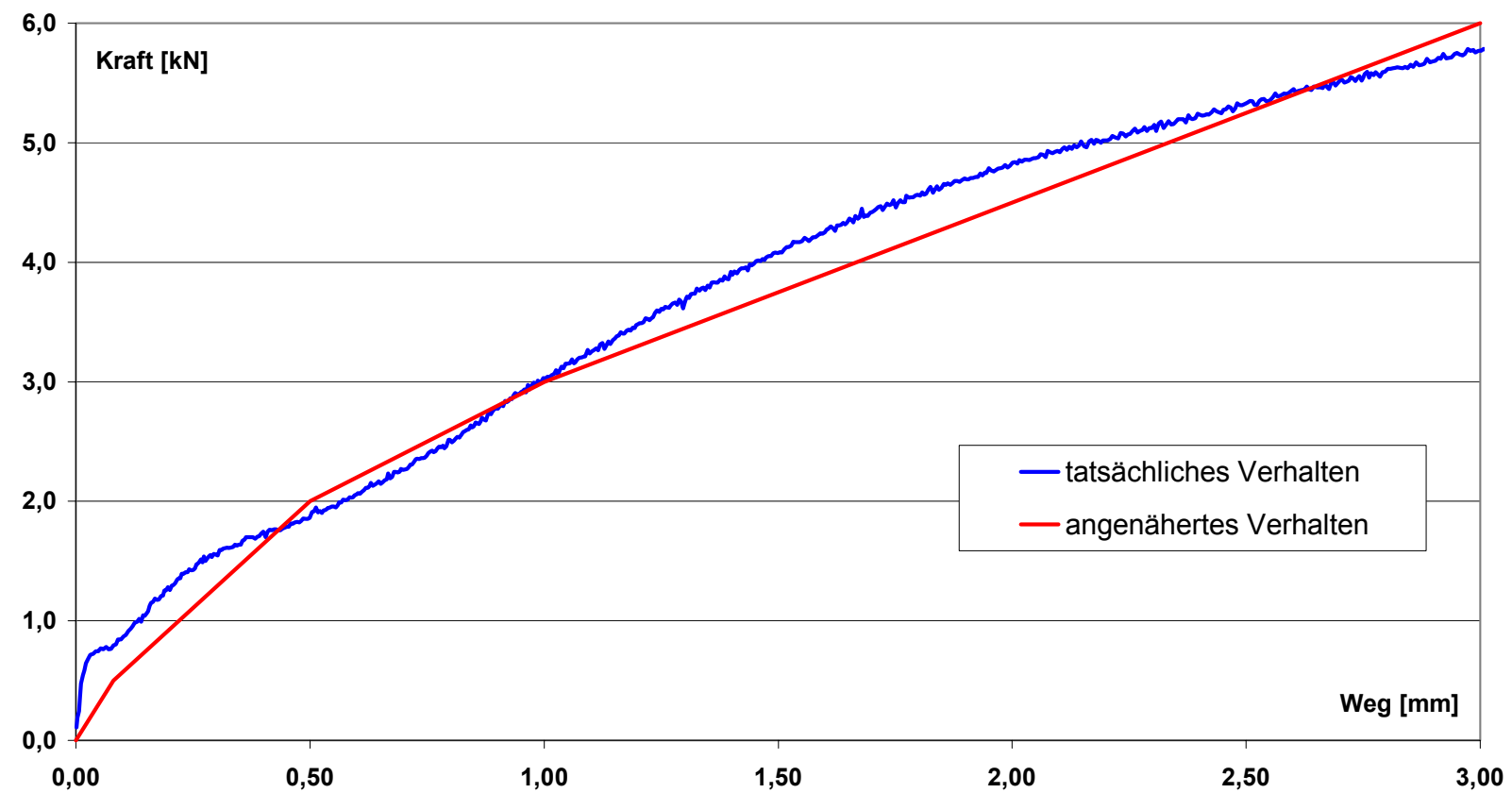

Bild 4.20: idealisiertes Last-Verformungsdiagramm der Bohrschrauben EJOT JT2-12-5,5x35

Für die vierseitige Lagerung ist in Bild 4.21 die Schubfeldverformung $v_{s}$ nach der Finite-Element-Berechnung der experimentell bestimmten Last-Verformungs-Kurve gegenübergestellt.

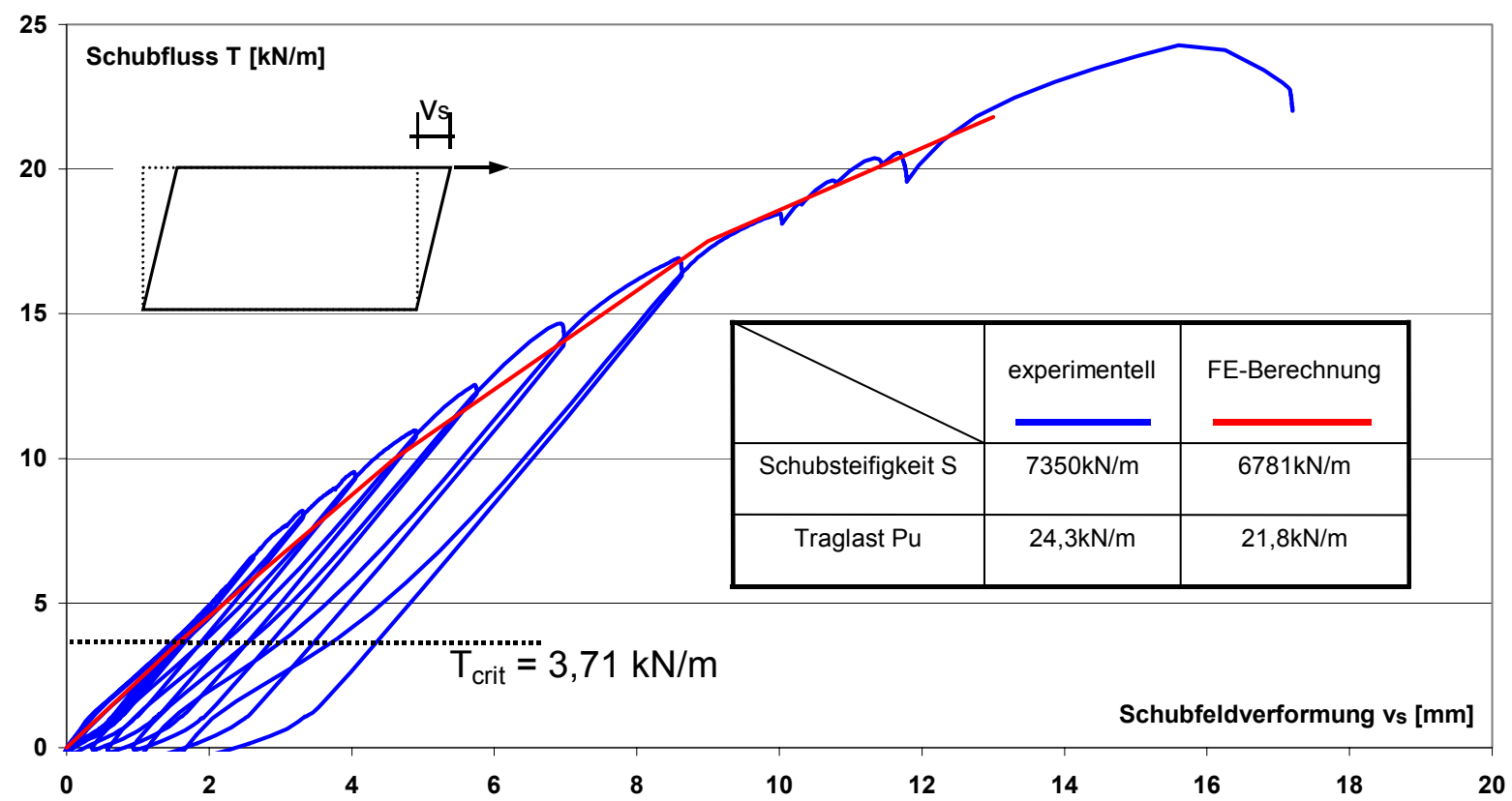

Bild 4.21: Vergleich der Last-Verformungsbeziehung nach experimenteller Untersuchung und FEM für vierseitige Lagerung

Das Diagramm zeigt einen nahezu gleichen Verlauf beider Kurven. Die Auswertung der Versuchskurven hinsichtlich der Schubsteifigkeit S erfolgt beim kritischen Schubfluss $T_{\text {crit }}=3,71 \mathrm{kN} / \mathrm{m}$ (vgl. Abschnitt 4.1.4). Dieser Wert liegt innerhalb des linearen Anfangsbereichs, der bei der experimentellen Untersuchung bis zu einem Schubfluss von $8,5 \mathrm{kN} / \mathrm{m}$ reicht. Bei der Finite-Element-Berechnung deuten sich erste Steifigkeitsänderungen im Last-Verformungsdiagramm aufgrund der im System vorhandenen Nichtlinearitäten von Material und Verhalten der Verbindungsmittel ab einem 
Wert von etwa $6,5 \mathrm{kN} / \mathrm{m}$ an. Die Auswertung ergibt die in Bild 4.21 aufgeführten Zahlenwerte. Der Unterschied zur experimentellen Untersuchung beträgt $8 \%$ bei der Schubsteifigkeit und $11 \%$ bei der Traglast. Diese war sowohl bei der experimentellen als auch der analytischen Untersuchung durch die maximal aufnehmbare Schraubenkraft bestimmt. In der Finite-Element-Berechnung wurde dieser Sachverhalt durch ein Abbruchkriterium bei Erreichen der zulässigen Schraubenkraft definiert. In beiderlei Hinsicht (Schubsteifigkeit und Traglast) stimmen experimentelle und analytische Untersuchung gut überein.

Für die zweiseitige Lagerung ist in Bild 4.22 die Schubfeldverformung $v_{s}$ nach der Finite-Element-Berechnung der experimentell bestimmten Last-Verformungs-Kurve gegenübergestellt.

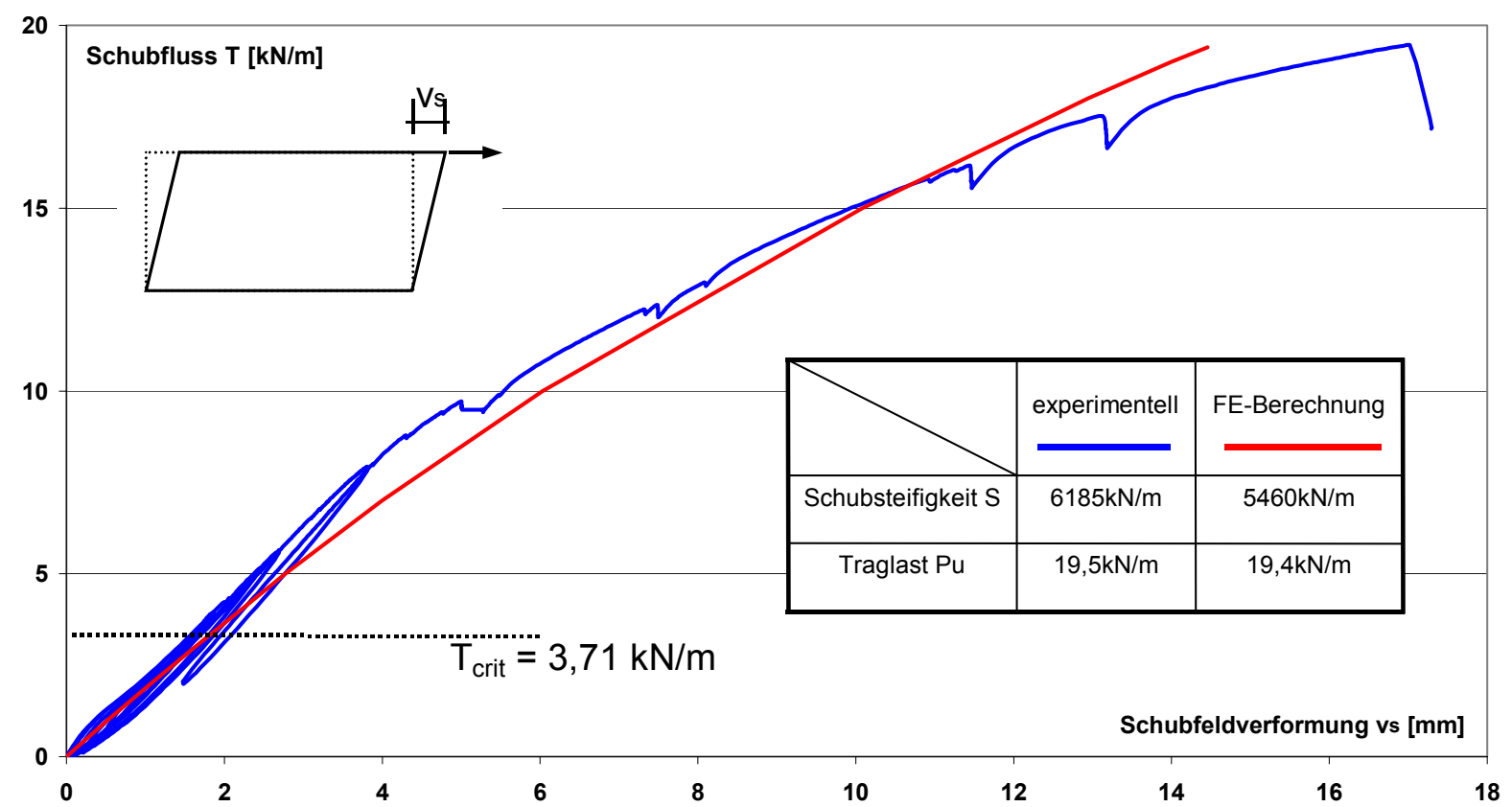

Bild 4.22: Vergleich der Last-Verformungsbeziehung nach experimenteller Untersuchung und FEM für zweiseitige Lagerung

Beide Kurven haben nahezu den gleichen Verlauf. Nach einer sehr guten Übereinstimmung der Verläufe im Anfangsbereich bis zu einem Schubfluss von $5,0 \mathrm{kN} / \mathrm{m}$ sind die Verschiebungen nach der Finite-Element-Berechnung im Bereich zwischen $5,0 \mathrm{kN} / \mathrm{m}$ und $15 \mathrm{kN} / \mathrm{m}$ geringfügig größer. Dies folgt daraus, dass in der Finite-Element-Berechnung bereits bei $5,0 \mathrm{kN} / \mathrm{m}$ die Steifigkeit abnimmt, während dies bei der experimentellen Untersuchung erst bei etwa $8,0 \mathrm{kN} / \mathrm{m}$ auftritt. Die Auswertung der Daten ergibt die in Bild 4.22 aufgeführten Zahlenwerte. Der Unterschied zur experimentellen Untersuchung beträgt $13 \%$ bei der Schubsteifigkeit und $1 \%$ bei der Traglast, die sowohl bei der experimentellen als auch der analytischen Untersuchung durch die maximal aufnehmbare Schraubenkraft bestimmt war. In beiderlei Hinsicht (Schubsteifigkeit und Traglast) stimmen experimentelle und numerische Untersuchung gut überein.

Bemerkenswert ist die Tatsache, dass die Traglast im Fall der vierseitigen Lagerung etwa um den Faktor 6, im Fall der zweiseitigen Lagerung etwa um den Faktor 5 über dem kritischen Schubfluss $T_{\text {crit }}$ liegt. 


\subsubsection{Berechnungen mit dem verifizierten Finite-Element-Modell zur Bestim- mung der signifikanten Einflüsse}

\subsubsection{Allgemeines}

Die Geringfügigkeit der Unterschiede, die sich bei der Verifizierung des FiniteElement-Modells in Abschnitt 4.2.3 und in Abschnitt 4.2.4 ergibt, zeigt, dass das Finite-Element-Modell für die Untersuchung von Schubfeldern aus Trapezprofilen gut geeignet ist.

In den folgenden Abschnitten wird der Einfluss der Systemparameter auf die gemäß Gleichung (4.2) definierte Schubsteifigkeit untersucht, um die signifikanten Einflüsse im Hinblick auf das Schubtragverhalten eines zweiseitig gelagerten Schubfeldes festzustellen.

\subsubsection{Berücksichtigung unterschiedlicher Profiltypen}

Die parametrischen Berechnungen wurden mit verschiedenen Profilgeometrien durchgeführt. Die Profiltypen wurden aus handelsüblichen Trapezprofilen (zum Beispiel [39]) so ausgewählt, dass mit Profilhöhen von $30 \mathrm{~mm}$ bis $159 \mathrm{~mm}$ ein möglichst breiter Bereich der Schubsteifigkeit abgedeckt war, und schloss das in Abschnitt 4.1 untersuchte Profil LS 5/35/1035 mit ein. Bei diesem Profil wurden die gemessene Profilgeometrie sowie die ermittelten Werkstoffkennwerte zugrunde gelegt. Die bei den anderen Profilen zur Berechnung verwendeten nominellen Querschnittsabmessungen, Kernblechdicken und Schubfeldlängen sind in Tabelle 4.8 angegeben. Die Schubsteifigkeit $S$ wurde für jedes Profil beim jeweils kritischen Schubfluss $T_{\text {crit }}$ nach [53] ermittelt. Die Berechnungen wurden mit einem bilinearen Werkstoffgesetz unter Berücksichtigung der Parameter $E=210000 \mathrm{~N} / \mathrm{mm}^{2}$ und $f_{y}=320 \mathrm{~N} / \mathrm{mm}^{2}$ durchgeführt.

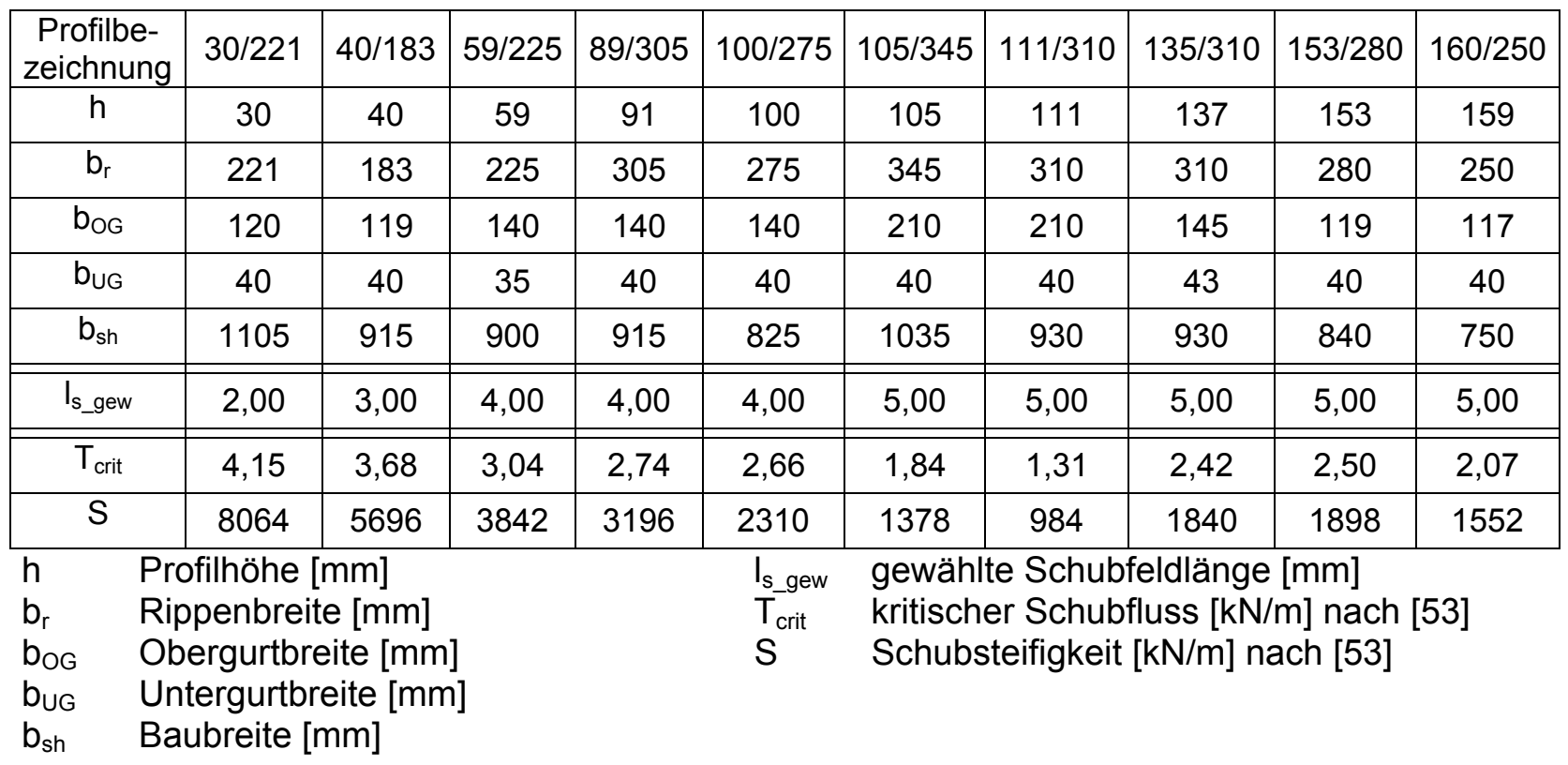

Tabelle 4.8: Zusammenstellung der untersuchten Profiltypen 


\subsubsection{Einfluss der Nachgiebigkeit der Verbindungsmittel an den Schubfeld- rändern bei zwei- und vierseitiger Lagerung}

Nachgiebigkeiten in der Befestigung der Trapezprofile auf der Unterkonstruktion führen zu zusätzlichen Schubfeldverformungen, die die vorhandene Schubsteifigkeit mindern. Der Einfluss der in Abschnitt 4.1.5 ermittelten und in Tabelle 4.6 aufgeführten Nachgiebigkeiten für unterschiedliche Längs- und Querrandbefestigungen wird durch die nachfolgenden Finite-Element-Berechnungen an zweiseitig und vierseitig gelagerten Trapezprofilscheiben mit Seitenverhältnissen $\alpha$ gemäß Gleichung (4.6) untersucht.

$$
\alpha=\frac{a}{b}=\frac{a}{n_{s h} \cdot b_{s h}}
$$

$\begin{array}{lll}\text { mit } & a & \text { Schubfeldlänge } \\ & \mathrm{b} & \text { Schubfeldbreite } \\ & \mathrm{n}_{\mathrm{sh}} & \text { Anzahl Profiltafeln } \\ & \mathrm{b}_{\mathrm{sh}} & \text { Baubreite der Profiltafeln }\end{array}$

Der Einfluss von nachgiebigen Verbindungsmitteln auf die Schubsteifigkeit eines vierseitig gelagerten Schubfeldes kann entsprechend Gleichung (4.7) als ein System zweier hintereinander geschalteter Federn dargestellt werden.

$$
\frac{1}{\mathrm{~S}_{4 \_ \text {flex }}}=\frac{1}{\mathrm{~S}_{4 \_ \text {inflex }}}+\frac{1}{\mathrm{~S}_{\text {fast }}}
$$

mit $\quad S_{4}$ flex $\quad$ Schubsteifigkeit mit nachgiebigen Verbindungsmitteln

$\mathrm{S}_{4 \_ \text {_inflex }} \quad$ Schubsteifigkeit nach [53] (unnachgiebige Verbindungsmittel)

$S_{\text {fast }} \quad$ Schubsteifigkeit der nachgiebigen Verbindungsmittel

Aus Gleichung (4.7) folgt die Darstellung für die Schubsteifigkeit mit nachgiebigen Verbindungsmitteln $\mathrm{S}_{4}$ flex $\mathrm{zu}$

$$
\frac{1}{S_{4 \_ \text {flex }}}=\frac{S_{4 \_ \text {inflex }} \cdot S_{\text {fast }}}{S_{4 \_ \text {inflex }}+S_{\text {fast }}}=S_{4 \_ \text {inf lex }} \cdot \frac{1}{1+\frac{S_{4 \_ \text {inflex }}}{S_{\text {fast }}}}
$$

womit das Verhältnis $S_{4 \_f l e x} z u S_{4 \_ \text {inflex }}$ über den Verhältniswert $\kappa$ entsprechend Gleichung (4.9)

dargestellt werden kann.

$$
\kappa=\frac{\mathrm{S}_{4 \_ \text {flex }}}{\mathrm{S}_{4 \_ \text {inflex }}}=\frac{1}{1+\frac{\mathrm{S}_{4 \_ \text {inflex }}}{\mathrm{S}_{\text {fast }}}}
$$

Die in Bild 4.23 für die Profiltypen aus Tabelle 4.8 wiedergegebenen Berechnungsergebnisse zeigen den Einfluss der Verbindungsmittelnachgiebigkeiten für eine vierseitig gelagerte Trapezprofilscheibe mit einem Seitenverhältnis $\alpha \approx 1,0$ als Verhältniswerte $\kappa$ nach Gleichung (4.9). Zusätzlich zu den in Tabelle 4.6 aufgeführten Nachgiebigkeiten $\left(s_{p}=0,10 \mathrm{~mm} / \mathrm{kN}, s_{p}=0,15 \mathrm{~mm} / \mathrm{kN}, s_{p}=0,35 \mathrm{~mm} / \mathrm{kN}\right)$ wurde auch die Nachgiebigkeit $s_{p}=0,50 \mathrm{~mm} / \mathrm{kN}$ für Befestigungsarten, die die Kriterien nach Tabelle 4.6 nicht erfüllen, und eine unnachgiebige Lagerung (näherungsweise mit 
$\mathrm{s}_{\mathrm{p}}=0,0001 \mathrm{~mm} / \mathrm{kN}$ ) untersucht. Die Verbindungsmittel am Längsrand waren im Abstand von $50 \mathrm{~mm}$ angeordnet.

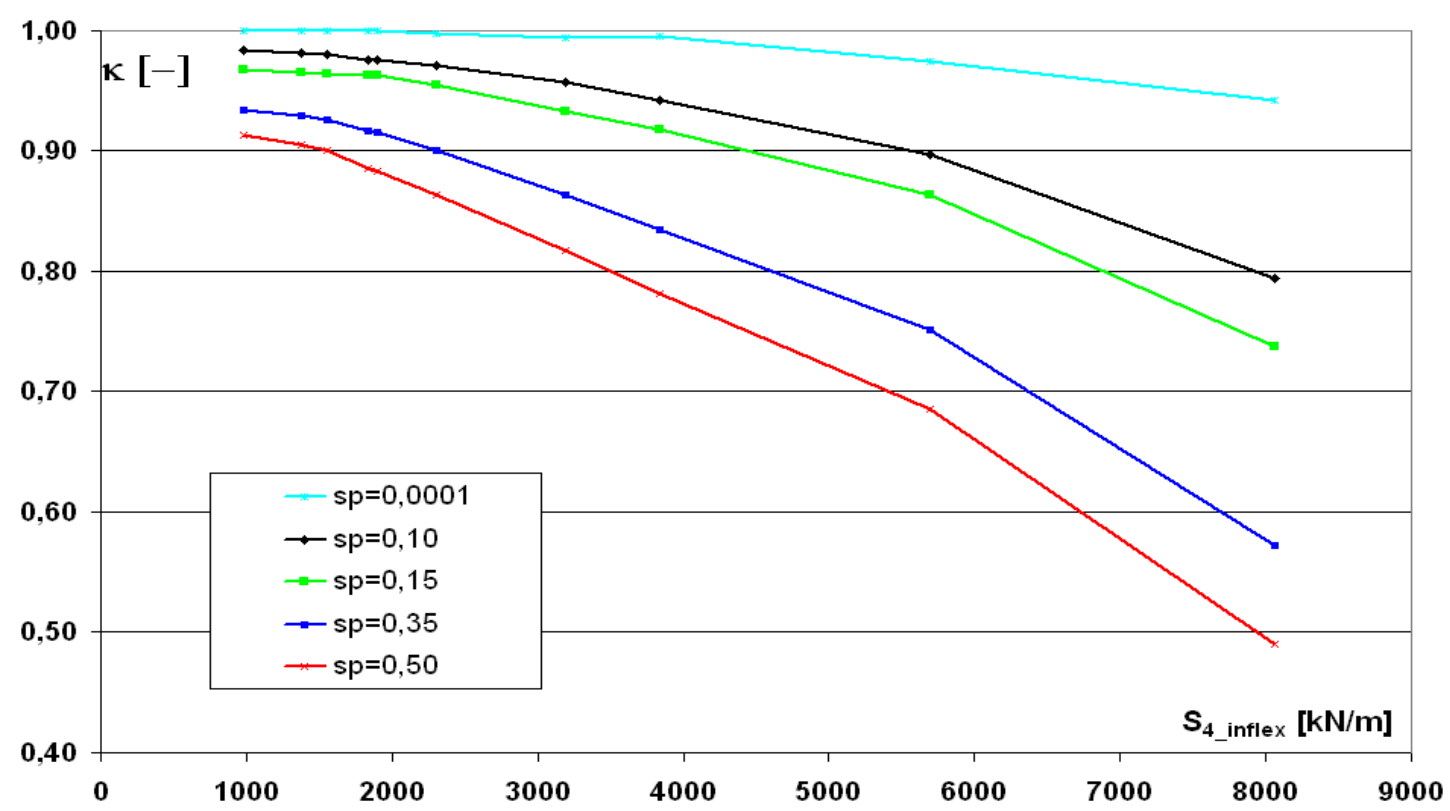

Bild 4.23: Verhältniswerte $\kappa$ für ein Seitenverhältnis des vierseitig gelagerten Schubfeldes von $\alpha \approx 1,0$

Die Kurvenverläufe in Bild 4.23 zeigen einen mit der Schubsteifigkeit $\mathrm{S}_{4 \text { _inflex }}$ zunehmenden Einfluss der Nachgiebigkeiten. Die zugehörigen Verhältniswerte $\kappa$ können für den Fall der Nachgiebigkeiten aus Tabelle 4.6 bis auf 0,80 für $s_{p}=0,10 \mathrm{~mm} / \mathrm{kN}$ und 0,58 für $s_{p}=0,35 \mathrm{~mm} / \mathrm{kN}$ abfallen. Bei den schubweichen Profilen mit großer Profilhöhe überwiegt die Schubsteifigkeit der Verbindungsmittel um ein Vielfaches $\left(S_{\text {fast }}>>S_{4 \text { _inflex }}\right)$ und führt zu großen Werten $\kappa$. Die Zunahme der Schubsteifigkeit $S_{4 \_ \text {inflex }}$ bei gleichzeitiger Abnahme der Verbindungsmittelsteifigkeit $S_{\text {fast }}$ infolge einer kleineren Anzahl von Verbindungsmittel am Querrand führt zu kleinen Werten $\kappa$ für die schubsteifen Trapezprofile.

In [16] wurde gezeigt, dass die Schubsteifigkeit bei vierseitiger Lagerung unter Berücksichtigung nachgiebiger Verbindungsmittel nur geringfügig von dem Seitenverhältnis $\alpha$ beeinflusst wird (die Schubsteifigkeit wird mit anwachsendem Seitenverhältnis kleiner). Da zudem die üblichen Schubfeldausbildungen Seitenverhältnisse $\alpha<1,0$ besitzen, kann für die nachfolgenden Untersuchungen das in Bild 4.23 untersuchte Seitenverhältnis $\alpha \approx 1,0$ als Grundlage zur Ermittlung der Schubsteifigkeit $\mathrm{S}_{4 \_ \text {flex }}$ herangezogen werden.

Analog zu den Untersuchungen mit vierseitig gelagerten Trapezprofilen wurden solche an zweiseitig gelagerten Trapezprofilen durchgeführt. Hierzu wurden bei dem Finite-Element-Modell lediglich alle Federelemente an den beiden Längsrändern entfernt. Die mit diesem zweiseitig gelagerten Modell unter Berücksichtigung einer Nachgiebigkeit gemäß Bild 4.20 für die Verbindungsmittel am Querrand erhaltenen Schubsteifigkeiten sind in Bild 4.24 in Abhängigkeit vom Profiltyp und vom Seitenverhältnis $\alpha$ dargestellt. 


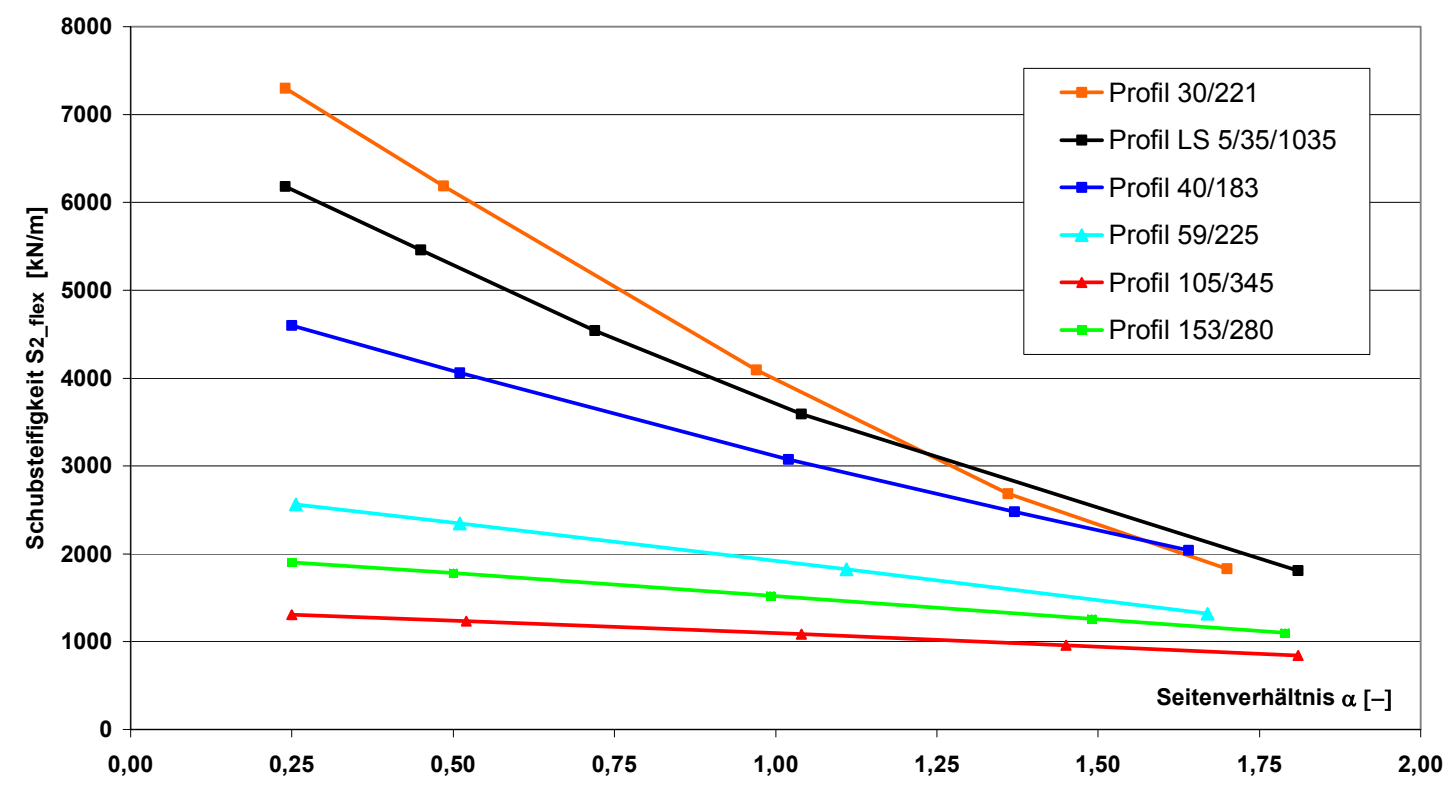

Bild 4.24: Einfluss des Seitenverhältnisses $\alpha$ auf die Schubsteifigkeit bei zweiseitiger Lagerung und Berücksichtigung von nachgiebigen Verbindungsmitteln ( $s_{p}$ gemäß Bild 4.20)

Es ist zu erkennen, dass die Schubsteifigkeit aller Profile mit zunehmendem Seitenverhältnis $\alpha$ kontinuierlich abnimmt, wobei die Abnahme bei den Profilen mit geringer Profilhöhe deutlicher ausgeprägt ist als bei den Profilen mit großer Profilhöhe. Diese Feststellung lässt sich durch Betrachtung eines zweiseitig gelagerten Systems nach Bild 4.25 wie folgt erklären:

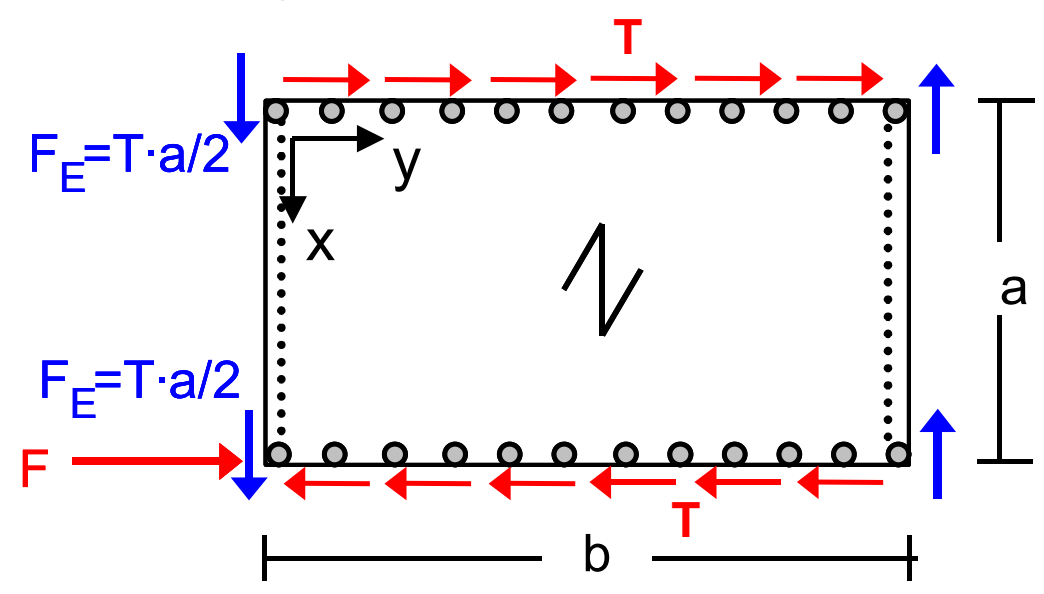

Bild 4.25: System eines zweiseitig gelagerten Schubfeldes

Die äußere Kraft $F$ verteilt sich rechtwinklig zur Profilierung in y-Richtung als näherungsweise konstanter Schubfluss $T$. Die Kraft $F_{E}$, die infolge des Wegfalls der Verbindungsmittel am Längsrand durch die Randrippen auf die Verbindungsmittel am Querrand übertragen werden muss, berechnet sich nach Gleichung (4.10) zu

$$
F_{E}=T \cdot a
$$

und verteilt sich je zur Hälfte auf die beiden Querränder. Diese Kraft ist unabhängig von der Schubfeldbreite und führt zu einem ungleichförmigen Schubspannungszustand im Schubfeld, auf den die deutliche Abnahme der Schubsteifigkeit aus Bild 4.24 mit zunehmendem Seitenverhältnis maßgeblich zurückzuführen ist. 
Die Kraft $F_{E}$ bewirkt neben zusätzlichen Schubverzerrungen und Blechendverwölbungen im Trapezprofil eine erhöhte Belastung der Verbindungsmittel am Querrand, deren Anzahl mit einer verminderten Schubfeldbreite abnimmt. Die aus der Überlagerung der Kräfte in $x$ - und $y$-Richtung resultierenden erhöhten Kräfte der Verbindungsmittel vergrößern die Nachgiebigkeiten und mindern ebenfalls die Steifigkeit des Schubfeldes. Die Zunahme der resultierenden Kräfte im Eckbereich bei steigendem Seitenverhältnis $\alpha$ wird durch Vergleich der numerischen Ergebnisse in Bild 4.26 und Bild 4.27 bestätigt, in denen die auf den nominellen Schubfluss normierten Schraubenkräfte am Querrand für drei Profile dargestellt sind, deren Profilhöhe bei sonst identischen Parametern zwischen $30 \mathrm{~mm}$ und $90 \mathrm{~mm}$ variiert wurde.

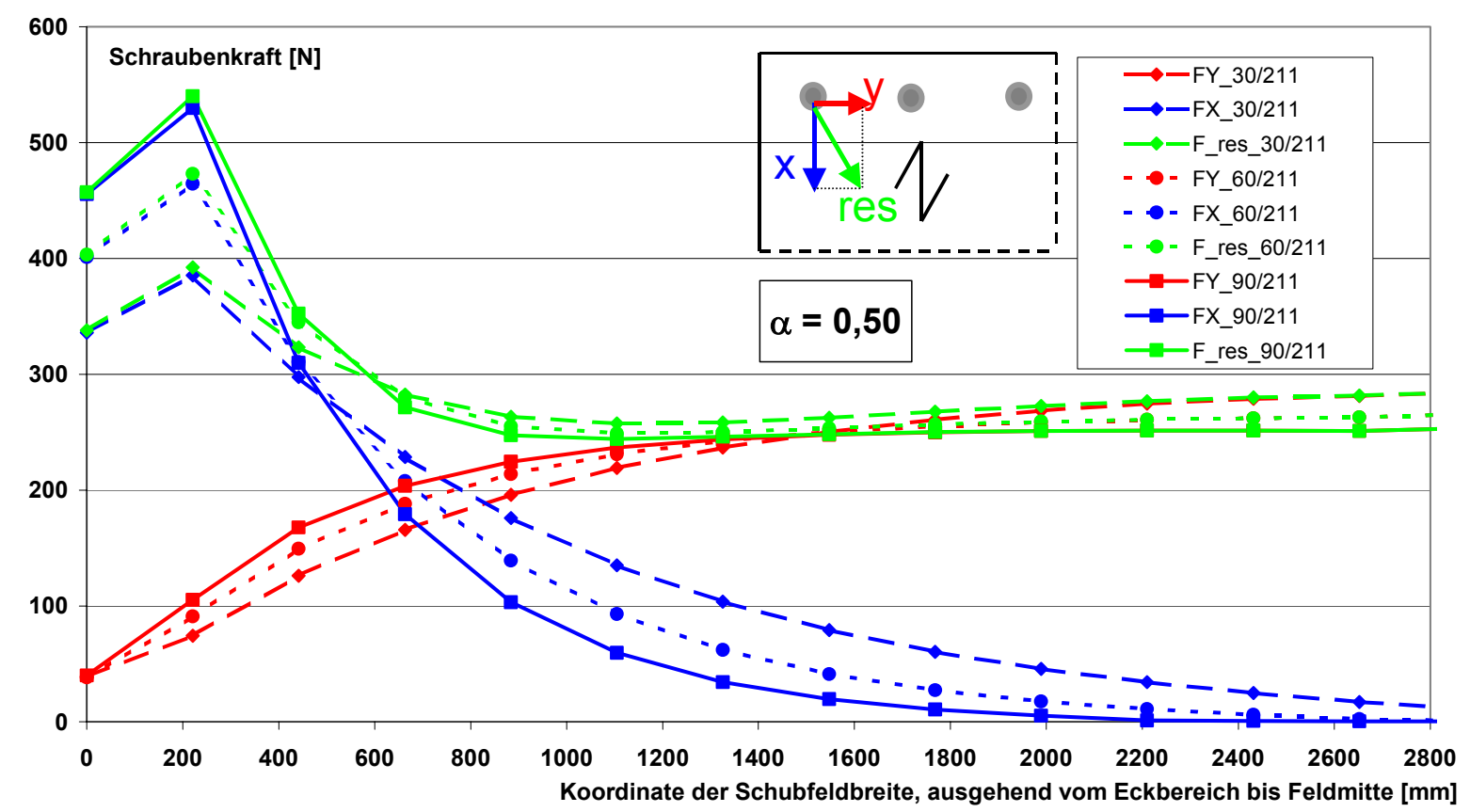

Bild 4.26: Kräfte der Verbindungsmittel am Querrand für $\alpha=0,5$ ( $s_{p}$ gemäß Bild 4.20)

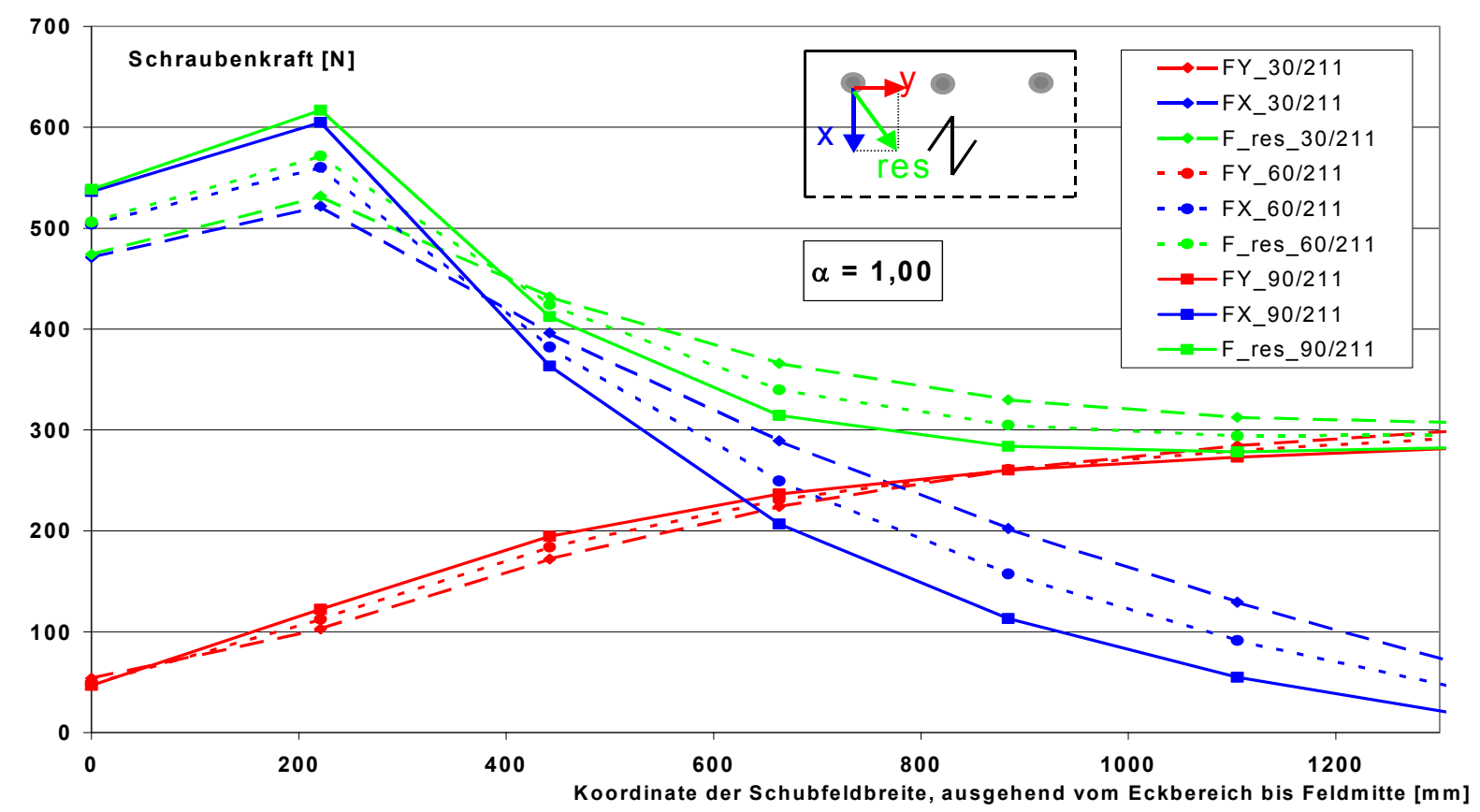

Bild 4.27: Kräfte der Verbindungsmittel am Querrand für $\alpha=1,0$ ( $s_{p}$ gemäß Bild 4.20) 
Beide untersuchten Seitenverhältnisse zeigen, dass die Kräfte in y-Richtung zum Eckbereich hin abfallen und damit kein konstanter Schubfluss in diesem Bereich vorliegt. Während der Verlauf der Schraubenkräfte in y-Richtung für alle drei Profiltypen und beide Seitenverhältnisse nahezu identisch ist, zeigen sich bei den Kräften in xRichtung deutliche Unterschiede. Mit größer werdender Profilhöhe zeigt sich eine Zunahme der Schraubenkräfte im Eckbereich, die im Vergleich zu den schubsteiferen Profilen mit kleiner Profilhöhe in Richtung Feldmitte schneller abklingt. Dadurch entsteht für die hohen, schubweichen Profile eine Lastkonzentration im Eckbereich. Im Gegensatz zu den Annahmen in [52] für ein Schubfeld mit vierseitiger Lagerung liegt damit für den Fall einer zweiseitigen Lagerung kein umlaufend konstanter Schubfluss vor. Lediglich im Bereich der Feldmitte bei kleinen Seitenverhältnissen liegt näherungsweise ein konstanter Schubfluss vor (Bild 4.26). Bei kleinem Seitenverhältnis hat die Randstörung einen geringeren Einfluss auf die Schubsteifigkeit.

In Bild 4.28 ist das Verhältnis der Schubsteifigkeit bei zweiseitiger Lagerung (vgl. Bild 4.24) zu der bei vierseitiger Lagerung für verschiedene Profiltypen über dem Seitenverhältnis $\alpha$ unter Berücksichtigung der Nachgiebigkeit der Verbindungsmittel am Querrand (siehe Bild 4.20) aufgetragen. Dieses Bild zeigt, dass ein Schubfeld mit zweiseitiger Lagerung und unendlicher Breite (Seitenverhältnis $\alpha \rightarrow 0$ ) die gleiche Schubsteifigkeit wie ein umlaufend gelagertes Schubfeld hat und bestätigt, dass die Randstörung mit zunehmender Schubfeldbreite an Einfluss hinsichtlich der Schubsteifigkeit verliert. Die Größe des Steifigkeitsabfalls bei zweiseitiger Lagerung wird maßgeblich durch die Schubsteifigkeit bei vierseitiger Lagerung bestimmt.

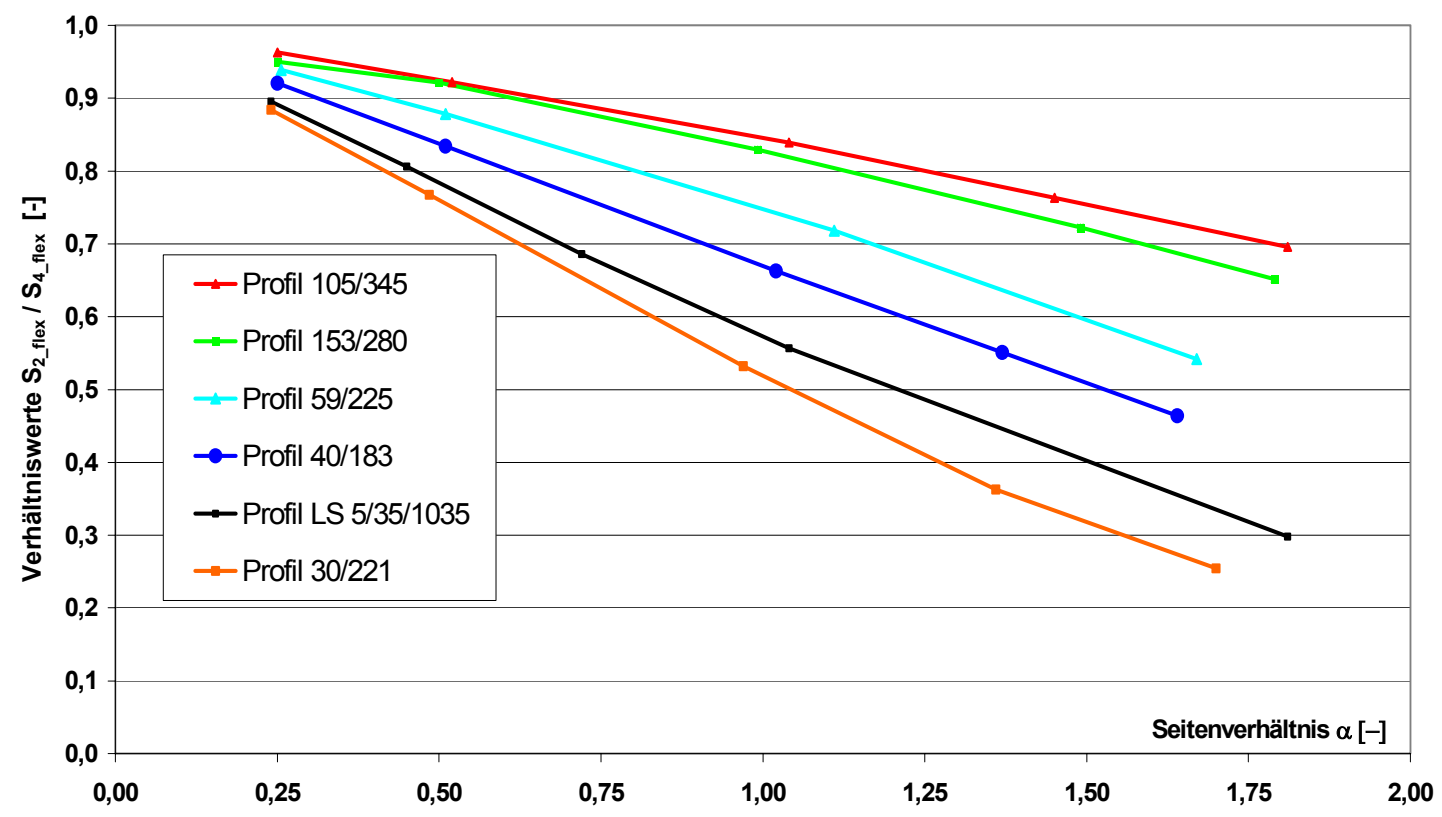

Bild 4.28: Verhältniswerte bei zwei- und vierseitiger Lagerung in Abhängigkeit des Seitenverhältnisses

Entsprechend den Ausführungen aus [16] lassen sich die Funktionen in Bild 4.28 linearisieren und die Schubsteifigkeit $S_{2 \text { flex }}$ bei zweiseitiger Lagerung in Abhängigkeit der Schubsteifigkeit $S_{4}$ flex bei vierseitiger Lagerung in der Form

$$
\mathrm{S}_{2 \_ \text {flex }}=\beta \cdot \mathrm{S}_{4 \_ \text {flex }}
$$

mit 


$$
\beta=1-\gamma \cdot \alpha
$$

darstellen, wobei $\gamma$ der Steigung der linearisierten Funktion entspricht.

Mit den unter Abschnitt 4.1.5 ermittelten Nachgiebigkeiten für unterschiedliche Befestigungsarten am Quer- und Längsrand wurden Finite-Element-Berechnungen unter Berücksichtigung von zwei- und vierseitiger Lagerung durchgeführt. Aus den dabei ermittelten Verhältniswerten $\beta=S_{2 \text { flex }} / S_{4_{f} \text { flex }}$ wurden unter Berücksichtigung des Seitenverhältnisses $\alpha$ die zugehörigen Werte $\gamma$ berechnet. Diese sind für die unterschiedlichen Nachgiebigkeiten in Bild 4.29 in Abhängigkeit der Schubsteifigkeit $\mathrm{S}_{4 \_ \text {flex }}$ dargestellt.

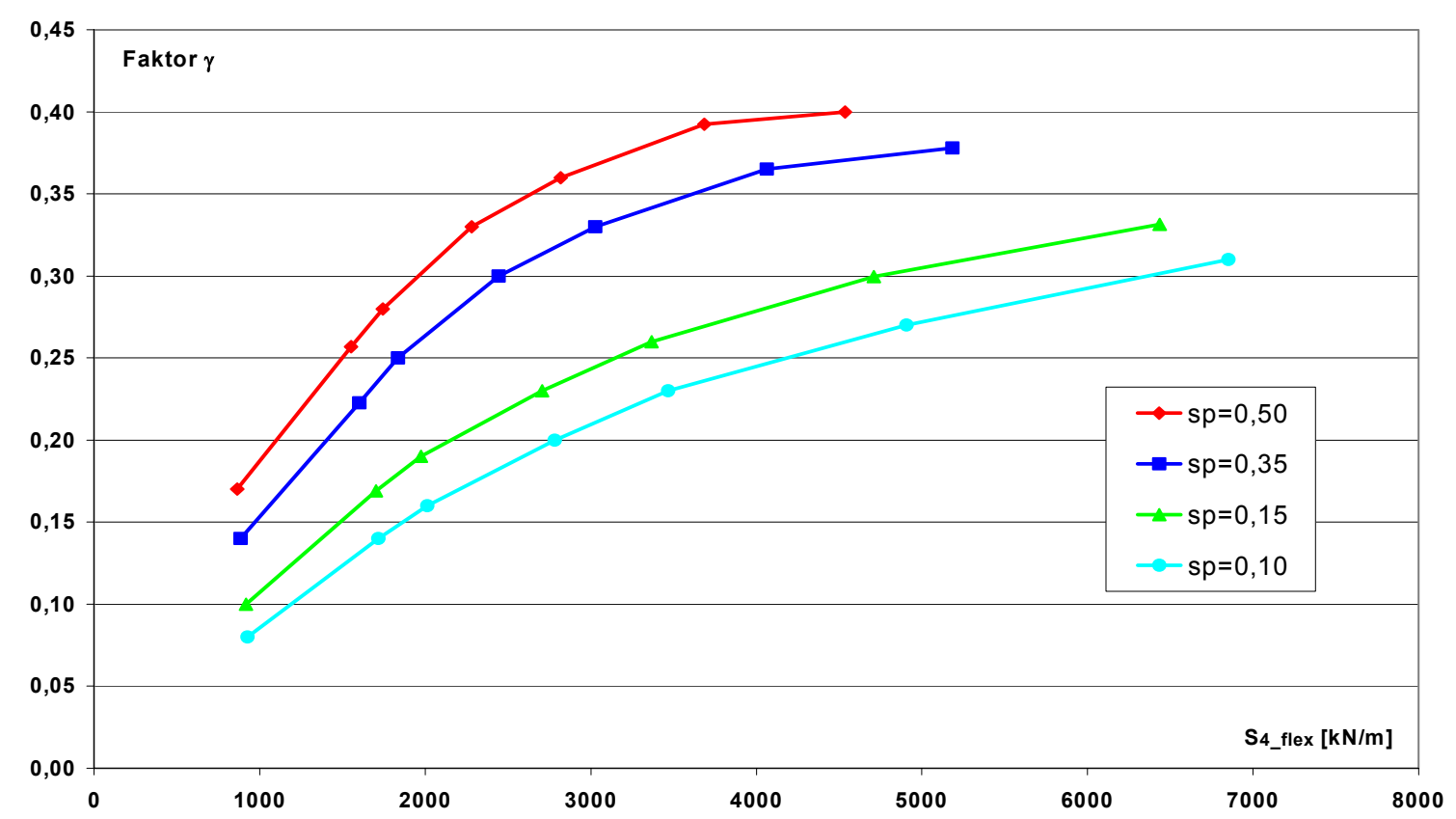

Bild 4.29: Faktoren $\gamma$ für unterschiedliche Nachgiebigkeiten $s_{p}$ der Verbindungsmittel

Mit den voranstehenden Informationen kann die Schubsteifigkeit $S_{2_{-} \text {flex }}$ eines zweiseitig gelagerten Schubfeldes unter Berücksichtigung der in Bild 4.29 aufgeführten Nachgiebigkeiten der Verbindungsmittel am Querrand in Abhängigkeit der Schubsteifigkeit bei unnachgiebiger, vierseitiger Lagerung entsprechend Gleichung (4.13) berechnet werden.

$$
\mathrm{S}_{2_{-} \text {flex }}=(1-\gamma \cdot \alpha) \cdot \kappa \cdot \mathrm{S}_{4_{-} \text {inflex }}
$$

$\begin{array}{lll}\text { mit } & \gamma & \text { aus Bild 4.29 } \\ & \alpha & \text { Seitenverhältnis des Schubfeldes } \\ & \kappa & \text { aus Bild 4.23 } \\ & \mathrm{S}_{4 \_ \text {inflex }} & \text { Schubsteifigkeit nach [53] }\end{array}$




\subsubsection{Einfluss der Nachgiebigkeit der Verbindungsmittel in den Längsstö- ßen}

Die im Abschnitt zuvor aufgeführten Untersuchungsergebnisse gehen von Schubfeldern aus, deren einzelne Profiltafeln unnachgiebig in den Längsstößen miteinander verbunden sind. Im Fall einer vierseitigen Lagerung liegt ein umlaufend konstanter Schubfluss vor. Die daraus resultierende, zusätzlich auftretende Schubfeldverformung aufgrund von Nachgiebigkeiten in den Längsstoßverbindungen ist vernachlässigbar klein. Im Gegensatz dazu entstehen bei zweiseitiger Schubfeldlagerung aufgrund der fehlenden Längsrandbefestigung erheblich größere Beanspruchungen parallel zur Profilierung (vgl. Bild 4.26und Bild 4.27), die zusätzliche Nachgiebigkeiten in den Längsstößen bewirken und die Schubfeldsteifigkeit mindern.

Die Anzahl der Längsstöße pro Schubfeld ist $\mathrm{n}_{\mathrm{sh}}-1$, wenn $\mathrm{n}_{\text {sh }}$ die Anzahl der Profiltafeln ist. Nach DIN 18807 Teil 3 und DIN EN 1993-1-3 können die Längsabstände $e_{L}$ der Längsstoßverbinder zwischen $50 \mathrm{~mm}$ und $666 \mathrm{~mm}$ variieren. Die Art der Längsstoßverbindung bestimmt deren Nachgiebigkeit $\mathrm{s}_{\mathrm{s}}$. Entsprechend Abschnitt 4.2.2 werden die Längsstoßverbindungen (Schrauben und Niete) vereinfacht mit einer Nachgiebigkeit $\mathrm{s}_{\mathrm{s}}=0,30 \mathrm{~mm} / \mathrm{kN}$ angesetzt.

Aufgrund der Vielzahl der aufgeführten Parameter ist es zweckmäßig, ein analytisches Verfahren zur Bestimmung des Einflusses der Längsstoßverbindungen heranzuziehen und dieses durch begleitende numerische Berechnungen zu verifizieren. Hierzu wird aus dem System eines zweiseitig gelagerten Schubfeldes entsprechend Bild 4.25 der Querrand betrachtet, und an diesem werden die Schraubenkräfte in Rippenlängsrichtung als Funktion dritter Ordnung angesetzt. Im Gegensatz zu [5], wo die Annahme einer linearen Verteilung getroffen wurde, bildet die Näherungsfunktion dritter Ordnung den Verlauf der Schraubenkräfte im Bezug auf Bild 4.26 wesentlich besser ab. Es ergibt sich das in Bild 4.30 dargestellte System:

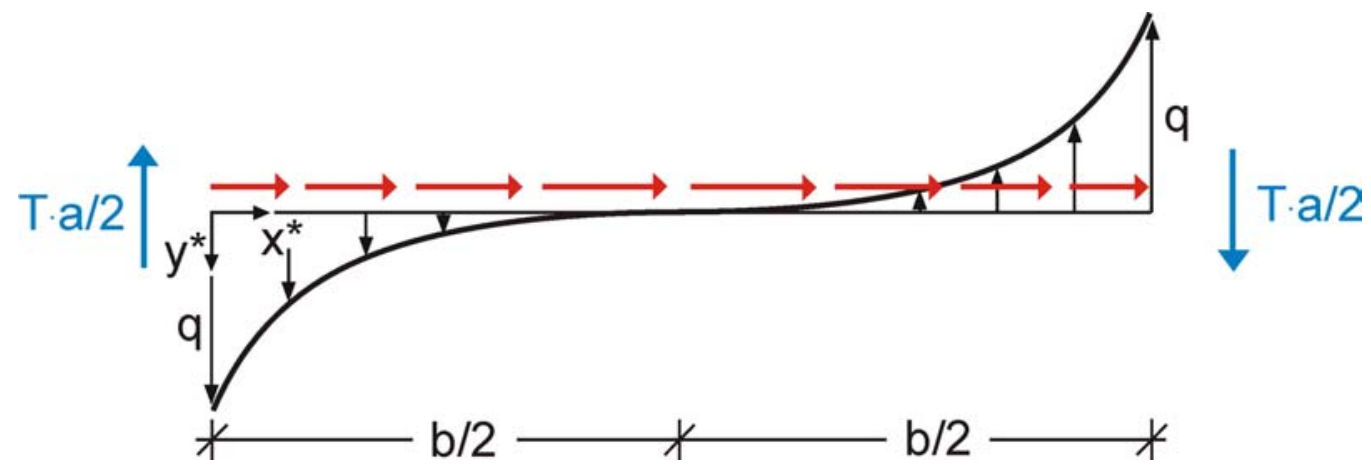

Bild 4.30: Kräfte am System des Querrands

Das Momentengleichgewicht am System liefert

$$
q=5 \cdot \frac{T \cdot a}{b}
$$

Der Verlauf der Schubkräfte im Trapezprofil kann in Rippenlängsrichtung unter Berücksichtigung beider Querränder durch die Funktion

$$
y^{*}=q \cdot \frac{8}{b^{3}} \cdot\left(\frac{b}{2}-x^{*}\right)^{3} \cdot 2
$$

dargestellt werden. 
Die Integration des Schubkraftverlaufs ergibt für den i-ten Längsstoß mit

$$
x^{*}=i \cdot \frac{b}{n_{s h}}
$$

eine Schubkraft von

$$
\mathrm{Q}=40 \cdot \mathrm{T} \cdot \mathrm{a} \cdot\left[\frac{\mathrm{i}}{4 \cdot \mathrm{n}_{\mathrm{sh}}}-\frac{3 \cdot \mathrm{i}^{2}}{4 \cdot \mathrm{n}_{\mathrm{sh}}{ }^{2}}+\frac{\mathrm{i}^{3}}{\mathrm{n}_{\mathrm{sh}}{ }^{3}}-\frac{\mathrm{i}^{4}}{2 \cdot \mathrm{n}_{\mathrm{sh}}{ }^{4}}\right]
$$

Die Schubverformung aus der Nachgiebigkeit der Verbindungen im i-ten Längsstoß berechnet sich zu

$$
\Delta \mathrm{v}_{\mathrm{Ss}}=40 \cdot \mathrm{T} \cdot \mathrm{a} \cdot\left[\frac{\mathrm{i}}{4 \cdot \mathrm{n}_{\mathrm{sh}}}-\frac{3 \cdot \mathrm{i}^{2}}{4 \cdot \mathrm{n}_{\mathrm{sh}}{ }^{2}}+\frac{\mathrm{i}^{3}}{\mathrm{n}_{\mathrm{sh}}{ }^{3}}-\frac{\mathrm{i}^{4}}{2 \cdot \mathrm{n}_{\mathrm{sh}}{ }^{4}}\right] \cdot \frac{\mathrm{s}_{\mathrm{s}}}{\mathrm{n}_{\mathrm{s}}}
$$

Die Gesamtschubverformung $\Delta \mathrm{v}_{\mathrm{Ss}}$ aus der Nachgiebigkeit aller Längsstöße wird durch Gleichung (4.19) wiedergegeben:

$$
\Delta \mathrm{v}_{\mathrm{Ss}}=40 \cdot \mathrm{T} \cdot \mathrm{a} \cdot \sum_{\mathrm{i}=1}^{\mathrm{i}=\left(\mathrm{n}_{\mathrm{sh}}-1\right)}\left[\frac{\mathrm{i}}{4 \cdot \mathrm{n}_{\mathrm{sh}}}-\frac{3 \cdot \mathrm{i}^{2}}{4 \cdot \mathrm{n}_{\mathrm{sh}}{ }^{2}}+\frac{\mathrm{i}^{3}}{\mathrm{n}_{\mathrm{sh}}{ }^{3}}-\frac{\mathrm{i}^{4}}{2 \cdot \mathrm{n}_{\mathrm{sh}}{ }^{4}}\right] \cdot \frac{\mathrm{s}_{\mathrm{s}}}{\mathrm{n}_{\mathrm{s}}}
$$

Die Auswertung des Summenausdrucks aus Gleichung (4.19) ist multipliziert mit dem Faktor 40 in Bild 4.31 in Abhängigkeit der Profiltafelanzahl $\mathrm{n}_{\mathrm{sh}}$ dargestellt.

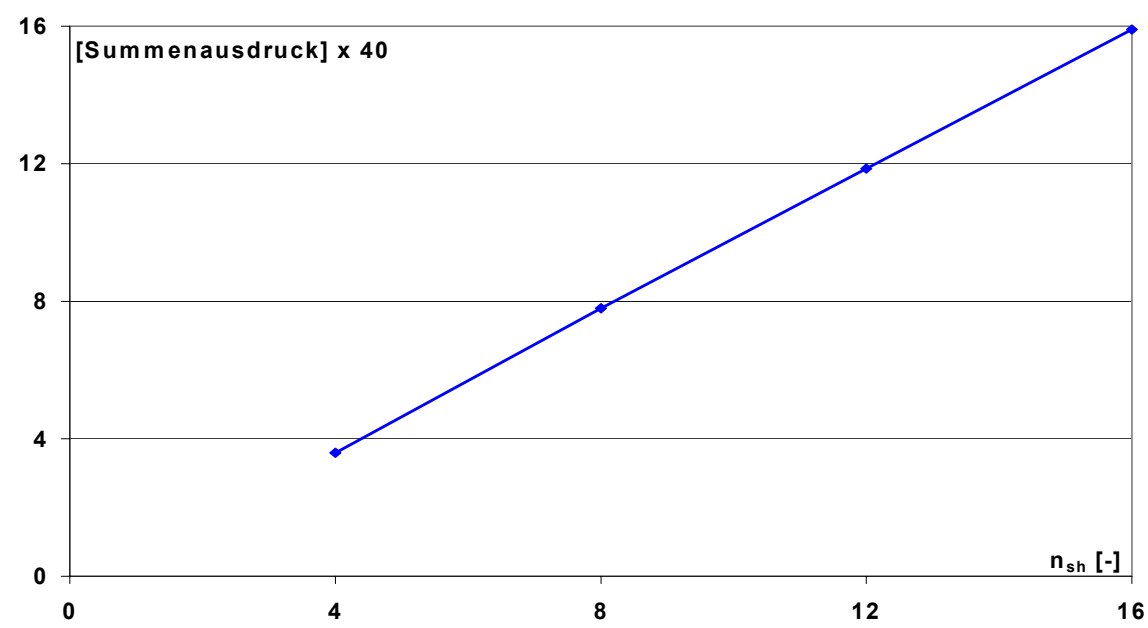

Bild 4.31: grafische Auswertung des Summenausdrucks aus Gleichung (4.19)

Daraus ist zu erkennen, dass die Gesamtschubverformung $\Delta v_{S s}$ aus der Nachgiebigkeit der Verbindungen aller Längsstöße ausreichend genau durch Gleichung (4.20)

$$
\Delta v_{S s}=n_{s h} \cdot T \cdot a \cdot \frac{s_{s}}{n_{s}}
$$

angenähert werden kann. 


\subsection{Erstellung eines Rechenmodells}

Auf Grundlage der Untersuchungen aus den Abschnitten 4.2.5.3 und 4.2.5.4 lässt sich ein Rechenmodell herleiten, mit dem die Ermittlung der Schubsteifigkeit bei zweiseitiger Lagerung unter Berücksichtigung der Nachgiebigkeiten aller Verbindungsmittel möglich ist.

Entsprechend Gleichung (4.2) ist die Schubsteifigkeit linear von der Gesamtschubfeldverformung abhängig. Deswegen können die Verformungen aus der Nachgiebigkeit der Verbindungsmittel am Querrand und den Längsstößen mit den Verformungsanteilen aus der Schubverzerrung und der Blechendverwölbung nach [52] überlagert werden. Analog zu den Ausführungen aus Abschnitt 4.2.5.3 wird zur Berechnung der Schubsteifigkeit $S_{2}$ unter Berücksichtigung der Nachgiebigkeit aller Verbindungsmittel folgendes Federgesetz angewandt:

$$
\frac{1}{\mathrm{~S}_{2}}=\frac{1}{\mathrm{~S}_{2 \_ \text {flex }}}+\frac{1}{\mathrm{~S}_{2 \_\mathrm{ss}}}
$$

mit $\quad S_{2}$

$$
\begin{array}{ll}
\mathrm{S}_{2} \text { flex } & \text { Schubsteifigkeit gemäß Gleichung (4.13) } \\
\mathrm{S}_{2 \_s s} & \text { Schubsteifigkeit der Längsstoßverbindungen }
\end{array}
$$

Aus Gleichung (4.21) folgt die Darstellung für die Schubsteifigkeit $S_{2} z u$

$$
\mathrm{S}_{2}=\frac{\mathrm{S}_{2 \_ \text {flex }} \cdot \mathrm{S}_{2 \_\mathrm{ss}}}{\mathrm{S}_{2_{-} \text {flex }}+\mathrm{S}_{2 \_\mathrm{ss}}}=\mathrm{S}_{2_{-} \mathrm{flex}} \cdot\left(1+\frac{\mathrm{S}_{2 \_ \text {flex }}}{\mathrm{S}_{2 \_\mathrm{ss}}}\right)^{-1}
$$

Der Klammerausdruck in Gleichung (4.22) kann über den Verhältniswert $\eta$ entsprechend Gleichung (4.23)

$$
\eta=\frac{\mathrm{S}_{2}}{\mathrm{~S}_{2_{-} \text {flex }}}=\left(1+\frac{\alpha \cdot \Delta \mathrm{v}_{\mathrm{ss}}}{\Delta \mathrm{v}_{\mathrm{sp}}}\right)^{-1}=\frac{\Delta \mathrm{v}_{\mathrm{Sp}}}{\Delta \mathrm{v}_{\mathrm{Sp}}+\alpha \cdot \Delta \mathrm{v}_{\mathrm{Ss}}}
$$

dargestellt werden, wobei

$\Delta \mathrm{v}_{\mathrm{Sp}}$ die der Schubsteifigkeit $\mathrm{S}_{2}$ flex zugrunde liegende Schubfeldverformung ist und durch den Faktor $\alpha$ berücksichtigt wird, dass die Nachgiebigkeit der Längsstoßverbindungen mit abnehmendem Seitenverhältnis $\alpha$ an Einfluss verliert.

Unter Berücksichtigung von Gleichung (4.2) lässt sich $\Delta \mathrm{v}_{\mathrm{Sp}}$ in Abhängigkeit von $\mathrm{S}_{2 \text { flex }}$ darstellen. Dadurch ergibt sich aus Gleichung (4.23) die nachfolgende Darstellung für den Abminderungsfaktor $\eta$

$$
\eta=\frac{1}{1+\alpha \cdot \mathrm{n}_{\mathrm{sh}} \cdot \frac{\mathrm{s}_{\mathrm{s}}}{\mathrm{n}_{\mathrm{s}}} \cdot \mathrm{S}_{2_{-} \text {flex }}}
$$

und zur Berechnung der Schubsteifigkeit $S_{2}$ die Gleichung (4.25) 


$$
\mathrm{S}_{2}=\frac{\mathrm{S}_{2 \_ \text {flex }}}{1+\alpha \cdot \mathrm{n}_{\mathrm{sh}} \cdot \frac{\mathrm{s}_{\mathrm{s}}}{\mathrm{n}_{\mathrm{s}}} \cdot \mathrm{S}_{2 \_ \text {flex }}}
$$

mit $\quad S_{2}$ flex $\quad$ aus Gleichung (4.13)

$\alpha \quad$ Seitenverhältnis des Schubfeldes gemäß Gleichung (4.6)

$\mathrm{n}_{\mathrm{sh}} \quad$ Anzahl der Profiltafeln

$\mathrm{s}_{\mathrm{s}} \quad$ Nachgiebigkeit der Längsstoßverbindung

$\mathrm{n}_{\mathrm{s}} \quad$ Anzahl der Verbindungsmittel pro Längsstoß

$\mathrm{e}_{\mathrm{L}} \quad$ Abstand der Verbindungsmittel im Längsstoß $\leq 500 \mathrm{~mm}$

Zur Beurteilung der Güte des Rechenmodells sind in Tabelle 4.9 und Tabelle 4.10 die durch Finite-Element-Berechnung entsprechend Abschnitt 4.2 ermittelten Schubsteifigkeiten verschiedener Trapezprofile den rechnerisch ermittelten Ergebnissen gegenübergestellt. Der Vergleich der Schubsteifigkeiten erfolgt sowohl in Hinblick auf die Schubsteifigkeit $S_{2 \text { flex }}$ (ohne Berücksichtigung der Längsstöße) als auch hinsichtlich der Schubsteifigkeit $\mathrm{S}_{2}$ (mit Berücksichtigung der Längsstöße). Zur Abdeckung eines möglichst großen Parameterbereichs erfolgt der Vergleich für drei Trapezprofile mit Profilhöhen zwischen $35 \mathrm{~mm}$ und $153 \mathrm{~mm}$, Schubfeldlängen $\mathrm{I}_{\mathrm{s}} \mathrm{zwi}-$ schen $3000 \mathrm{~mm}$ und $5000 \mathrm{~mm}$, Längsstoßabständen $\mathrm{e}_{\mathrm{L}}$ zwischen $50 \mathrm{~mm}$ und $500 \mathrm{~mm}$ und Nachgiebigkeiten $\mathrm{s}_{\mathrm{p}}$ der Verbindungsmittel am Querrand von $0,10 \mathrm{~mm} / \mathrm{kN}$ und $0,35 \mathrm{~mm} / \mathrm{kN}$. 


\begin{tabular}{|c|c|c|c|c|c|c|c|c|c|}
\hline \multirow{3}{*}{ Profil } & \multirow{3}{*}{$\mathrm{I}_{\mathrm{s}}[\mathrm{mm}]$} & \multirow{3}{*}{$\alpha[-]$} & \multirow{3}{*}{$\mathrm{e}_{\mathrm{L}}[\mathrm{mm}]$} & \multicolumn{4}{|c|}{ Schubsteifigkeiten [kN/m] } & \multirow{2}{*}{\multicolumn{2}{|c|}{ Abweichungen [\%] }} \\
\hline & & & & \multicolumn{2}{|c|}{ nach FE-Berechnung } & \multicolumn{2}{|c|}{ nach Rechenmodell } & & \\
\hline & & & & $\mathrm{S}_{2 \text { flex }}$ & $\mathrm{S}_{2}$ & $\mathrm{~S}_{2 \text { flex }}$ & $\mathrm{S}_{2}$ & $\mathrm{~S}_{2 \text { flex }}$ & $\mathrm{S}_{2}$ \\
\hline \multirow{18}{*}{$35 / 207$} & \multirow{18}{*}{3000} & \multirow{6}{*}{0,26} & ohne & 7005 & - & 6074 & - & -13 & - \\
\hline & & & 50 & - & 6279 & - & 5575 & & -11 \\
\hline & & & 100 & - & 5450 & - & 5138 & & -6 \\
\hline & & & 200 & - & 4678 & - & 4410 & & -6 \\
\hline & & & 300 & - & 4257 & - & 3828 & & -10 \\
\hline & & & 500 & - & 3621 & - & 2954 & & -18 \\
\hline & & \multirow{6}{*}{0,58} & ohne & 6047 & - & 5419 & - & -10 & - \\
\hline & & & 50 & - & 5469 & - & 5018 & - & -8 \\
\hline & & & 100 & - & 4819 & - & 4662 & - & -3 \\
\hline & & & 200 & - & 4202 & - & 4054 & - & -4 \\
\hline & & & 300 & - & 3861 & - & 3557 & - & -8 \\
\hline & & & 500 & - & 3338 & - & 2790 & - & -16 \\
\hline & & \multirow{6}{*}{0,97} & ohne & 4918 & - & 4620 & - & -6 & - \\
\hline & & & 50 & - & 4519 & - & 4325 & - & -4 \\
\hline & & & 100 & - & 4073 & - & 4058 & - & 0 \\
\hline & & & 200 & - & 3637 & - & 3590 & - & -1 \\
\hline & & & 300 & - & 3388 & - & 3194 & - & -6 \\
\hline & & & 500 & - & 2998 & - & 2562 & - & -15 \\
\hline \multirow{36}{*}{$59 / 225$} & \multirow{18}{*}{3000} & \multirow{6}{*}{0,37} & ohne & 2657 & - & 2614 & - & -2 & - \\
\hline & & & 50 & - & 2502 & - & 2503 & - & 0 \\
\hline & & & 100 & - & 2282 & - & 2398 & - & 5 \\
\hline & & & 200 & - & 2038 & - & 2203 & - & 8 \\
\hline & & & 300 & - & 1909 & - & 2026 & - & 6 \\
\hline & & & 500 & - & 1731 & - & 1717 & - & -1 \\
\hline & & & ohne & 2452 & - & 2444 & - & 0 & - \\
\hline & & & 50 & - & 2318 & - & 2346 & - & 1 \\
\hline & & & 100 & - & 2128 & - & 2253 & - & 6 \\
\hline & & $0,6 /$ & 200 & - & 1918 & - & 2079 & - & 8 \\
\hline & & & 300 & - & 1805 & - & 1920 & - & 6 \\
\hline & & & 500 & - & 1650 & - & 1639 & - & -1 \\
\hline & & & ohne & 2147 & - & 2196 & - & 2 & - \\
\hline & & & 50 & - & 2044 & - & 2117 & - & 4 \\
\hline & & 111 & 100 & - & 1900 & - & 2042 & - & 7 \\
\hline & & 1,11 & 200 & - & 1739 & - & 1899 & - & 9 \\
\hline & & & 300 & - & 1651 & - & 1766 & - & 7 \\
\hline & & & 500 & - & 1529 & - & 1526 & - & 0 \\
\hline & & & ohne & 4242 & - & 3927 & - & -7 & - \\
\hline & & & 50 & - & 3893 & - & 3684 & - & -5 \\
\hline & & 037 & 100 & - & 3442 & - & 3465 & - & 1 \\
\hline & & I I & 200 & - & 2964 & - & 3086 & - & 4 \\
\hline & & & 300 & - & 2676 & - & 2770 & - & 4 \\
\hline & & & 500 & - & 2278 & - & 2275 & - & 0 \\
\hline & & & ohne & 3945 & - & 3645 & - & -8 & - \\
\hline & & & 50 & - & 3630 & - & 3433 & - & -5 \\
\hline & 5000 & ח9? & 100 & - & 3225 & - & 3241 & - & 0 \\
\hline & 3000 & 0,02 & 200 & - & 2795 & - & 2906 & - & 4 \\
\hline & & & 300 & - & 2535 & - & 2627 & - & 4 \\
\hline & & & 500 & - & 2172 & - & 2172 & - & 0 \\
\hline & & & ohne & 3354 & - & 3091 & - & -8 & - \\
\hline & & & 50 & - & 3102 & - & 2938 & - & -5 \\
\hline & & 111 & 100 & - & 2795 & - & 2797 & - & 0 \\
\hline & & 1,11 & 200 & - & 2461 & - & 2545 & - & 3 \\
\hline & & & 300 & - & 2254 & - & 2326 & - & 3 \\
\hline & & & 500 & - & 1961 & - & 1966 & - & 0 \\
\hline
\end{tabular}

Tabelle 4.9: Vergleich der Steifigkeiten aus FE-Berechnung und Rechenmodell $\left(s_{p}=0,10 \mathrm{~mm} / \mathrm{kN}\right)$ 


\begin{tabular}{|c|c|c|c|c|c|c|c|c|c|c|}
\hline \multirow{3}{*}{ Profil } & \multirow{3}{*}{$\begin{array}{c}\mathrm{I}_{\mathrm{s}} \\
{[\mathrm{mm}]}\end{array}$} & \multirow{3}{*}{$\alpha[-]$} & \multirow{3}{*}{$\begin{array}{c}\mathrm{s}_{\mathrm{p}} \\
{[\mathrm{mm} / \mathrm{kN}]}\end{array}$} & \multirow{3}{*}{$e_{L}[\mathrm{~mm}]$} & \multicolumn{4}{|c|}{ Schubsteifigkeiten $[\mathrm{kN} / \mathrm{m}]$} & \multirow{2}{*}{\multicolumn{2}{|c|}{$\begin{array}{c}\text { Abweichungen } \\
{[\%]}\end{array}$}} \\
\hline & & & & & \multicolumn{2}{|c|}{ nach FE-Berechnung } & \multicolumn{2}{|c|}{ nach Rechenmodell } & & \\
\hline & & & & & $\mathrm{S}_{2 \text { flex }}$ & $\mathrm{S}_{2}$ & $S_{2 \text { flex }}$ & $\mathrm{S}_{2}$ & $\mathrm{~S}_{2 \text { flex }}$ & $\mathrm{S}_{2}$ \\
\hline \multirow{30}{*}{$153 / 280$} & \multirow{30}{*}{5000} & \multirow{10}{*}{0,26} & 0,10 & ohne & 1915 & - & 1787 & - & -7 & - \\
\hline & & & 0,10 & 50 & - & 1733 & - & 1731 & - & 0 \\
\hline & & & 0,10 & 100 & - & 1602 & - & 1678 & - & 5 \\
\hline & & & 0,10 & 250 & - & 1335 & - & 1530 & - & 15 \\
\hline & & & 0,10 & 500 & - & 1109 & - & 1319 & - & 19 \\
\hline & & & 0,35 & ohne & 1698 & - & 1553 & - & -9 & - \\
\hline & & & 0,35 & 50 & - & 1606 & - & 1590 & - & -1 \\
\hline & & & 0,35 & 100 & - & 1487 & - & 1545 & - & 4 \\
\hline & & & 0,35 & 250 & - & 1243 & - & 1419 & - & 14 \\
\hline & & & 0,35 & 500 & - & 1034 & - & 1236 & - & 20 \\
\hline & & \multirow{10}{*}{0,85} & 0,10 & ohne & 1669 & - & 1623 & - & -3 & - \\
\hline & & & 0,10 & 50 & - & 1518 & - & 1577 & - & 4 \\
\hline & & & 0,10 & 100 & - & 1415 & - & 1532 & - & 8 \\
\hline & & & 0,10 & 250 & - & 1200 & - & 1408 & - & 17 \\
\hline & & & 0,10 & 500 & - & 1014 & - & 1228 & - & 21 \\
\hline & & & 0,35 & ohne & 1443 & - & 1390 & - & -4 & - \\
\hline & & & 0,35 & 50 & - & 1321 & - & 1356 & - & 3 \\
\hline & & & 0,35 & 100 & - & 1235 & - & 1323 & - & 7 \\
\hline & & & 0,35 & 250 & - & 1058 & - & 1229 & - & 16 \\
\hline & & & 0,35 & 500 & - & 896 & - & 1090 & - & 22 \\
\hline & & \multirow{10}{*}{1,98} & 0,10 & ohne & 1227 & - & 1308 & - & 7 & - \\
\hline & & & 0,10 & 50 & - & 1139 & - & 1278 & - & 12 \\
\hline & & & 0,10 & 100 & - & 1079 & - & 1248 & - & 16 \\
\hline & & & 0,10 & 250 & - & 954 & - & 1165 & - & 22 \\
\hline & & & 0,10 & 500 & - & 834 & - & 1038 & - & 24 \\
\hline & & & 0,35 & ohne & 912 & - & 916 & - & 0 & - \\
\hline & & & 0,35 & 50 & - & 859 & - & 901 & - & 5 \\
\hline & & & 0,35 & 100 & - & 823 & - & 886 & - & 8 \\
\hline & & & 0,35 & 250 & - & 744 & - & 843 & - & 13 \\
\hline & & & 0,35 & 500 & - & 665 & - & 775 & - & 17 \\
\hline
\end{tabular}

Tabelle 4.10: Vergleich der Steifigkeiten aus FE-Berechnung und Rechenmodell

Der Vergleich der in obigen Tabellen aufgeführten Schubsteifigkeiten $S_{2 \text { flex }}$ zeigt bei allen untersuchten Profilen eine gute Übereinstimmung zwischen den Werten nach der Finite-Element-Berechnung und denen des Rechenmodells. Die Abweichungen liegen in einem sehr engen Streubereich. Die maximale Abweichung beträgt $13 \%$, wobei in diesem Fall die mit dem Rechenmodell ermittelte Schubsteifigkeit die Steifigkeit nach der Finite-Element-Berechnung unterschätzt.

Hinsichtlich der Schubsteifigkeit $S_{2}$ zeigte sich beim Profil 59/225 für beide untersuchten Schubfeldlängen eine gute Übereinstimmung der Werte nach dem Rechenmodell und der Finite-Element-Berechnung. Beim Profil 35/207 wurde eine maximale Abweichung von 18\% festgestellt. Bei diesem Profil traten die maximalen Abweichungen in allen Fällen in Verbindung mit Verbindungsmittelabständen $\mathrm{e}_{\mathrm{L}}=500 \mathrm{~mm}$ auf, wobei die Steifigkeit nach dem Rechenmodell kleiner ist als die nach der Finite-Element-Berechnung. Die mit dem Rechenmodell für das Profil 153/280 ermittelten Schubsteifigkeiten überschätzen die Werte nach der FiniteElement-Berechnung um etwa 20 \% für den Fall von Verbindungsmittelabständen $\mathrm{e}_{\mathrm{L}} \geq 250 \mathrm{~mm}$. Diese relativ großen Abweichungen stellen jedoch eine Ausnahme dar und treten nur für den genannten Fall auf. Bei Verbindungsmittelabständen $\mathrm{e}_{\mathrm{L}}<250 \mathrm{~mm}$ sind diese Abweichungen deutlich geringer. Im allgemeinen lässt sich feststellen, dass das Rechenmodell die Werte nach der Finite-Element-Berechnung mit einer ausreichenden Genauigkeit wiedergibt. 
Eine weitere Möglichkeit zur Beurteilung der Güte des Rechenmodells bieten die in [38] aufgeführten Finite-Element-Berechnungen an zwei unterschiedlichen Trapezprofilen.

Dazu werden die in Tabelle 4.11 aufgeführten Einzelfälle mit den Ergebnissen des zuvor entwickelten Rechenmodells verglichen. Da die in [38] ermittelten Schubsteifigkeiten für zweiseitige Lagerung im Verhältnis zur Schubsteifigkeit bei vierseitiger Lagerung angegeben werden, erfolgt der Vergleich mit dem Rechenmodell anhand der berechneten Werte $\mathrm{S}_{2} / \mathrm{S}_{4}$ flex. Ein Vergleich der Einzelwerte der Steifigkeiten ist jedoch nicht möglich, weil diese in [38] nicht angegeben werden.

\begin{tabular}{|c|c|c|c|c|c|c|c|c|c|}
\hline \multicolumn{4}{|c|}{ Eingangswerte } & \multicolumn{4}{|c|}{ Werte nach Rechenmodell } & \multirow{2}{*}{$\begin{array}{c}\mathrm{S}_{2} / \mathrm{S}_{4} \\
\text { nach [38] }\end{array}$} & \multirow{2}{*}{$\begin{array}{l}\text { Abwei- } \\
\text { chung }\end{array}$} \\
\hline \multirow{2}{*}{ Profiltyp } & $\mathrm{I}_{\mathrm{s}}$ & $\mathrm{S}_{4 \text { _inflex }}$ nach [53] & $\alpha$ & $\mathrm{S}_{4_{\text {fflex }}}$ & $\mathrm{S}_{2 \text { flex }}$ & $\mathrm{S}_{2}$ & $\mathrm{~S}_{2} / \mathrm{S}_{4_{\text {fflex }}}$ & & \\
\hline & {$[\mathrm{m}]$} & {$[\mathrm{kN} / \mathrm{m}]$} & {$[-]$} & {$[\mathrm{kN} / \mathrm{m}]$} & {$[\mathrm{kN} / \mathrm{m}]$} & {$[\mathrm{kN} / \mathrm{m}]$} & {$[\mathrm{kN} / \mathrm{m}]$} & {$[-]$} & [\%] \\
\hline \multirow{3}{*}{$39 / 183 / 1,25$} & \multirow{3}{*}{2,0} & \multirow{3}{*}{7250} & 0,36 & 4640 & 4021 & 3358 & 0,72 & 0,75 & 4 \\
\hline & & & 0,27 & 4640 & 4176 & 3466 & 0,75 & 0,79 & 5 \\
\hline & & & 0,20 & 4640 & 4297 & 3537 & 0,76 & 0,84 & 11 \\
\hline \multirow{3}{*}{$200 / 420 / 1,0$} & \multirow{3}{*}{6,0} & \multirow{3}{*}{638} & 1,19 & 593 & 494 & 480 & 0,81 & 0,75 & -7 \\
\hline & & & 0,79 & 593 & 527 & 512 & 0,86 & 0,80 & -7 \\
\hline & & & 0,60 & 593 & 543 & 526 & 0,89 & 0,84 & -6 \\
\hline
\end{tabular}

Tabelle 4.11: Vergleich der Steifigkeiten aus FE-Berechnung nach [38] und Rechenmodell

Die Geringfügigkeit der Abweichungen zeigt, dass das Rechenmodell die Werte der Finite-Element-Berechnung nach [38] gut annähert.

Zusammenfassend kann mit den zuvor aufgeführten Ergebnissen (Tabelle 4.9 Tabelle 4.11) festgestellt werden, dass das entwickelte Rechenmodell für die näherungsweise Berechnung der Schubsteifigkeit eines zweiseitig gelagerten Schubfeldes unter Berücksichtigung der Nachgiebigkeiten in den Längsstößen und am Querrand gut geeignet ist.

\section{Zusammenfassung und Ausblick}

\subsection{Zusammenfassung}

In dieser Arbeit wurde der stabilisierende Einfluss von Trapezprofilen und Sandwichelementen beim Biegedrillknicken stabilitätsgefährdeter Träger untersucht. Dies betrifft zum einen im Hinblick auf die Einschränkung der seitlichen Ausweichmöglichkeit die Schubsteifigkeit von zweiseitig gelagerten Stahltrapezprofilen, zum anderen die drehbettende Wirkung von Sandwichelementen zur Behinderung der Verdrehung. Für beide Stabilisierungsarten wurden mit Hilfe von experimentellen Untersuchungen und Finite-Element-Berechnungen Berechnungsvorschriften entwickelt.

Parametrische Untersuchungen mit einem verifizierten Finite-Element-Modell und eine statistische Auswertung experimenteller Ergebnisse ergaben, dass die drehstabilisierende Wirkung von Sandwichelementen mit Kernschichten aus PolyurethanHartschaum und Mineralfasern durch eine trilineare Momenten-Verdrehungsbeziehung erfasst werden kann. 
Die zur Darstellung dieser Beziehung notwendigen Parameter können für Träger mit I- oder Z-Querschnitt und Sandwichelemente in allen üblichen Ausführungen und Anwendungsbereichen mit einfachen Formeln ermittelt werden. Dazu werden der Elastizitätsmodul der Kernschicht, die Befestigungsart und Deckschichtdicke des Sandwichelementes, der Typ des Trägerprofils sowie die Größe der Pfettenauflast benötigt. Aus der Momenten-Verdrehungsbeziehung lässt sich in Abhängigkeit von der Auflast des Trägers die Anschlusssteifigkeit $c_{\vartheta A, k}$ als Sekantensteifigkeit ablesen und damit entsprechend DIN 18807 Teil 2 die für den Nachweis der Biegedrillknicksicherheit benötigte Drehfedersteifigkeit $\mathrm{c}_{\vartheta, \mathrm{k}}$ bestimmen.

Die Untersuchung der stabilisierenden Wirkung von zweiseitig gelagerten Schubfeldern aus Stahltrapezprofilen erfolgte mit einem Finite-Element-Modell, das sowohl durch Vergleich mit Werten der DIN 18807 Teil 1 zugrunde liegenden Schubfeldtheorie als auch durch Schubfeldversuche mit zwei- und vierseitiger Lagerung verifiziert wurde. Beim Vergleich mit den experimentellen Ergebnissen wurden die zuvor in Querzugversuchen ermittelten Nachgiebigkeiten der Verbindungsmittel am Querrand idealisiert und in der Finite-Element-Berechnung berücksichtigt. Parametrische Berechnungen an gebräuchlichen Profilgeometrien ergaben, dass die Schubsteifigkeit bei zweiseitiger Lagerung an den Querrändern aus der Schubsteifigkeit bei allseitiger Lagerung durch Abminderungsfaktoren ermittelt werden kann. Die Größe der Abminderung hängt dabei sowohl vom Seitenverhältnis des Schubfeldes als auch von der Nachgiebigkeit der Verbindungsmittel am Querrand und der Anzahl und Ausbildung der Längsstoßverbindungen ab. Die Schubsteifigkeit der untersuchten Schubfelder mit zweiseitiger Lagerung wurde auf dem Niveau der Bemessungslasten für vierseitig gelagerte Schubfelder ermittelt. Das in den Finite-ElementBerechnungen ermittelte und durch die Versuche bestätigte Last-Verformungsverhalten war bis zu dieser Laststufe stets linear, und die experimentellen und rechnerischen Grenztragfähigkeiten der untersuchten Fälle betrugen bei abnehmender Steifigkeit ein Vielfaches dieser Bemessungslasten.

\subsection{Ausblick}

Die Untersuchungen zur drehstabilisierenden Wirkung von Sandwichelementen beschränkten sich auf I-Träger und dünnwandige Pfettenprofile mit Z-Querschnitt. Außer diesen Profiltypen werden jedoch insbesondere bei Wandaufbauten mit Sandwichelementen auch häufig U-Profile als Wandriegel verwendet. Die im Rahmen der vorliegenden Untersuchungen angesetzten Randbedingungen sind in Bezug auf die Drehachse bei diesen Profilen jedoch nicht zutreffend, da sich mit deren exzentrischer Lage des Schubmittelpunktes auch das Torsionsverhalten ändert.

Die Berechnungsvorschrift zur Drehbettung von Sandwichelementen setzt für die Trägerprofile voraus, dass die Profilverformung vernachlässigt werden kann. Wenn Profilverformungen auftreten, ist im Gegensatz zu der Darstellung nach DIN 18807 Teil 2 mit einer Kopplung der Effekte aus Anschlusssteifigkeit und Profilverformung zu rechnen. Dieser Sachverhalt bedarf weitergehender Untersuchungen. 


\section{Schrifttum}

[1] ANSYS Release 8.1; Manuals; ANSYS Inc., CAD-FEM GmbH, München, 2003

[2] Baehre, R., Wolfram, R.: Zur Schubfeldberechnung von Trapezprofilen. Stahlbau 55 (1986), S. 175-179

[3] Beck, H., Reuter, M.: Setzbolzen im Stahlbau, In: Kuhlmann, U., (Hrsg.) Stahlbau-Kalender 2005, Verlag Ernst \& Sohn, Berlin, 2005

[4] Beuth-Kommentare Stahlbauten, Kommentar zu DIN 18800-1, bis -4, Ausgabe 1998-05.

[5] Bryan, E.R.: The Stressed Skin Design of Steel Buildings. Constrado Monographs, Crosby Lockwood Staples, London, 1972

[6] Burkhardt, S.: Zeitabhängiges Verhalten von Sandwichelementen mit Metalldeckschichten und Stützkernen aus Polyurethanhartschaumstoffen. Dissertation, Karlsruhe, 1988

[7] Chwalla, E.: Über die Kippstabilität querbelasteter Druckstäbe mit einfach symmetrischem Querschnitt. Federhoff-Girkmann-Festschrift Wien, Verlag Franz Deuticke, 1950

[8] Davies, J.M., Bryan, E.R.: Manual of Stressed Skin Diaphragm Design. Granada, London, 1982

[9] Deutsches Institut für Bautechnik: Allgemeine bauaufsichtliche Zulassung Z-10.4-235, Sandwichelemente mit einem Stützkern aus Mineralfaserplatten und Deckschichten aus Stahl, September 2004

[10] Deutsches Institut für Bautechnik: Allgemeine bauaufsichtliche Zulassung Z-10.4-273, Sandwichelemente mit PUR-Schaumkern und Stahldeckschichten Typ SIP W und SIP D, Mai 2002

[11] Deutsches Institut für Bautechnik: Allgemeine bauaufsichtliche Zulassung Z-10.4-342, Sandwichelemente mit einem Stützkern aus Mineralfaserplatten und Deckschichten aus Stahl, Zulassungsentwurf 2006

[12] Deutsches Institut für Bautechnik: Allgemeine bauaufsichtliche Zulassung Z-10.4-345, Sandwichelemente mit PUR-Schaumkern und Stahldeckschichten für Dach und Wand, März 2005

[13] Deutsches Institut für Bautechnik: Allgemeine bauaufsichtliche Zulassung Z-14.1-407, Verbindungselemente zur Verwendung bei Konstruktionen mit Sandwichelementen, Juni 2001

[14] Deutsches Institut für Bautechnik: Allgemeine bauaufsichtliche Zulassung Z-14.1-4, Verbindungselemente zur Verbindung von Bauteilen im Metallleichtbau, September 2005

[15] Deutsches Institut für Bautechnik: Anpassungsrichtlinie Stahlbau, „Mitteilungen" Sonderheft 11/2, 3. Auflage, Dezember 1998, einschließlich Änderungen und Ergänzungen der Anpassungsrichtlinie Stahlbau - Ausgabe Dezember 2001. DIBt Mitteilungen 1/2002

[16] Dürr, M., Kathage, K., Saal, H.: Schubsteifigkeit zweiseitig gelagerter Stahltrapezbleche. Stahlbau 75 (2006), S. 280-286 
[17] Dürr, M., Podleschny, R., Saal, H.: Untersuchungen zur Drehbettung von biegedrillknickgefährdeten Trägern durch Sandwichelemente. Stahlbau 76 (2007), S. 401-407

[18] Dürr, M., Saal, H.: Investigation of the shear stiffness of profiled steel sheeting diaphragms with only two edges fastened, In: University of Missouri-Rolla (edit.), Proceedings of the $18^{\text {th }}$ International Specialty Conference on Coldformed Steel Structures, Orlando, Florida USA, 2006

[19] European Convention for Constructional Steelwork - Technical Committee 7: European Recommendations for the Application of Metal Sheeting acting as a Diaphragm, ECCS publication No. 88, Brüssel, 1995

[20] Ewert, E., Banke, F., Schulz, U., Wolters, M.: Untersuchungen zum Knittern von Sandwichelementen mit ebenen und gesickten Deckschichten. Stahlbau 70 (2001), S. 453-463

[21] Fischer, M.: Zum Kipp-Problem von kontinuierlich seitlich gestützten ITrägern. Stahlbau 22 (1976), S. 120-124

[22] Gehring, A., Saal, H.: Kennwertermittlung für die Simulation des Walzprofilierens von Dünnblech, In: Borsutzki, M., Geisler, S. (Hrsg.) Fortschritte der Kennwertermittlung für Forschung und Praxis, Verlag Stahleisen $\mathrm{GmbH}$, Düsseldorf 2006

[23] Heil, W.: Stabilisierung von biegedrillknickgefährdeten Trägern durch Trapezblechscheiben. Stahlbau 63 (1994), S. 169-178

[24] Huan Lu Zhao: Beitrag zur Berechnung der Knitterspannungen inhomogener oder anisotroper Sandwichplatten, Fortschritts-Berichte, VDI-Reihe 18, VDIVerlag, Düsseldorf, 1993

[25] Industrieverband für das Bauen mit Stahlblech e.V. (Hrsg.), IFBS-Info 3.01: Stahltrapezprofile - Produktübersicht, Ausgabe 2003, Düsseldorf, 2003

[26] Industrieverband für das Bauen mit Stahlblech e.V. (Hrsg.), IFBS-Info 3.03: Stahl-Polyurethan(PUR)-Sandwichelemente Dach und Wand, Ausgabe 2005, Düsseldorf, Januar 2005

[27] Jungbluth, O.: Verbund- und Sandwichtragwerke. Springer Verlag, 1986

[28] Katnam, K.B., Van Impe, R., Lagae, G., De Strycker, M.: Modelling of coldformed steel sandwich purlin-sheeting systems to estimate the rotational restraint. Thin-Walled Structures 45 (2007), S. 584-590

[29] Kindmann, R., Muszkiewicz, R.: Verzweigungslasten und Eigenformen seitlich gestützter Biegeträger unter Berücksichtigung der Drehbettung. Stahlbau 71 (2002), S. 748-759

[30] Kindmann, R., Muszkiewicz, R.: Biegedrillknickmomente und Eigenformen von Biegeträgern unter Berücksichtigung der Drehbettung. Stahlbau 73 (2004), S. 98-106

[31] Klöppel, K., Unger, B.: Kippen von Durchlaufträgern bei seitlich und gegen Verdrehen elastisch gelagertem Obergurt. Stahlbau 15 (1969), Seite 203-207

[32] Koschade, R.: Die Sandwichbauweise. Verlag Ernst \& Sohn, Berlin, 2000

[33] Lindner, J.: Stabilisierung von Trägern durch Trapezbleche. Stahlbau 56 (1987), S. 9-15 
[34] Lindner, J.: Stabilisierung von Biegeträgern durch Drehbettung - eine Klarstellung. Stahlbau 56 (1987), S. 365-373

[35] Lindner, J., Gregull, T.: Drehbettungswerte für Dacheindeckungen mit unterlegter Wärmedämmung. Stahlbau 58 (1989), S. 173-179

[36] Lindner, J., Groeschel, F.: Drehbettungswerte für die Profilblechbefestigung mit Setzbolzen bei unterschiedlich großen Auflasten. Stahlbau 65 (1996), S. 218-224

[37] Lindner, J., Kurt, W.: Drehbettungswerte bei Unterwind. Bauingenieur 55 (1980), S. 365-369

[38] Lindner, J., Seidel, F.: Stabilisierung von Biegeträgern durch Trapezprofile ohne Schubfeldausbildung, Vortrag beim 25. Steinfurter Stahlbauseminar, FH Münster, Rheine, Mai 2006

[39] Ministerium für Bauen und Verkehr des Landes Nordrhein-Westfalen: Bescheid Nr. VI A 3-543-996, Querschnitts- und Tragfähigkeitswerte der Stahltrapezprofile SP59/900, SP80/830, SP105/1035, SP111/930, SP135/930, SP153/840, SP160/750, August 2006

[40] Möller, R., Pöter, H., Schwarze, K.: Planen und Bauen mit Trapezprofilen und Sandwichelementen; Band 1: Grundlagen, Bauweisen, Bemessung mit Beispielen, Verlag Ernst \& Sohn, Berlin, 2004

[41] Osterrieder, P., Voigt, M., Saal, H.: Zur Neuregelung des Biegedrillknicknachweises nach EDIN 18800 Teil 2 (Ausgabe März 1988). Stahlbau 58 (1989), S. 341-347

[42] Oxfort, J., Hildenbrand, P.: Traglastversuch an durchlaufenden Pfetten mit Leichtbetonplatten als Dacheindeckung. Bauingenieur 46 (1971), S. 131-135

[43] Pelikan, W.: Versuche zur Ermittlung der Kippsicherheit von Stahlpfetten mit Welleternit-Eindeckung. Bauingenieur 40 (1965), S. 55-59

[44] Pelikan, W.: Traglastversuche mit kontinuierlichen Pfetten und WelleternitEindeckung. Bauingenieur 41 (1966), S. 440-444

[45] Riedeburg, K.: Untersuchungen zum wirtschaftlichen Einsatz von Sandwichelementen in Dächern und Wänden, Abschlussbericht zur Forschungsaufgabe, Leipzig, 1994

[46] Ringel, N.: Untersuchungen zur aussteifenden Wirkung von Schubfeldern auf stabilitätsgefährdete Träger, Diplomarbeit, Lehrstuhl für Stahl- und Leichtmetallbau, Universität Karlsruhe (TH), Oktober 2005

[47] Roik, K., Carl, J., Lindner, J.: Biegetorsionsprobleme gerader dünnwandiger Stäbe. Verlag von Wilhelm Ernst \& Sohn, 1972

[48] Sauer, R., Wagner, W.: Experimentelle und numerische Untersuchungen zur aussteifenden Wirkung von Trapezblechscheiben. Stahlbau 64 (1995), S. $289-294$

[49] Schardt, R., Strehl, C.: Stand der Theorie zur Bemessung von Trapezblechscheiben. Stahlbau 49 (1980), S. 325-334

[50] Schwarze, K., Lohmann, F.A.: Konstruktion und Bemessung von Dach- und Wandflächen aus Stahl, In: Kuhlmann, U., (Hrsg.) Stahlbau-Kalender 1999, Verlag Ernst \& Sohn, Berlin 1999 
[51] Stamm, K., Witte, H.: Sandwichkonstruktionen. Springer Verlag, 1978

[52] Strehl, C.: Berechnung regelmäßig periodisch aufgebauter Faltwerksquerschnitte unter Schubbelastung am Beispiel des Trapezblechs. Dissertation, Darmstadt, 1976

[53] Strehl, C.: Bestimmung der Schubsteifigkeitswerte von Trapezblechen mit Tabellen-Kalkulationsprogramm. Stahlbau 74 (2005), S. 708-716

[54] Vogel, U.: Zur Kippstabilität durchlaufender Stahlpfetten. Stahlbau 16 (1970), S. $76-82$

[55] Vogel, U., Heil, W.:Traglast-Tabellen, Tabellen für die Bemessung durchlaufender I-Träger mit und ohne Normalkraft nach dem Traglastverfahren (DIN 18800, Teil 2). 3. Auflage, Stahl-Eisen-Verlag, 1993

[56] Wolters, M., Banke, F., Ewert, E., Schulz, U.: Untersuchungen zum Knittern von imperfekten Sandwichelementen, Stahlbau 71 (2002), S. 253-262

[57] Unveröffentlichte Berichte:

Versuchsanstalt für Stahl, Holz und Steine, Universität Karlsruhe (TH):

[a] Bericht Nr. 054003, Prüfung von Brucha-Isolierdachpaneele und BruchaIsolierwandpaneele hergestellt nach allgemeiner bauaufsichtlicher Zulassung Z-10.4-323, Juni 2005

Institut für Stahlbau Leipzig GmbH:

[b] Prüfbericht 11/96 - Teil 1, Versuche zum Tragverhalten der Dacheindeckung der $60 \mathrm{~m}$ Halle der Kompaktwerft Wismar (MTW) - Tragverhalten der Trapezprofilscheibe, November 1996 


\section{Zitierte Normen}

DIN 1025 Teil 2: Warmgewalzte I-Träger - Teil 2: I-Träger, IPB-Reihe; Maße, Masse, statische Werte, November 1995

DIN 1026 Teil 1: Warmgewalzter U-Profilstahl - Teil 1: Warmgewalzter U-Profilstahl mit geneigten Flanschflächen; Maße, Masse und statische Werte, März 2000

DIN 4114: Stabilitätsfälle, Knickung, Kippung, Beulung, Berechnungsgrundlagen, Juli 1952

DIN 18800 Teil 2: Stahlbauten - Stabilitätsfälle, Knicken von Stäben und Stabwerken, November 1990

DIN 18807 Teil 1: Trapezprofile im Hochbau; Stahltrapezprofile; Allgemeine Anforderungen, Ermittlung der Tragfähigkeitswerte durch Berechnung, Juni 1987

DIN 18807 Teil 3: Trapezprofile im Hochbau; Stahltrapezprofile; Festigkeitsnachweis und konstruktive Ausbildung, Juni 1987

DIN EN 826: Wärmedämmstoffe für das Bauwesen - Bestimmung des Verhaltens bei Druckbeanspruchung, Mai 1996

DIN EN 1602: Wärmedämmstoffe für das Bauwesen - Bestimmung der Rohdichte, Januar 1997

DIN EN 1607: Wärmedämmstoffe für das Bauwesen - Bestimmung der Zugfestigkeit senkrecht zur Plattenebene, Januar 1997

DIN EN 1993-1-3: Eurocode 3: Bemessung und Konstruktion von Stahlbauten - Teil 1-3: Allgemeine Regeln - Ergänzende Regeln für kaltgeformte dünnwandige Bauteile und Bleche, Februar 2007

DIN EN 10002 Teil 1: Metallische Werkstoffe - Zugversuch - Teil 1: Prüfverfahren bei Raumtemperatur, Dezember 2001

DIN EN 10326: Kontinuierlich schmelztauchveredeltes Band und Blech aus Baustählen - Technische Lieferbedingungen, September 2004

DIN EN 14509: Selbsttragende Sandwich-Elemente mit beidseitigen Metalldeckschichten - Werkmäßig hergestellte Produkte - Spezifikationen, Februar 2007

DIN EN ISO 291: Kunststoffe - Normalklimate für Konditionierung und Prüfung (ISO 291:2005), Februar 2006

Entwurf zu DIN 18807 Teil 4: Trapezprofile im Hochbau; Verbindungen zwischen Stahltrapezprofilen und Bauteilen aus Stahl; Ermittlung der Tragfähigkeit durch Versuche, 1986

Entwurf zu DIN EN ISO 6892: Metallische Werkstoffe - Zugversuch - Prüfverfahren bei Raumtemperatur (ISO/DIS 6892:2007), Mai 2007

ISO 12491: Statistische Verfahren für die Güteüberwachung von Bauprodukten und Bauteilen, Mai 1997 


\section{Anhang}

Anhang A1: Zusammenstellung der Versuchsreihen Drehbettung 106

Anhang A2: Momenten-Verdrehungsbeziehungen der Versuchsreihen Drehbettung ....115

Anhang A3: Ergebnisse der Zugversuche an den Deckschichten 132

Anhang A4: Ergebnisse der Untersuchungen der Sandwich-Kernschichten 133

Anhang A5: statistische Auswertungen 


\section{Anhang A1: Zusammenstellung der Versuchsreihen Drehbettung}

\section{Versuch D1/PUR:}

\author{
Element \\ Zulassungs-Nr. \\ durchgehende Elementdicke \\ Deckschichtdicke oben \\ Deckschichtdicke unten \\ Elementlänge \\ Stützweite
}

Pfettenprofil

Verschraubung mit Unterkonstruktion

Art

Typ

Abstände quer

Dichtscheiben-Durchmesser

Verschraubung am Längsstoß

Typ

Abstände

Dichtscheiben-Durchmesser
Hoesch Thermodach TL75

Z-10.4-345

$40 \mathrm{~mm}$

$0,50 \mathrm{~mm}$

$0,40 \mathrm{~mm}$

$4000 \mathrm{~mm}$

$3500 \mathrm{~mm}$

IPE160

Tiefsickenverschraubung

EJOT JZ3-6,3×64

2,5 x Untergurtbreite entspricht 2 Schrauben/Element

EJOT JT3-2H-5,5×25

$500 \mathrm{~mm}$

$16 \mathrm{~mm}$

\section{$16 \mathrm{~mm}$}

\section{Versuch D2/PUR:}

Element

Zulassungs-Nr.

durchgehende Elementdicke

Deckschichtdicke oben

Deckschichtdicke unten

Elementlänge

Stützweite

Pfettenprofil

Verschraubung mit Unterkonstruktion

Art

Typ

Abstände quer

Dichtscheiben-Durchmesser

Verschraubung am Längsstoß

Typ

Abstände

Dichtscheiben-Durchmesser

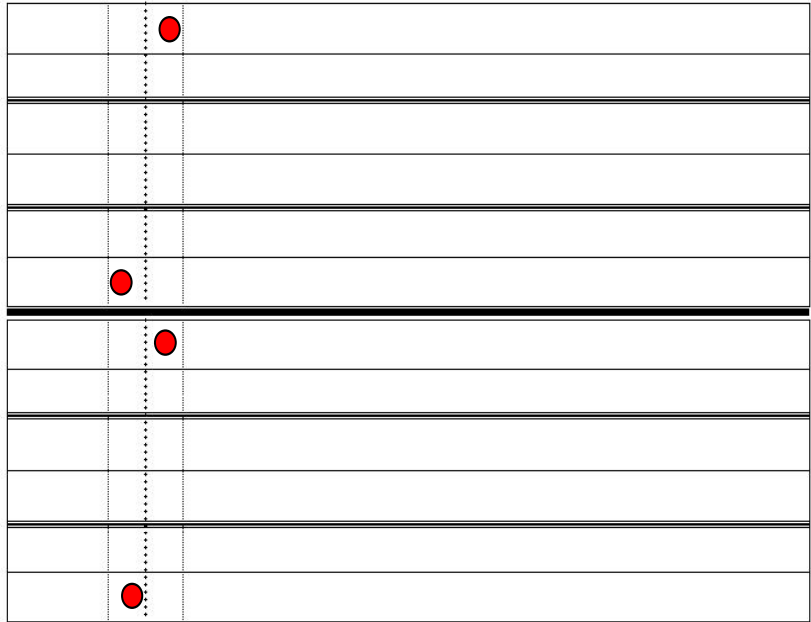

Z-10.4-345

$40 \mathrm{~mm}$

$0,75 \mathrm{~mm}$

$0,55 \mathrm{~mm}$

$4000 \mathrm{~mm}$

$3500 \mathrm{~mm}$

IPE160

Tiefsickenverschraubung

$$
\text { EJOT JZ3-6,3×64 }
$$

2,0 x Untergurtbreite entspricht 2 Schrauben/Element

$$
22 \mathrm{~mm}
$$

\begin{tabular}{|l|c:c|l|}
\hline & & \\
\hline & 0 & \\
\hline \hline & & \\
\hline & & \\
\hline \hline & 0 & \\
\hline & 0 & \\
\hline \hline & $\vdots$ & \\
\hline & & & \\
\hline \hline & $\vdots$ & \\
\hline & & \\
\hline \hline & 0 & \\
\hline & $\vdots$ & \\
\hline
\end{tabular}

EJOT JT3-2H-5,5×25

$500 \mathrm{~mm}$

$16 \mathrm{~mm}$ 


\section{Versuch D3/PUR:}

Element

Zulassungs-Nr.

durchgehende Elementdicke

Deckschichtdicke oben

Deckschichtdicke unten

Elementlänge

Stützweite

Pfettenprofil
Hoesch Thermodach TL95

Z-10.4-345

$60 \mathrm{~mm}$

$0,75 \mathrm{~mm}$

$0,55 \mathrm{~mm}$

$4000 \mathrm{~mm}$

$3500 \mathrm{~mm}$

IPE160

Verschraubung mit Unterkonstruktion

Art

Typ

Abstände quer

Dichtscheiben-Durchmesser

Tiefsickenverschraubung

EJOT JZ3-6,3×90

2,0 x Untergurtbreite entspricht 2 Schrauben/Element

$22 \mathrm{~mm}$

EJOT JT3-2H-5,5×25

$500 \mathrm{~mm}$

$16 \mathrm{~mm}$

Lăngsstoß

Abstände
Dichtscheiben-Durchmesser

\section{Versuch D4/PUR:}

Element

Zulassungs-Nr.

durchgehende Elementdicke

Deckschichtdicke oben

Deckschichtdicke unten

Elementlänge

Stützweite

Pfettenprofil

Hoesch Thermodach TL75

Z-10.4-345

$40 \mathrm{~mm}$

$0,50 \mathrm{~mm}$

$0,40 \mathrm{~mm}$

$3000 \mathrm{~mm}$

$2500 \mathrm{~mm}$

IPE160

Verschraubung mit Unterkonstruktion

Art

Typ

Abstände quer

Dichtscheiben-Durchmesser

Verschraubung am Längsstoß

Typ

Abstände

Dichtscheiben-Durchmesser

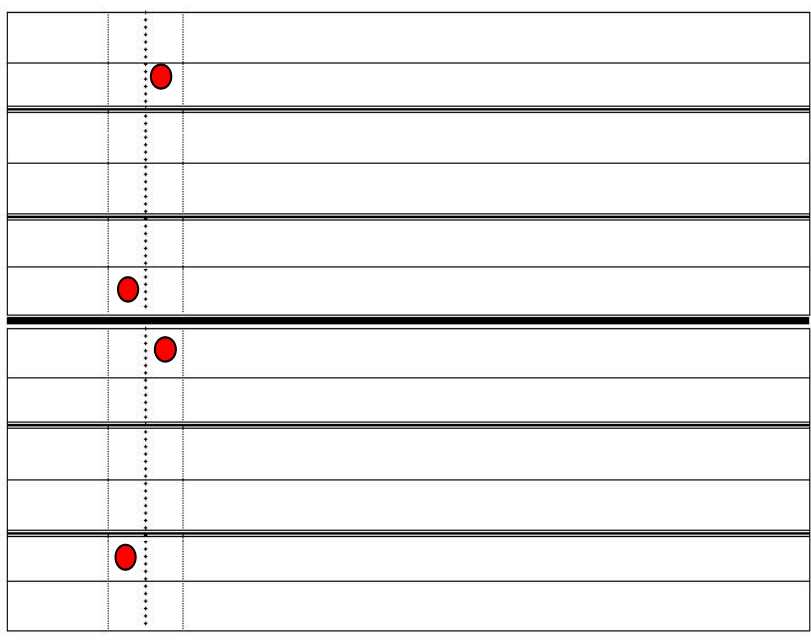

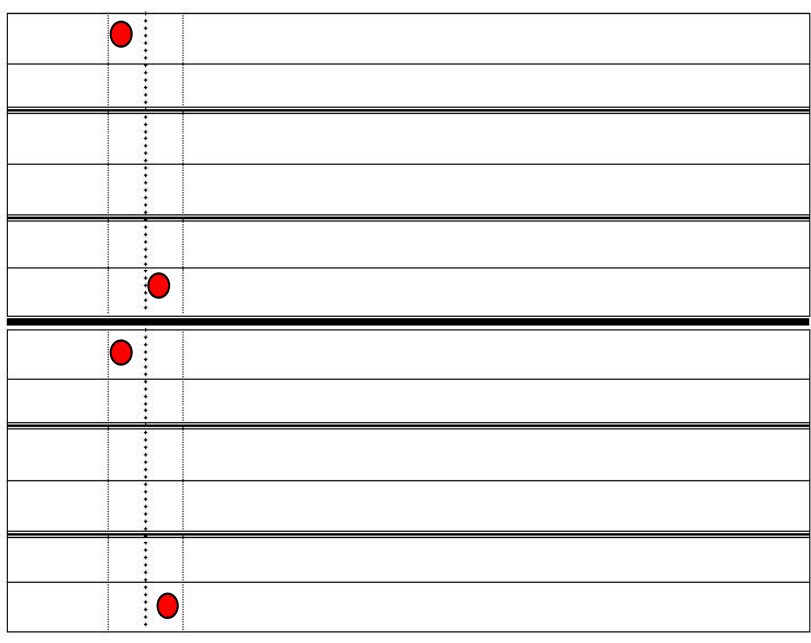

Tiefsickenverschraubung

EJOT JZ3-6,3x64

2,5 x Untergurtbreite entspricht 2 Schrauben/Element

$16 \mathrm{~mm}$

EJOT JT3-2H-5,5×25

$500 \mathrm{~mm}$

$16 \mathrm{~mm}$ 


\section{Versuch D5/PUR:}

\author{
Element \\ Zulassungs-Nr. \\ durchgehende Elementdicke \\ Deckschichtdicke oben \\ Deckschichtdicke unten \\ Elementlänge \\ Stützweite
}

Pfettenprofil

Verschraubung mit Unterkonstruktion

Art

Typ

Abstände quer

Dichtscheiben-Durchmesser

Verschraubung am Längsstoß

Typ

Abstände

Dichtscheiben-Durchmesser
Hoesch Thermodach TL75

Z-10.4-345

$40 \mathrm{~mm}$

$0,75 \mathrm{~mm}$

$0,55 \mathrm{~mm}$

$3000 \mathrm{~mm}$

$2500 \mathrm{~mm}$

IPE160

Tiefsickenverschraubung

$$
\text { EJOT JZ3-6,3×64 }
$$

2,0 x Untergurtbreite entspricht 2 Schrauben/Element

$$
22 \mathrm{~mm}
$$

EJOT JT3-2H-5,5x25

$500 \mathrm{~mm}$

$16 \mathrm{~mm}$

\section{Versuch D6/PUR:}

\author{
Element \\ Zulassungs-Nr. \\ durchgehende Elementdicke \\ Deckschichtdicke oben \\ Deckschichtdicke unten \\ Elementlänge \\ Stützweite
}

Pfettenprofil
Hoesch Thermodach TL95

Z-10.4-345

$60 \mathrm{~mm}$

$0,75 \mathrm{~mm}$

$0,55 \mathrm{~mm}$

$3000 \mathrm{~mm}$

$2500 \mathrm{~mm}$

IPE160

Verschraubung mit Unterkonstruktion

Art

Typ

Abstände quer

Dichtscheiben-Durchmesser

Tiefsickenverschraubung

EJOT JZ3-6,3×90

2,0 x Untergurtbreite entspricht 2 Schrauben/Element

$22 \mathrm{~mm}$

EJOT JT3-2H-5,5×25

$500 \mathrm{~mm}$

Abstände

$16 \mathrm{~mm}$

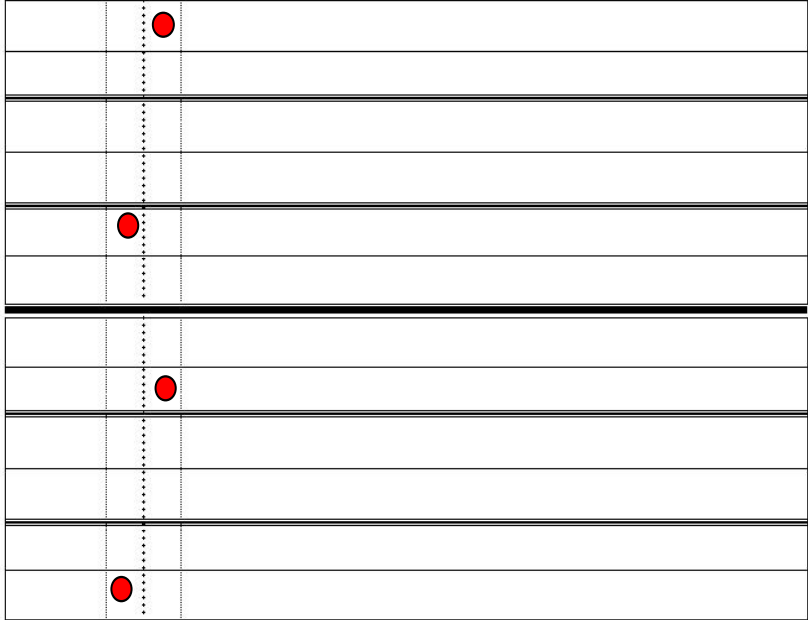




\section{Versuch W1/PUR:}

\author{
Element \\ Zulassungs-Nr. \\ durchgehende Elementdicke \\ Deckschichtdicke oben \\ Deckschichtdicke unten \\ Elementlänge \\ Stützweite
}

Pfettenprofil
Salzgitter SIP W40 SB

Z-10.4-273

$40 \mathrm{~mm}$

$0,60 \mathrm{~mm}$

$0,50 \mathrm{~mm}$

$3400 \mathrm{~mm}$

$3000 \mathrm{~mm}$

IPE160

Verschraubung mit Unterkonstruktion

Art

Anordnung

Typ

Randabstand $\mathrm{e}_{\mathrm{R}}$

Dichtscheiben-Durchmesser

Verschraubung am Längsstoß

Typ

Abstände

Dichtscheiben-Durchmesser
$50 \mathrm{~mm}$

$16 \mathrm{~mm}$

EJOT JT3-2H-5,5x25

$500 \mathrm{~mm}$

$16 \mathrm{~mm}$
EJOT JZ3-6,3×64

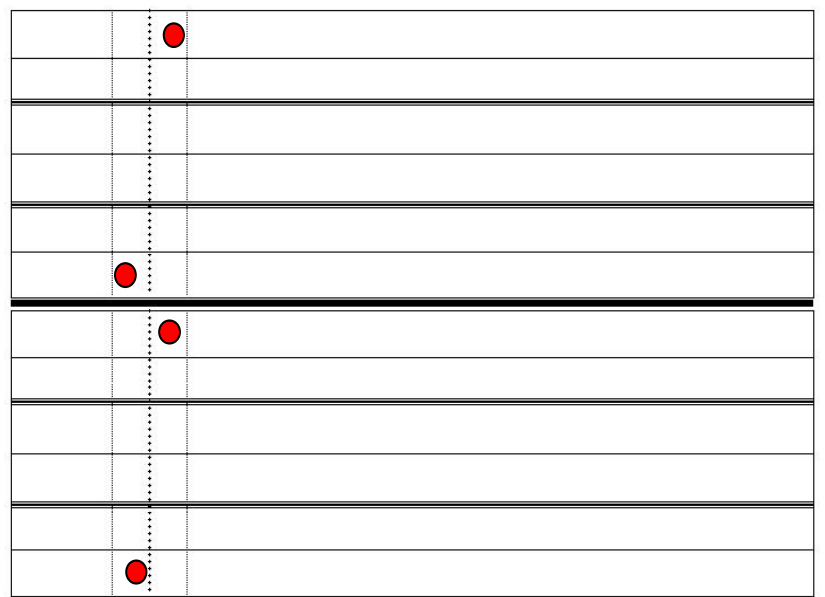

\section{Versuch W2/PUR:}

Element

Zulassungs-Nr.

durchgehende Elementdicke

Deckschichtdicke oben

Deckschichtdicke unten

Elementlänge

Stützweite

Pfettenprofil

Verschraubung mit Unterkonstruktion

Art

Anordnung

Typ

Randabstand $\mathrm{e}_{\mathrm{R}}$

Dichtscheiben-Durchmesser

Verschraubung am Längsstoß

Typ

Abstände

Dichtscheiben-Durchmesser
Salzgitter SIP W40 SB

Z-10.4-273

$40 \mathrm{~mm}$

$0,60 \mathrm{~mm}$

$0,50 \mathrm{~mm}$

$3400 \mathrm{~mm}$

$3000 \mathrm{~mm}$

IPE160

direkte Verschraubung

alternierend

EJOT JZ3-6,3×64

$50 \mathrm{~mm}$

$19 \mathrm{~mm}$

\begin{tabular}{|l|c:c|l|}
\hline & 0 & \\
\hline & $\vdots$ & \\
\hline \hline & $\vdots$ & \\
\hline & & \\
\hline \hline & $\vdots$ & \\
\hline & 0 & \\
\hline \hline & $\vdots$ & \\
\hline & $\vdots$ & \\
\hline \hline & $\vdots$ & \\
\hline & & \\
\hline \hline & $\vdots$ & \\
\hline & 0 & \\
\hline
\end{tabular}

EJOT JT3-2H-5,5×25

$500 \mathrm{~mm}$ $16 \mathrm{~mm}$ 


\section{Versuch W3/PUR:}

Element

Zulassungs-Nr.

durchgehende Elementdicke

Deckschichtdicke oben

Deckschichtdicke unten

Elementlänge

Stützweite

Pfettenprofil

Verschraubung mit Unterkonstruktion
Art

Anordnung

Typ

Randabstand $e_{R}$

Dichtscheiben-Durchmesser

Verschraubung am Längsstoß

Typ

Abstände

Dichtscheiben-Durchmesser
Salzgitter SIP W40 SB

Z-10.4-273

$40 \mathrm{~mm}$

$0,60 \mathrm{~mm}$

$0,50 \mathrm{~mm}$

$3400 \mathrm{~mm}$

$3000 \mathrm{~mm}$

IPE160

direkte Verschraubung

einseitig

EJOT JZ3-6,3×64

$50 \mathrm{~mm}$

$19 \mathrm{~mm}$

EJOT JT3-2H-5,5×25

$500 \mathrm{~mm}$

$16 \mathrm{~mm}$

\section{Versuch W4/PUR:}

Element

Zulassungs-Nr.

durchgehende Elementdicke

Deckschichtdicke oben

Deckschichtdicke unten

Elementlänge

Stützweite

Pfettenprofil

Verschraubung mit Unterkonstruktion

Art

Anordnung

Typ

Randabstand $\mathrm{e}_{\mathrm{R}}$

Dichtscheiben-Durchmesser

Verschraubung am Längsstoß

Typ

Abstände

Dichtscheiben-Durchmesser

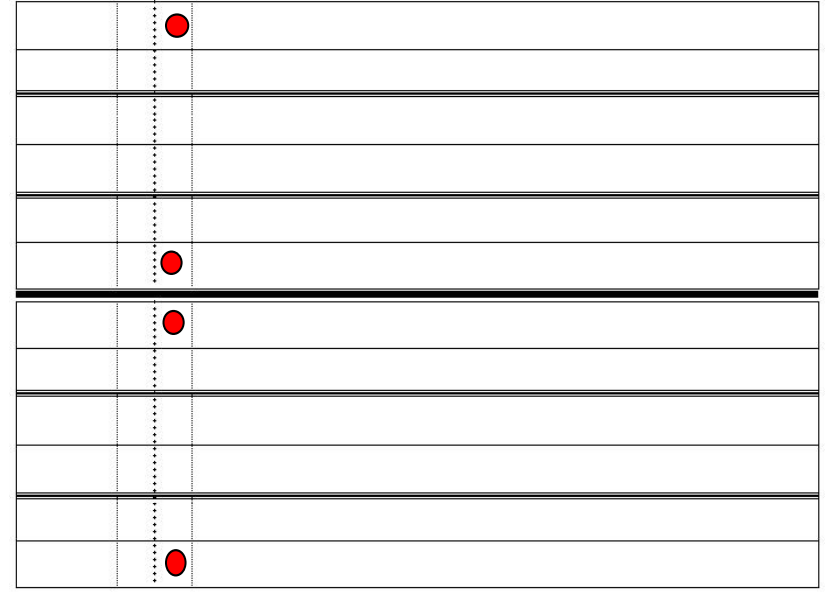

Hoesch Thermowand LL40

Z-10.4-345

$40 \mathrm{~mm}$

$0,50 \mathrm{~mm}$

$0,40 \mathrm{~mm}$

$3400 \mathrm{~mm}$

$3000 \mathrm{~mm}$

IPE160

direkte Verschraubung alternierend

EJOT JZ3-6,3×64

$50 \mathrm{~mm}$

$16 \mathrm{~mm}$

EJOT JT3-2H-5,5×25

$500 \mathrm{~mm}$

$16 \mathrm{~mm}$ 


\title{
Versuch W5/PUR:
}

Element

Zulassungs-Nr.

durchgehende Elementdicke

Deckschichtdicke oben

Deckschichtdicke unten

Elementlänge

Stützweite

Pfettenprofil

Verschraubung mit Unterkonstruktion

Art

Anordnung

Typ

Randabstand $\mathrm{e}_{\mathrm{R}}$

Dichtscheiben-Durchmesser

Verschraubung am Längsstoß

Typ

Abstände

Dichtscheiben-Durchmesser
Hoesch Thermowand LL40

Z-10.4-345

$40 \mathrm{~mm}$

$0,50 \mathrm{~mm}$

$0,40 \mathrm{~mm}$

$3400 \mathrm{~mm}$

$3000 \mathrm{~mm}$

IPE160

direkte Verschraubung

alternierend

EJOT JZ3-6,3×64

$50 \mathrm{~mm}$

$19 \mathrm{~mm}$

EJOT JT3-2H-5,5×25

$500 \mathrm{~mm}$

$16 \mathrm{~mm}$

\section{Versuch W6/PUR:}

\author{
Element \\ Zulassungs-Nr. \\ durchgehende Elementdicke \\ Deckschichtdicke oben \\ Deckschichtdicke unten \\ Elementlänge \\ Stützweite
}

Pfettenprofil

Verschraubung mit Unterkonstruktion

Art

Anordnung

Typ

Randabstand $e_{R}$

Dichtscheiben-Durchmesser

Verschraubung am Längsstoß

Typ

Abstände

Dichtscheiben-Durchmesser

\begin{tabular}{|c|c|c|}
\hline & 0 & \\
\hline & & \\
\hline & & \\
\hline & & \\
\hline & & \\
\hline 0 & & \\
\hline & 0 & \\
\hline & & \\
\hline & & \\
\hline & & \\
\hline & & \\
\hline 0 & & \\
\hline
\end{tabular}

\begin{tabular}{|l|c:c|l|}
\hline & 0 & \\
\hline & & & \\
\hline \hline & $\vdots$ & \\
\hline & $\vdots$ & \\
\hline \hline & $\vdots$ & \\
\hline & 0 & \\
\hline \hline & $\vdots$ & \\
\hline & $\vdots$ & \\
\hline \hline & $\vdots$ & \\
\hline & & \\
\hline \hline & $\vdots$ & \\
\hline & 0 & \\
\hline
\end{tabular}

direkte Verschraubung alternierend

EJOT JZ3-6,3x64

$50 \mathrm{~mm}$

$16 \mathrm{~mm}$

EJOT JT3-2H-5,5×25

$500 \mathrm{~mm}$

$16 \mathrm{~mm}$ 


\section{Versuch D1/MW:}

Element

Zulassungs-Nr.

durchgehende Elementdicke

Deckschichtdicke oben

Deckschichtdicke unten

Elementlänge

Stützweite

Pfettenprofil
Arcelor Ondafibre 3005T

Z-10.4-342

$40 \mathrm{~mm}$

$0,75 \mathrm{~mm}$

$0,50 \mathrm{~mm}$

$3000 \mathrm{~mm}$

$2700 \mathrm{~mm}$

IPE160

Verschraubung mit Unterkonstruktion

Art

Typ

Abstände quer

Dichtscheiben-Durchmesser

Verschraubung am Längsstoß

Typ

Abstände

Dichtscheiben-Durchmesser

EJOT JZ3-6,3×90

$22 \mathrm{~mm}$

EJOT JT3-2H-5,5×25

$500 \mathrm{~mm}$

$16 \mathrm{~mm}$

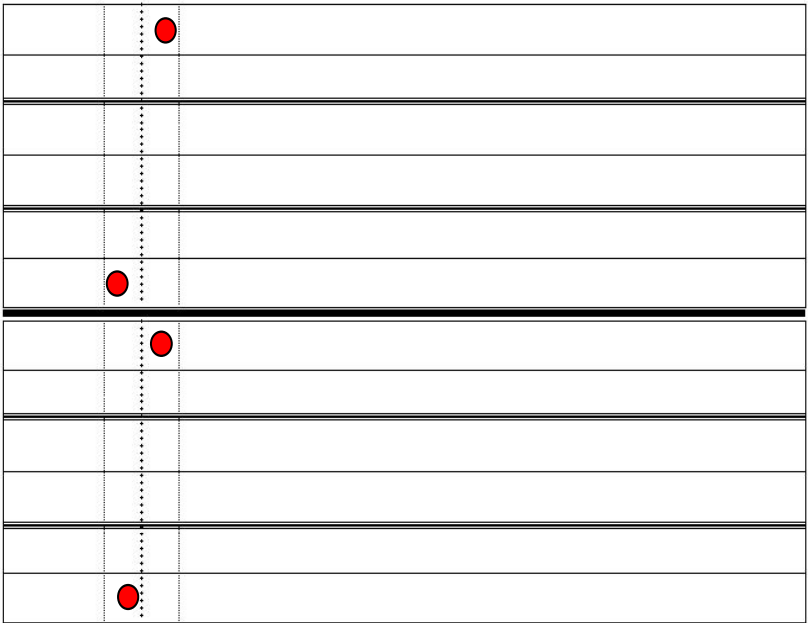

$2,5 \times$ Untergurtbreite entspricht 2 Schrauben/Element

\section{Versuch W2/MW:}

Element

Zulassungs-Nr.

durchgehende Elementdicke

Deckschichtdicke oben

Deckschichtdicke unten

Elementlänge

Stützweite

Pfettenprofil
Hoesch isorock LL 80

Z-10.4-235

$80 \mathrm{~mm}$

$0,63 \mathrm{~mm}$

$0,50 \mathrm{~mm}$

$3000 \mathrm{~mm}$

$2700 \mathrm{~mm}$

IPE160

Verschraubung mit Unterkonstruktion

Art

Anordnung

Typ

Randabstand $e_{R}$

Dichtscheiben-Durchmesser

Verschraubung am Längsstoß

Typ

Abstände

Dichtscheiben-Durchmesser direkte Verschraubung alternierend EJOT JZ3-6,3×100 $50 \mathrm{~mm}$ $19 \mathrm{~mm}$

EJOT JT3-2H-5,5×25

$500 \mathrm{~mm}$

$16 \mathrm{~mm}$

\begin{tabular}{|l|c:c|l|}
\hline & 0 & \\
\hline & $\vdots$ & \\
\hline \hline & $\vdots$ & \\
\hline & $\vdots$ & \\
\hline \hline & $\vdots$ & \\
\hline & 0 & \\
\hline \hline & $\vdots$ & \\
\hline & $\vdots$ & \\
\hline \hline & $\vdots$ & \\
\hline & $\vdots$ & \\
\hline \hline & $\vdots$ & \\
\hline & 0 & \\
\hline
\end{tabular}




\section{Versuch D7a/PUR:}

Element

Zulassungs-Nr.

durchgehende Elementdicke

Deckschichtdicke oben

Deckschichtdicke unten

Elementlänge

Stützweite

Pfettenprofil
Hoesch Thermodach TL95

Z-10.4-345

$60 \mathrm{~mm}$

$0,75 \mathrm{~mm}$

$0,55 \mathrm{~mm}$

$3000 \mathrm{~mm}$

$2500 \mathrm{~mm}$

IPE160

Verschraubung mit Unterkonstruktion

Art

Typ

Tiefsickenverschraubung

EJOT JZ3-6,3×90

Abstände quer

Dichtscheiben-Durchmesser

Verschraubung am Längsstoß

Typ

Abstände

JOT JT3-2H-5,5×25

$500 \mathrm{~mm}$

$16 \mathrm{~mm}$

\section{$22 \mathrm{~mm}$}

\section{Versuch D7b/PUR:}

Element

Zulassungs-Nr.

durchgehende Elementdicke

Deckschichtdicke oben

Deckschichtdicke unten

Elementlänge

Stützweite

Pfettenprofil
Hoesch Thermodach TL95

Z-10.4-345

$60 \mathrm{~mm}$

$0,75 \mathrm{~mm}$

$0,55 \mathrm{~mm}$

$3000 \mathrm{~mm}$

$2500 \mathrm{~mm}$

IPE160

Verschraubung mit Unterkonstruktion
Art

Typ

Abstände quer

Dichtscheiben-Durchmesser

Verschraubung am Längsstoß

Typ

Abstände

Dichtscheiben-Durchmesser

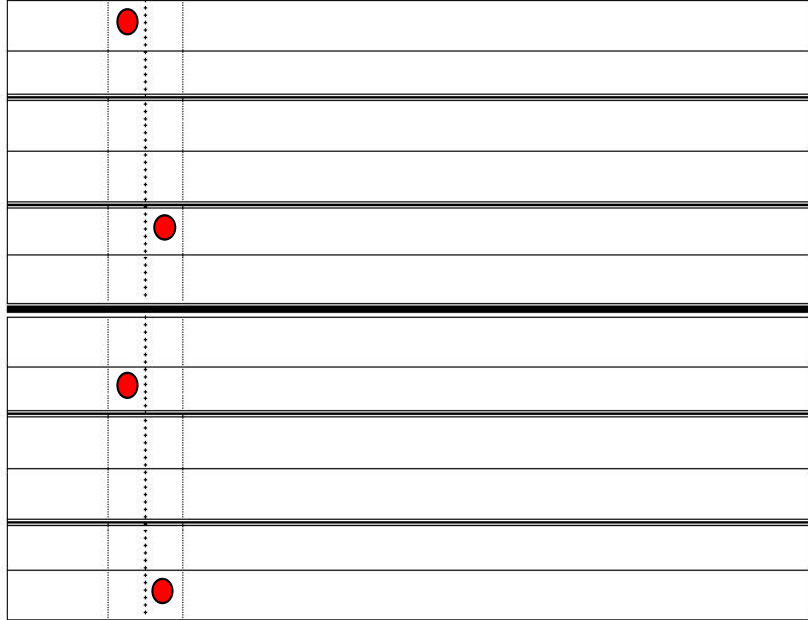

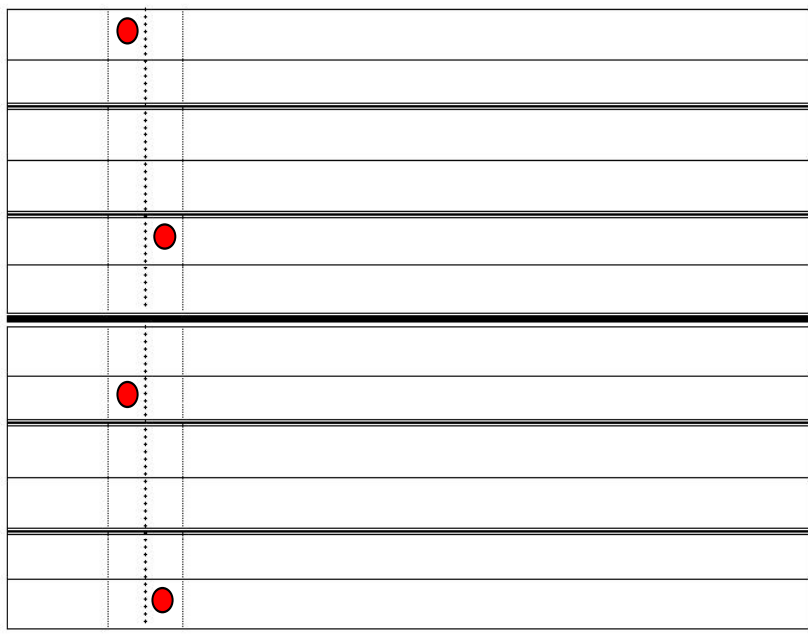

Tiefsickenverschraubung (gelöst)

EJOT JZ3-6,3×90

2,0 x Untergurtbreite entspricht 2 Schrauben/Element

$22 \mathrm{~mm}$

EJOT JT3-2H-5,5×25

$500 \mathrm{~mm}$

$16 \mathrm{~mm}$ 


\section{Versuch DZIPUR:}

\section{Element}

Zulassungs-Nr.

durchgehende Elementdicke

Deckschichtdicke oben

Deckschichtdicke unten

Elementlänge

Stützweite

Pfettenprofil
Hoesch Thermodach TL75

Z-10.4-345

$40 \mathrm{~mm}$

$0,50 \mathrm{~mm}$

$0,40 \mathrm{~mm}$

$3000 \mathrm{~mm}$

$2500 \mathrm{~mm}$

Z140-65

Verschraubung mit Unterkonstruktion

Art

Typ

Abstände quer

Dichtscheiben-Durchmesser

Verschraubung am Längsstoß

Typ

Abstände

Dichtscheiben-Durchmesser

EJOT JZ3-6,3

$16 \mathrm{~mm}$

EJOT JT3-2H-5,5×25

$500 \mathrm{~mm}$

$16 \mathrm{~mm}$

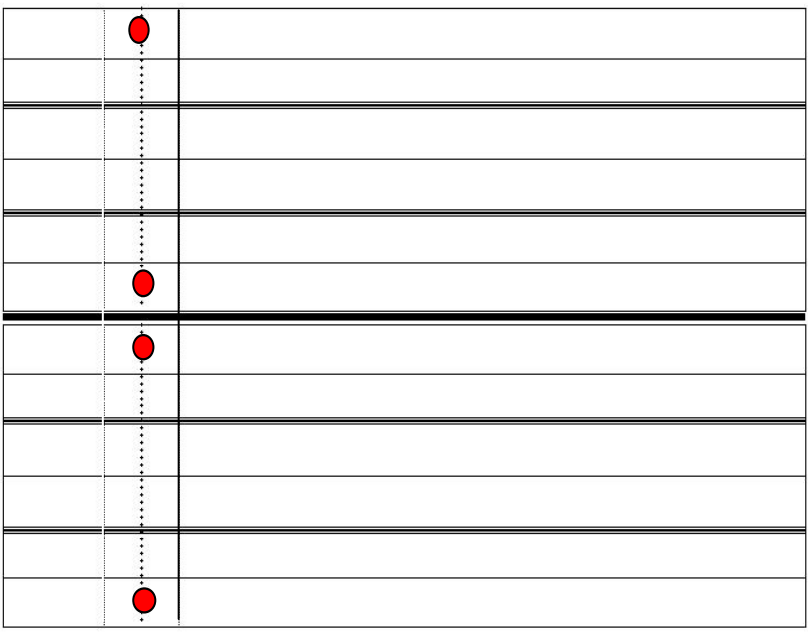

2,5 x Untergurtbreite entspricht 2 Schrauben/Element 


\section{Anhang A2: Momenten-Verdrehungsbeziehungen der Versuchsreihen Drehbettung}
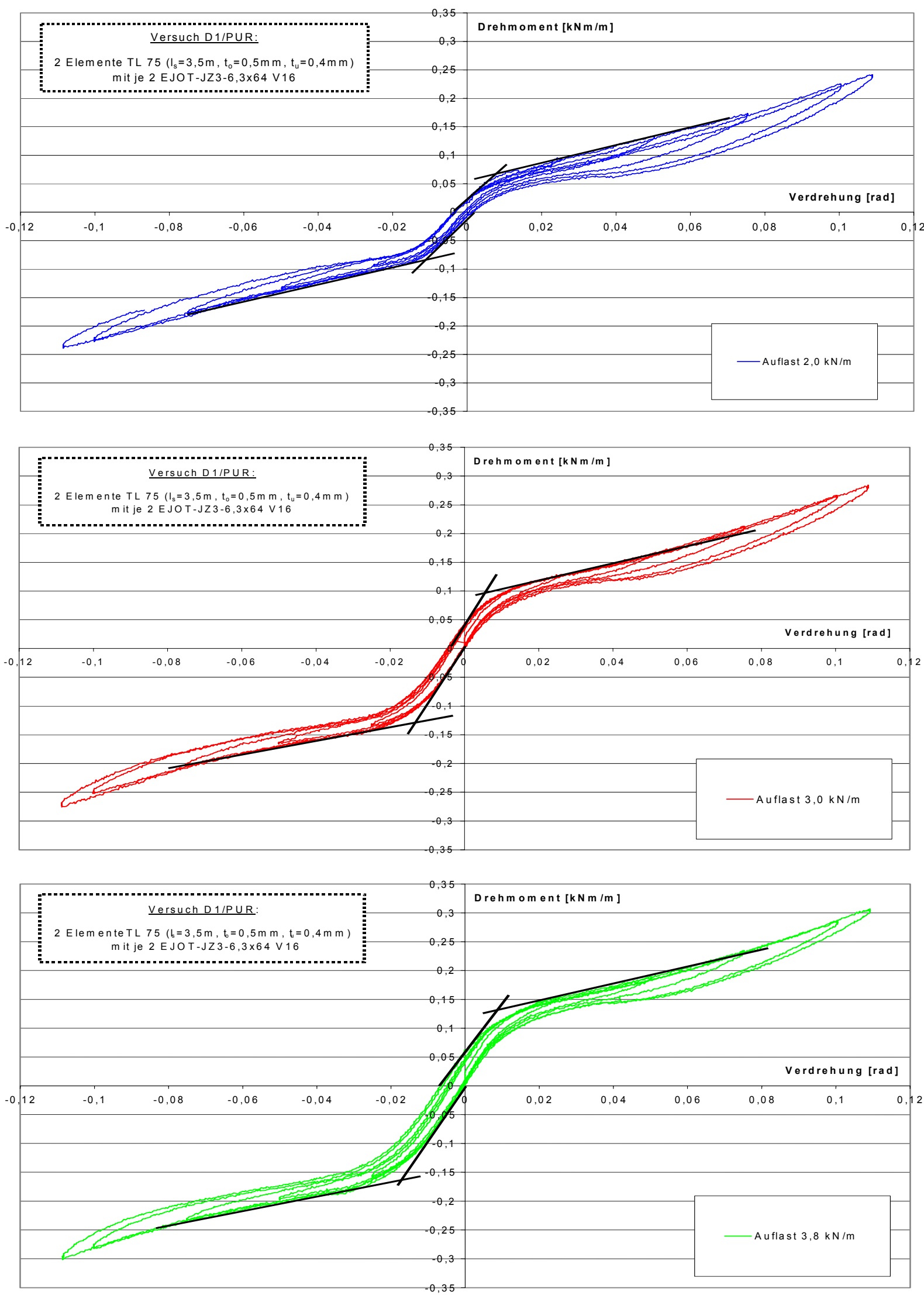

Diagramm A1: Momenten-Verdrehungsbeziehungen des Versuchs D1/PUR 

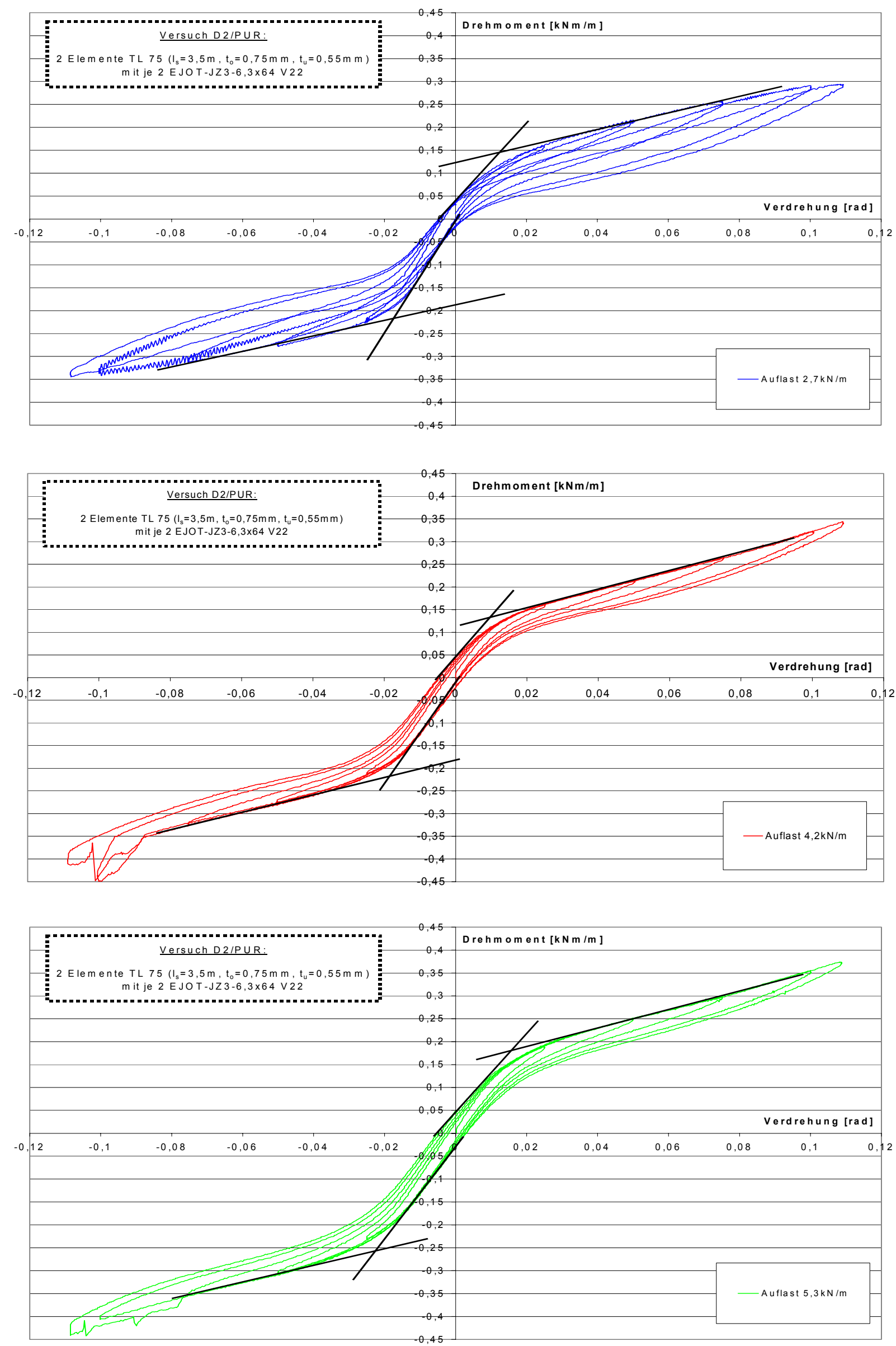

Diagramm A2: Momenten-Verdrehungsbeziehungen des Versuchs D2/PUR 

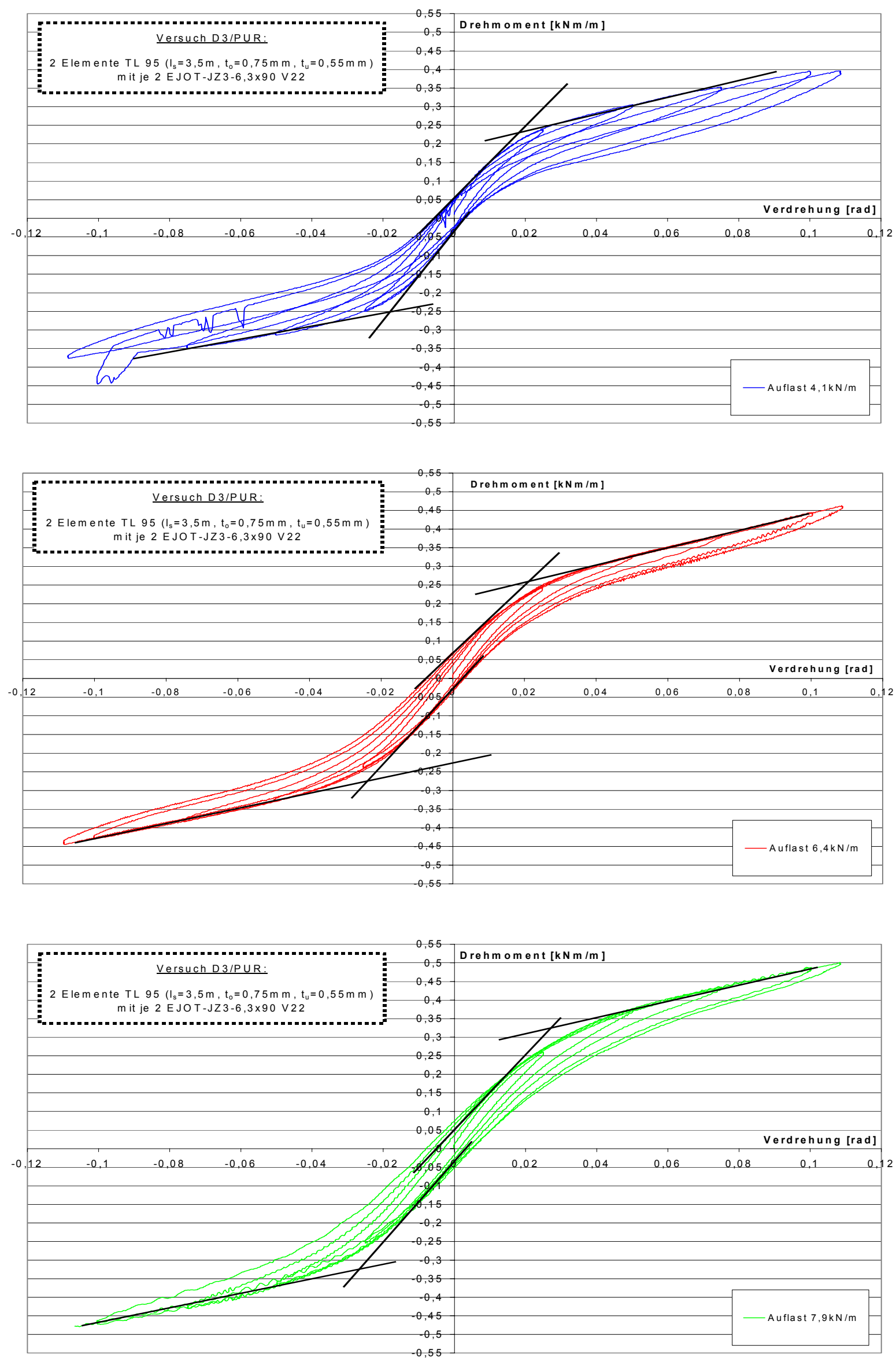

Diagramm A3: Momenten-Verdrehungsbeziehungen des Versuchs D3/PUR 

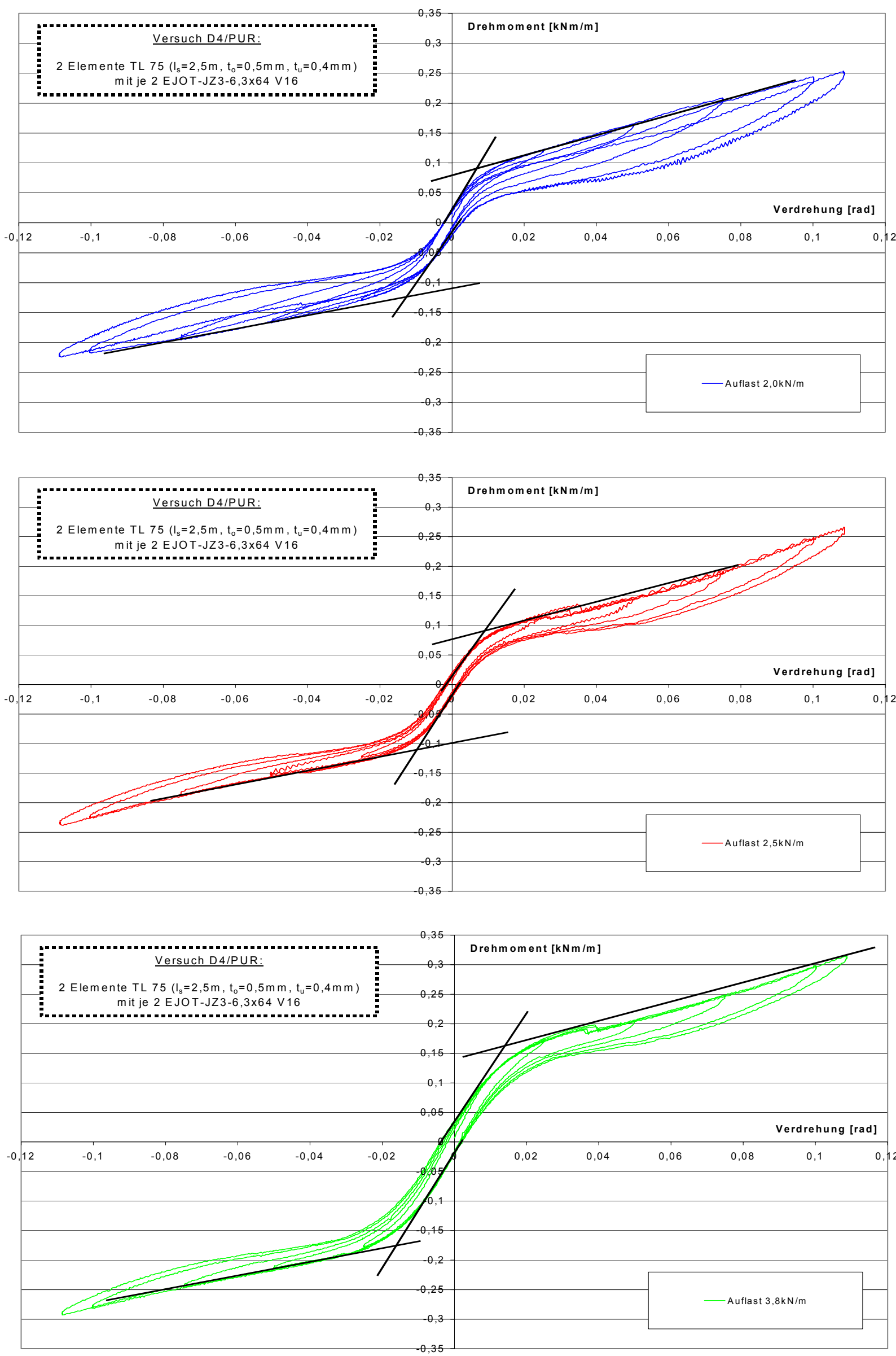

Diagramm A4: Momenten-Verdrehungsbeziehungen des Versuchs D4/PUR 

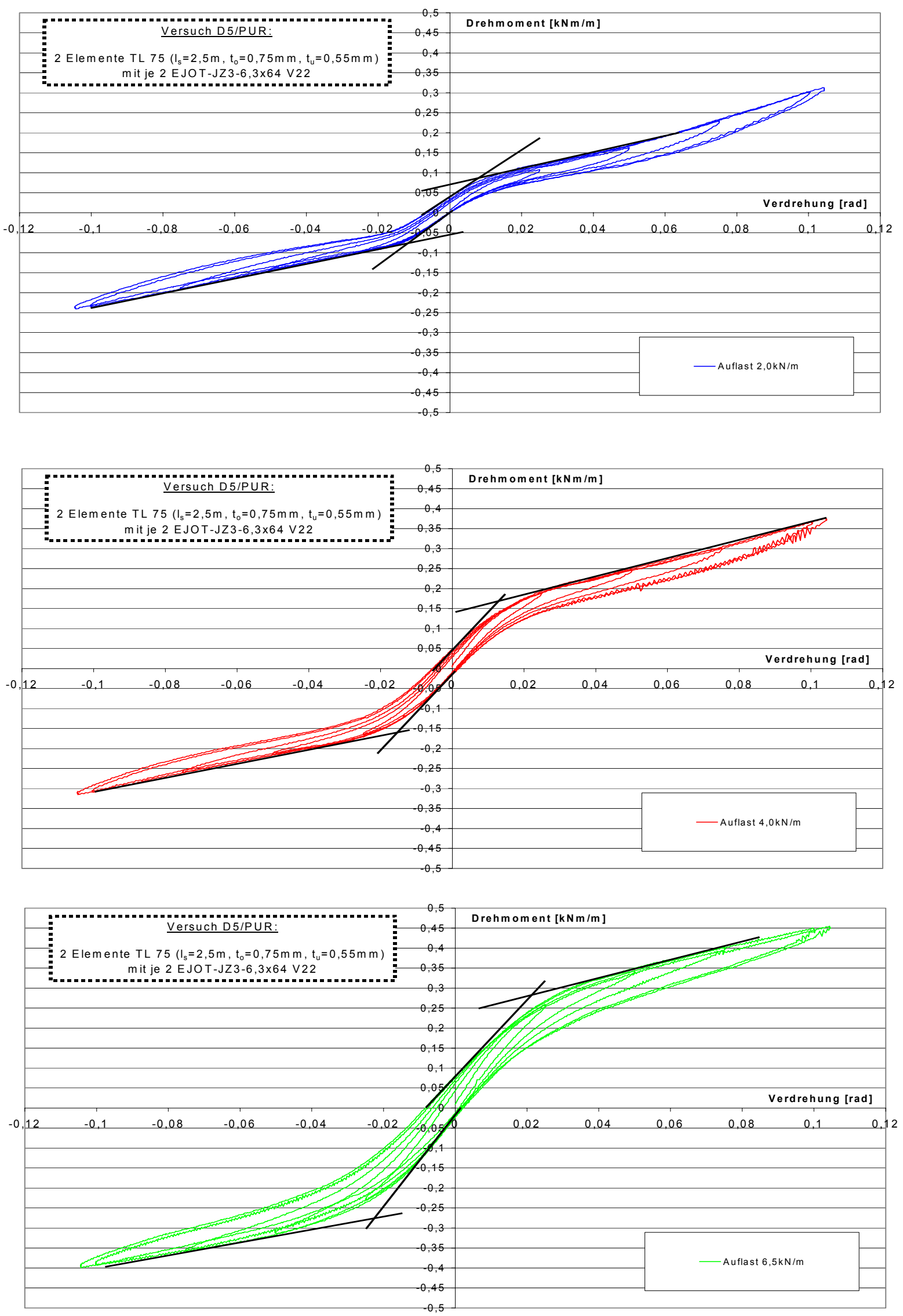

Diagramm A5: Momenten-Verdrehungsbeziehungen des Versuchs D5/PUR 

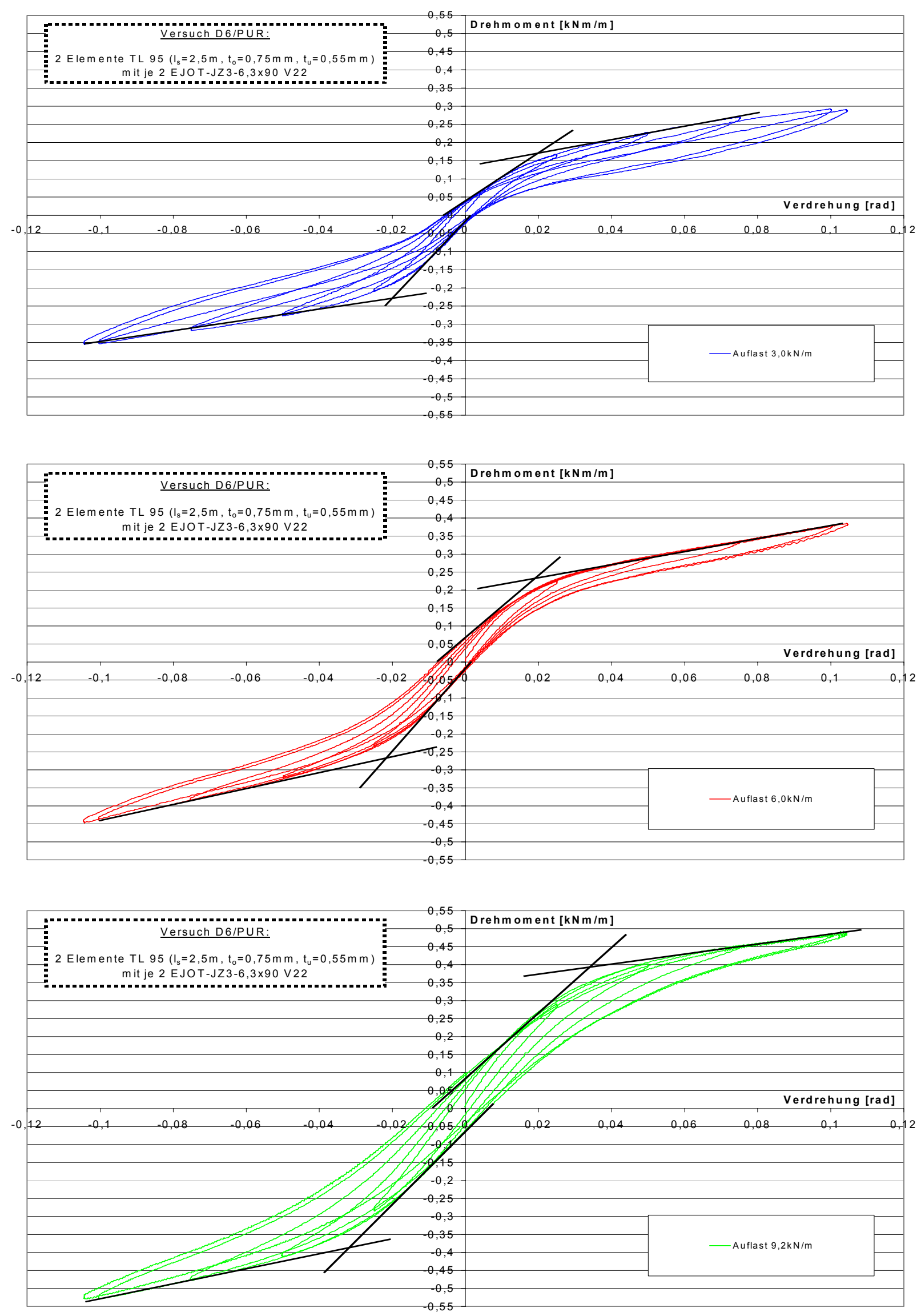

Diagramm A6: Momenten-Verdrehungsbeziehungen des Versuchs D6/PUR 

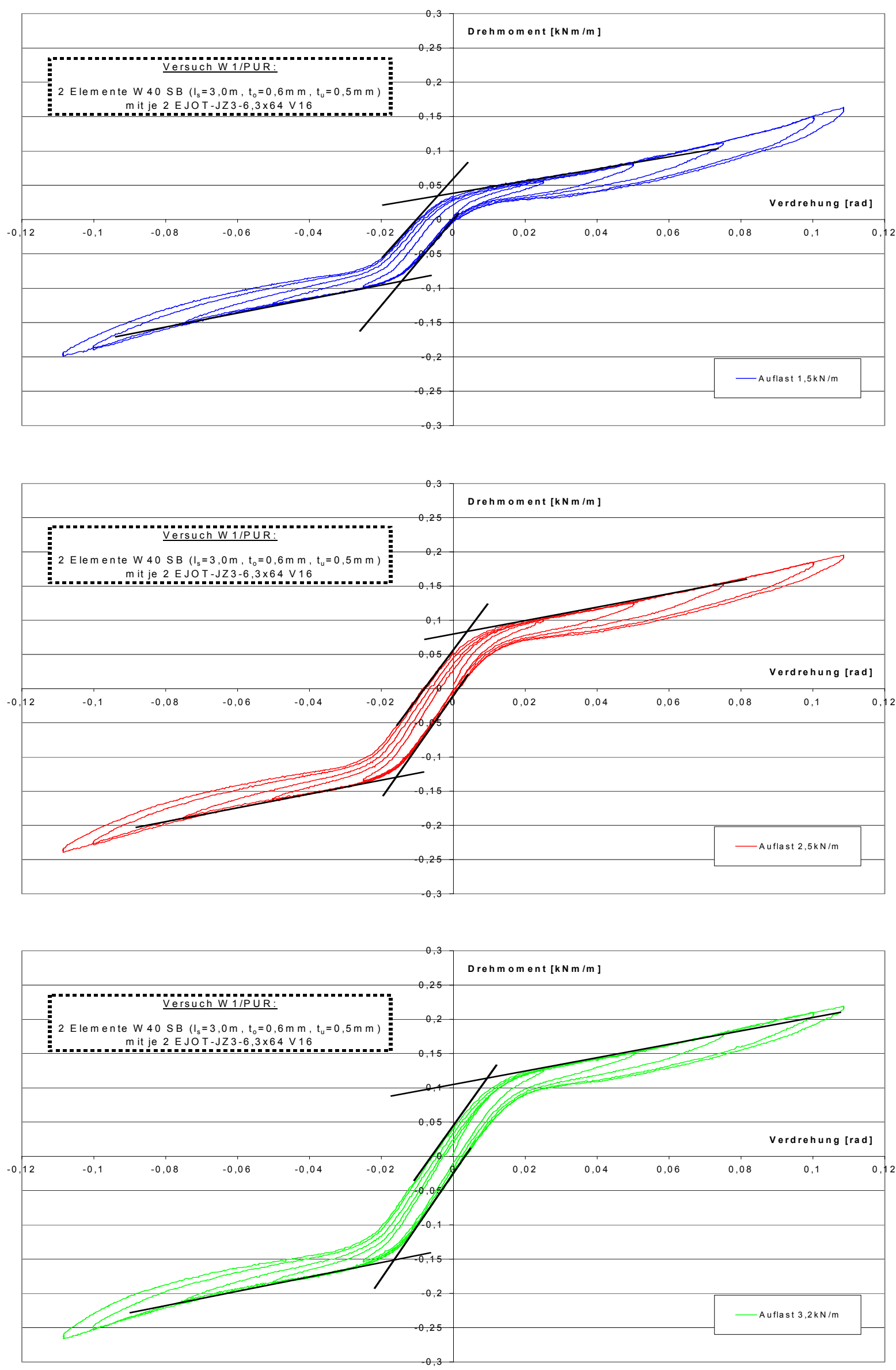

Diagramm A7: Momenten-Verdrehungsbeziehungen des Versuchs W1/PUR 

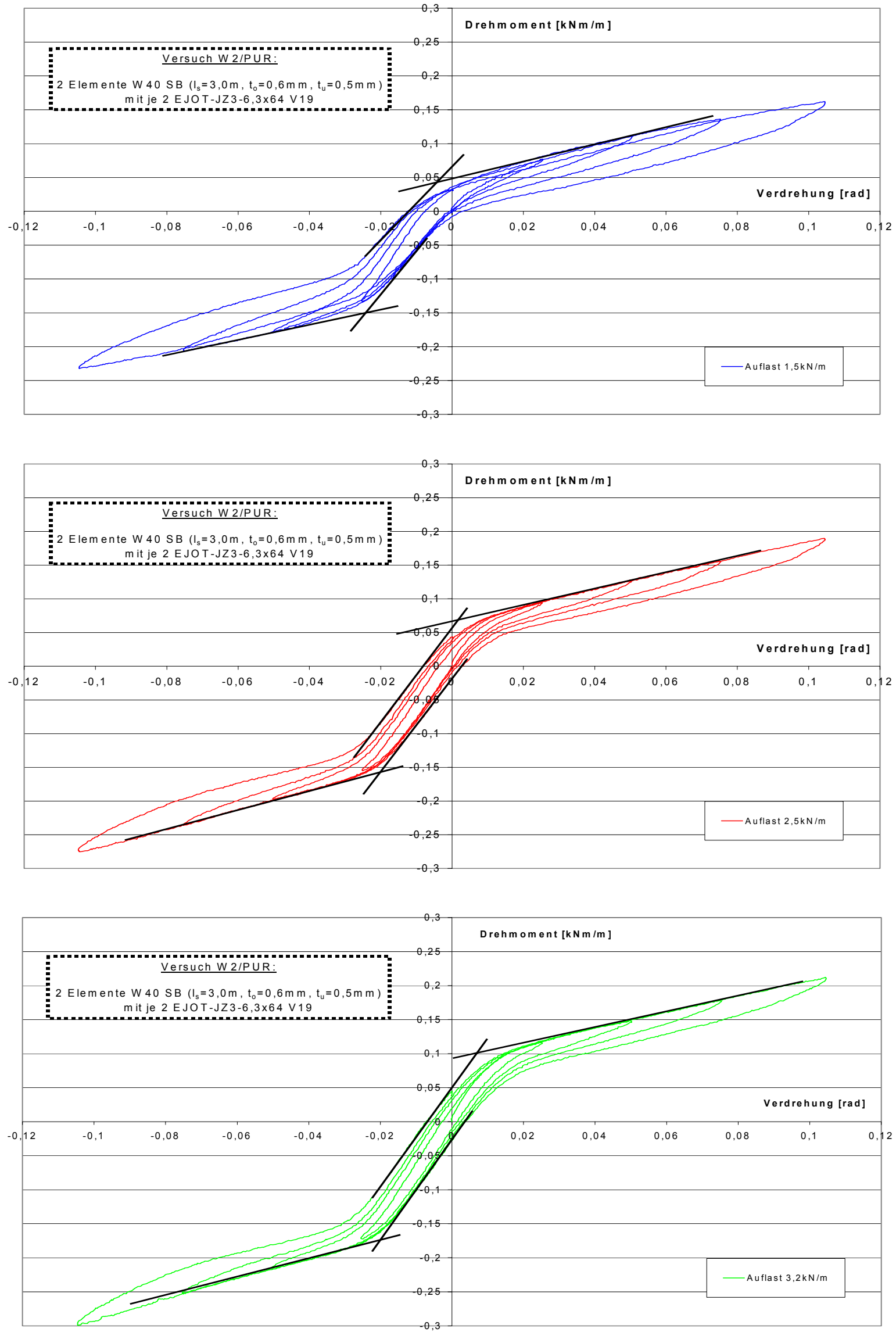

Diagramm A8: Momenten-Verdrehungsbeziehungen des Versuchs W2/PUR 

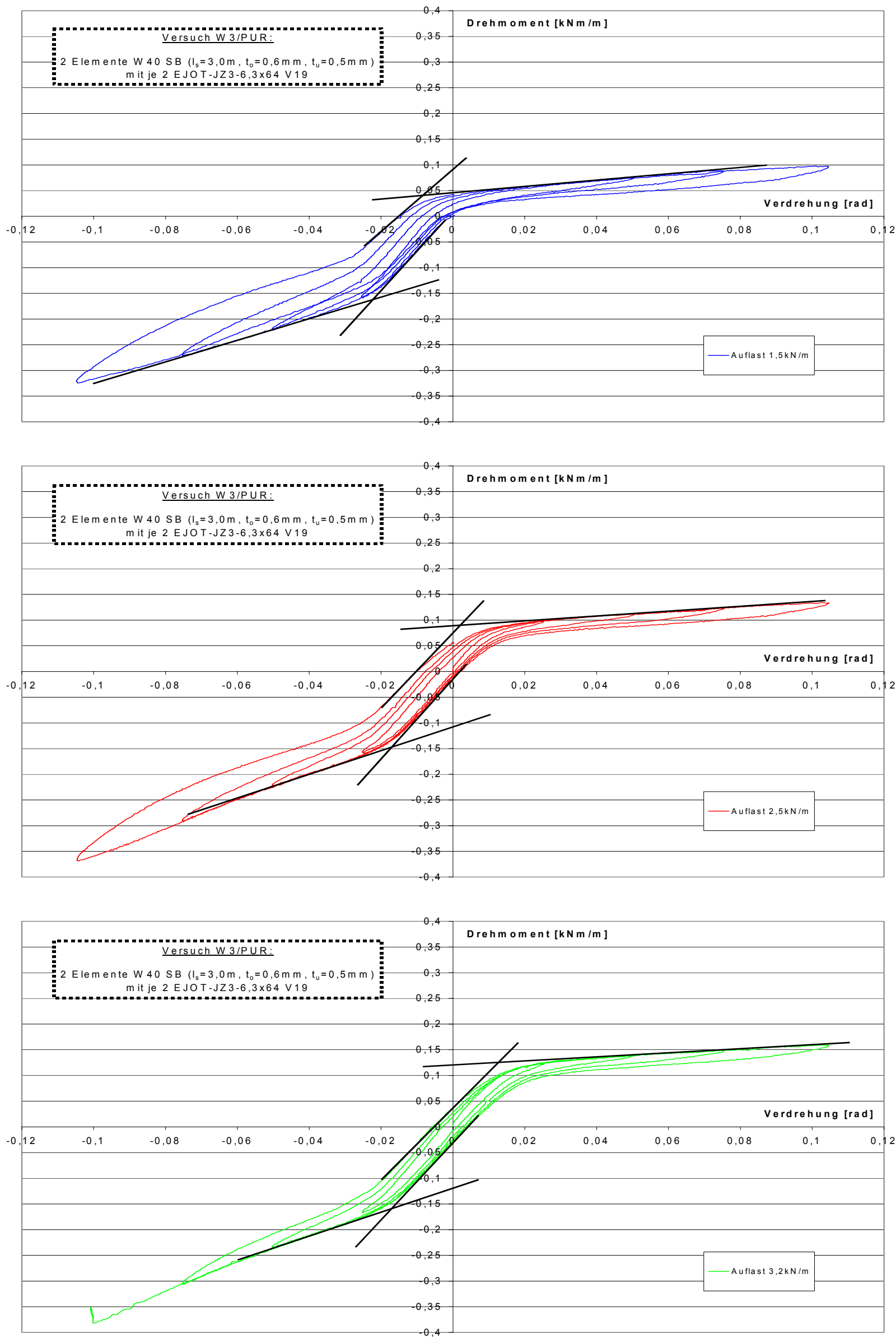

Diagramm A9: Momenten-Verdrehungsbeziehungen des Versuchs W3/PUR 

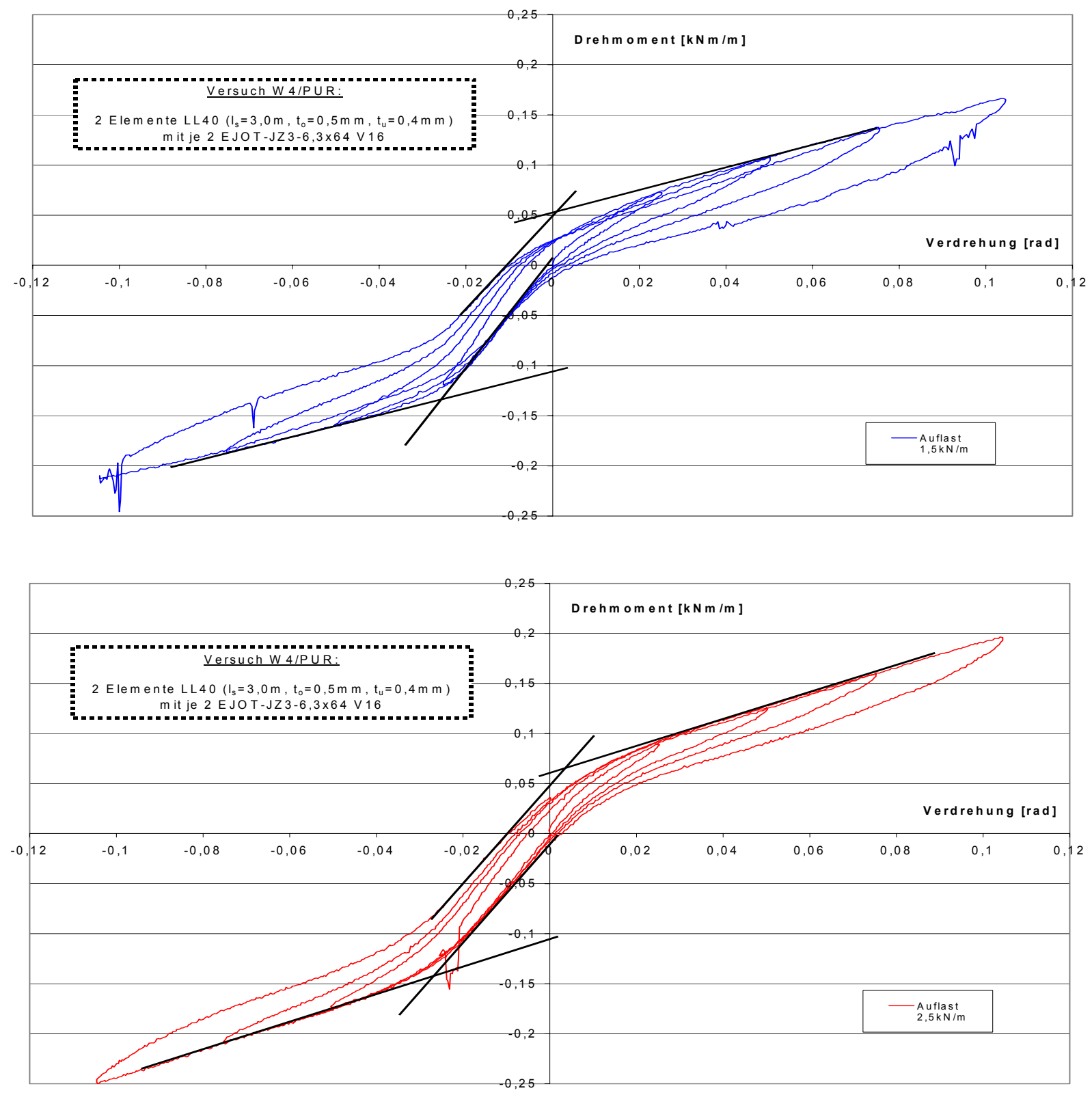

Diagramm A10: Momenten-Verdrehungsbeziehungen des Versuchs W4/PUR 

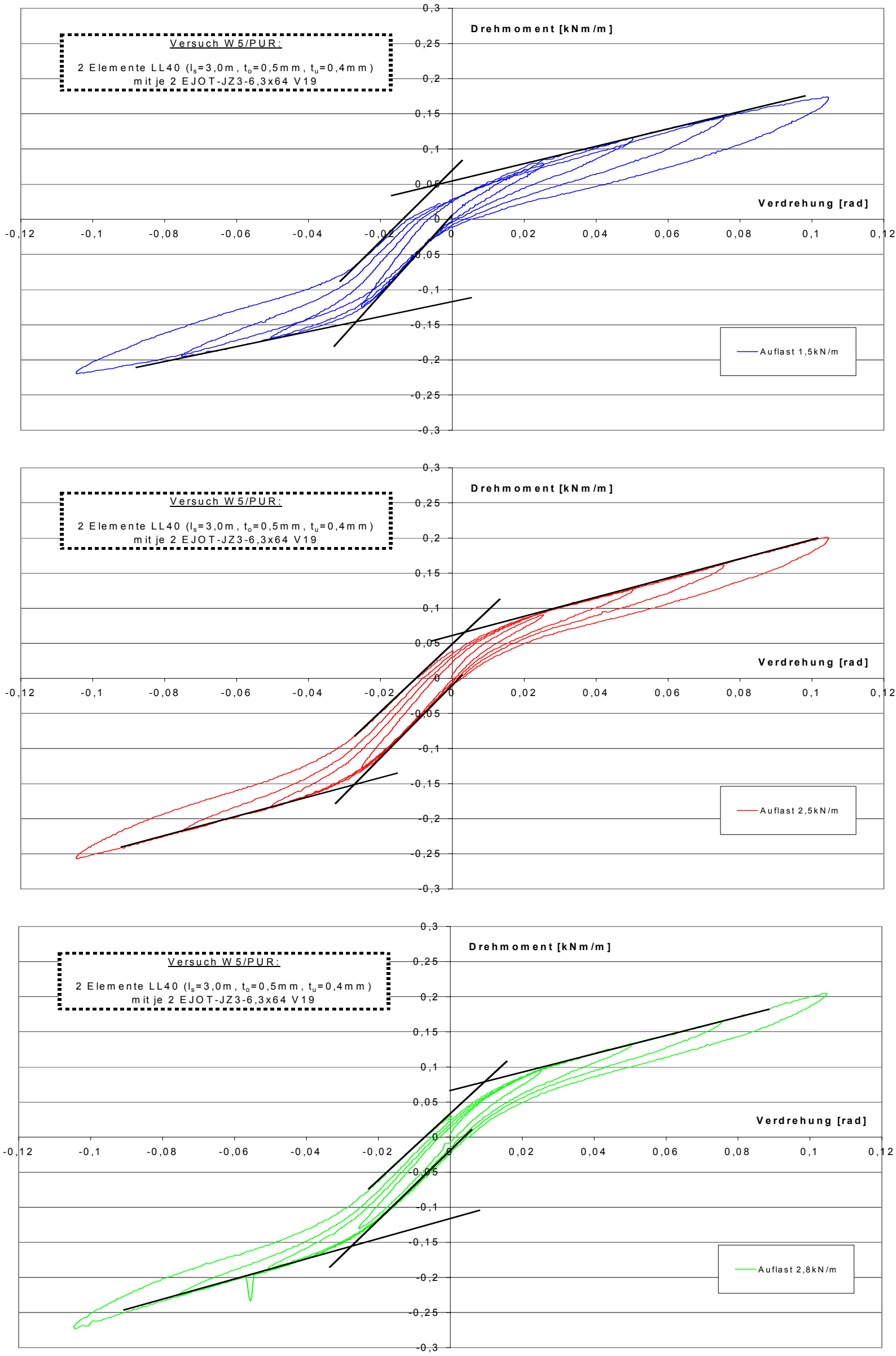

Diagramm A11: Momenten-Verdrehungsbeziehungen des Versuchs W5/PUR 

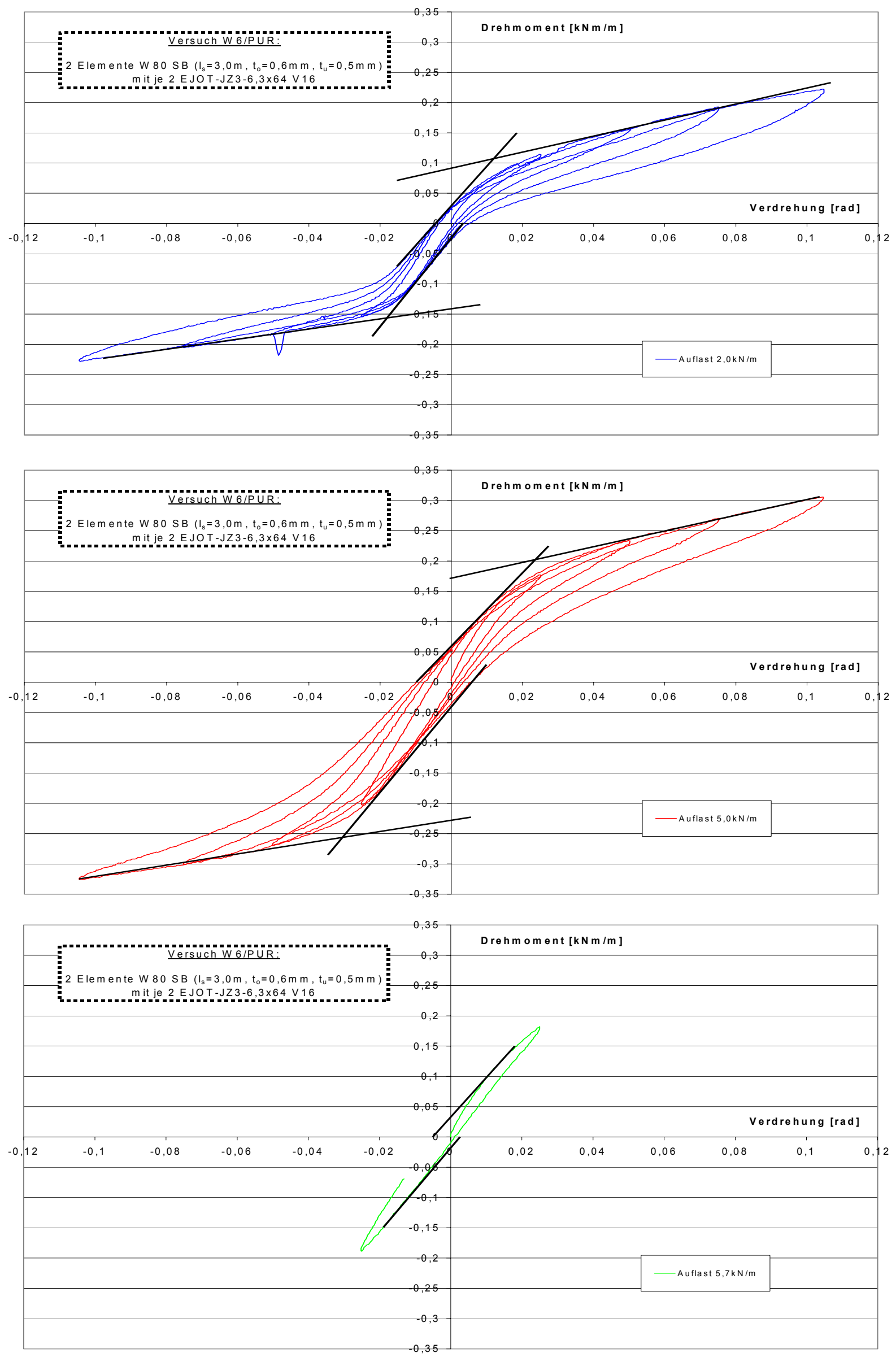

Diagramm A12: Momenten-Verdrehungsbeziehungen des Versuchs W6/PUR 

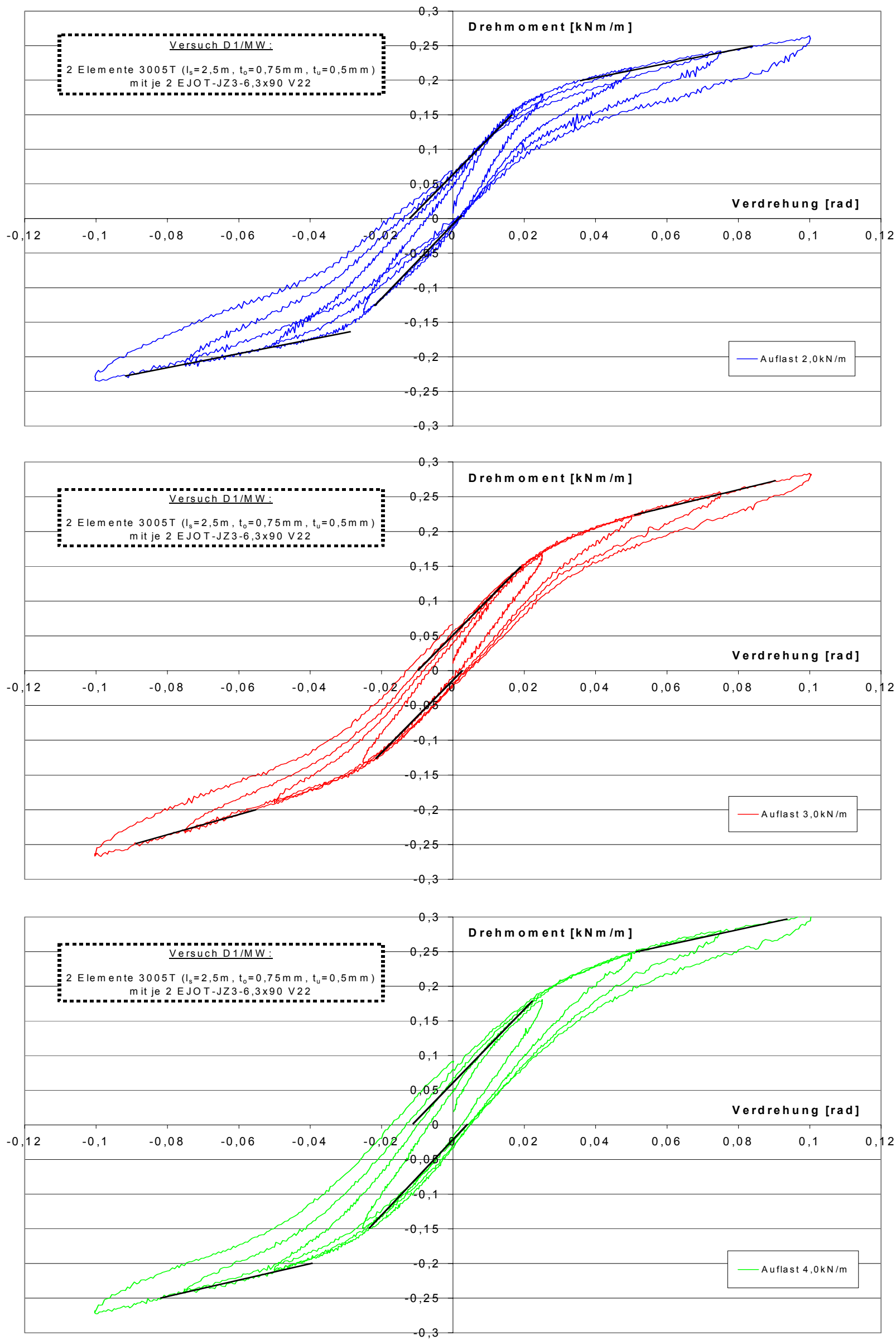

Diagramm A13: Momenten-Verdrehungsbeziehungen des Versuchs D1/MW 

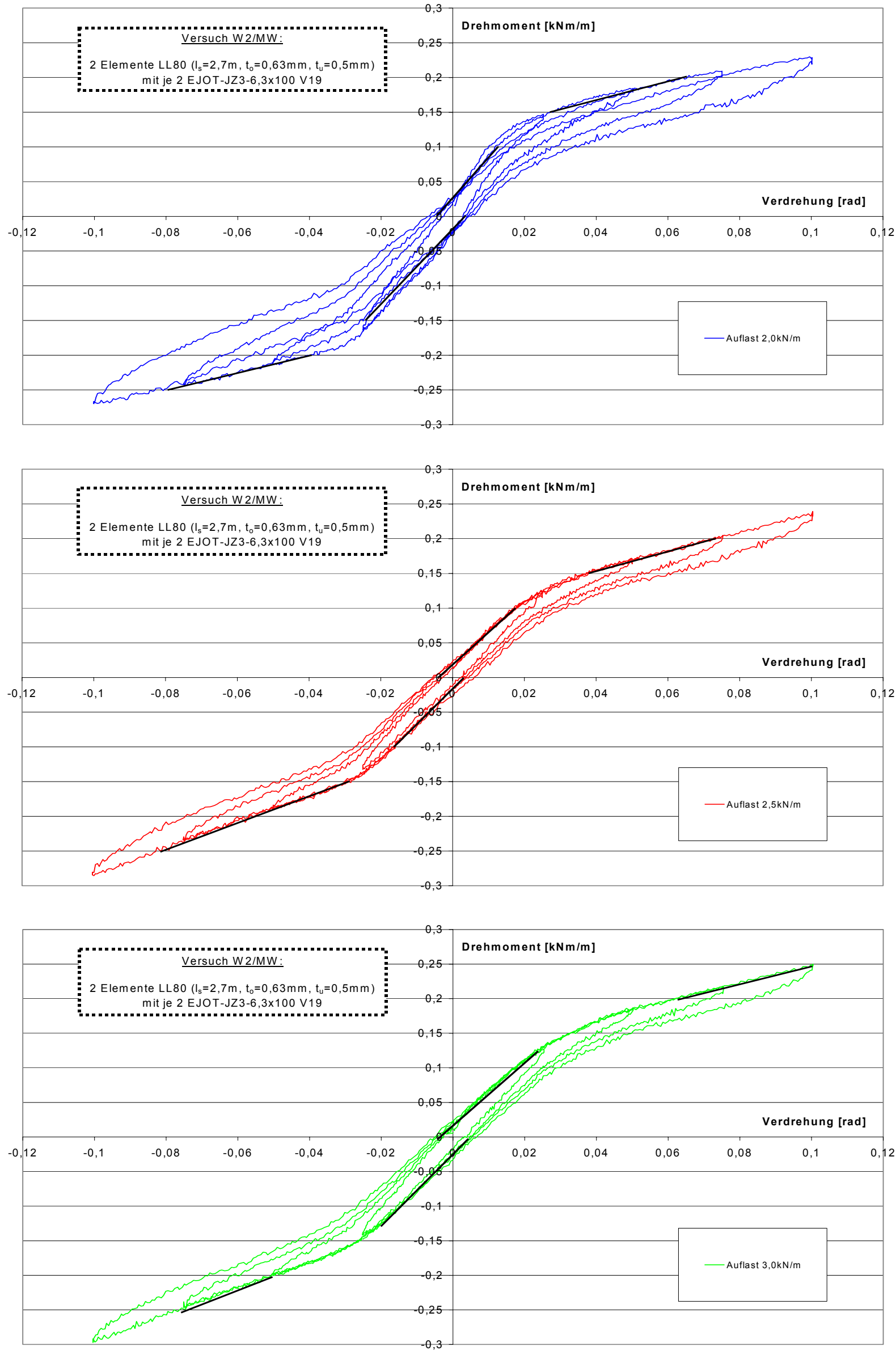

Diagramm A14: Momenten-Verdrehungsbeziehungen des Versuchs W2/MW 

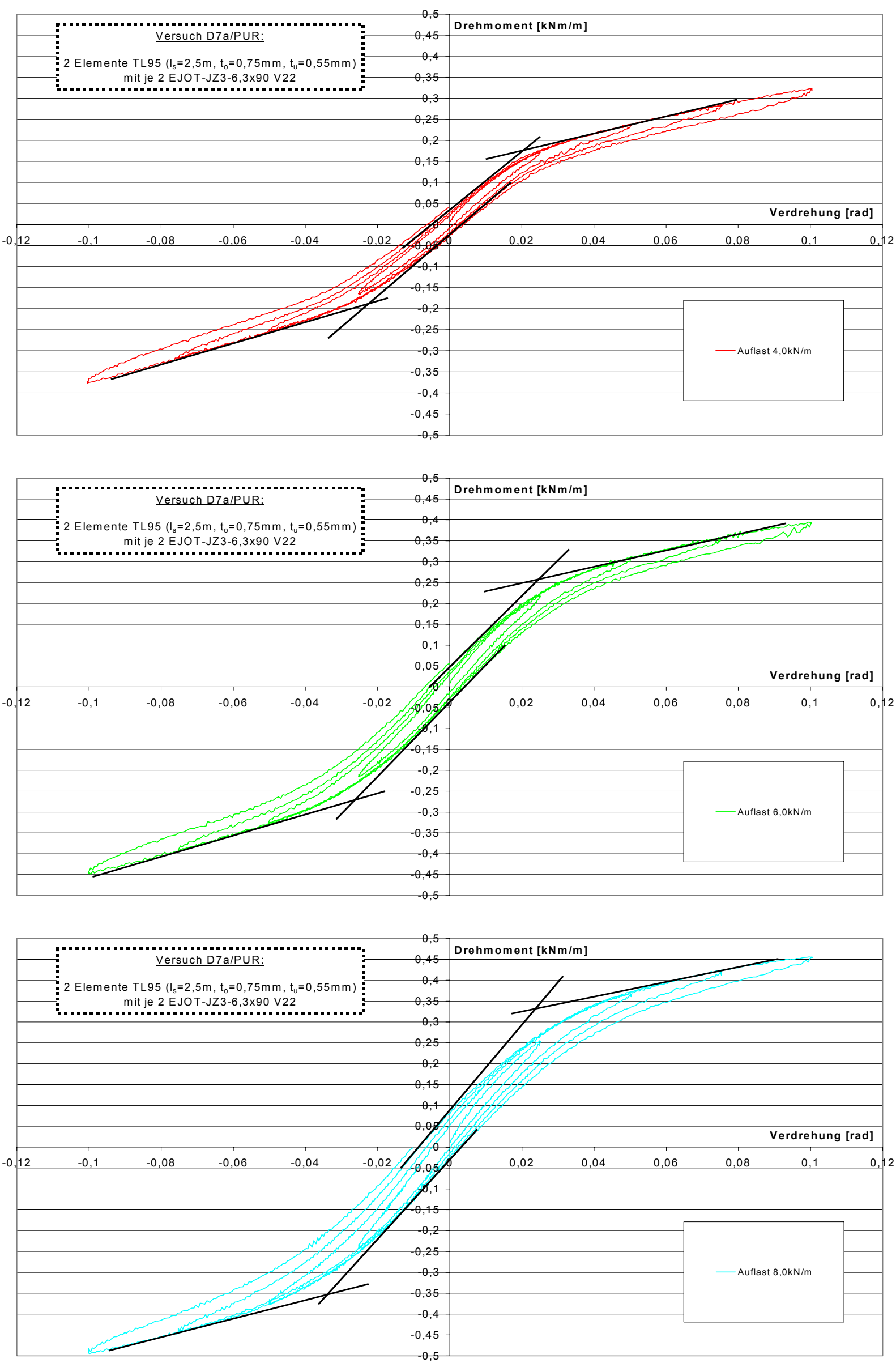

Diagramm A15: Momenten-Verdrehungsbeziehungen des Versuchs D7a/PUR 

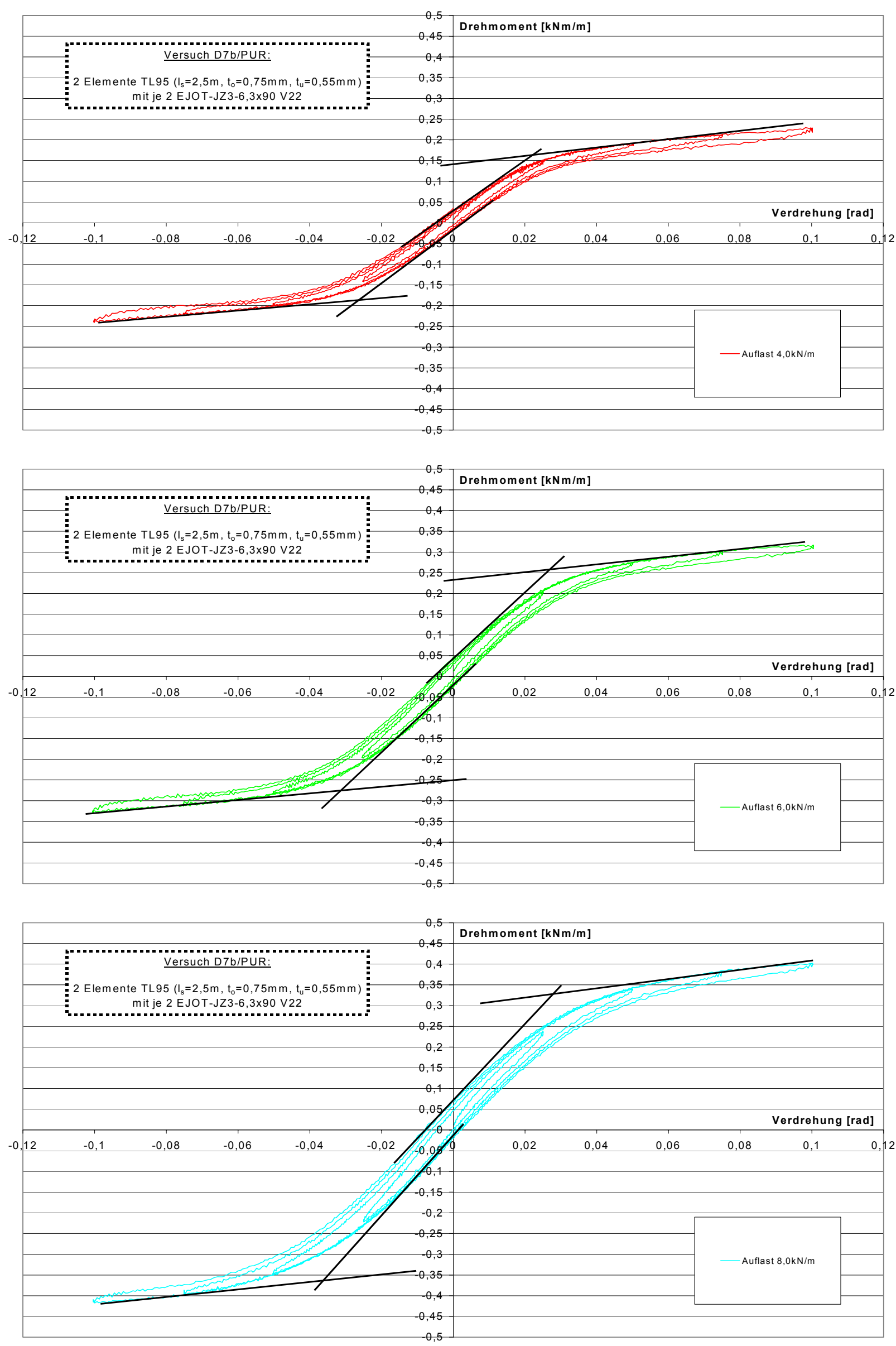

Diagramm A16: Momenten-Verdrehungsbeziehungen des Versuchs D7b/PUR 

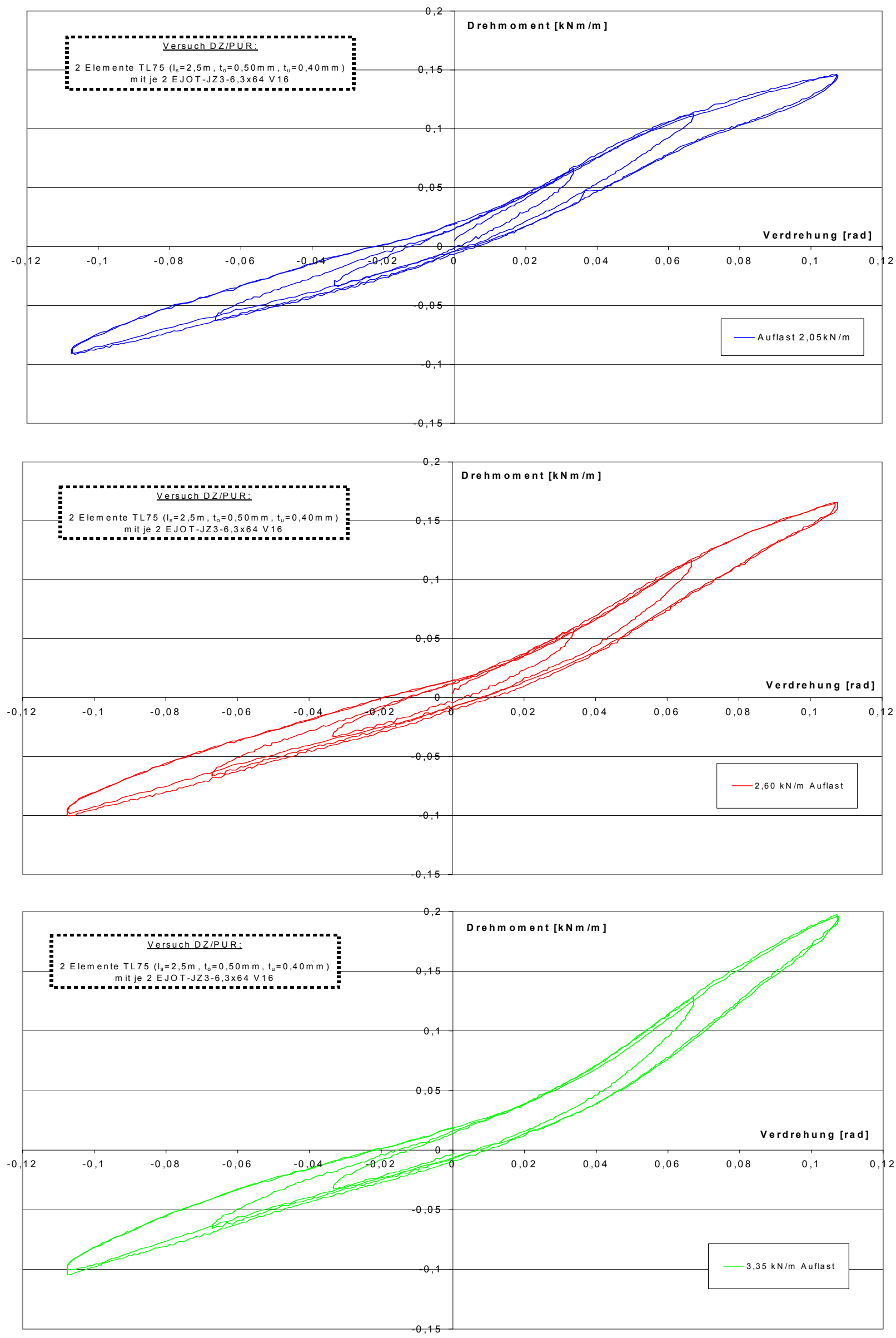

Diagramm A17: Momenten-Verdrehungsbeziehungen des Versuchs DZ/PUR 


\section{Anhang A3: Ergebnisse der Zugversuche an den Deckschichten}

\begin{tabular}{|c|c|c|c|c|c|c|c|}
\hline $\begin{array}{l}\text { Versuchs- } \\
\text { bezeich- } \\
\text { nung }\end{array}$ & Elementtyp & Deckblechtyp & $\begin{array}{c}\text { nominelle } \\
\text { Blechdicke } \\
t_{N}\end{array}$ & $\begin{array}{c}\text { Kernblech- } \\
\text { dicke } \\
t_{k}\end{array}$ & $\mathrm{R}_{\mathrm{eH}} / \mathrm{R}_{\mathrm{p} 0,2}$ & $\mathrm{R}_{\mathrm{m}}$ & $\mathrm{A}_{80 \mathrm{~mm}}$ \\
\hline & & oben/unten & {$[\mathrm{mm}]$} & {$[\mathrm{mm}]$} & {$\left[\mathrm{N} / \mathrm{mm}^{2}\right]$} & {$\left[\mathrm{N} / \mathrm{mm}^{2}\right]$} & [\%] \\
\hline \multirow{2}{*}{$\begin{array}{l}\text { D1/PUR } \\
\text { D4/PUR }\end{array}$} & \multirow{2}{*}{$\begin{array}{c}\text { HOESCH } \\
\text { Thermodach } \\
\text { TL75 }\end{array}$} & $\begin{array}{c}\text { oben: } \\
\text { trapezprofiliert }\end{array}$ & 0,50 & 0,46 & 434 & 464 & 18 \\
\hline & & $\begin{array}{l}\text { unten: } \\
\text { liniert }\end{array}$ & 0,40 & 0,49 & 439 & 508 & 23 \\
\hline \multirow{2}{*}{$\begin{array}{l}\text { D2/PUR } \\
\text { D5/PUR }\end{array}$} & \multirow{2}{*}{$\begin{array}{c}\text { HOESCH } \\
\text { Thermodach } \\
\text { TL75 }\end{array}$} & $\begin{array}{l}\text { oben: } \\
\text { trapezprofiliert }\end{array}$ & 0,75 & 0,70 & $\left.435^{*}\right)$ & 424 & 24 \\
\hline & & $\begin{array}{l}\text { unten: } \\
\text { liniert }\end{array}$ & 0,55 & 0,45 & $\left.464^{*}\right)$ & 439 & 25 \\
\hline \multirow{2}{*}{$\begin{array}{c}\text { D3/PUR } \\
\text { D6/PUR } \\
\text { D7a/PUR } \\
\text { D7b/PUR }\end{array}$} & \multirow{2}{*}{$\begin{array}{c}\text { HOESCH } \\
\text { Thermodach } \\
\text { TL95 }\end{array}$} & $\begin{array}{c}\text { oben: } \\
\text { trapezprofiliert }\end{array}$ & 0,75 & 0,68 & 401 & 496 & 23 \\
\hline & & $\begin{array}{l}\text { unten: } \\
\text { liniert }\end{array}$ & 0,55 & 0,53 & 411 & 489 & 24 \\
\hline \multirow{2}{*}{$\begin{array}{l}\text { W1/PUR } \\
\text { W2/PUR } \\
\text { W3/PUR }\end{array}$} & \multirow{2}{*}{$\begin{array}{c}\text { Salzgitter SIP } \\
\text { W40 SB }\end{array}$} & $\begin{array}{l}\text { oben: } \\
\text { liniert }\end{array}$ & 0,60 & 0,54 & $\left.424^{*}\right)$ & 415 & 30 \\
\hline & & $\begin{array}{l}\text { unten: } \\
\text { liniert }\end{array}$ & 0,50 & 0,45 & $\left.405^{*}\right)$ & 406 & 28 \\
\hline \multirow{2}{*}{$\begin{array}{l}\text { W4/PUR } \\
\text { W5/PUR }\end{array}$} & \multirow{2}{*}{$\begin{array}{l}\text { HOESCH } \\
\text { Thermowand } \\
\text { LL40 }\end{array}$} & $\begin{array}{l}\text { oben: } \\
\text { liniert }\end{array}$ & 0,50 & 0,47 & 445 & 482 & 24 \\
\hline & & $\begin{array}{l}\text { unten: } \\
\text { liniert }\end{array}$ & 0,40 & 0,37 & 377 & 458 & 29 \\
\hline \multirow[t]{2}{*}{ W6/PUR } & \multirow{2}{*}{$\begin{array}{c}\text { Salzgitter SIP } \\
\text { W80 SB }\end{array}$} & $\begin{array}{l}\text { oben: } \\
\text { liniert }\end{array}$ & 0,60 & 0,53 & 385 & 416 & 33 \\
\hline & & $\begin{array}{l}\text { unten: } \\
\text { liniert }\end{array}$ & 0,50 & 0,43 & 373 & 409 & 30 \\
\hline \multirow{2}{*}{ D1/MW } & \multirow{2}{*}{$\begin{array}{l}\text { Arcelor } \\
\text { Ondafibre } \\
3005 T\end{array}$} & $\begin{array}{c}\text { oben: } \\
\text { trapezprofiliert }\end{array}$ & 0,75 & 0,71 & 418 & 438 & 23 \\
\hline & & $\begin{array}{l}\text { unten: } \\
\text { liniert }\end{array}$ & 0,50 & 0,47 & 436 & 455 & 23 \\
\hline \multirow{2}{*}{ W1/MW } & \multirow{2}{*}{$\begin{array}{l}\text { TKHoesch } \\
\text { isorock LL80 }\end{array}$} & $\begin{array}{l}\text { oben: } \\
\text { liniert }\end{array}$ & 0,63 & 0,56 & 347 & 403 & 19 \\
\hline & & $\begin{array}{l}\text { unten: } \\
\text { liniert }\end{array}$ & 0,50 & 0,49 & 410 & 429 & 24 \\
\hline
\end{tabular}

Tabelle A3.1: ermittelte Blechdicken und mechanische Eigenschaften der Deckschichten

*) erhöhte Streckgrenze infolge Reckalterungseffekt (vgl. Abschnitt 3.1.6.2) 


\section{Anhang A4: Ergebnisse der Untersuchungen der Sandwich- Kernschichten}

\begin{tabular}{|c|c|c|c|c|c|c|c|}
\hline $\begin{array}{l}\text { Versuchs- } \\
\text { bezeich- } \\
\text { nung }\end{array}$ & Elementtyp & Dichte $\rho$ & $\begin{array}{c}\text { Zug- } \\
\text { festigkeit } \beta_{z}\end{array}$ & $\underset{E_{z}}{\text { Zugmodul }}$ & $\begin{array}{c}\text { Druck- } \\
\text { festigkeit } \beta_{D}\end{array}$ & $\begin{array}{c}\text { Druckmodul } \\
\mathrm{E}_{\mathrm{D}}\end{array}$ & $\begin{array}{l}\text { Elastizi- } \\
\text { tätsmo- } \\
\text { dul } E_{S}\end{array}$ \\
\hline & & {$\left[\mathrm{kg} / \mathrm{m}^{3}\right]$} & {$\left[\mathrm{N} / \mathrm{mm}^{2}\right]$} & {$\left[\mathrm{N} / \mathrm{mm}^{2}\right]$} & {$\left[\mathrm{N} / \mathrm{mm}^{2}\right]$} & {$\left[\mathrm{N} / \mathrm{mm}^{2}\right]$} & {$\left[\mathrm{N} / \mathrm{mm}^{2}\right]$} \\
\hline \multirow{3}{*}{$\begin{array}{l}\text { D1/PUR } \\
\text { D4/PUR }\end{array}$} & $\mathrm{HOESCH}$ & 39,8 & 0,08 & 4,4 & 0,14 & 3,4 & 3,9 \\
\hline & Thermodach & 40,5 & 0,10 & 4,6 & 0,16 & 3,3 & 4,0 \\
\hline & & 39,9 & 0,10 & 4,2 & 0,14 & 3,5 & 3,9 \\
\hline \multirow{3}{*}{$\begin{array}{l}\text { D2/PUR } \\
\text { D5/PUR }\end{array}$} & HOESCH & 40,2 & 0,10 & 5,0 & 0,14 & 4,0 & 4,5 \\
\hline & Thermodach & 40,6 & 0,10 & 5,2 & 0,15 & 4,0 & 4,6 \\
\hline & TL75 & 40,4 & 0,11 & 5,0 & 0,13 & 4,0 & 4,5 \\
\hline \multirow{3}{*}{$\begin{array}{c}\text { D3/PUR } \\
\text { D6/PUR } \\
\text { D7a/PUR } \\
\text { D7b/PUR }\end{array}$} & HOESCH & 38,5 & 0,08 & 4,4 & 0,13 & 3,6 & 4.0 \\
\hline & Thermodach & 38,8 & 0,09 & 4,0 & 0,11 & 3,6 & 3,8 \\
\hline & TL95 & 38,2 & 0,10 & 4,2 & 0,15 & 3,4 & 3,8 \\
\hline \multirow{2}{*}{$\begin{array}{l}\text { W1/PUR } \\
\text { W2/PUR } \\
\text { W3/PUR }\end{array}$} & Salzgitter SIP & 43,0 & 0,08 & 4,5 & 0,13 & 3,8 & 4,2 \\
\hline & W40 SB & $\begin{array}{l}42,7 \\
12,8\end{array}$ & $\begin{array}{l}0,08 \\
0,08\end{array}$ & 4,8 & 0,13 & 3,0 & 3,9 \\
\hline \multirow{3}{*}{$\begin{array}{l}\text { W4/PUR } \\
\text { W5/PUR }\end{array}$} & $\mathrm{HOESCH}$ & 39,2 & 0,09 & 4,0 & 0,17 & 2,7 & 3,4 \\
\hline & Thermowand & 39,5 & 0,08 & 4,2 & 0,17 & 3,4 & 3,8 \\
\hline & LL40 & 38,8 & 0,08 & 3,9 & 0,15 & 2,9 & 3,4 \\
\hline \multirow{3}{*}{ W6/PUR } & Salzaitter SIP & 42,6 & 0,10 & 4,8 & 0,12 & 3,0 & 3,9 \\
\hline & W80 SB & 42,5 & 0,09 & 5,2 & 0,14 & 2,8 & 4,0 \\
\hline & & & & & & & \\
\hline \multirow{2}{*}{ D1/MW } & Arcelor Ondafi- & $\begin{array}{l}115 \\
119\end{array}$ & 0,06 & 9,9 & 0,09 & 7,8 & 8,9 \\
\hline & bre 3005T & 117 & 0,05 & 9,9 & 0,10 & 7,9 & 8,9 \\
\hline \multirow{3}{*}{ W2/MW } & \multirow{3}{*}{$\begin{array}{l}\text { TKHoesch iso- } \\
\text { rock LL80 }\end{array}$} & 110 & 0,04 & 10,4 & 0,06 & 3,8 & 7,1 \\
\hline & & 115 & 0,05 & 8,5 & 0,08 & 4,2 & 6,4 \\
\hline & & 108 & 0,06 & 9,8 & 0,08 & 4,4 & 7,1 \\
\hline
\end{tabular}

Tabelle A4.1: mechanische Eigenschaften der Sandwich-Kernschichten 


\section{Anhang A5: statistische Auswertungen}

\begin{tabular}{|c|c|c|c|c|c|c|c|c|}
\hline \multirow{2}{*}{$\begin{array}{l}\text { Versuchs- } \\
\text { bezeich- } \\
\text { nung }\end{array}$} & $\begin{array}{c}E_{s} \\
\text { (Mittelwert) }\end{array}$ & $c_{\vartheta 1} \ldots$ pos & $c_{91}{ }^{n e g}$ & $x_{1}=\frac{c_{\vartheta 1} \_p o s}{E_{S}}$ & $x_{2}=\frac{c_{\vartheta 1}{ }^{n e g}}{E_{S}}$ & $\frac{x_{1}}{\bar{x}}$ & $\frac{x_{2}}{\bar{x}}$ & \\
\hline & {$\left[\mathrm{N} / \mathrm{mm}^{2}\right]$} & {$[\mathrm{kNm} / \mathrm{m}]$} & {$[\mathrm{kNm} / \mathrm{m}]$} & {$\left[1000 \mathrm{~mm}^{2}\right]$} & {$\left[1000 \mathrm{~mm}^{2}\right]$} & {$[-]$} & {$[-]$} & \\
\hline \multirow{3}{*}{ D1/PUR } & \multirow{3}{*}{3,9} & 6,17 & 6,54 & 1,58 & 1,68 & 0,72 & 0,77 & \\
\hline & & 9,14 & 9,22 & 2,34 & 2,36 & 1,07 & 1,08 & \\
\hline & & 8,70 & 9,17 & 2,23 & 2,35 & 1,02 & 1,08 & \\
\hline \multirow{3}{*}{ D2/PUR } & \multirow{3}{*}{4,5} & 8,33 & 11,81 & 1,85 & 2,62 & 0,85 & 1,20 & \\
\hline & & 8,88 & 10,70 & 1,97 & 2,38 & 0,90 & 1,09 & \\
\hline & & 8,77 & 10,42 & 1,95 & 2,31 & 0,89 & 1,06 & \\
\hline \multirow{3}{*}{ D3/PUR } & \multirow{3}{*}{3,9} & 9,66 & 11,43 & 2,48 & 2,93 & 1,13 & 1,34 & \\
\hline & & 6,02 & 6,76 & 1,54 & 1,73 & 0,71 & 0,79 & \\
\hline & & 10,20 & 10,08 & 2,62 & 2,59 & 1,20 & 1,18 & \\
\hline \multirow{3}{*}{ D4/PUR } & \multirow{3}{*}{3,9} & 9,17 & 8,47 & 2,35 & 2,17 & 1,08 & 0,99 & \\
\hline & & 8,47 & 8,65 & 2,17 & 2,22 & 0,99 & 1,01 & \\
\hline & & 9,50 & 9,90 & 2,44 & 2,54 & 1,11 & 1,16 & \\
\hline \multirow{3}{*}{ D5/PUR } & \multirow{3}{*}{4,5} & 5,90 & 6,30 & 1,31 & 1,40 & 0,60 & 0,64 & \\
\hline & & 8,50 & 9,60 & 1,89 & 2,13 & 0,86 & 0,98 & \\
\hline & & 9,50 & 10,90 & 2,11 & 2,42 & 0,97 & 1,11 & \\
\hline \multirow{3}{*}{ D6/PUR } & \multirow{3}{*}{3,9} & 6,70 & 9,10 & 1,72 & 2,33 & 0,78 & 1,07 & \\
\hline & & 8,50 & 10,60 & 2,18 & 2,72 & 1,00 & 1,24 & \\
\hline & & 9,10 & 10,20 & 2,33 & 2,62 & 1,07 & 1,20 & \\
\hline \multirow{4}{*}{ D7a/PUR } & \multirow{3}{*}{3,9} & 7,17 & 7,50 & 1,84 & 1,92 & 0,84 & 0,88 & \\
\hline & & 8,85 & 8,95 & 2,27 & 2,29 & 1,04 & 1,05 & \\
\hline & & 9,49 & 9,89 & 2,43 & 2,54 & 1,11 & 1,16 & \\
\hline & & \multicolumn{2}{|c|}{ Mittelwert $\bar{x}$} & \multicolumn{2}{|c|}{$\underline{2,19}$} & \multicolumn{2}{|c|}{$\underline{5 \%-F r a k t i l w e r t}$} & $1,62 \times E_{\underline{s}}$ \\
\hline \multirow{3}{*}{ W1/PUR } & \multirow{3}{*}{4,1} & 5,88 & 6,12 & 1,43 & 1,49 & 0,88 & 0,91 & \\
\hline & & 6,93 & 7,37 & 1,69 & 1,80 & 1,03 & 1,10 & \\
\hline & & 7,20 & 7,69 & 1,76 & 1,87 & 1,08 & 1,15 & \\
\hline \multirow{3}{*}{ W2/PUR } & \multirow{3}{*}{4,1} & 5,32 & 6,17 & 1,30 & 1,51 & 0,79 & 0,92 & \\
\hline & & 6,97 & 6,94 & 1,70 & 1,69 & 1,04 & 1,04 & \\
\hline & & 7,43 & 7,43 & 1,81 & 1,81 & 1,11 & 1,11 & \\
\hline & & 6,46 & 7,75 & 1,58 & 1,89 & 0,96 & 1,16 & \\
\hline W3/PUR & 4,1 & 7,41 & 7,68 & 1,81 & 1,87 & 1,11 & 1,15 & \\
\hline & & 7,46 & 7,72 & 1,82 & 1,88 & 1,11 & 1,15 & \\
\hline W4/PUR & 35 & 4,89 & 5,29 & 1,40 & 1,51 & 0,85 & 0,93 & \\
\hline VV4/PUR & 3,5 & 4,90 & 5,08 & 1,40 & 1,45 & 0,86 & 0,89 & \\
\hline & & 4,94 & 5,22 & 1,53 & 1,59 & 0,94 & 0,98 & \\
\hline W5/PUR & 3,5 & 4,90 & 5,13 & 1,41 & 1,49 & 0,86 & 0,91 & \\
\hline & & 6,46 & 7,06 & 1,40 & 1,47 & 0,86 & 0,90 & \\
\hline & & 6,10 & 7,63 & 1,61 & 1,76 & 0,99 & 1,08 & \\
\hline W6/PUR & 4,0 & 6,51 & 6,91 & 1,53 & 1,91 & 0,93 & 1,17 & \\
\hline & & 5,88 & 6,12 & 1,63 & 1,73 & 1,00 & 1,06 & \\
\hline & & Mittel & ert $\bar{x}$ & & & $5 \%-\mathrm{Fr}$ & ilwert & $\underline{1,21 \times E_{s}}$ \\
\hline & & & & Anzahl & Verte $n=$ & & & \\
\hline & & & & Variations & effizient s= & & & \\
\hline & & & & & & & & \\
\hline
\end{tabular}




\begin{tabular}{|c|c|c|c|c|c|c|c|c|c|}
\hline \multirow{2}{*}{$\begin{array}{l}\text { Ver- } \\
\text { suchs- } \\
\text { bezeich- } \\
\text { nung }\end{array}$} & $\begin{array}{c}E_{s} \\
\text { (Mittel- } \\
\text { wert) }\end{array}$ & $t_{\text {Koben }}$ & $c_{92} p o s$ & $c_{92 \_} n e g$ & $x_{1}=\frac{c_{92}-p o s}{E_{S} \cdot t_{K}}$ & $x_{2}=\frac{c_{92} \_n e g}{E_{S} \cdot t_{K}}$ & $\frac{x_{1}}{\bar{x}}$ & $\frac{x_{2}}{\bar{x}}$ & \\
\hline & {$\left[\mathrm{N} / \mathrm{mm}^{2}\right]$} & {$[\mathrm{mm}]$} & {$[\mathrm{kNm} / \mathrm{m}]$} & {$[\mathrm{kNm} / \mathrm{m}]$} & {$\left[1000 \mathrm{~mm}^{2}\right]$} & {$\left[1000 \mathrm{~mm}^{2}\right]$} & {$[-]$} & {$[-]$} & \\
\hline \multirow{3}{*}{ D1/PUR } & \multirow{3}{*}{3,9} & \multirow{3}{*}{0,46} & 1,57 & 1,57 & 0,87 & 0,87 & 1,21 & 1,21 & \\
\hline & & & 1,41 & 1,33 & 0,79 & 0,74 & 1,9 & 1,03 & \\
\hline & & & 1,43 & 1,35 & 0,80 & 0,75 & 1,10 & 1,04 & \\
\hline \multirow{3}{*}{ D2/PUR } & \multirow{3}{*}{4,5} & \multirow{3}{*}{0,70} & 1,87 & 1,58 & 0,59 & 0,50 & 0,82 & 0,69 & \\
\hline & & & 2,04 & 1,93 & 0,65 & 0,61 & 0,90 & 0,85 & \\
\hline & & & 2,00 & 1,79 & 0,63 & 0,57 & 0,88 & 0,79 & \\
\hline \multirow{3}{*}{ D3/PUR } & \multirow{3}{*}{3,9} & \multirow{3}{*}{0,68} & 2,18 & 2,62 & 0,82 & 0,99 & 1,14 & 1,00 & \\
\hline & & & 2,30 & 1,90 & 0,88 & 0,73 & 1,22 & 1,02 & \\
\hline & & & 2,20 & 2,00 & 0,83 & 0,74 & 1,15 & 1,09 & \\
\hline \multirow{3}{*}{ D4/PUR } & \multirow{3}{*}{3,9} & \multirow{3}{*}{0,46} & 1,64 & 1,17 & 0,91 & 0,65 & 1,26 & 0,90 & \\
\hline & & & 1,70 & 1,20 & 0,93 & 0,68 & 1,29 & 0,94 & \\
\hline & & & 1,65 & 1,18 & 0,92 & 0,66 & 1,27 & 0,91 & \\
\hline \multirow{3}{*}{ D5/PUR } & \multirow{3}{*}{4,5} & \multirow{3}{*}{0,70} & 2,05 & 1,75 & 0,65 & 0,56 & 0,90 & 0,77 & \\
\hline & & & 2,17 & 1,79 & 0,69 & 0,57 & 0,95 & 0,79 & \\
\hline & & & 2,22 & 1,59 & 0,70 & 0,50 & 0,98 & 0,70 & \\
\hline \multirow{4}{*}{ D6/PUR } & \multirow{4}{*}{3,9} & \multirow{3}{*}{0,68} & 2,08 & 1,53 & 0,79 & 0,58 & 1,09 & 0,80 & \\
\hline & & & 1,79 & 2,27 & 0,68 & 0,86 & 0,93 & 1,18 & \\
\hline & & & 1,40 & 2,13 & 0,53 & 0,80 & 0,73 & 1,11 & \\
\hline & & & \multicolumn{2}{|c|}{ Mittelwert $\bar{x}$} & \multicolumn{2}{|c|}{$\underline{0,72}$} & \multicolumn{2}{|c|}{ 5\%-Fraktilwert } & $\underline{0,44 \times E_{s}} \underline{x t}$ \\
\hline \multirow{3}{*}{ W1/PUR } & \multirow{3}{*}{4,1} & \multirow{3}{*}{0,54} & 0,84 & 1,06 & 0,38 & 0,48 & 0,61 & 0,78 & \\
\hline & & & 0,99 & 1,02 & 0,45 & 0,46 & 0,73 & 0,75 & \\
\hline & & & 0,96 & 0,95 & 0,44 & 0,43 & 0,71 & 0,70 & \\
\hline & & & 1,26 & 1,14 & 0,57 & 0,51 & 0,93 & 0,83 & \\
\hline W2/PUR & 4,1 & 0,54 & 1,30 & 1,43 & 0,59 & 0,65 & 0,95 & 1,05 & \\
\hline & & & 1,16 & 1,36 & 0,53 & 0,61 & 0,85 & 0,99 & \\
\hline W4/PUR & 35 & 0.47 & 1,17 & 1,06 & 0,71 & 0,65 & 1,16 & 1,05 & \\
\hline אוש ולה & , & ו וד, & 1,40 & 1,40 & 0,85 & 0,85 & 1,37 & 1,38 & \\
\hline & & & 1,23 & 1,09 & 0,75 & 0,66 & 1,21 & 1,08 & \\
\hline W5/PUR & 3,5 & 0,47 & 1,36 & 1,41 & 0,83 & 0,86 & 1,34 & 1,39 & \\
\hline & & & 1,28 & 1,47 & 0,78 & 0,89 & 1,26 & 1,45 & \\
\hline W6/PIR & 40 & 053 & 1,31 & 0,95 & 0,61 & 0,45 & 1,00 & 0,73 & \\
\hline הוש & 4,0 & , & 1,32 & 0,94 & 0,62 & 0,44 & 1,01 & 0,72 & \\
\hline & & & Mittelv & ert $\bar{x}$ & $\underline{0,}$ & & $\underline{5 \%-\mathrm{Fr}}$ & tilwert & $\underline{0,38 \times E_{s} \times t}$ \\
\hline & & & & & Anzahl V & lerte $n=$ & & & \\
\hline & & & & & Variationskc & effizient s= & & & \\
\hline & & & & & $\mathrm{k}_{\mathrm{s}}$ & & & & \\
\hline
\end{tabular}

Tabelle A5.2: statistische Auswertung der Werte $c_{92}$ der Elemente mit Polyurethan-Kernschicht 


\begin{tabular}{|c|c|c|c|c|c|c|c|c|}
\hline \multirow[t]{2}{*}{$\begin{array}{c}\text { Versuchs- } \\
\text { bezeichnung }\end{array}$} & $\mathrm{E}_{\mathrm{s}}$ (Mittelwert) & $c_{91}$ pos & $c_{91} n e g$ & $x_{1}=\frac{c_{\vartheta 1} p_{10 S}}{E_{S}}$ & $x_{2}=\frac{c_{91}{ }_{11} n e g}{E_{S}}$ & $\frac{x_{1}}{\bar{x}}$ & $\frac{x_{2}}{\bar{x}}$ & \\
\hline & {$\left[\mathrm{N} / \mathrm{mm}^{2}\right]$} & {$[\mathrm{kNm} / \mathrm{m}]$} & {$[\mathrm{kNm} / \mathrm{m}]$} & {$\left[1000 \mathrm{~mm}^{2}\right]$} & {$\left[1000 \mathrm{~mm}^{2}\right]$} & {$[-]$} & {$[-]$} & \\
\hline \multirow{4}{*}{ D1/MW } & \multirow{3}{*}{6,9} & 5,17 & 5,43 & 0,75 & 0,79 & 0,98 & 1,03 & \\
\hline & & 5,00 & 5,21 & 0,72 & 0,75 & 0,95 & 0,99 & \\
\hline & & 5,30 & 5,56 & 0,77 & 0,81 & 1,00 & 1,05 & \\
\hline & & \multicolumn{2}{|c|}{ Mittelwert $\bar{x}$} & \multicolumn{2}{|c|}{$\underline{0,77}$} & \multicolumn{2}{|c|}{$\underline{5 \% \text {-Fraktilwert }}$} & $\underline{0,69 \times \mathrm{E}_{\underline{s}}}$ \\
\hline \multirow{7}{*}{ W2/MW } & \multirow{3}{*}{8,9} & 4,50 & 4,40 & 0,51 & 0,49 & 0,96 & 0,94 & \\
\hline & & 4,55 & 5,02 & 0,51 & 0,56 & 0,97 & 1,07 & \\
\hline & & 4,66 & 5,00 & 0,52 & 0,56 & 0,99 & 1,07 & \\
\hline & & \multicolumn{2}{|c|}{ Mittelwert $\bar{x}$} & \multicolumn{2}{|c|}{$\underline{0,53}$} & \multicolumn{2}{|c|}{$\underline{5 \% \text {-Fraktilwert }}$} & $\underline{0,48 \times \mathrm{E}_{\underline{s}}}$ \\
\hline & & & & \multicolumn{2}{|c|}{ Anzahl Werte $n=$} & \multicolumn{2}{|c|}{12} & \\
\hline & & & & \multicolumn{2}{|c|}{ Variationskoeffizient s= } & \multicolumn{2}{|c|}{0,0455} & \\
\hline & & & & \multicolumn{2}{|c|}{$\mathrm{k}_{\mathrm{s}}=$} & \multicolumn{2}{|c|}{2,05} & \\
\hline
\end{tabular}

Tabelle A5.3: statistische Auswertung der Werte $c_{91}$ der Elemente mit Mineralfaser-Kernschicht

\begin{tabular}{|c|c|c|c|c|c|c|c|c|c|}
\hline \multirow[t]{2}{*}{$\begin{array}{l}\text { Versuchs- } \\
\text { bezeich- } \\
\text { nung }\end{array}$} & $\begin{array}{c}E_{s} \\
\text { (Mittel- } \\
\text { wert) }\end{array}$ & $t_{\text {Koben }}$ & $c_{92}$ pos & $c_{92} n e g$ & $x_{1}=\frac{c_{92}-p o s}{E_{S} \cdot t_{K}}$ & $x_{2}=\frac{c_{\vartheta 2}-n e g}{E_{S} \cdot t_{K}}$ & $\frac{x_{1}}{\bar{x}}$ & $\frac{x_{2}}{\bar{x}}$ & \\
\hline & {$\left[\mathrm{N} / \mathrm{mm}^{2}\right]$} & {$[\mathrm{mm}]$} & {$[\mathrm{kNm} / \mathrm{m}]$} & {$[\mathrm{kNm} / \mathrm{m}]$} & {$\left[1000 \mathrm{~mm}^{2}\right]$} & {$\left[1000 \mathrm{~mm}^{2}\right]$} & {$[-]$} & {$[-]$} & \\
\hline \multirow{7}{*}{ D1/MW } & \multirow{3}{*}{6,9} & \multirow{3}{*}{0,71} & 1,06 & 1,03 & 0,22 & 0,21 & 0,92 & 0,89 & \\
\hline & & & 1,25 & 1,32 & 0,26 & 0,27 & 1,09 & 1,15 & \\
\hline & & & 1,09 & 1,16 & 0,22 & 0,24 & 0,95 & 1,01 & \\
\hline & & & \multicolumn{2}{|c|}{ Mittelwert $\bar{x}$} & \multicolumn{2}{|c|}{$\underline{0,24}$} & \multicolumn{2}{|c|}{ 5\%-Fraktilwert } & $\underline{0,18 \times E_{\underline{s}}} \underline{x t}$ \\
\hline & & & & & \multicolumn{2}{|c|}{ Anzahl Werte $n=$} & \multicolumn{2}{|c|}{6} & \\
\hline & & & & & \multicolumn{2}{|c|}{ Variationskoeffizient s= } & \multicolumn{2}{|c|}{0,0991} & \\
\hline & & & & & \multicolumn{2}{|c|}{$k_{s}=$} & \multicolumn{2}{|c|}{2,34} & \\
\hline
\end{tabular}

\begin{tabular}{|c|c|c|c|c|c|c|c|c|c|}
\hline \multirow{7}{*}{ W2/MW } & \multirow{3}{*}{8,9} & \multirow{3}{*}{0,56} & 1,32 & 1,24 & 0,26 & 0,25 & 0,87 & 0,82 & \\
\hline & & & 1,42 & 1,88 & 0,28 & 0,38 & 0,94 & 1,24 & \\
\hline & & & 1,32 & 1,93 & 0,26 & 0,39 & 0,87 & 1,27 & \\
\hline & & & \multicolumn{2}{|c|}{ Mittelwert $\bar{x}$} & \multicolumn{2}{|c|}{$\underline{0,30}$} & \multicolumn{2}{|c|}{$\underline{5 \% \text {-Fraktilwert }}$} & $\underline{0,16 \times E_{\underline{s}} \times t}$ \\
\hline & & & & & \multicolumn{2}{|c|}{ Anzahl Werte $\mathrm{n}=$} & \multicolumn{2}{|c|}{6} & \\
\hline & & & & & \multicolumn{2}{|c|}{ Variationskoeffizient s= } & \multicolumn{2}{|c|}{0,2011} & \\
\hline & & & & & \multicolumn{2}{|c|}{$\mathrm{k}_{\mathrm{s}}=$} & \multicolumn{2}{|c|}{2,34} & \\
\hline
\end{tabular}

Tabelle A5.4: statistische Auswertung der Werte $c_{92}$ der Elemente mit Mineralfaser-Kernschicht 